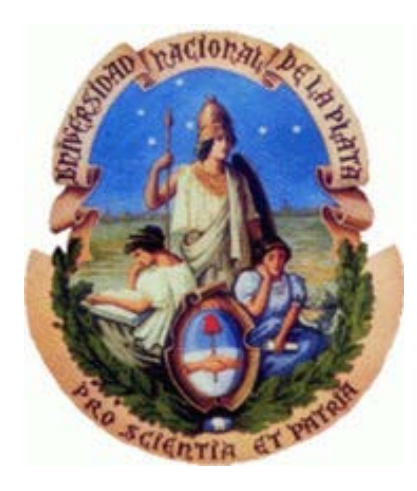

Universidad Nacional de La Plata

Facultad de Ciencias Exactas

Departamento de Física

Tesis Doctoral

\title{
Estudio de rayos cósmicos ultraenergéticos en el Observatorio \\ Pierre Auger: Nuevo modelo de profundidad atmosférica y su influencia en observables.
}

\author{
Lic. Juan Cruz Moreno \\ Director: Dr. Sergio Sciutto
}

Abril de 2012 

a Ceci,

banca completa en este lío. 



\section{Índice general}

Índice general $\quad 5$

$\begin{array}{lr}\text { Introducción } & 9\end{array}$

1. Breve reseña temática e histórica 13

2. Física de Rayos Cósmicos $\quad 19$

2.1. Origen de los Rayos Cósmicos . . . . . . . . . . . . . . . . . 19

2.2. Propagación de los Rayos Cósmicos . . . . . . . . . . . 22

2.2.1. ¿Isotropía o Anisotropía? . . . . . . . . . . . . . 30

3. Los Rayos Cósmicos en la Atmósfera 31

3.1. Evolución de las cascadas . . . . . . . . . . . . . . . 32

3.2. Modelización de la cascada . . . . . . . . . . . . . . . . . 37

3.2.1. El Modelo de Heitler . . . . . . . . . . . . . . . . 37

3.2.2. El Teorema del Elongation Rate . . . . . . . . . . . . . 39

3.3. Detectores de Superficie: Determinación de los parámetros de

la lluvia . . . . . . . . . . . . . . . . . . . . 42

3.3.1. El eje y la dirección de la lluvia . . . . . . . . . . . . 42

3.3.2. La Energía de la partícula primaria . . . . . . . . . . 47

3.3.3. La función de distribución lateral . . . . . . . . . . . 48

3.4. Detector de Fluorescencia: Determinación de los parámetros

de la lluvia . . . . . . . . . . . . . . . . . . . . . . . . . 49

3.4.1. Geometría de la lluvia . . . . . . . . . . . 50

3.4.2. Determinación de la Energía del primario . . . . . . . 52

4. El Observatorio Pierre Auger $\quad 57$

4.1. Características generales del Observatorio Auger . . . . . . . . 57

4.2. El arreglo de detectores de superficie . . . . . . . . . . . . . 59

4.2.1. Calibración de los tanques Cherenkov . . . . . . . . . 60

4.2.2. Trigger y Adquisición de Datos . . . . . . . . . . . 61 
4.2.3. Algunos comentarios adicionales sobre los tanques SD . 66

4.3. Detectores de fluorescencia . . . . . . . . . . . . . . . . . 69

4.3.1. Trigger ................... 71

4.3.2. Calibración de las cámaras . . . . . . . . . . . 73

4.4. Otros detectores en desarrollo . . . . . . . . . . . . . . 75

4.5. El monitoreo atmosférico . . . . . . . . . . . . . . . . 77

4.5.1. BLS : Estación de lanzamientos de globos . . . . . . 79

4.5.2. Las estaciones meteorológicas . . . . . . . . . . 80

4.5.3. LIDAR . . . . . . . . . . . . . . . . . 81

4.5.4. Central y eXtreme Laser Facilities (CLF/XLF) . . . . 83

4.5.5. Cámaras infrarrojas para nubosidad . . . . . . . . . 84

4.5.6. Otros sistemas de monitoreo atmosférico . . . . . . . . 85

4.6. Adquisición, procesamiento y análisis de datos . . . . . . . . . 85

5. Los perfiles de profundidad atmosférica $\quad 87$

5.1. Breve descripción de la Atmósfera. . . . . . . . . . . . . . . . . 87

5.2. Los Modelos Clásicos . . . . . . . . . . . . . . . . . . 90

5.2.1. Modelo Isotérmico . . . . . . . . . . . . 90

5.2.2. Modelo de Linsley . . . . . . . . . . . . . . . . 93

5.3. Modelos recientes . . . . . . . . . . . . . . 96

5.3.1. El Modelo Mensual (Malargüe Monthly Model) . . . . . 96

5.3.2. El Nuevo Modelo Mensual (New Malargüe

Monthly Model . . . . . . . . . . . . . . . . . 99

6. El modelo GAMMA 103

6.1. Ideas motivadoras . . . . . . . . . . . . . . . . . . 103

6.2. La base de datos . . . . . . . . . . . . . . . . . . . . 104

6.3. La función de ajuste . . . . . . . . . . . . . . . . . . 110

6.4. Resultados de los ajustes . . . . . . . . . . . . . . . . 111

6.5. Verificando el Modelo GAMMA . . . . . . . . . . . . . . . . 115

6.5.1. Estudio de Residuos . . . . . . . . . . . . . . . 115

6.5.2. Otra comparación con Linsley . . . . . . . . . . . . . . 119

6.5.3. El uso en el Observatorio Auger: más sobre las predicciones del Modelo GAMMA y del Modelo NMMM . . . 121

6.5.4. Chequeo de consistencia . . . . . . . . . . . . . . 123

6.6. Rango de validez de GAMMA . . . . . . . . . . . . 125

7. Estudios sobre el Modelo GAMMA 131

7.1. Modelo de capas no isotérmico . . . . . . . . . . . . . . . . 131

7.1.1. Construcción de la función . . . . . . . . . . . . . . 132

7.1.2. El ajuste . . . . . . . . . . . . . . 134 
7.1.3. Discusión y Resultados . . . . . . . . . . . . . . 135

7.2. Parametrización polinómica de $\operatorname{los} c_{l} \ldots \ldots$. . . . . . . 141

7.3. Modelo aumentando grados de libertad . . . . . . . . . . . 143

7.4. Estudio en otros sitios . . . . . . . . . . . . . . . . 148

7.4.1. Síntesis del Procedimiento . . . . . . . . . . . 150

7.4.2. Dodge City . . . . . . . . . . . . . . 150

7.4.3. Base Halley . . . . . . . . . . . . . . . . . . . . 157

7.4.4. Comentario final acerca de GAMMA en otros sitios . . 158

8. GAMMA y los observables 165

8.1. Distribución lateral al nivel del suelo . . . . . . . . . . . . 165

8.1.1. Más sobre la función de distribución lateral (LDF) . . . 165

8.1.2. Análisis con GAMMA . . . . . . . . . . . . 167

8.2. Desarrollo longitudinal . . . . . . . . . . . . . . . . . . . 181

8.2.1. Modelos atmosféricos y Desarrollo Longitudinal . . . . 181

8.2.2. GAMMA y $X_{\max } \ldots \ldots \ldots . \ldots . \ldots 182$

8.2.3. GAMMA y el número de partículas cargadas . . . . . . 190

9. Resumen, Conclusiones y Perspectivas 199

$\begin{array}{ll}\text { Appendices } & 204\end{array}$

A. Obtención de los parámetros de $X_{G A M M A} \quad 207$

B. Geometría de las lluvias para el cálculo de la LDF 209

C. Parámetros $\beta \quad 213$

$\begin{array}{ll}\text { Bibliografía } & 215\end{array}$ 



\section{Introducción}

Los rayos cósmicos son partículas subatómicas con un amplio espectro de energía que alcanzan la Tierra provenientes de diferentes sitios del espacio. En los últimos años se ha ampliado la posibilidad de registrar eventos producidos por rayos cósmicos con energías mayores a $10^{18} \mathrm{eV}$. Su estudio permite establecer propiedades características de la propagación en el medio intergaláctico, identificar los cuerpos celestes capaces de permitirles adquirir esa energía, profundizar sobre modelos teóricos acerca de los procesos ligados a los mecanismos de aceleración y dilucidar la naturaleza de las partículas primarias.

Sobre esos objetivos se construyó el Observatorio Pierre Auger, el mayor experimento actual para la detección de rayos cósmicos y en el que trabajan desde 1998 una colaboración de 450 científicos de 17 países, localizado cerca de la ciudad de Malargüe en Mendoza, Argentina. El experimento detecta las partículas secundarias que conforman la llamada cascada o lluvia, producida a partir del ingreso de los rayos cósmicos a la atmósfera. La característica principal de la detección del Observatorio Pierre Auger es que utiliza un arreglo de detectores híbridos compuesto por dos técnicas complementarias de observación de las lluvias: estudia el desarrollo de la cascada en la atmósfera mediante un arreglo de detectores de fluorescencia y analiza mediante detectores de superficie aquellas partículas que alcanzan el nivel del suelo.

La presente Tesis de Doctorado está enmarcada en el estudio de rayos cósmicos dentro del Observatorio Pierre Auger. Su temática se vincula a la necesidad, propia de un experimento de estudio de rayos cósmicas, de conocer las características de la atmósfera en el instante en que sucede un evento. En particular se ha estudiado la profundidad atmósferica, parámetro de suma importancia vinculado a la cantidad de materia atravesada por las partículas en su trayecto por la atmósfera. En base a una idea original hemos construido un modelo que permite reproducir los perfiles de profundidad atmósferica en cualquier instante de tiempo, a partir de un parámetro de fácil y de permanente acceso como lo es el valor de la temperatura en el 
suelo. Este modelo que hemos denominado GAMMA, fue desarrollado en base a mediciones realizadas en el sitio del Observatorio Auger. Sin embargo, y así queda reflejada en estudios indicados en el presente trabajo, su idea central es aplicable a otros sitios. El desarrollo del modelo GAMMA incluyó también una serie de análisis referidos a optimizar sus predicciones y su rango de validez.

Los resultados alcanzados con el modelo GAMMA son muy satisfactorios. Se consigue reproducir los perfiles de profundidad atmosférica con un muy bajo nivel de incerteza. Asimismo, ha salido exitoso en la comparación con otros modelos previamente aplicados en estudios del Observatorio Auger, reflejando incertezas similares o incluso menores.

Los estudios vinculados a la influencia del perfil atmósferico, éste modelizado mediante GAMMA, en el desarrollo longitudinal y lateral de las cascadas también han producidos resultados relevantes. Por ejemplo hemos identificado una sobreestimación al utilizar los modelos atmósfericos típicos en la determinación de los valores del parámetro $S_{1000}$ vinculado a la energía en el estudio con detectores de superficie; y la confirmación de la posición del máximo desarrollo de la cascada $X_{\max }$ como parámetro válido en la determinación de la composición primaria.

Las tareas de investigacion vinculadas a esta Tesis fueron realizados en el Instituto de Física de La Plata (CONICET-UNLP), la Universitá Tor Vergata de Roma, y el Observatorio Pierre Auger de Malargüe.

El presente documento ha sido estructurado de la siguiente manera. En el capítulo 1 se decriben los comienzos y las motivaciones en el área de investigación de rayos cósmicos. El capítulo 2 presenta un breve panorama del origen y propagación de los rayos cósmicos en el medio galáctico e intergaláctico, así como de los modelos cosmológicos actuales. En el capítulo 3 se desarrolla el tema de la producción de las cascadas originadas por la interacción de las partículas primarias con los núcleos de los átomos de la atmósfera. Esta primera parte sobre rayos cósmicos se completa en el capítulo 4 con la descripción del Observatorio Pierre Auger, con especial hincapié en su técnica híbrida de detección.

La Tesis continúa en el capítulo 5 con una descripción de la atmósfera y los principales modelos para los perfiles de profundidad atmosférica. En el capítulo 6 se encuentra la descripción del modelo GAMMA, su desarrollo y la determinación de sus predicciones. Dentro del capítulo 7 se reflejan diferentes estudios realizados acerca del modelo GAMMA, vinculadas a modificar las funciones de parametrización y la aplicación del modelo en otros sitios. En el capítulo 8 se discute la influencia del modelo GAMMA en parámetros observables de las lluvias, vinculados a su desarrollo longitudinal y al desarrollo lateral al nivel del suelo. El desarrollo de esta Tesis concluye en el capítulo 9, 
en donde se resumen los conceptos originales de la misma, se presentan las conclusiones alcanzadas y se incluyen asimismo breves reflexiones sobre las perspectivas a futuro. Por último se encuentran tres apéndices vinculados a temas puntuales mencionados en los capítulos previos. 


\section{Capítulo 1}

\section{Breve reseña temática e histórica}

Desde las primeras civilizaciones el hombre ha sentido una enorme curiosidad por el espacio exterior. Siempre intentó comprender su conformación, su disposición, su funcionamiento, sus dimensiones. El siglo XX no ha sido la excepción. El descubrir que radiación de un amplio espectro de energía mas allá del rango visible por el ojo humano estaba continuamente llegando a la superficie terrestre, abrió una nueva puerta en el conocimiento de la naturaleza. Incluso hoy, más de 90 anõs después de su descubrimiento algunas incógnitas siguen desvelando a la comunidad científica.

Los números asociados a esta radiación son fascinantes. Se han detectado eventos asociados a partículas relativistas que ingresan a la atmósfera terrestre con energías superiores a $10^{20} \mathrm{eV}$. Tal energía cinética es equivalente a la de una pelota de tenis moviéndose aproximadamente a $100 \mathrm{~km} / \mathrm{h}$ [1], pero concentrada en una partícula cuyo volumen es aproximadamente $10^{35}$ veces menor! Sin embargo el estudio de tales partículas no es sencillo: más allá de los mecanismos de detección, la probabilidad de que una de estas partículas impacte sobre la superficie terrestre es aproximadamente de una partícula por año cada $100 \mathrm{~km}^{2}$ de superficie terrestre. Pero debemos tener en cuenta que estos son solo los mayores valores de energía de un espectro que comienza en $10^{9} \mathrm{eV}$ y se extiende hasta más allá de $10^{20} \mathrm{eV}$, y cuyo flujo de partículas resulta muy abundante a bajas energías y decrece sustancialmente conforme aumenta la energía (Figura 1.1).

Lo antedicho establece un punto que despierta el interés científico y que puede resumirse en una afirmación concluyente: los rayos cósmicos ultra energéticos son las partículas de mayor energía en la naturaleza en proporción a su masa.

Veamos un poco la génesis de los estudios en rayos cósmicos. Victor Hess 


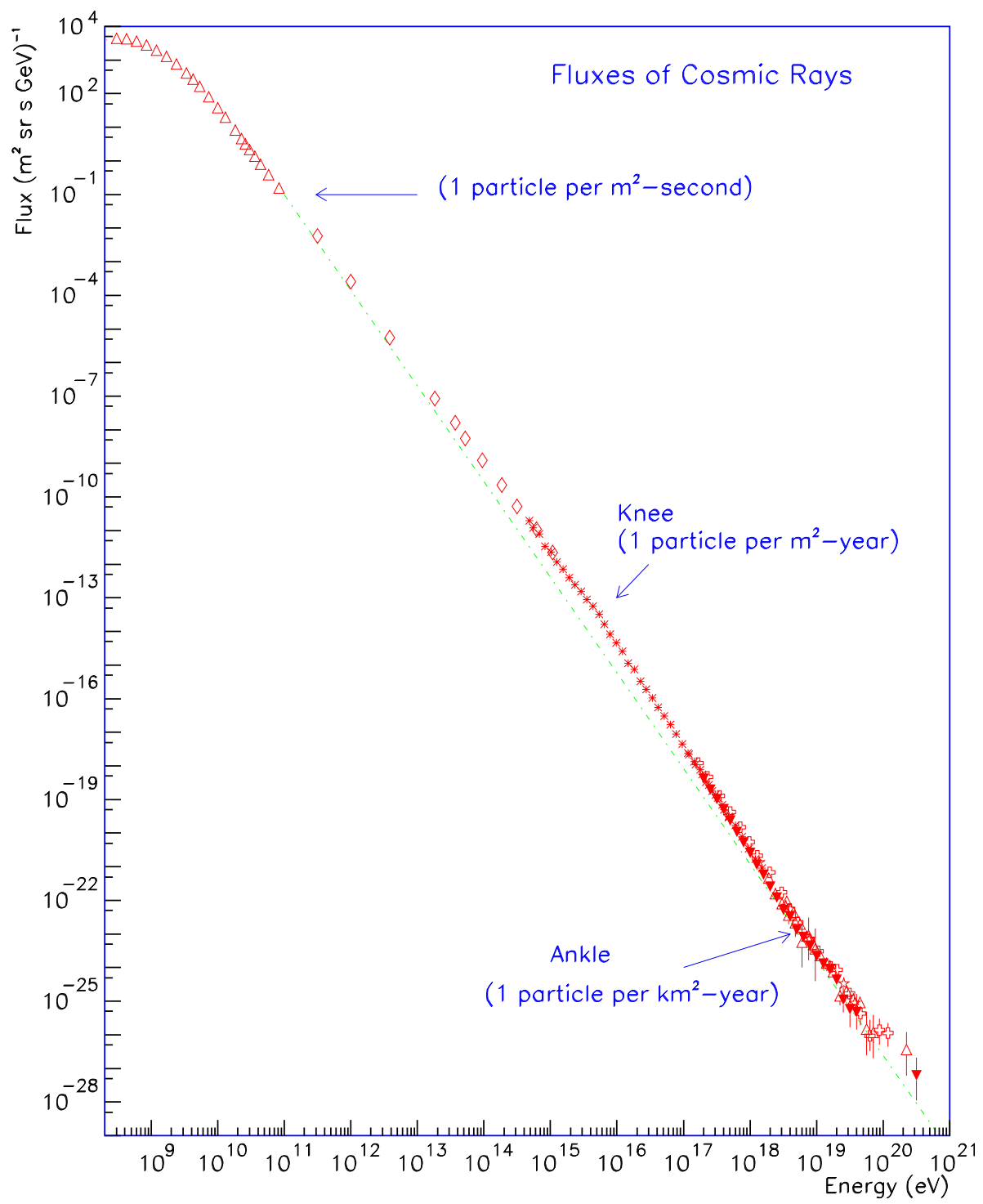

Figura 1.1: Espectro observado de rayos cósmicos en el rango de $10^{11}$ a $10^{20}$ eV. Se observan los cambios de pendiente denominados "codos" (knee) y "tobillos" (ankle) 
en 1912 fue el primero en determinar la existencia de radiación cósmica a través de una serie de experimentos con globos aerostáticos con una cámara de ionización que dejaba subir hasta $5000 \mathrm{~m}$ de altura para medir la radiación de fondo, descubriendo que ésta aumentaba con la altura, lo que planteaba la presencia de radiación externa a la tierra. Millikan en 1925 impone a esta radiación el nombre de rayos cósmicos. Estas experiencias habían permitido detectar partículas primarias con energía de hasta $10^{14} \mathrm{eV}$.

Luego Auger y sus colaboradores ([2],[3]) reportan las primeras observaciones de las propiedades de las lluvias cósmicas. El fenómeno descubierto por Auger había sido sugerido ya por Rossi (1934) que de sus observaciones en Eritrea decía: "He visto que de tiempo en tiempo arriban a mi equipamiento grupos muy extensos de partículas que producen coincidencias entre contadores, incluso situados a grandes distancia entre ellos".

Este descubrimiento de lluvias cósmicas extendidas que involucró a Auger y su grupo, provino de las mejoras técnicas en la resolución de tiempo de coincidencias que fueron capaces de medir descargas en dos contadores separados por extensas distancias. Estos contadores, del tipo Geiger-Müller, mostraban tiempos de arribos de partículas correlacionados, cuando provenían en realidad de puntos muy distantes entre sí. El meticuloso trabajo de Auger con arreglos de varios de esos contadores posicionados en montañas de distintas alturas, le permitió concluir que esas cascadas eran iniciadas por rayos cósmicos que ingresaban en la atmósfera, con una energía del primario estimada en alrededor de $10^{15} \mathrm{eV}$ mediante la recientemente desarrollada teoría de cascadas. Lo que resultó evidente, y sorprendió a la comunidad científica, es que Auger había detectado partículas con energía de cinco órdenes de magnitud mayor que las reportadas por otras experiencias hasta el momento.

Estos resultados fueron el punto de partida para ahondar en la detección de partículas de altas energías mediante el uso de detectores situados sobre el suelo. Por tal motivo este tipo de detectores comenzaron a ser llamados "detectores de superficie" (SD). Con el objeto de profundizar en la precisión de los estudios, comenzaron a establecerse conjuntos de detectores tipo contadores Geiger-Müller (denominados "arreglos de detectores") abarcando cada vez superficies más extensas. Pese a que este tipo de arreglos contribuían a ir conociendo y entendiendo las estructuras de las lluvias (detectándose eventos con energías de hasta $10^{17} \mathrm{eV}$ ), comenzaba a hacerse evidente la baja eficacia de los contadores Geiger-Müller para determinar el eje principal de desarrollo de las lluvias.

Este inconveniente fue solucionado por un grupo del Instituto Técnico de Massachussets (MIT) dirigido por Bassi, quienes en 1953 desarrollaron una técnica de reconstrucción de la dirección de llegada a partir de mediciones de tiempo de arribo de las partículas con contadores de centelleo separados por 
unas pocas decenas de metros. El grupo del MIT también desarrolló técnicas para usar la densidad de partículas observadas con arreglos de contadores de centelleo para localizar la región de mayor densidad de partículas. Entre 1954 y 1957 un arreglo de 15 contadores, cada uno de $0.9 \mathrm{~m}^{2}$ estuvo operando en la Estación Harvard Agassiz [4]. Los datos de este arreglo permitieron explorar el espectro de energía desde $3 \times 10^{15} \mathrm{eV}$ a $10^{18} \mathrm{eV}$.

El arreglo de Harvard Agassiz sirvió como prototipo para diseñar los grandes arreglos de altura realizados luego en Chacaltaya, Bolivia [5] y Volcano Ranch en Nuevo México, Estados Unidos. Este último es el arreglo que le permitió a Linsley observar el primer evento con energía estimada por encima de $10^{20} \mathrm{eV}$.

Como consecuencia de que el flujo de partículas primarias disminuye notoriamente para las partículas más energéticas, el explorar esa zona del espectro conllevó la necesidad de ampliar la superficie de los arreglos a fin de generar una estadística razonable de eventos en un tiempo acorde. Debido al número de detectores involucrado en cubrir este tipo de arreglos mas extensos, y los costos económicos por detector, los detectores de centelleo fueron cediendo terreno al uso de detectores de efecto Cherenkov. Citemos como históricos experimentos con grandes arreglos de detectores tipo Cherenkov a Haverah Park (Gran Bretaña), Yakutsk (Rusia), AGASA (Japón); y como experimentos con centelleadores a Volcano Ranch (Estados Unidos).

Los grandes arreglos de detectores de superficie no constituyen el único tipo de instrumento utilizado para detectar rayos cósmicos ultraenergéticos. Suga [7] y Chudakov [8] plantearon la posibilidad de observar la emisin de fluorescencia producidas por las partculas en su paso a travs de la atmsfera y realizar una medicin calorimtrica de la energa all depositada. La idea tuvo un avance posterior con el empuje de Greisen (1965) quien construyó un prototipo en Cornell, Estados Unidos. Los primeros capaces de utilizarlo en la detección de luz de fluorescencia, producida por la interacción de partículas cargadas con los átomos de Nitrógeno propios del aire, que correspondía a eventos producidos por partículas primarias de energía $10^{19} \mathrm{eV}$, fueron Tanahashi y sus colaboradores sobre el Monte Dodaira cercano a Tokio [9]. Cornell y Tokio no eran los sitios ideales para continuar con las experiencias debido a sus condiciones climáticas, con lo cual Keuffel y su grupo continuaron la investigación en Volcano Ranch. En 1976 realizaron la primera detección de emisión fluorescente proveniente de una lluvia cósmica en coincidencia con el arreglo de detectores de superficie [10]. Este suceso dió pie al desarrollo de detectores tipo Fly's Eye, consistentes en conjuntos de fotomultiplicadores agrupados de forma tal de componer una estructura que lleva a recordar a los ojos de las moscas. Cada uno de los fotomultiplicadores observa en una direccíon determinada y la composición del evento de fluores- 
cencia se logra por conjunción de las señales. Uno de los detectores emblema tipo Fly's Eye de alta resolución, se encuentra en el experimento HiRes que está localizado en Utah, Estados Unidos.

A comienzos de la década de 1990 un grupo de investigadores de la comunidad de rayos cósmicos, encabezados por James Cronin (Premio Nobel de Física 1980) y Alan Watson, comenzó a idear la posibilidad de combinar ambas técnicas de detección en un experimento de rayos cósmicos de ultra-alta energía. El resultado es el Observatorio Pierre Auger, funcionando desde 1998 y que combina los arreglos de superficie y de fluorescencia en el llamado "modo híbrido" de detección. Mas allá de que las características de este Observatorio serán desarrolladas en las próximas páginas, evidenciemos su magnitud en el contexto de los experimentos de rayos cósmicos previamente citados. La ya mencionada innovación en el original modo de detección híbrido es acompañada por las enormes dimensiones involucradas: 1600 detectores de superficie tipo Cherenkov que cubren $3000 \mathrm{~km}^{2}$ componen el arreglo de superficie, y 42240 fotomultiplicadores distribuidos en 24 cámaras en 4 telescopios componen el detector de fluorescencia.

En definitiva buscamos que quede claro un punto: mucho se ha recorrido desde las primeras mediciones de Hess, ahora es el turno de otros experimentos referentes, y el Observatorio Pierre Auger es el presente en el campo de los experimentos de rayos cósmicos. 


\section{Capítulo 2}

\section{Física de Rayos Cósmicos}

Como fue mencionado anteriormente, el eje de la investigación en el Observatorio Pierre Auger, así como en esta tesis; son los rayos cósmicos de energía superior a $10^{18} \mathrm{eV}$. Las principales inquietudes a dilucidar sobre estos rayos cósmicos ultraenergéticos están relacionados con comprender el origen, la propagación y la naturaleza de éstas partículas con tan alta energía. Si pensamos que las partículas con mayor energía detectadas (presumiblemente protones o núcleos atómicos con energía del orden $10^{20} \mathrm{eV}$ ) poseen una energía mayor en varios órdenes de magnitud que la obtenida a través del más poderoso acelerador de partículas construido por el hombre; una serie de cuestiones surgen inmediatamente.¿Qué objetos astrofísicos las producen? ¿Cómo es su propagación en el espacio exterior? ¿De qué tipo de partículas primarias se trata? ¿Se podrán detectar partículas con energías superiores? La respuesta a estos interrogantes es la búsqueda actual de la comunidad de rayos cósmicos. A continuación, una serie de comentarios, brevemente desarrollados, con los consensos establecidos sobre estos tópicos.

\subsection{Origen de los Rayos Cósmicos}

Una discusión completa sobre las fuentes generadores de estos rayos cósmicos ultraenergéticos supera los objetivos planteados en este trabajo por la complejidad que involucran los modelos planteados. Trabajos recientes sobre estos modelos pueden encontrarse en las referencias [11], [12], [13], [14], [15]. Comentemos las líneas generales de las actuales ideas. Primeramente establezcamos las clasificación de los modelos utilizado: los mecanismos de producción de estas partículas se clasifican en dos categorías, los modelos bottom-up y los modelos top-down.

Los modelos bottom-up sugieren que las partículas pasan de un estado de 
energía menor a otro de energía mayor mediante un proceso de aceleración, conocido como mecanismo de Fermi [16], en procesos que se sabe que suceden en objetos astrofísicos [17], [18] .

El mecanismo de la aceleración de Fermi es a menudo denominado aceleración difusiva, y se produce dentro de una región confinada en objetos astrofísicos donde la interacción permanente de algunas partículas cargadas y núcleos con otras partículas generadas por los mismos objetos les permite adquirir a los primeros altas energías que ocasionalmente les permite escapar de ese confinamiento. El campo magnético $B$ producido en estas zonas de confinamiento juega un rol crucial.

El fenómeno de la aceleración de Fermi ya se ha comprobado aunque para rangos mucho menores de energía. Uno de esos casos ha sido la observación de la heliósfera a través del satélite ISEE que determinó la aceleración de protones en el rango 10 a $100 \mathrm{keV}$ por ondas de choque del viento solar. Los datos concuerdan con los valores predichos por la teoría de la aceleración de Fermi.

Una simple estimación realizada por Hillas en 1994 sobre el modelo de la aceleración de Fermi, nos permite obtener una sencilla expresión para la máxima energía $E_{\max }$ que puede adquirir una partícula de carga Ze. La misma está dada por

$$
E_{\max } \sim \beta \cdot c \times k \cdot Z e \times B \times L
$$

donde $\mathrm{L}$ es el tamaño característico de la región de aceleración y $\beta \cdot c$ es la velocidad de las ondas de choque. El parámetro $\beta$ es del orden de 0.01 en el caso de las supernovas. Sin embargo bajo ciertas configuraciones del campo magnético presente, $\beta$ es reemplazada por un valor mucho mayor, del orden 1-3 [18], [19]. La ecuación (3.1) en definitiva establece que el radio de giro de la partícula siendo acelerada debe ser menor que las dimensiones de las regiones de confinamiento. La especulación de que estos aceleradores astrofísicos tengan una eficiencia del $100 \%$ no es acertada; así que es razonable pensar en un valor de $E_{\max }$ reducido en un factor diez respecto al predicho por la ecuación, dependiendo de los detalles del entorno de la región (Figura 2.1).

Una de las regiones del Universo donde se considera que mediante el mecanismo de Fermi podrían producirse partículas con energía mayores a $10^{18} \mathrm{eV}$ son los lóbulos de radio galaxias, en particular zonas dentro de los lóbulos, llamadas "manchas calientes".

Los modelos top-down implican el decaimiento de una partícula supermasiva dando como uno de sus productos finales a los rayos cósmicos [20]. Una de las fuentes de estas partículas exóticas podrían ser los Defectos Topológicos que serían vestigios de transiciones de fase en el universo temprano con 


\section{Hillas-plot}

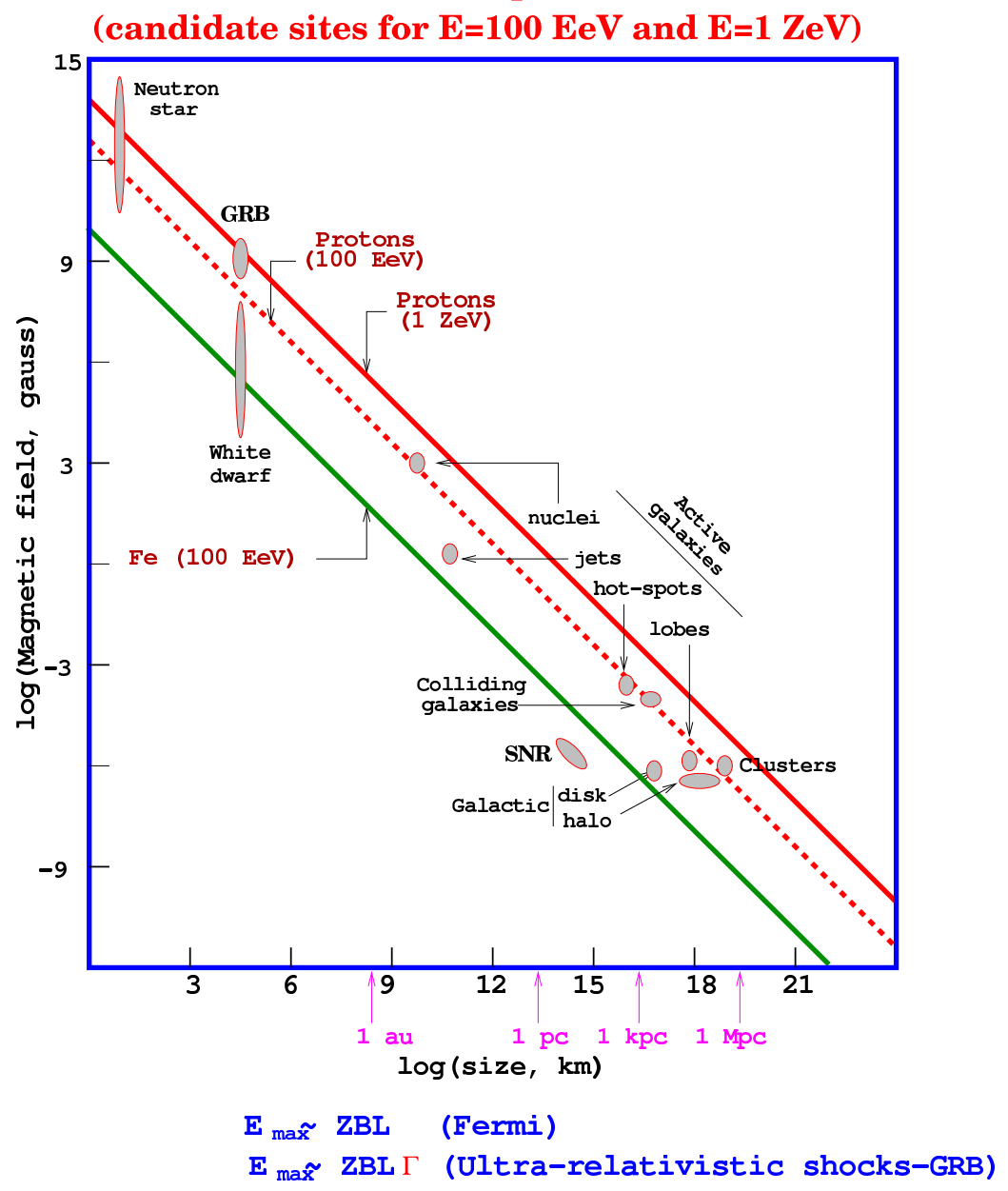

Figura 2.1: Posibles objetos astronómicos capaces de generar y acelerar partículas ultarenergétocas. Ubicación en función del tamaño e intensidad del campo magnético. Los objetos por debajo de la línea diagonal no pueden acelerar protones hasta energías de $10^{20} \mathrm{eV}$. 
rupturas espontáneas de simetría. Los defectos topológicos podrían existir al tiempo presente y estar degradándose en sus campos constituyentes. Estas partículas X supermasivas (con energías del orden de $10^{24} \mathrm{eV}$ ) decaerían dando como resultados leptones y quarks

$$
X \longrightarrow q \bar{q} l
$$

Los leptones rápidamente interactúan produciendo rayos $\gamma$ y electrones de mucha menor energía. Sin embargo los quarks inmediatamente se fragmentan en jets de hadrones, resultando aproximadamente $10^{4}-10^{5}$ partículas entre bariones y mesones.

$$
q \bar{q} \longrightarrow \pi^{ \pm}, \pi^{0}, p, n
$$

A su vez, los mesones $\pi^{ \pm}$y $\pi^{0}$ producen decaimientos

$$
\begin{gathered}
\pi^{+} \longrightarrow \mu^{+}+\nu_{\mu} \longrightarrow e^{+}+\nu_{e}+\bar{\nu}_{\mu}+\nu_{\mu} \\
\pi^{-} \longrightarrow \mu^{-}+\bar{\nu}_{\mu} \longrightarrow e^{-}+\bar{\nu}_{e}+\nu_{\mu}+\bar{\nu}_{\mu} \\
\pi^{0} \longrightarrow \gamma+\gamma
\end{gathered}
$$

Estos modelos top-down proveen rayos cósmicos dominados fundamentalmente por neutrinos, nucleones y rayos $\gamma$ que caracterizarán a estos procesos. La componente de protones comprende sólo un pequeño porcentaje del flujo total. A partir de esto es que se considera que varios de estos defectos topológicos son capaces de producir rayos cósmicos de ultra alta energía.

La teoría predice que estos mecanismos no aportan creación de núcleos pesados con alta energía sino sólo protones y partículas sin masa. Además se preveé que estos modelos contribuyan significativamente con partículas del orden de $10^{20} \mathrm{eV}$ y no aporten de manera importante al rango de energía de $10^{14}-10^{19}$.

Mediciones más precisas sobre los cuerpos celestes han hecho que actualmente los modelos bottom-up tengan más adeptos que los top-down, aunque estos últimos no se han descartado totalmente.

\subsection{Propagación de los Rayos Cósmicos}

Hay otro factor que debe tomarse en cuenta cuando se piensa en la búsqueda del origen de los rayos cósmicos: su propagación en el medio galáctico y extragaláctico y la posibilidad de la interacción con el fondo de radiación de microondas. 


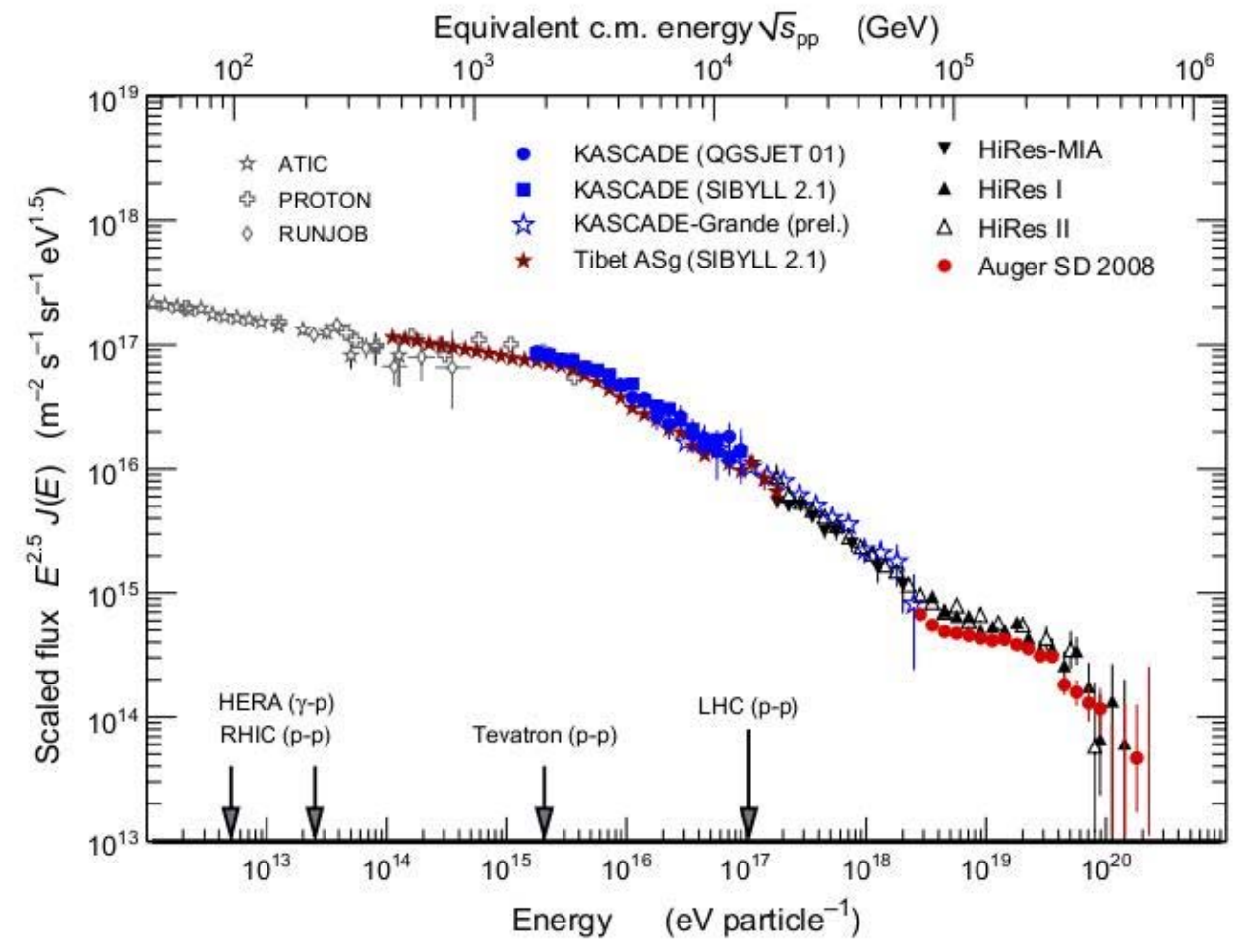

Figura 2.2: Espectro de energía de rayos cósmicos obtenido a partir de recientes mediciones [57]. Nótese los cambio de pendiente alrededor de $10^{15.5}$ eV, conocido como "rodilla"; y cercano a $10^{18.5}$ eV, conocido como "tobillo". 
La observación del espectro de energía de las partículas primarias (Figura 2.2) nos revela ciertos detalles de la propagación entre $1 \mathrm{GeV}$ y $100 \mathrm{EeV}$. La forma del espectro sugiere una ley de potencias con pequeños cambios en la pendiente. Estas regiones donde ocurren los cambios se conocen como "rodilla" (knee) para el cambio alrededor de $10^{15.5} \mathrm{eV}$ y "tobillo" (ankle) para el cambio aproximadamente en $10^{18.5} \mathrm{eV}$. Debemos considerar también que los flujos por encima de $10^{9} \mathrm{eV}, 10^{16} \mathrm{eV}$ y $10^{19} \mathrm{eV}$ son $\sim 1 / \mathrm{m}^{2} \mathrm{~s}, \sim 1 / \mathrm{m}^{2}$ año, $\sim 1 / \mathrm{km}^{2}$ año respectivamente.

Del espectro de energía se desprende un comportamiento natural, para muchos sorprendente, que relaciona el flujo con la energía. Esta relación es la que antes mencionamos que se suele aproximar por una ley de potencias, dada por $J=k E^{-\gamma}$. Los últimos resultados del Observatorio Auger [58] (Figura $2.3)$, determinan la posición del tobillo en $\log _{10}\left(E_{\text {ankle }} / \mathrm{eV}\right)=18,61 \pm 0,01 \mathrm{y}$ el coeficiente de la función resulta $\gamma=3,26 \pm 0,04$ para la región comprendida entre la rodilla y el tobillo. Más allá del tobillo, el espectro sigue una ley de potencias con exponente $\gamma=2,59 \pm 0,02$. Este trabajo además ajusta el flujo suavizando la ley de potencias, la expresión propuesta es

$$
J\left(E ; E>E_{\text {ankle }}\right) \propto \frac{E^{-\gamma}}{1+\exp \left(\frac{\log _{10} E-\log _{10} E_{1 / 2}}{\log _{10} W_{c}}\right)}
$$

donde $\mathrm{E}_{1 / 2}$ es la energía a la cual el flujo ha disminuido a la mitad del valor de la extrapolación de la ley de potencias y $W_{c}$ es un coeficiente que parametriza el ancho de la región de transición. Con esta función, el coeficiente alcanzado en el ajuste para la región posterior al tobillo es $\gamma=2,55 \pm 0,04$.

Estos cambios de pendientes han sido interesantes objetos de estudio. En particular, la zona de la rodilla se considera un escenario de aceleración. Se asocia al cambio de pendiente a la altura de la rodilla con una transición entre diferentes clases de rayos cósmicos galácticos, o con alguna característica de la propagación de los rayos cósmicos en la galaxia [25].

Uno de los modelos sugiere que la región del espectro anterior al tobillo, representa una transición entre los rayos cósmicos con un origen galáctico y aquellos que tienen un origen extragaláctico [61], [62]. En este modelo la componente extragaláctica se piensa como compuesta exclusivamente por protones. El tobillo representaría la energía a la cual las componentes contibuyen igualmente al flujo total.

Otro modelo [63], [64] establece que la cola del flujo tiene una composición mixta y el tobillo es entonces la transición del origen de los rayos cósmicos (Figura 2.4(izq)). Los núcleos con energías mayores a $10^{19} \mathrm{eV}$ son desintegrados mediante se propagan.

Un tercer modelo [59], [65], conocido como dip model, considera que la 


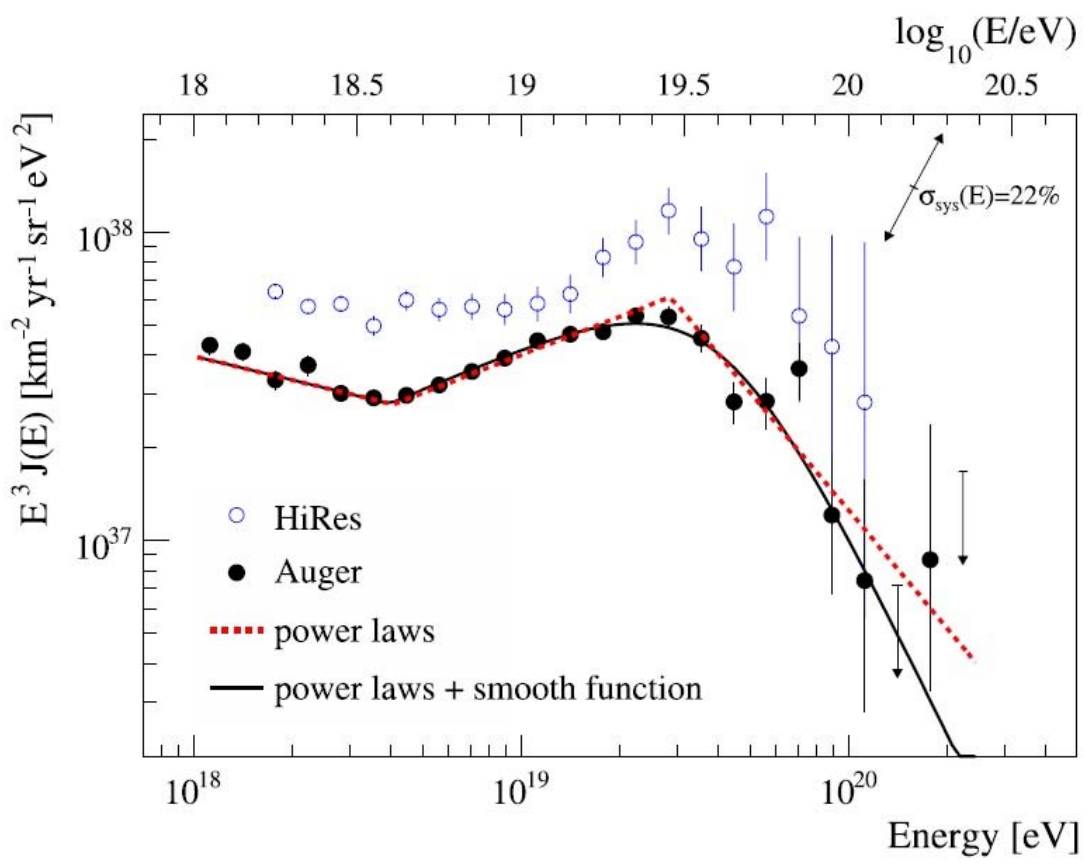

Figura 2.3: Espectro de energía de rayos cósmicos en la region $10^{18}-10^{20} \mathrm{eV}$ [58]. Sobre los datos se encuentra ajustada una función continua a trozos con forma de ley de potencias $J=k E^{-\gamma}$ (en rojo), y otra suavizada de acuerdo a la ecuación (2.2) (en negro). 

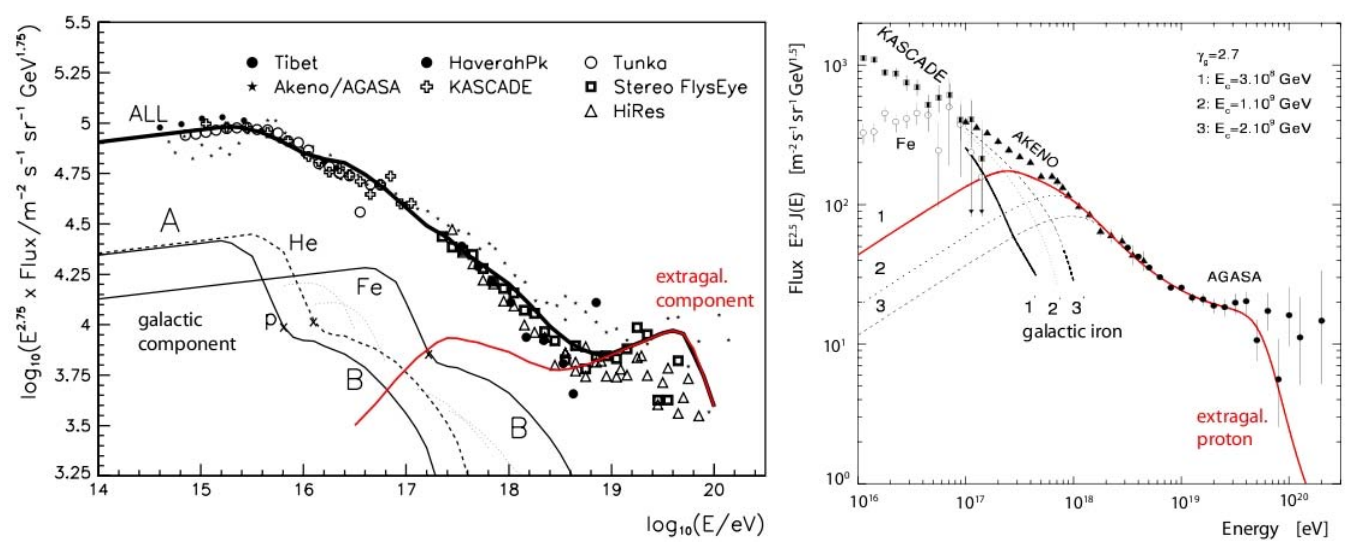

Figura 2.4: Espectro mostrando el modelo de composición mixta (izq). El Dip model con los protones dominando la componente extragaláctica a bajas energías (der).

componente extragaláctica compuesta mayormente por protones comienza a dominar a energías un poco menores (Figura 2.4(der)). En la región del tobillo la componente galáctica ya es prácticamente nula. El cambio en el índice espectral es entonces un efecto de propagación: los protones atraviesan el fondo cósmico de microondas perdiendo energía via producción de $\mathrm{e}^{+} \mathrm{y} \mathrm{e}^{-}$ y esto causa una supresión de flujo a grandes energías y una leve acumulación a energías levemente inferior.

Si analizamos primero el caso de la propagación dentro de nuestra galaxia, los protones ultraenergéticos no sufren pérdidas de energía significantes en su viaje, pero sí sufren deflexiones por su interacción con el campo magnético galáctico. Si todos los rayos cósmicos provinieran de fuentes dentro de la galaxia, deberían exhibir una tendencia a viajar por el plano del disco de la galaxia de acuerdo al tipo de interacción magnética. Hasta el presente no hay evidencia estadística de esto, que implique una dirección preferencial en la llegada de rayos cósmicos lo cual hace razonable suponer que las partículas con $E>10^{19} \mathrm{eV}$ tiene origen extragaláctico.

Pero podemos hacer una verificación más fuerte de esta última afirmación. Considerando que el radio de Larmor en kiloparsecs (kpc) para un núcleo de carga $Z e$ con energía en unidades de $10^{18} \mathrm{eV}$, y que se propaga a través de un campo magnético $B_{\mu G}$ medido en $\mu$ Gauss es

$$
R_{k p c} \approx \frac{E}{Z B_{\mu G}}
$$

Teniendo en cuenta que el campo magnético en nuestra galaxia es $\sim 3 \mu_{G}$, para un protón con energía de $10^{18} \mathrm{eV}$ encontramos que tenemos que el valor 
de $R_{k p c}$ es de alrededor de $0.3 \mathrm{kpc}$, lo que es aproximadamente igual al espesor del disco galáctico. Esto implica que las partículas ultra energéticas no podrían estar confinadas en la galaxia y deberían tener origen extragaláctico.

Del análisis de las deflexiones se observa que el ángulo $\theta$ para pequeñas deflexiones debidas a un campo $B_{\mu G}$ para una partícula de carga $Z$ e y energía $E$ recorriendo una distancia $L_{k p c}\left(L_{k p c}\right.$ es la distancia medida en kpc) es

$$
\theta \approx 0.3^{\circ} \frac{L_{k p c} Z B_{\mu G}}{E}
$$

donde hemos usado la aproximación $\operatorname{sen} \theta \approx \theta$. Esta expresión es un resultado inmediato de la ecuación (2.3) usando $L=R \theta$. Para distancias galácticas, se estima que un protón sufre una deflexión de $0.3^{\circ}$ si su energía es de $100 \mathrm{EeV}$.

Consideremos el caso de un protón de $10^{20} \mathrm{eV}$ de procediencia extragaláctica, tenemos que desde una distancia de $50 \mathrm{Mpc}$ la deflexión angular promedio puede ser aproximada como el producto entre $\sqrt{L_{M p c}}\left(L_{M p c}\right.$ es la distancia medida en Mpc) y la desviación angular correspondiente a $1 \mathrm{Mpc}$, es decir $0.3^{\circ}$; consecuentemente tenemos una desviación de $2.1^{\circ}$.

Pero en la región de mayor energía no sólo se produce deflexión en los campos magnéticos. Hay muchos procesos que pueden degradar la energía de una partícula en su propagación asociados a la interacción con los campos de radiación como microondas, infrarrojos y ondas de radio. Inmediatamente después del descubrimiento del fondo cósmico de radiación hecho por Penzias y Wilson (1965) [6], Greisen (1966) [23] y Zatsepin y Kuzmin (1966) [24] predijeron la existencia de un corte en el espectro de protones alrededor de los $5 \times 10^{19} \mathrm{eV}$ principalmente debido a la producción de fotopiones en el fondo de microondas. Su investigación plantea que los protones con energía mayor a $6 \times 10^{19} \mathrm{eV}$ tienen una probabilidad importante de interactuar con los fotones del fondo de radiación cósmico denominados $\gamma_{2.7 K}$, que siguen una distribución Planckiana de $2.7 K$. Este límite en el espectro recibió, en honor a sus descubridores, el nombre de corte GZK.

Para evidenciar la importancia del corte GZK, se observa mediante una simulación (Figura 2.5) la degradación de energía de protones en términos de su distancia recorrida. Puede verse que independientemente de la energía inicial, la energía una vez recorridos $\sim 100 \mathrm{Mpc}$ es en todos los casos menor que $100 \mathrm{EeV}$, lo que limita la distancia de las posibles fuentes de rayos cósmicos con energía del orden de $100 \mathrm{EeV}$.

Las interacciones que dan lugar al corte GZK y las partículas resultantes dependerán obviamente de la naturaleza de la partícula primaria. En el caso de los protones, las principales reacciones son: 


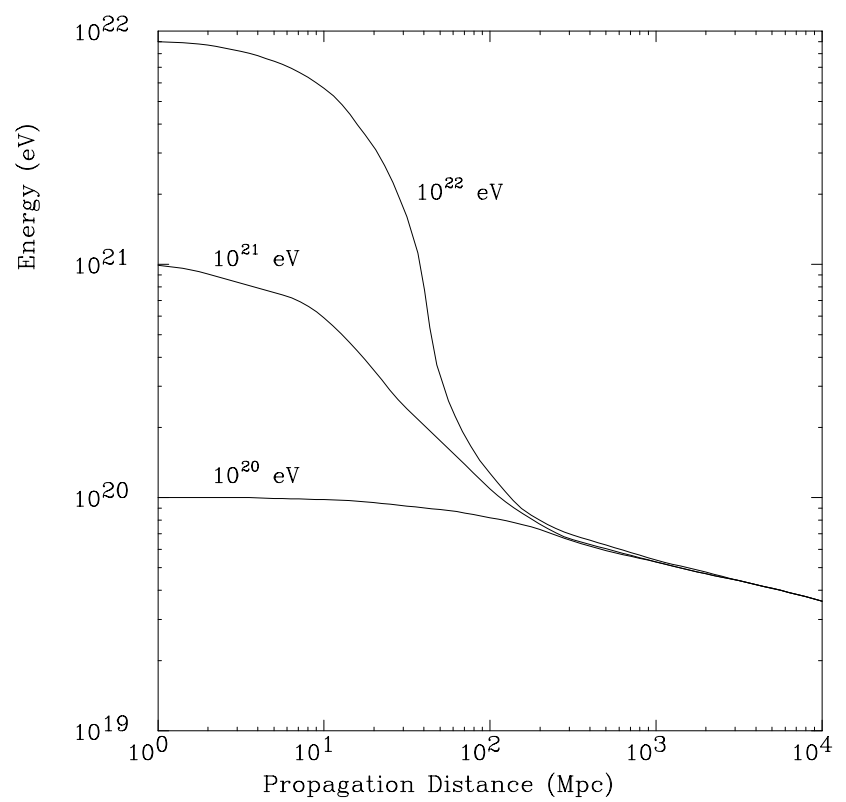

Figura 2.5: Simulación de la pérdida de energía de un protón como función de la distancia de propagación.

$$
\begin{aligned}
p+\gamma_{2.7 K} & \longrightarrow n+\pi^{+} \\
& \longrightarrow p+\pi^{0} \\
& \longrightarrow p+e^{+}+e^{-}
\end{aligned}
$$

Los fotones dominantes del fondo cósmico pertenecen al rango de las microondas con un pico de energía en $6 \times 10^{-4} \mathrm{eV}$ y una densidad de 400 fotones por $\mathrm{cm}^{3}$. Aunque el umbral de energía para la producción de pares es del orden de $10^{18} \mathrm{eV}$ y el camino libre medio es aproximadamente de $1 \mathrm{Mpc}$, comparadas con los $10^{19.6} \mathrm{eV}$ de energía y los aproximadamente $6 \mathrm{Mpc}$ para la producción de piones; la pérdida de energía en la partícula primaria por interacción es del $0.1 \%$ en el caso de una producción de pares y del $20 \%$ en el caso de una producción de piones. Esto significa que si bien es mucho más probable que una partícula con $E>10^{19.6}$ reaccione en una producción de pares, su pérdida de energía no es importante y se verá realmente disminuida cuando la reacción sea una producción de piones.

Los cálculos detallados de la forma del espectro de los rayos cósmicos resultante de considerar la propagación de las partículas a través de ese fondo de radiación, fueron realizados en simulaciones Monte Carlo o métodos analíticos ([25],[26],[27],[28],[29],[30],[31],[32]). 
Si en lugar de protones pensamos en núcleos pesados de masa A, también existen procesos que producen pérdida de energía de ese núcleo. Varios autores analizaron la importancia de la fotodesintegración ([33],[34]) , y la producción de pares [35]. Las principales reacciones que se producen con un núcleo de masa $\mathrm{A}$ y que da como resultados a nucleones $\mathrm{N}$ son:

$$
\begin{aligned}
A+\gamma_{2,7 K} & \longrightarrow(A-1)+N \\
& \longrightarrow(A-2)+2 N \\
& \longrightarrow A+e^{+}+e^{-}
\end{aligned}
$$

Los principales canales son $(\gamma, n)$ y $(\gamma, p)$. La principal pérdida de energía ocurre con la producción de un solo nucleón, que arroja un resultado de un orden de magnitud mayor de energía perdida que en el proceso que incluye dos nucleones $(\gamma, 2 n),(\gamma, n p)$ y $(\gamma, 2 p)$. Recientemente fue reevaluada la magnitud del flujo de radiación infrarroja de fondo [36] basándose en datos experimentales recogidos de 3000 galaxias por el Satélite Astronómico de Infra-Rojo (IRAS). Sus conclusiones fueron que este flujo es un orden de magnitud menor al que se pensaba [34]. La pérdida de energía debido a interacciones con fotones infrarrojos es sólo efectiva por debajo de los $5 \times 10^{19}$ $\mathrm{eV}$, mientras que la energía perdida en interacciones con fotones del fondo de microondas es más significativa para energías de $2 \times 10^{20} \mathrm{eV}$ ([37],[38]).

Si suponemos como partículas primarias a $\operatorname{los}$ rayos $\gamma$, se demostró [36] que el principal proceso que lleva a la pérdida de energía para fotones $\gamma$ mayores a $10^{14} \mathrm{eV}$, es

$$
\gamma+\gamma_{2.7 K} \longrightarrow e^{+}+e^{-}
$$

o sea la creación de pares a partir de la interacción con el fondo cósmico de radiación.

En la actualidad hay nuevos indicios referidos a la existencia del corte GZK. Del trabajo del Observatorio Pierre Auger anteriormente mencionado [58], particularmente observado en la Figura 2.2, se desprende también una conclusión respecto al GZK. En comparación a la extrapolación de la ley de potencias, el espectro está suprimido por un factor 2 cuando $\log _{10}\left(E_{1 / 2} / \mathrm{eV}\right)=19,61 \pm 0,03$. Esta supresión es similar a la esperada por el efecto GZK para protones o núcleos pesados, pero puede en parte ser relacionado con un cambio de la forma de inyeccin media de las fuentes. 


\subsection{1. ¿Isotropía o Anisotropía?}

La búsqueda de las fuentes de los rayos cósmicos ultraenergéticos tiene muchos antecedentes. Incluso cuando se han identificado muy buenos candidatos para las regiones de acleración, como las radio galaxias cercanas [68] con sus núcleos galácticos activos (AGN) posibles contenedores de agujeros negros super-masivos con masas 6 órdenes de magnitud mayores a la solar. En estos núcleos galácticos se producen fenómenos de acreción con grandes cantidades de materia involucradas y parte de ésta es expulsada en forma de jets.

De acuerdo a las predicciones teóricas, enmarcadas en los modelos bottomup, se considera que estos objetos pueden acelerar las partículas hasta alcanzar energías del orden de $10^{21} \mathrm{eV}$ [69]. Se estima que los sitios de aceleracin se encuentran en los finales de los jets de AGN, donde el jet golpea una nube intergaláctica de la materia [34], o en la región muy cercana al agujero negro [70] e incluso en los restos de otros jets de AGN [71].

Sin embargo las mediciones no permiten una evidencia tan determinante. Los eventos detectados con energías mayores a $10^{19} \mathrm{eV}$ muestran una distribución de direcciones de arribo isotrópicas. A pesar de ello AGASA reportó además la existencia de "clusters", es decir una serie de eventos correlacionados a un origen común, en los eventos con $E>4 \times 10^{19} \mathrm{eV}$ [21]. La confirmación de estos multipletes daría información sobre posibles fuentes y acerca de los campos magnéticos en la región de propagación. HiRes ha intentado confirmar este hallazgo sin resultados positivos, concluyendo que hasta el presente no hay evidencia suficiente para determinar anisotropía en las direcciones de arribo [22].

Los últimos resultados del Observatorio Pierre Auger sugieren una posible correlación entre las direcciones de arribo de rayos cósmicos de energía superior a 57 EeV y la ubicación de entornos de AGNs [67]. El trabajo plantea que 22 eventos de 27 , se encuentran dentro de un ángulo de separación de $3.1^{\circ}$ de las AGN, y la mayoría de las no correlaciones se encuentran sobre el plano galáctico, donde se esperan deflexiones muy grandes debido al campo magnético galáctico. Es importante aclarar que el trabajo no concluye que las AGN son candidatos de fuentes de rayos cósmicos ultraenergéticos, sino que establece que es el entorno de las AGN (donde se conoce que hay múltiples objetos celestes distribuidos) quien es un posible sitio de generación de los mismos. 


\section{Capítulo 3}

\section{Los Rayos Cósmicos en la Atmósfera}

Si nos detenemos a pensar en el volumen del planeta Tierra, comparativamente al sistema solar o la galaxia misma; a primera vista tal vez concluyamos que hay una baja posibilidad de llegada de los rayos cósmicos a nuestro planeta. Sin embargo la producción de estas partículas es tan numerosa que luego de haberse propagado a través del espacio, un número no despreciable de ellas arriba a la Tierra. Esto sugiere que un posible estudio de los rayos cósmicos estaría ligado a una medida directa obtenida a partir de detectores situados en la estratósfera, por ejemplo en satélites o la estación internacional espacial. Pero como el flujo de las partículas ultraenergéticas resulta tan bajo, el arreglo de detectores debería tener entonces un área significativa; y si consideramos además el costo económico asociado a la puesta en funcionamiento y mantenimiento de una estructura en el espacio, este tipo de estudios no resultan muy accesibles.

Los experimentos de rayos cósmicos suelen aprovechar entonces los efectos de la llegada de estas partículas a la atmósfera terrestre. A partir del ingreso de las partículas primarias en la atmósfera, se produce una serie de interacciones con la materia presente en la atmósfera, fenómeno denominado extensive air shower (EAS), que suele ser traducido como cascada de partículas o lluvia de partículas. Este fenómeno, consiste en que la partícula primaria interacciona con la materia presente en la alta atmósfera, con energía suficiente para crear nuevas partículas, ellas también con energía suficiente para avanzar y crear nuevas partículas. Este proceso se repite en el avance de esas partículas hasta que la energía disponible no permite nuevas creaciones de partículas y se producen fenómenos de absorción y pérdida de energía. La energía de las partículas primarias es de tal magnitud que los procesos implicados en la creación de nuevas partículas generan del orden de $10^{11}$ nuevas 
partículas y una importante porción de ellas puede lograr alcanzar el nivel de la superficie terrestre.

Mediante el análisis de la fenomenología de estas cascadas de partículas secundarias, es entonces que los experimentos de rayos cósmicos estudian las características de las partículas primarias de ultra-alta energía.

Estudiaremos en este capítulo las características de la evolución de las cascadas; los parámetros involucrados y su detección.

\subsection{Evolución de las cascadas}

La atmósfera como gran volumen de materia para interacciones tiene, entre otras, las siguientes características que podemos enumerar:

- Su baja densidad atómica permite una extensa dispersión de los secundarios.

- El espesor vertical es suficiente para que la cascadas alcancen el desarrollo máximo de partículas, permitiendo relacionar proporcionalmente el número total de partículas con la energía total.

- La inhomogeneidad del perfil de densidad permite establecer para su estudio las diferentes zonas de desarrollo de las cascadas donde se producen los procesos de multiplicación y absorción de partículas. La relación que describe la variación de la densidad con la atmósfera se obtiene haciendo medidas a distintas alturas sobre el nivel del mar.

El análisis de la cascada en la superficie terrestre conlleva la desventaja de no tener medidas directas de la partícula primaria ni de las primeras interacciones. Sin embargo a partir del estudio de las partículas secundarias dispersadas en grandes áreas, se puede inferir la naturaleza de la partícula primaria y su comportamiento en las primeras interacciones.

La cascada generada por la interacción entre la partícula primaria y la alta atmósfera tiene la forma aproximada, en cada instante de tiempo, de un disco centrado en el eje de la lluvia que va aumentando su diámetro a medida que avanza. Este disco se mueve prácticamente a la velocidad de la luz, y adquiere su máxima extensión cuando coexisten el mayor número de partículas secundarias, en lo que se conoce como el máximo de la lluvia. La profundidad atmosférica correspondiente a este máximo se denomina $X_{\max }$. Tras alcanzar este desarrollo máximo de partículas, el número de partículas se atenúa a medida que ellas no tienen la suficiente energía como para continuar la producción de nuevas partículas. 
Es importante destacar que en realidad el disco posee un leve espesor y pequeñas curvaturas convexas en sus caras (en la cara frontal la curvatura es menos significativa) debido al tipo de partículas generadas. El espesor del disco medido en tiempo es de unos nanosegundos en el centro y microsegundos en los extremos. Este espesor está medido en tiempos porque representa la diferencia temporal entre las primeras y las últimas partículas en cada instante de la lluvia avanzando.

Si pudiéramos observar el frente de la cascada en un instante observaríamos que ese disco concentra cercano al eje a muones que mayoritariamente avanzarán sin interactuar y a numerosos electrones y rayos $\gamma$ originados por partículas cercanas al eje. A medida que nos alejamos del centro el espesor del disco aumenta producto de partículas ampliamente dispersadas, la parte frontal constituido mayoritariamente por muones, que interactuaron poco; mientras que varios $\mu$ s después se encuentra la última de las partículas electromagnéticas.

Cuando examinamos las partículas involucradas en la lluvia, observamos que en el caso de que la partícula primaria sea un nucleón o un núcleo, la cascada comienza con una interacción hadrónica. A partir de ese punto, debido al tipo de partículas involucradas en la lluvia, podemos identificar dentro de ella a tres componentes de cascada: la componente electromagnética, la componente muónica y la componente nucleónica (Figura 3.1).

Tras la primera interacción, el número de hadrones aumenta en cada generación de interacciones con nuevas partículas; generando la mencionada componente nucleónica. Asimismo en cada creación de hadrones aproximadamente la mitad de la energía continúa en las partículas nucleares, en promedio la mayor parte es llevada por un solo nucleón de alta energía cerca del eje de la cascada. Al nivel del suelo llega entonces un muy pequeño número de nucleones, en proporción al resto de las partículas, pero varios con importante energía.

La componente muónica se origina a partir del decaimiento de los piones cargados producidos a partir de las interacciones hadrónicas en los sucesivas generaciones de partículas Los $\pi^{+}$y $\pi^{-}$de la generación inicial en su mayoría no decaen, sino que realizan nuevas interacciones. Después de algunas generaciones, las energías de los piones cargados individuales ha disminuido lo suficiente, y acorde a su vida media $\left([2.6033 \pm 0.0005] \times 10^{-8} \mathrm{~s}[39]\right)$, es que comienzan a producirse los decaimientos

$$
\pi^{ \pm} \longrightarrow \mu^{ \pm}+\nu_{\mu}
$$

generando la cascada muónica compuesta por muones y sus correspondientes neutrinos. Los muones no se multiplican y se absorben muy lentamente por ionización, con lo cual la mayoría penetra hasta alcanzar el nivel del suelo. 


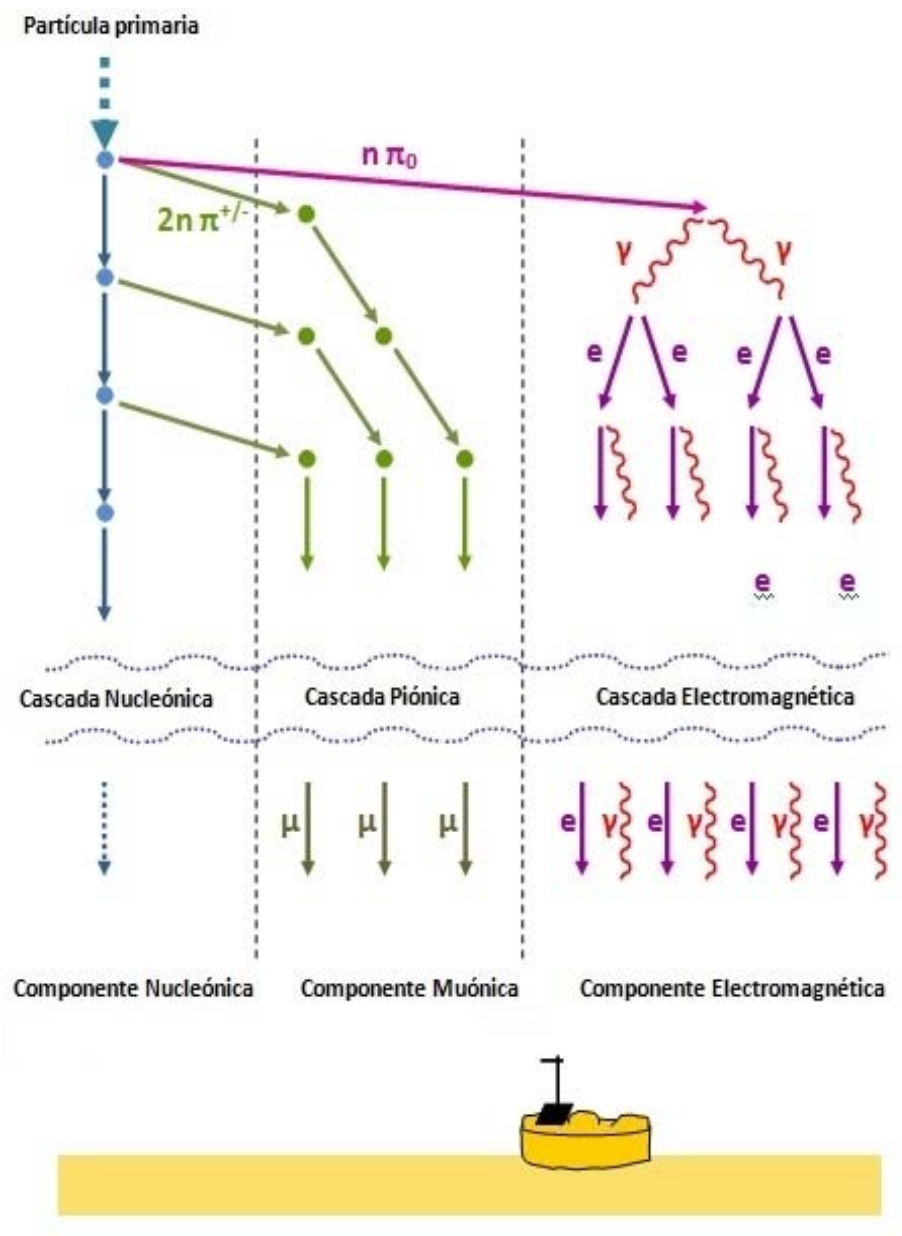

Figura 3.1: Diagrama esquemático mostrando los procesos principales en las cascadas. La componente nucleónica se encuentra concentrada en un área estrecha cercana al core mientras que las componentes muónicas y electromagnéticas cubren un área extensa. Dependiendo del tipo de partícula primaria, la energía y la inclinación del eje la cascada, se observará a nivel del suelo una distinta contribución de cada una de las componentes. 
La cascada electromagnética se produce debido a los numerosos $\pi^{0}$ surgidos también a partir de las interacciones hadrónicas. Estos tienen una breve vida media $\left([8.4 \pm 0.5] \times 10^{-17} \mathrm{~s}[39]\right)$ de manera tal que la gran mayoría, antes de poder interactuar, decae en rayos $\gamma$ de acuerdo a

$$
\pi^{0} \longrightarrow \gamma+\gamma
$$

que a su vez, debido al proceso de creación de pares producto de la interacción con el campo nuclear de los átomos de la atmósfera, generan

$$
\gamma \longrightarrow e^{+}+e^{-}
$$

Éstos leptones $e^{ \pm}$a su vez generan fotones por radiación de bremsstrahlung, nuevamente por la interacción con núcleos de átomos (de número másico A) de la atmósfera

$$
e^{-}+A \longrightarrow e^{-}+A+\gamma
$$

y más fotones por aniquilación

$$
e^{+}+e^{-} \longrightarrow \gamma+\gamma
$$

produciendo una numerosa cantidad de partículas. Estos procesos pueden verse claramente en el diagrama de la Figura ( 3.2)

Pese a que la generación de la componente electromagnética está dominada por creación de pares y bremsstrahlung, eventualmente la energía promedio cae por debajo de un valor crítico $\epsilon_{0}$, en el cual los efectos de ionización son los relevantes en los mecanismos de pérdida de energía. Existen diversas definiciones del valor de $\epsilon_{0}$ [40] de los cuales uno de los más aceptados toma la energía crítica como aquella a la que las pérdidas por longitud de radiación son iguales a la energía del electrón. El valor planteado es

$$
\epsilon_{0}=\frac{710 \mathrm{MeV}}{Z_{e f f}+0.92} \sim 86 \mathrm{MeV}
$$

Se puede entonces categorizar el desarrollo de la lluvia en tres fases, la fase de crecimiento en la cual todas las partículas tienen energía mayor que $\epsilon_{0}$, la fase del máximo desarrollo $X_{\max }$, y la fase correspondiente a la cola de la lluvia dónde las partículas sólo pierden energía.

El número de partículas de la cascada electromagnética que llegan a la superficie es de varios órdenes de magnitud. Por ejemplo, para una lluvia vertical originada por un protón de $10^{11} \mathrm{GeV}$, hay alrededor de $10^{11}$ partículas secundarias con energía por encima de $90 \mathrm{keV}$ que alcanzan la superficie 


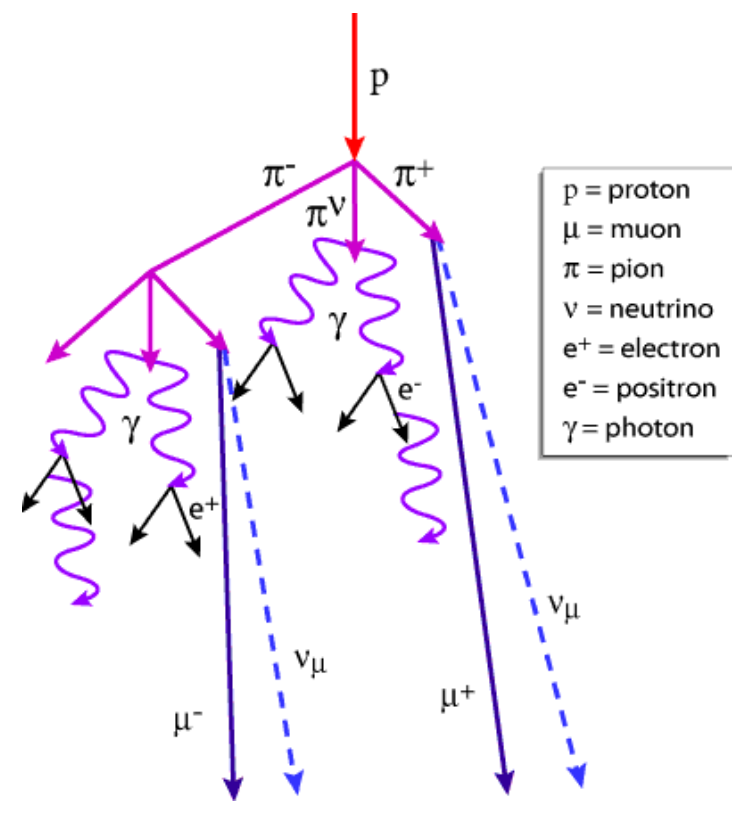

Figura 3.2: Diagrama representando la generación de las nuevas partículas secundarias.

terrestre. De esas partículas el $99 \%$ son fotones, electrones y positrones. $\mathrm{Su}$ energía media es de alrededor de $10 \mathrm{MeV}$ y transportan el $85 \%$ de la energía total que llega al nivel del suelo [41].

Es importante mencionar también que la cascada electromagnética se ve fuertemente influenciada por el ángulo de inclinación de la lluvia. Para un ángulo de $\theta>70^{\circ}$, la componente electromagnética se atenúa exponencialmente siendo completamente absorbida al nivel de la superficie. Para remarcar esto veamos que para una lluvia vertical al nivel del mar la profundidad es de $1030 \mathrm{~g} / \mathrm{cm}^{2}$ y una lluvia horizontal atraviesa 36 veces esa profundidad [42]. Consecuentemente para una lluvia de gran inclinación, la mayoría de la energía que llega a la superficie es aportada por muones.

En cuanto al ya mencionado $X_{\max }$, que representa la profundidad del máximo desarrollo de la lluvia, debemos aclarar que depende de la energía total y de la masa del primario. Esto implica que el valor de $X_{\max }$ en lluvias originadas por un mismo tipo de primario crece coincidentemente aumenta la energía. Para un dado valor de energía se espera que una cascada producida por un núcleo pesado tenga un desarrollo más rápido que el producido por un protón primario. Esto es en parte debido a que los núcleos pesados tienen una sección eficaz mayor y consecuentemente su probabilidad de interacción es mayor, lo cual se traduce en una mayor posibilidad de interactuar antes. 
Incluso puede estudiarse el comportamiento de las cascadas generadas por núcleos pesados como una superposición de las generadas por los nucleones constituyentes. Para eso podemos usar el modelo de superposición como primer estimador del comportamiento medio de algunas propiedades de las lluvias. Podemos entonces por ejemplo aproximar la cascada generada por un núcleo de hierro de 56 nucleones, como una superposición de 56 lluvias originadas por nucleones primarios, donde cada uno de ellos tendría una energía equivalente a la 56-ava parte de la del núcleo de hierro primario. El principio de superposición será comprendido mejor a partir del Modelo de Heitler para cascadas que trataremos seguidamente.

Anticipemos, sin embargo, la cuantificación obtenida por el modelo de superoposición de la afirmación del párrafo anterior que relaciona la composición con el valor de $X_{\max }$. La posición del $X_{\max }$ para lluvias inducidas por protones aumenta en $55 \mathrm{~g} / \mathrm{cm}^{2}$ por cada década de energía, con lo cual al reducir la energía a la 56-ava parte el valor esperado de $X_{\max }$ decrece en aproximadamente $100 \mathrm{~g} / \mathrm{cm}^{2}$. Se espera entonces que un núcleo de hiero con una energía $E$ tenga un $X_{\max }$ de $100 \mathrm{~g} / \mathrm{cm}^{2}$ menor que el $X_{\max }$ de un protón con la misma energía.

\subsection{Modelización de la cascada}

Comentaremos en esta sección el anteriormente mencionado Modelo de Heitler, un simple modelo para la modelización de cascadas. Continuaremos con una de sus exitosas aplicaciones: la determinación teórica de la tasa de elongación (en inglés elongation rate), que marca el cambio de $X_{\max }$ con la energía, y su vinculación en la identificación de la composición de los primarios.

\subsubsection{El Modelo de Heitler}

Para poder deducir relaciones entre los parámetros de la lluvia, Heitler desarrolló un modelo muy simple que sirve como base para inferir conclusiones en el comportamiento de las cascadas electromagnéticas [51]. La idea consiste en modelizar la parte electromagnética de la cascada teniendo en cuenta que de esta manera se describirá a la mayoría de las partículas.

Los principales mecanismos de pérdida de energía en la cascada electromagnética son radiación de bremmstrahlung y producción de pares, por lo que el modelo supone que la partícula primaria atraviesa una cantidad de materia dada por la longitud de radiación $X_{0}$ característica del medio. El valor de $X_{0}$ está dado en buena aproximación por: 


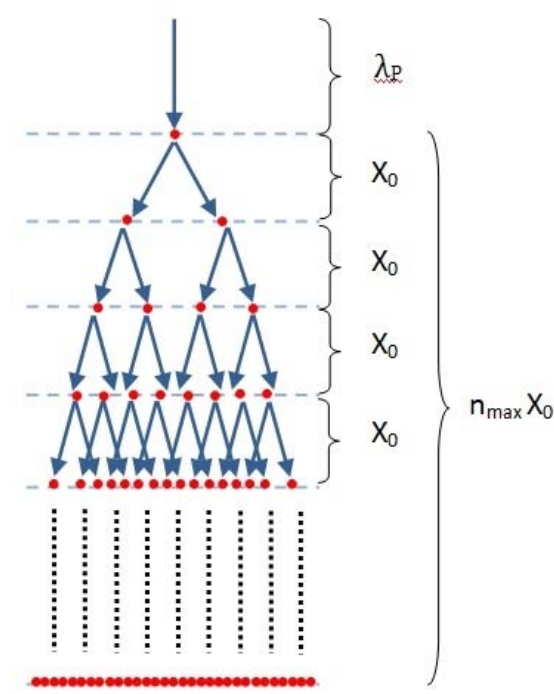

Figura 3.3: Esquema de la generación de partículas secundarias de la cascada electromagnética de acuerdo al Modelo de Heitler.

$$
X_{0}=\frac{716,4 A}{Z(Z+1) \ln \frac{287}{Z}},
$$

donde $Z$ es el número atómico y $A$ es el número másico. Teniendo en cuenta los valores promedio de sus constituyentes tenemos que el valor de $X_{0}$ en el aire es de $36.7 \mathrm{~g} / \mathrm{cm}^{2}$. Ambos procesos producidos en la cascada generan un par de nuevas partículas. A su vez, estas partículas atraviesan una nueva $X_{0}$ generando un nuevo par cada una de ellas (hacer diagrama). Esto implica que de acuerdo a este modelo, transcurridos $n_{\max }$ pasos, la lluvia alcanza su máximo desarrollo produciéndose $N_{\max }=2^{n_{\max }}$ partículas, a una profundidad atmosférica dada por $X_{\max }=X_{0} \cdot n_{\max }$. A su vez, en ese instante cada una de las partículas posee una energía $E_{p} / \epsilon_{0}$, donde $E_{p}$ es la energía del primario y $\epsilon_{0}$ es la mínima energía necesaria para producir un nuevo par de partículas. Teniendo en cuenta estas últimas caracterizaciones podemos expresar a la profundidad a la cual la lluvia alcanza su desarrollo máximo por

$$
X_{\max }=X_{0} \frac{\ln \left(E_{p} / \epsilon_{0}\right)}{\ln 2}
$$

de donde se desprende claramente una dependencia logarítmica con la energía del primario. 
La particularidad de este modelo en relación al mecanismo de superposición es que nos permite inferir una expresión acorde también para núcleos más pesados. Recordando que lo anterior fue obtenido para una lluvia inducida por protones, en el caso de un primario de masa $A$, con energía inicial $E_{p}$ idéntica al caso anterior, podemos pensar que esa energía se encuentra ahora distribuida en $A$ nucleones.

En definitiva esto significa que una lluvia de Núcleo de masa $A$ y energía $E_{p}$ equivale aproximadamente a $A$ lluvias de protones con energía $E=E_{p} / A$.

Aplicando esta idea sobre la expresión 3.8 obtenemos

$$
X_{\max }=X_{0} \frac{\ln \left(E_{p} / A \epsilon_{0}\right)}{\ln 2}=X_{0} \frac{\ln \left(E_{p} / \epsilon_{0}\right)}{\ln 2}-X_{0} \frac{\ln \left(A \epsilon_{0}\right)}{\ln 2}
$$

que representa la estimación del modelo para la profundidad de máximo desarrollo de una lluvia originada por núcleo de masa $A$. Es evidente de esta última expresión, que el modelo reproduce la disminución en el valor de $X_{\max }$ acorde aumenta la masa del primario.

\subsection{2. $\quad$ El Teorema del Elongation Rate}

Anteriormente hemos planteado el tema de la búsqueda en la determinación de la composición de los rayos cósmicos ultraenergéticos como uno de los intereses primordiales de los experimentos de rayos cósmicos. En los párrafos previos comentamos también que las lluvias originadas por primarios de diferente peso atómico, pero de igual energía, producen diferentes desarrollos longitudinales. Particularmente considerando el instante de máximo desarrollo de la cascada, esto nos conduce a pensar a $X_{\max }$ como un buen candidato para identificar composición de la partícula primaria. Con esta idea como base es que surge uno de los más aceptados conceptos para determinar composición derivados del observable $X_{\max }$ : la elongation rate.

Se define la elongation rate como

$$
D_{e}=\frac{\delta\left\langle X_{\max }\right\rangle}{\delta \ln E}
$$

que representa el cambio $\delta\left\langle X_{\max }\right\rangle$ respecto a la energía $\delta \ln E$. Para observar su influencia en la determinación de composición, calculemos los valores correspondiente a lluvias originadas por primarios de diferente masa.

Primero, ampliando la idea sugerida por el Modelo de Heitler, se propone para una lluvia inducida por protones una dependencia de $X_{\max }$ con la energía del tipo 


$$
X_{\max }=\lambda_{p}+X_{0} \ln (E /\langle n(E)\rangle),
$$

donde se ha tenido en cuenta que la lluvia no es puramente electromagnética, por lo que debemos tener en cuenta el punto de primera interacción $\lambda_{p} \mathrm{y}$ la multiplicidad $\langle n(E)\rangle$.

En base a la definición de la elongation rate, obtenemos para esta modelización de la lluvia,

$$
\begin{aligned}
D_{e}= & \frac{\delta\left\langle X_{\max }\right\rangle}{\delta \ln E}=\frac{\delta\left(\lambda_{p}+X_{0} \ln (E /\langle n(E)\rangle)\right.}{\delta \ln E}= \\
& =X_{0}\left(1-\frac{\delta \ln (\langle n(E)\rangle)}{\delta \ln E}+\frac{\lambda_{p}}{X_{0}} \frac{\delta \ln \lambda_{p}}{\delta \ln E}\right)
\end{aligned}
$$

que es la ecuación definida por Linsley y Watson [52]. Parte de la modelización, coherente con la interpretación de los modelos hadrónicos, consiste en asumir como constante el término

$$
B \equiv \frac{\delta \ln (\langle n(E)\rangle)}{\delta \ln E}-\frac{\lambda_{p}}{X_{0}} \frac{\delta \ln \lambda_{p}}{\delta \ln E}
$$

En el caso en que el modelo de las interacciones hadrónicas representa la multiplicidad de la forma $\langle n(E)\rangle=n_{0} E^{\Delta}$, donde $\Delta$ es el factor de multiplicidad, tendremos este último parámetro definido como

$$
B \equiv \Delta-\frac{\lambda_{p}}{X_{0}} \frac{\delta \ln \lambda_{p}}{\delta \ln E},
$$

por lo que arribamos a la expresión para la elongation rate de una lluvia de protones

$$
D_{e}=X_{0}(1-B)
$$

donde es evidente que será una constante cuando $B$ sea modelizada como constante.

En el caso en que la cascada sea producida por un núcleo de peso atómico $A$, apelamos nuevamente al modelo de superposición descrito anteriormente. Una lluvia originada por un núcleo primario de masa $A$ y energía $E=E_{A}$ se representa como $A$ lluvias de protones con energía $E=E_{A} / A$ y longitud de primera interacción $\lambda_{p}\left(E_{A} / A\right)$. Por lo anterior, la multiplicidad correspondiente a la lluvia originada por el núcleo $A$ estará dada por $\langle n(E)\rangle=A n_{0}\left(E_{A} / A\right)^{\Delta}$. Retomando entonces la expresión para la elengation rate, tendremos para una lluvia producida por un primario de masa atómica $A$

$$
D_{e}=X_{0}\left(1-\frac{\delta \ln (\langle n(E)\rangle)}{\delta \ln E}+\frac{\lambda_{p}\left(E_{A} / A\right)}{X_{0}} \frac{\delta \ln \lambda_{p}\left(E_{A} / A\right)}{\delta \ln E}\right),
$$


y operando utilizando las propiedades de la derivación y del logaritmo, y utilizando la expresión (3.13) se llega a

$$
D_{e}=X_{0}\left(1-B-\frac{\delta \ln A}{\delta \ln E}(1+B)\right)=X_{0}(1-B)\left(1-\frac{\delta \ln A}{\delta \ln E}\right)
$$

Es evidente la interpretación de este último resultado en relación a la composición del primario: la elongation rate provee una medida del cambio del logaritmo de la masa promedio con la energía. También se deduce que si el flujo de rayos cósmicos estuviese dominado por una única especie de núcleos, el valor de $D_{e}$ sería constante.

En la práctica se utiliza $D_{10}=2,37 D_{e}$ para expresar en términos de logaritmo base 10 de energía. Los últimos resultados experimentales de $X_{\max }$ [72], presentados como función de la energía de manera tal de apreciar la elongation rate, no evidencian una clara definición respecto a la composición de los primarios. El ajuste sobre las mediciones dieron como resultado $D_{10}=$ $106_{-21}^{+35} \mathrm{~g} \mathrm{~cm}^{-2}$ para energías menores a $10^{18,24 \pm 0,05} \mathrm{eV}$, mientras que para energías mayores a este punto el resultado es $D_{10}=24 \pm 3 \mathrm{~g} \mathrm{~cm}^{-2}$.

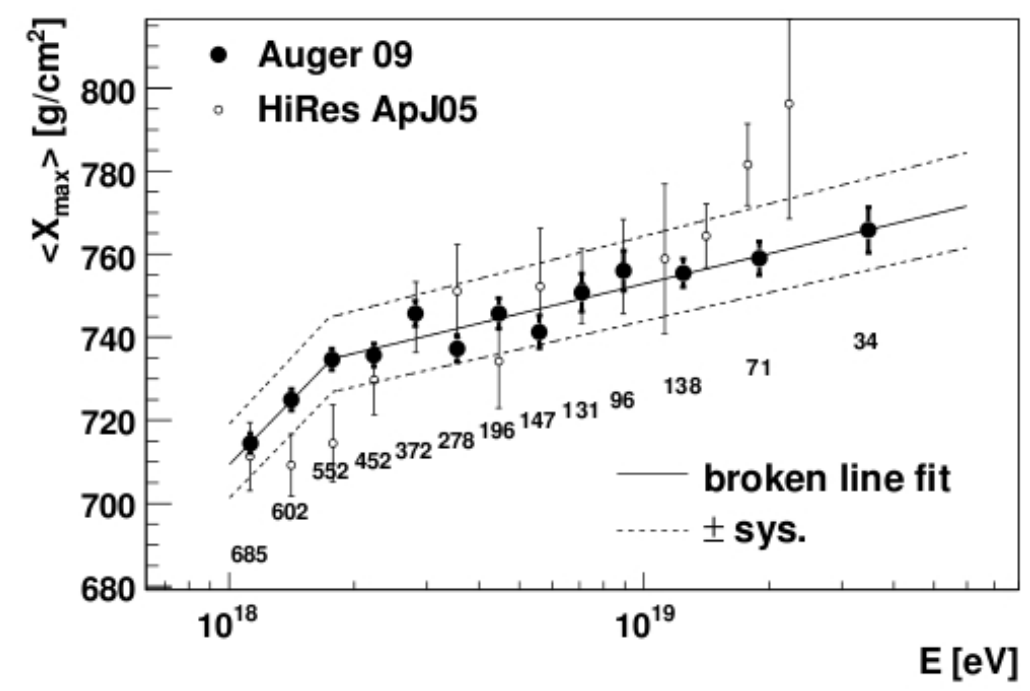

Figura 3.4: Variación de $X_{\max }$ con la energía y visualización de la elongation rate. Últimos resultados experimentales del Observatorio Pierre Auger [72] y el ajuste sobre los datos. El número de eventos asociado a cada medida se muestra debajo de cada una de ellas. Se observa también, a modo de comparación, los valores reportados por el experimento HiRes. 


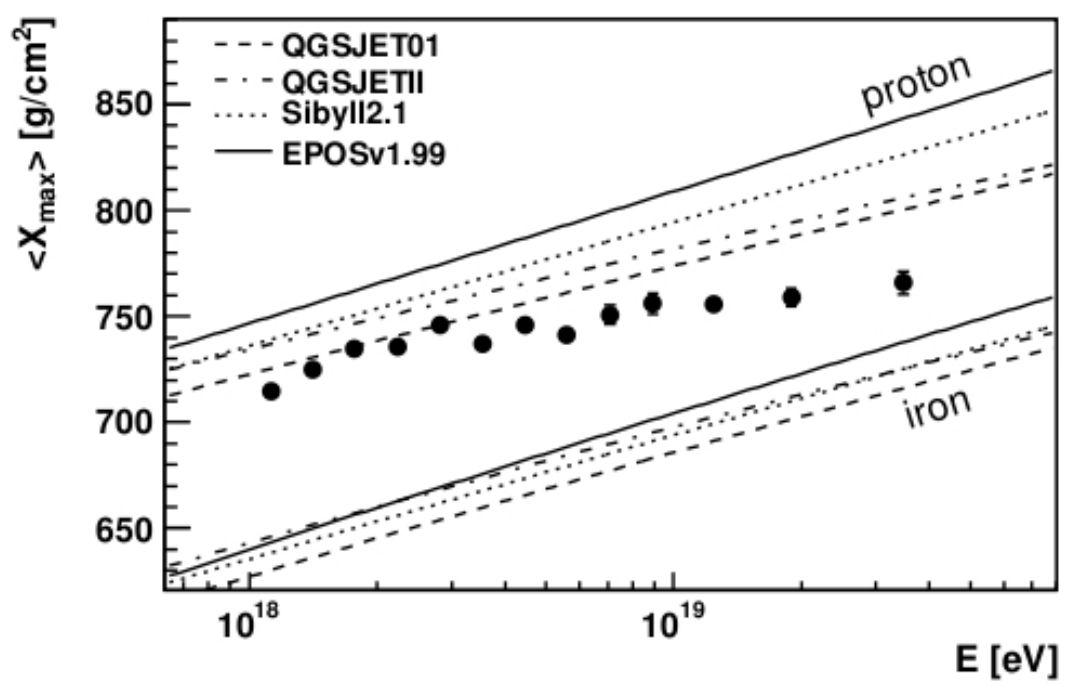

Figura 3.5: Variación de $X_{\max }$ con la energía y visualización de la elongation rate. Últimos resultados experimentales Observatorio Pierre Auger [72] y comparación con las predicciones de las simulaciones para protón y hierro (iron) de acuerdo a varios modelos hadrónicos.

\subsection{Detectores de Superficie: Determinación de los parámetros de la lluvia}

En esta sección explicaremos cuales son los parámetros principales a determinar para las lluvias de ultra-alta energía inducidas en la atmósfera. Haremos un primer hincapié en aquellos parámetros obtenidos mediante detectores de superficie (SD) para luego referirnos en la sección siguiente al caso de los detectores de fluorescencia. No ahondaremos aquí en las características particulares de los detectores de superficie, pero recordemos que esta tesis de investigación se inscribe en el Observatorio Pierre Auger, donde los detectores de superficie son del tipo Cherenkov con agua superpura y tres fotomultiplicadores por tanque. Un desarrollo más exhaustivo de estos detectores se encuentra en el siguiente capítulo.

\subsection{1. $\quad$ El eje y la dirección de la lluvia}

La dirección del eje de la cascada es la misma que la del rayo cósmico primario. En el caso de arreglos de detectores de superficie, esta dirección es deducida de los tiempos de arribos relativos de las señales de al menos tres 
detectores no alineados. Con la señal dejada en estos tres detectores, por una sencilla triangulación, se consigue una primera aproximación del centro de la única lluvia cuyo frente viajando a la velocidad de la luz tiene esas tres posiciones y tiempos de llegada.

Como ya fue planteado, las partículas se dispersan en un disco que aumenta en su radio hasta el momento en que se llega a un máximo desarrollo. Si el arreglo de detectores se encuentra a una profundidad adecuada, podrá detectar distintas señales en una extendida zona. Es por esta razón que los arreglos de superficie diseñados para detectar rayos cósmicos de energía mayor a $10^{19}$ $\mathrm{eV}$ deben construirse en regiones de altura comprendida en los equivalentes $750 \mathrm{~g} / \mathrm{cm}^{2}$ y los $1020 \mathrm{~g} / \mathrm{cm}^{2}$. Esto es adecuado ya que la profundidad máxima promedio de lluvias provocadas por primarios con esas energías ocurre a alrededor de $750 \mathrm{~g} / \mathrm{cm}^{3}$ y para obtener valores precisos en la determinación de los parámetros es conveniente estudiar la lluvia desarrollada después del valor de $X_{\max }$.

La mayor densidad de partículas se genera en la zona cercana al eje, y a medida que uno se aleja de él el número de partículas decrece sustancialmente. Los estudios de simulación de lluvias verticales predicen que la distribución es axialmente simétrica en el plano perpendicular al eje. Este punto ha tenido numerosas comprobaciones experimentales para un amplio rango de energía [43]. A modo de ejemplo se muestra la representación de un evento con estimaciones en energía de $21.7 \pm 1.4 \mathrm{EeV}$ e inclinación 5.2 $\pm 0.4^{\circ}$, registrado con el arreglo de superficie del Observatorio Auger [44] (Figura 3.6).

La posición del eje de la cascada se determina aprovechando esta simetría circular en la densidad de las partículas cuando la lluvia es vertical. En el caso en que la lluvia no sea vertical, la posición del eje, el ángulo zenital $\theta$ y el azimutal $\phi$, se determinan a partir de la ubicación de los detectores y la señal en cada uno de ellos. Utilizando la posición del centro y las densidades de señales, la distancia lateral al eje de la lluvia puede ser estimada para cada punto del arreglo. La reconstrucción se logra ajustando mediante métodos probabilísticos las densidades registradas en los detectores a los valores de una función de distribución lateral empírica - sobre la cual luego nos explayaremos- que permita reajustar la posición más probable del eje y el tamaño de la lluvia.

Una vez determinada la posición del eje es posible reajustar los tiempos de llegada al detector permitiendo la curvatura del frente de la lluvia, y que para la primera aproximación fue considerado plano. Esto provee una dirección más precisa y puede repetirse el proceso con estos nuevos datos.

Veamos más detalladamente cómo es el proceso de la reconstrucción angular [45]. El objetivo es determinar con precisión la dirección $(\theta, \phi)$. Como 


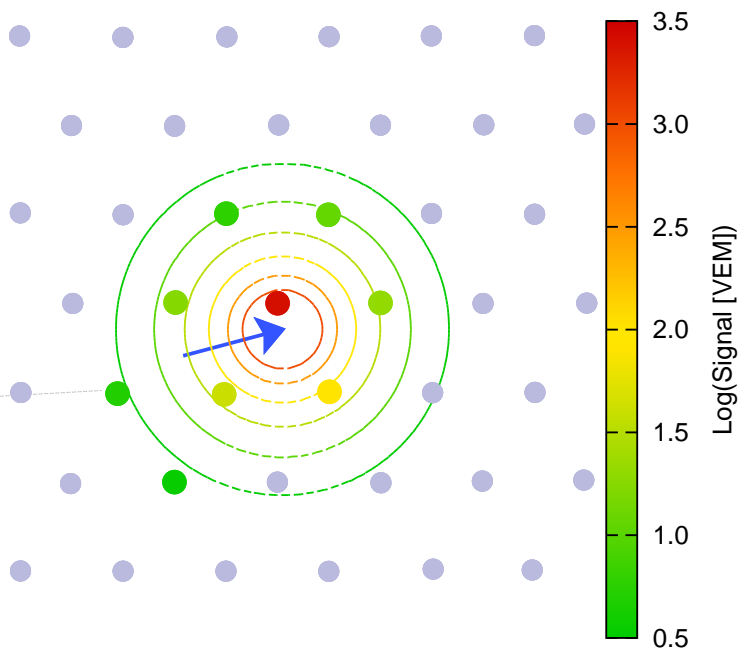

Figura 3.6: Evento 10998100 detectado el 26 de enero de 2011 con el arreglo de superficie del Observatorio Pierre Auger. La flecha señala la posición estimada del core, los puntos la posición de los detectores Cherenkov y la escala cromática representa la intensidad de la señal en los detectores. Notar la distribución simétrica con respecto al eje. 


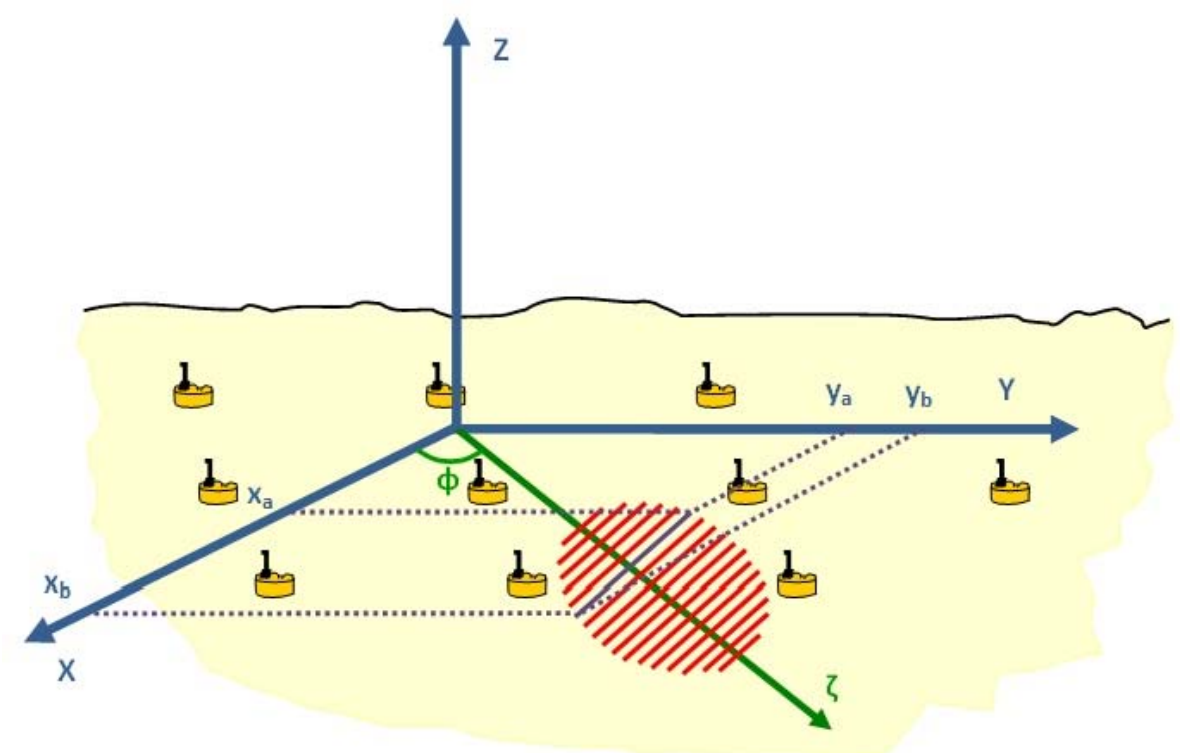

Figura 3.7: Diagrama representativo de la llegada del disco de lluvia y esquema del arreglo que permite la reconstrucción angular.

punto de partida se supone en la reconstrucción que el disco de la lluvia es plano, y conociendo las coordenadas de cada detector en el plano del arreglo $\left(x_{i}, y_{i}\right)$ que pueden en la realidad ser determinadas con precisión de hasta $1 \mathrm{~m}$ en la actualidad gracias al uso de GPS. Entonces al suelo llegan simultáneamente un grupo de partículas formando unas "líneas equitemporales $\tau_{i} "$ (Figura 3.7).

En base a geometría vemos que para los puntos $\left(x_{a}, y_{a}\right)$ y $\left(x_{b}, y_{b}\right)$ ubicados sobre la misma equitemporal se cumple

$$
\tan \phi=\frac{\sin \phi}{\cos \phi}=\frac{x_{a}-x_{b}}{y_{a}-y_{b}}
$$

o sea que

$$
x_{a} \cos \phi-x_{b} \cos \phi=y_{b} \sin \phi-y_{a} \sin \phi
$$

y reagrupando

$$
x_{a} \cos \phi+y_{a} \sin \phi=x_{b} \cos \phi+y_{b} \sin \phi
$$

Pero esto es válido para todos los puntos sobre la misma equitemporal $\tau_{i}$, determinando una constante $\zeta_{i}$ para cada equitemporal, o sea

$$
x_{i} \cos \phi+y_{i} \sin \phi=\zeta_{i}
$$




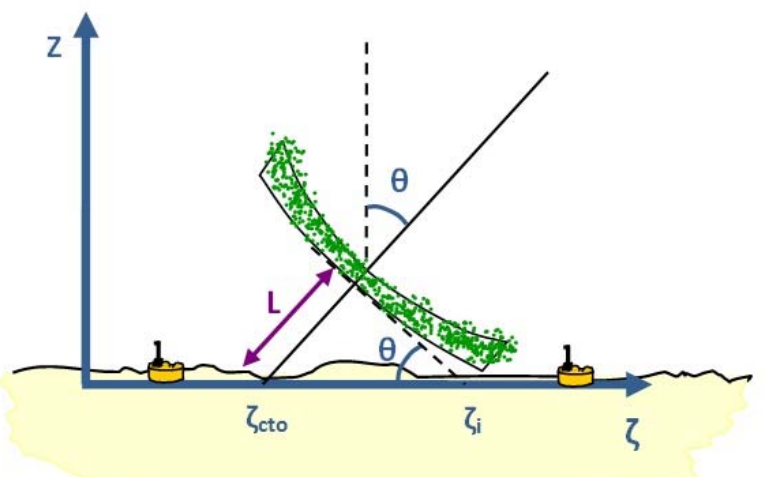

Figura 3.8: Corte transversal del disco de la lluvia observado en el plano $\zeta$ Z

Como podemos relacionar claramente a $\zeta$ con la dirección provista por el ángulo $\phi$, tenemos la posición de la equitemporal $\tau_{i}$ en la dirección $\zeta$, que es la correspondiente al eje trazado en la dirección $\phi$. Llamando $\zeta_{\text {cto }}$ a la equitemporal correspondiente al centro del disco (Figura 3.8), $T_{\text {cto }}$ al tiempo de arribo en el centro de la lluvia de coordenadas $\left(X_{\text {cto }}, Y_{\text {cto }}\right)$, y $t_{i}^{e s p}$ el tiempo esperado de arribo de la partícula a un detector situado en $\left(x_{i}, y_{i}\right)$; tenemos que la diferencia de tiempos entre $T_{c t o} \mathrm{y} t_{i}$ es

$$
t_{i}^{e s p}-T_{c t o}=\frac{L}{c}=\frac{\left(\zeta_{i}-\zeta_{c t o}\right) \sin \theta}{c}
$$

Mediante operaciones algebraicas triviales y el uso de los cosenos directores $u$ y $v$ dados por

$$
u=\sin \theta \cos \phi \quad, \quad v=\sin \theta \sin \phi
$$

se arriba fácilmente a

$$
t_{i}^{e s p}=T_{c t o}+\frac{\left(x_{i}-X_{c t o}\right) u+\left(y_{i}-Y_{c t o}\right) v}{c}
$$

El tiempo real en que se dispara la señal de arribo en cada detector será $t_{i}$. La diferencia de tiempo $\Delta t_{i}$ entre el tiempo de comienzo de la señal, y el esperado por la teoría acorde a los parámetros $u$ y $v$ para una dada geometría de lluvia puede ser escrito como:

$$
\Delta t_{i}=t_{i}-t_{i}^{e s p}=t_{i}-\left(T_{c t o}-\frac{u\left(x_{i}-X_{c t o}\right)+v\left(y_{i}-Y_{c t o}\right)}{c}\right)
$$


La posición en el suelo del centro de la lluvia $\left(X_{\text {cto }}, Y_{\text {cto }}\right)$ se determina por el baricentro de todos los tanques que dispararon señal, pesada por la raíz cuadrada de sus señales, que en el caso de las partículas primarias de muy alta energía posee una precisión típica de $150 \mathrm{~m}$.

Los parámetros que nos interesaba ajustar, $T_{\text {cto }}, \theta$ y $\phi$ se obtienen minimizando la expresión

$$
\chi^{2}=\sum_{i}^{n} \frac{\left(\Delta t_{i}\right)^{2}}{\left(\sigma_{t_{i}}\right)^{2}}
$$

donde $\sigma_{t_{i}}$ representa la incerteza al medir el tiempo de llegada $t_{i}$. Más adelante haremos un análisis más completo acerca de lo que representa $\sigma_{t_{i}}$.

El ajuste angular está fuertemente basado en las medidas del tiempo de arribo $t_{i}$. En las medidas experimentales se busca una gran muestra de datos colectados que permitan estudios detallados de tiempos de medidas hechas por detectores de superficie, y su impacto en la reconstrucción angular.

La sincronización de tiempo entre detectores es de alta importancia, y se realiza analizando los trazos de muones singulares, que son usados como calibración por mostrar una señal limpia con risetime de alrededor de 10 ns.

\subsubsection{La Energía de la partícula primaria}

Los detectores de superficie también permiten estimar la energía del primario a partir de interpolar y/o extrapolar cantidades relacionadas a las señales en los detectores. La unidad de medida elegida para expresar la señal recolectada por una estación de superficie es el muón vertical equivalente (Vertical Equivalent Muon - VEM) [73], que corresponde a la señal promedio que deja un muón atmosférico que atraviesa verticalmente el tanque por su centro.

El arreglo experimental da información de la densidad de partículas en los puntos correspondientes a la localización de los detectores. A estos puntos se le ajusta una función $S(r)$ que representa en el caso de Auger, la señal en los detectores Cherenkov como función de la distancia al eje $r$ y se la conoce como función de distribución lateral. Conocidos los parámetros que regulan $S(r)$, la energía del primario se estima evaluando esa función de densidad a una dada distancia del centro.

Aunque el desarrollo longitudinal fluctúa en cada lluvia, y consecuentemente el número $\mathrm{N}$ de partículas al nivel de la superficie dado por

$$
N=\int 2 \pi \rho(r) d r
$$

fluctúa también considerablemente, se ha planteado [46] que la fluctuación de la densidad de partículas de las lluvia muy lejos del centro es muy 
grande debido a que las medidas de la densidad de partículas son bajas y las fluctuaciones son proporcionalemnte mayores. Muy cerca del centro, las fluctuaciones son también grandes debido a la gran fluctuación en el punto de primera interacción. Pero hay una distancia óptima al centro de la lluvia dónde las fluctuaciones son mínimas. Esta distancia se estima para cada arreglo de acuerdo a su espaciamiento entre detectores, por ejemplo en el caso de Haverah Park y AGASA era $r_{0}=600 \mathrm{~m}$ y en el caso del Observatorio Auger $r_{0}=1000 \mathrm{~m}$.

Diversas simulaciones Monte Carlo de desarrollos de cascadas han confirmado esto y ahora es ampliamente aceptado que la densidad lejos del eje de la lluvia depende levemente del modelo de interacción hadrónica o de la composición del primario, y puede realmente ser usado como estimador de la energía.

Una estimación preliminar de la energía del primario es [47]:

$$
E_{0}[e V]=0.12 \times 10^{18} \times\left(\sqrt{1+11.8(\sec \theta-1)^{2}} S(1000)_{A U G E R}\right)^{1.05}
$$

donde la fución lateral de distribución $S(r)$ está medida en VEM. De acuerdo a esta expresión, una lluvia vertical producida por un primario de energía $10^{19} \mathrm{eV}$ produciría a $1000 \mathrm{~m}$ una señal de 67.5 VEM.

\subsubsection{La función de distribución lateral}

Con el objeto de complementar la señal en los detectores de superficie, se construye la función de distribución lateral (LDF): una función que refleja el desarrollo lateral de la lluvia al nivel del suelo. Esta funcion ajusta con sus parámetros libres a la señal integrada de los detectores SD.

Diversa información puede obtenerse del ajuste resultante. Además de la energía, obtenida mediante la anterior ecuación, la LDF permite conocer la estructura de la lluvia. Ésta información surge a partir de que primarios de diferente composición, desarrollan su máximo de lluvia a diferentes alturas y consecuentemente esto determina la distribución de las partículas que llegan a la superficie. Las funciones de distribución lateral reflejan esta situación ya que las pendientes que marcan el decrecimiento son mucho menos pronunciadas para estos casos. De manera similar se encuentra que para lluvias desarrolladas a mayor profundidad las funciones de distribución lateral poseen un decrecimiento mas marcado.

Por todo lo anterior resulta de gran importancia encontrar la función de distribución lateral que mejor describa los datos. Un grupo de la colaboración del Observatorio Pierre Auger, a partir de simulaciones de lluvias realizadas con AIRES/QGSJETS01 ([100], [101], [102]) en el rango $E=1-100 \mathrm{EeV}$ 
$\left(1 E e V=10^{18} \mathrm{eV}\right)$, y para $\theta=0-60^{\circ} \mathrm{y}$ procesadas con programas de reconstrucción [48]; parametrizaron la función lateral de distribución como $S(r)=E^{0.95} \cdot 10^{A+B x+C x^{2}}$ con $x=\log (r / 1000)$ y determinaron los parámetros A, B y C como función de $\theta$.

Además de las simulaciones, dedujeron la función lateral de distribución a partir de los primeros datos experimentales del Observatorio Auger [49]. Se utilizaron eventos con dirección menor a $60^{\circ}$, y con al menos 6 detectores que detectaran señales mayores a 3 VEM. Se aproximaron como funciones laterales de distribución tres posibles candidatas:

- Una simple Ley de Potencias:

$$
S(r)=S(1000)(r / 1000)^{-\nu}
$$

donde la dependencia con $\theta$ está en el índice $\nu=a+b \sec \theta$

- Una función tipo NKG (Nishimura-Kamata-Greisen):

$$
S(r)=\text { const. }\left(\frac{r}{r_{s}}\right)^{-\beta-0.2} \cdot\left(1+\frac{r}{r_{s}}\right)^{-\beta}
$$

donde $\beta=\mathrm{a}+\mathrm{b}$ sec $\theta$ y $r_{s}=700 \mathrm{~m}$.

- Una función inspirada en las simulaciones

$$
S(r)=E^{0.95} \cdot 10^{A+B x+C x^{2}}
$$

Los resultados demostraron que dentro de los errores no hay una tendencia aparente que permita elegir una de las funciones como la mejor. Lo que significa que todas describen correctamente los datos disponibles. Sin embargo estudios previos [50] demuestran que una ley de potencias pura no describe las señales a grandes distancias del centro de la lluvia.

En el caso del Observatorio Pierre Auger, se ha oficializado el uso de una función del tipo NKG de la forma (3.29) tanto para simulaciones como para análisis de eventos. Sobre este punto volveremos en el capítulo 8.

\subsection{Detector de Fluorescencia: Determina- ción de los parámetros de la lluvia}

Teniendo en cuenta las características híbridas del Observatorio Pierre Auger, es que desarrollaremos en esta sección la manera en que se determinan los parámetros de las lluvias mediante los detectores de fluorescencia 
(FD). Al igual que respecto a los detectores de superficie, una descripción pormenorizada de los detectores de fluorescencia se encuentra en el capítulo siguiente.

Anticipemos sólo lo necesario para comprender esta sección: los detectores FD están compuestos por cámaras de fotomultiplicadores (PMT's) que detectan la luz de fluorescencia emitida por las desexcitaciones de átomos de Nitrógeno del aire producidas tras la interacciones con las partículas secundarias y la luz de efecto de Cherenkov por la propagación de las partículas en la atmósfera.

\subsubsection{Geometría de la lluvia}

La reconstrucción de la lluvia medida con los detectores de fluorescencia comienza con un algoritmo que determina la importancia de señal en los fotomultiplicadores por sobre el ruido.

Habiendo determinado que la señal en los PMT (cada PMT actúa como un píxel de la cámara) corresponde a un evento, se comienza con la reconstrucción de la geometría; utilizándose para ello el pulso medido de tiempo en cada píxel $i\left(t_{i}^{\text {med }}\right)$, definido como el centroide del pulso encontardo; y la señal total integrada de ese píxel $\left(w_{i}\right)$. Un nuevo algoritmo determina cuáles son los píxeles vinculados a la figura del trazo de la lluvia obtenida en la cámara y descarta nuevos posibles píxeles con ruido.

El paso siguiente es determinar el plano detector-lluvia, shower-detector plane (SDP), es decir el plano que contiene a la lluvia y al trazo de señal en la cámara, para lo cual se utiliza el vector normal de cada píxel $\left(\vec{p}_{i}\right)$ con señal (Figura 3.9). La precisa determinación se logra minimizando la expresión

$$
Q^{2}=\sum_{i} w_{i}\left|\vec{p}_{i} \cdot \vec{n}\right|
$$

donde $\vec{n}$ es el versor normal al plano SDP, considerando que $Q^{2}$ es nulo en el caso ideal. 
Detector de Fluorescencia: Determinación de los parámetros de la lluvia 51

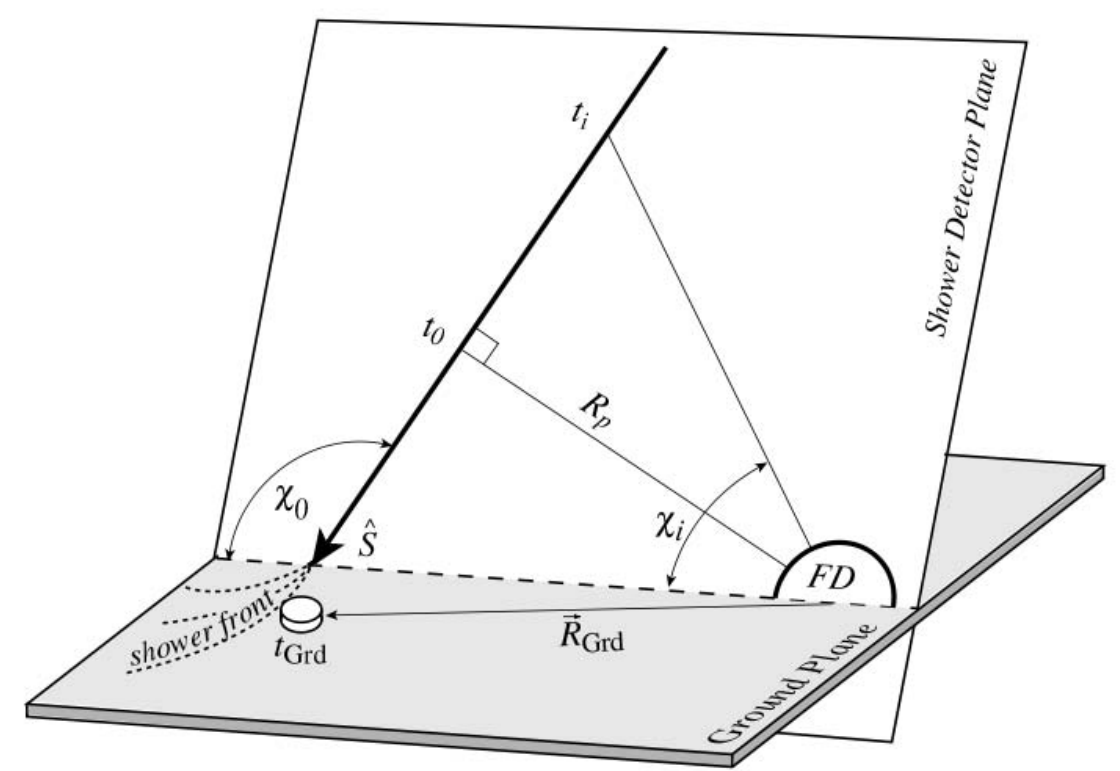

Figura 3.9: Parámetros involucrados en la determinación del Shower Detector Plane

La determinación de la geometría de la lluvia se completa conociendo la posición de la lluvia dentro del SDP, el parámetro de impacto $R_{p}$, el instante de tiempo correspondiente $t_{0}$ y el ángulo entre el eje de la lluvia y la horizontal del suelo $\chi_{0}$. La dirección en que apuntan los píxeles puede ser expresada en términos de cada ángulo de elevación $\chi_{i}$, y se logra minimizando

$$
\chi^{2}=\sum_{i} w_{i}\left(\frac{t_{i}-t_{i}^{\text {med }}}{\sigma_{t_{i}}}\right)
$$

donde $t_{i}$ es la expresión teórica para el pulso de tiempo

$$
t_{i}=t_{0}+\frac{R_{p}}{c} \cdot \tan \left(\frac{1}{2} \cdot\left(\chi_{0}-\chi_{i}\right)\right)
$$

La correlación entre las variables es grande, ya que una pequeña variación en el ángulo de elevación $\chi_{0}$ induce un gran cambio en los otros dos parámetros. Este inconveniente se soluciona incorporando información temporal de un detector de superficie, y es uno de los fuertes de los modo de detección híbridos. Suponiendo que el frente de la lluvia dispara la medida de cada detector de superficie, a un tiempo

$$
t_{G r d}^{m e d}=t_{0}+\frac{1}{c} \vec{R}_{G r d} \cdot \vec{n}
$$


donde $\vec{R}_{G r d}$ es la posición del tanque con respecto al detector de fluorescencia y $\vec{n}$ es la dirección de la lluvia. En las detecciones híbridas, el tanque elegido para esta correlación es el que se encuentra dentro de un círculo de radio de 2 $\mathrm{km}$ centrado en la intersección del eje la lluvia y el suelo. En la mayoría de los casos el tanque con mayor señal es el que suele cumplir estos requerimientos.

\subsubsection{Determinación de la Energía del primario}

Utilizando detectores de fluorescencia, la energía de la partícula primaria se determina aprovechando la fuerte correlación que existe entre ella y la energía depositada en la atmósfera; considerando a su vez que esta última se deduce del número de fotones detectados. Los detectores de fluorescencia, pese a su denominación, perciben además de la luz de fluorescencia de la desexcitación de las moléculas de Nitrógeno, la radiación de fotones Cherenkov, estos últimos producidos porque las partículas se propagan a velocidades mayores que la de la luz en el aire. Estos fotones, de diferentes longitudes de onda, pueden experimentar dispersión Rayleigh y dispersión Mie, y la absorción de la atmósfera. Debido a este punto, resulta de suma importancia el preciso conocimiento de las condiciones locales de la atmósfera en los instantes de medición, y fue esto lo que motivó fuertemente la estructura de este trabajo de investigación.

Ya se comentó en esta subsección que la cantidad de luz de fluorescencia es linealmente proporcional a la energía depositada por las partículas a lo largo de la propagación por la atmósfera, que a su vez es proporcional a la energía del primario. La clave está entonces en establecer el número de fotones que alcanzan los píxeles de la cámara..

El número de fotones de fluorescencia producido por la lluvia está dada por

$$
N_{\gamma}^{f}\left(X_{i}\right)=Y_{i}^{f} \frac{d E}{d X_{i}}
$$

donde $Y_{i}^{f}$ es el fluorescence yield, el factor que establece la producción de fotones de fluorescencia, y $\frac{d E}{d X_{i}}$ refiere a la energía depositada a la profundidad atravesada $X_{i}$.

Cuando los fotones sufren dispersión del tipo Rayleigh o Mie, sólo una fracción $T_{i}$ del total de fotones producidos alcanzan el detector. La luz de fluorescencia emitida alcanza el detector en el tiempo $t_{i}$ (Figura 3.10). Dada la eficiencia $\epsilon$ del detector, y la apertura $A$; el flujo de luz de fluorescencia $y_{i}^{f}$ medido es

$$
y_{i}^{f}=\frac{A \epsilon T^{i}}{4 \pi r_{i}^{2}} Y_{i}^{f} \frac{d E}{d X_{i}}
$$



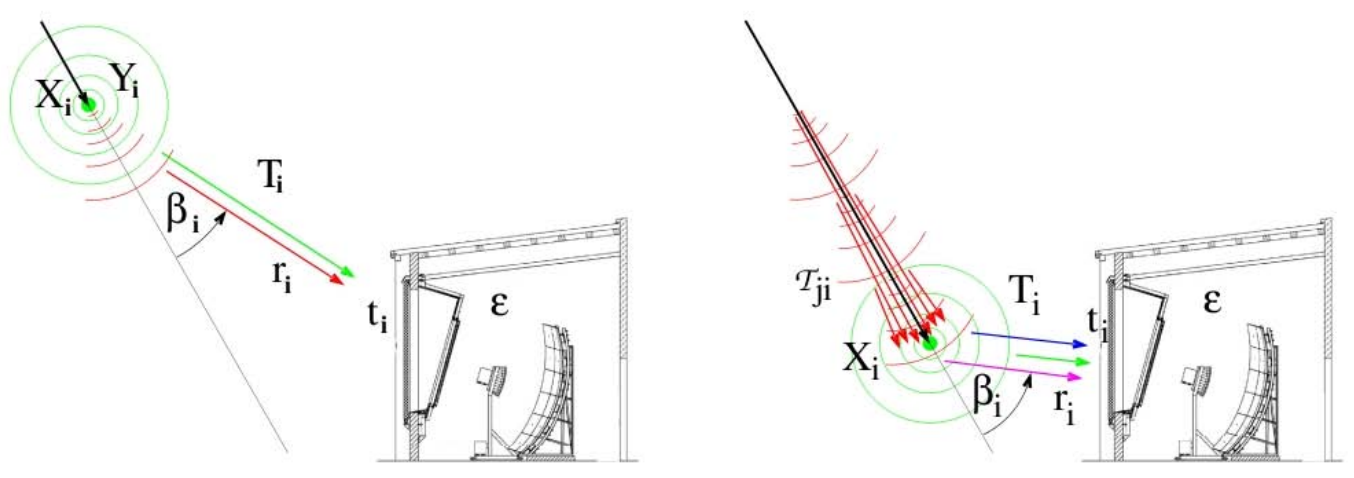

Figura 3.10: Esquema del flujo de luz percibido por los detectores FD, cuando la llegada es directa (izq) y cuando hay dispersión Rayleigh o Mie (der). Se observan las contribuciones al flujo por luz de fluorescencia isotrópica (verde), luz de Cherenkov directa (rojo), luz Cherenkov dispersada por efecto Rayleigh (azul) o Mie (fucsia).

Por otra parte el número de fotones Cherenkov que alcanzan el detector está ligado al factor de producción de los mismos, denominado similarmente Cherenkov yield y representado como $Y_{i}^{C}$. El valor de esta cantidad es también proporcional a $N_{e}\left(X_{i}\right)$ que representa el número de partículas cargadas por encima de una cierta energía, y que es en buena aproximación el número de electrones y positrones. La expresión entonces que define al número de fotones Cherenkov está dada por

$$
N_{\gamma}^{C}\left(X_{i}\right)=Y_{i}^{C} N_{e}\left(X_{i}\right)
$$

Si denominamos $f_{C}\left(\beta_{i}\right)$ a la fracción de fotones emitidos a un ángulo $\beta_{i}$ (Figura 3.10) con respecto al eje de la lluvia, el flujo de luz Cherenkov medido será

$$
y_{i}^{C}=\frac{A \epsilon T^{i}}{4 \pi r_{i}^{2}} f_{C}\left(\beta_{i}\right) Y_{i}^{C} N_{e}\left(X_{i}\right)
$$

Pese a que los fotones Cherenkov son emitidos en un estrecho cono a lo largo de la dirección de las partículas que los generan, cubren un considerable rango angular con respecto al eje de la lluvia. Esto es debido a que la partículas cargadas son desviadas de la dirección del primario debido a dispersión múltiple. Este cono de luz de Cherenkov puede sufrir también dispersión y alcanzar a su vez al detector. En definitiva, puede suceder que una fracción $f_{s}\left(\beta_{i}\right)$ de este cono es dipersada y reulta percibida por el detector, contribuyendo significativamente al flujo total de luz recibida. 
Una simple aproximación para estimar la luz resultante de este fenómeno de dispersión de luz Cherenkov, puede hacerse considerando el número de fotones producidos por el haz a una profundidad $X_{i}$ como la suma de luz Cherenkov producida en las profundidades anteriores $X_{j}$ atenuada por un factor $\tau_{j i}$. Este número se expresa entonces como

$$
N_{\gamma}^{C d i s p}\left(X_{i}\right)=\sum_{j=0}^{i} \tau_{j i} Y_{j}^{C} N_{e}\left(X_{j}\right)
$$

Tenemos entonces que el flujo de luz percibida en los detectores de fluorescencia posee tres orígenes: la luz proveniente de la desexcitación del Nitrógeno $y_{i}^{f}$, la proveniente de directamente de efecto Cherenkov $y_{i}^{C}$ y la correspondiente también a efecto Cherenkov pero habiendo sufrido dispersión previa $y_{i}^{\text {Cdisp }}$. Por lo cual diremos el flujo total de luz es

$$
y_{i}=y_{i}^{f}+y_{i}^{C}+y_{i}^{C d i s p}
$$

Para obtener la energía depositada en la atmósfera se considera la energía cedida por electrones $\left(\frac{d E}{d X_{i}}\right)$ relacionada a su vez con el número de electrones medidos $N_{e}\left(X_{i}\right)$ mediante

$$
\frac{d E}{d X_{i}}=N_{e}\left(X_{i}\right) \int_{0}^{\infty} f_{e}\left(E, X_{i}\right) \frac{d E}{d X_{e}\left(E, X_{i}\right)} d E
$$

donde $f_{e}\left(E, X_{i}\right)$ representa la distribución normalizada de la energía y $\frac{d E}{d X_{e}\left(E, X_{i}\right)}$ es la pérdida de un electrón con energía $E$. La distribución de la energía $f_{e}\left(E, X_{i}\right)$ no depende ni de la energía del primario ni de su composición y por lo tanto la expresión anterior puede simplificarse a

$$
\frac{d E}{d X_{i}}=N_{e}\left(X_{i}\right) \alpha_{i}
$$

donde $\alpha_{i}$ es la energía media depositada por electrón cuando la edad de la lluvia está dada por $s_{i}=3 /\left(1+2 X_{\max } / X_{i}\right)$ donde $X_{\max }$ es el máximo de la lluvia.

En general los detectores de fluorescencia, debido al campo de apertura que poseen, no son capaces de observar completamente el desarrollo total de la lluvia. Dado que para el cálculo de la contribución de Cherenkov y la energía se requiere el perfil completo, la extrapolaciń a las profundidades fuera del campo de visión se hace con una función del tipo Gaisser-Hillas, que da una excelente descripción de los datos de fluorescencia existentes. La función de Gaiser-Hillas tiene la forma

$$
f_{G H}(X)=\frac{d E}{d X_{\max }}\left(\frac{X-X_{0}}{X_{\max }-X_{0}}\right)^{\frac{\left(X_{\max }-X_{0}\right)}{\lambda}} e^{\frac{\left(X_{\max }-X\right)}{\lambda}} \quad\left(X>X_{0}\right),
$$


donde $X_{\max }$ es la profundidad a la cual la lluvia deposita el máximo de energía $d E / d X_{\max }$. La fracción de energía inicial que adquieren las partículas de la componente electromagnética de la cascada está dada por

$$
E_{e m}=\int_{0}^{\infty} f_{G H}(X) d X
$$

Si bien la mayor parte de la energía del primario se deriva en la vertiente electromagnética, definitivamente otras partículas generadas deben tenerse en cuenta en el momento de inferir el valor de la energía total. Se aplica entonces un factor de corrección $f_{\text {inv }}$ que depende de la energía, obtenido a partir de simulaciones. La energía total del primario es entonces

$$
E_{\text {primario }}=f_{\text {inv }} E_{\text {em }}
$$

A modo de resumen diremos entonces que la energía, mediante detectores FD, se obtiene a partir de relacionar la señal en los píxeles con el número de fotones, a su vez considerando que provienen de las desexcitaciones moleculares de $\mathrm{Ni}$, y del efecto Cherenkov. Con estas medidas, y utilizando la función de Gaiser-Hillas, se estima la energía de la componente electromagnética y a través de un factor de proporcionalidad se calcula la energía del primario. 


\section{Capítulo 4}

\section{El Observatorio Pierre Auger}

Esta Tesis Doctoral está realizada dentro del marco del Observatorio Pierre Auger, lo que permitió el acceso a la base de datos, el software oficial, y notas y resultados. Es relevante entonces conocer las características del Observatorio Pierre Auger, de manera tal de comprender mejor el trabajo realizado y los resultados obtenidos.

\subsection{Características generales del Observato- rio Auger}

El Observatorio Pierre Auger [22], [53], [54] fue concebido para medir el flujo, la distribución en la dirección de arribo, y la composición de masa de rayos cósmicos de ultra-alta energía. Fue diseñado para operar en el rango de energías mayores a $10^{18} \mathrm{eV}$, colectar una gran estadística de datos de esas lluvias, y poder tener también una exposición a todo el cielo.

Se encuentra ubicado en las cercanías de la ciudad de Malargüe en Mendoza, Argentina; comenzó a construirse en el año 2000 y desde 2008 funciona completamente. La elección de este sitio estuvo ligada a la altura sobre el nivel del mar (entre $500 \mathrm{~m}$ y $1500 \mathrm{~m}$ ) para observar la lluvia después del máximo desarrollo, características de cielo límpido y con escaso promedio de nubosidad, y ubicación entre los $40^{\circ}$ y $75^{\circ}$ de latitud Sur. Su diseño cubre un área de $3000 \mathrm{~km}^{2}$ de detección (Figura 4.1) utilizando 1600 detectores de superficie y 4 detectores de fluorescencia.

El sello distintivo del Observatorio Pierre Auger es su capacidad, única entre todos los observatorios de rayos cósmicos construidos hasta el presente, de trabajar en un modo de detección híbrido. Hablamos de la naturaleza híbrida de la detección debido a la observación simultánea de las lluvias mediante el uso de los dos tipos de detectores mencionados : arreglo de detectores de 


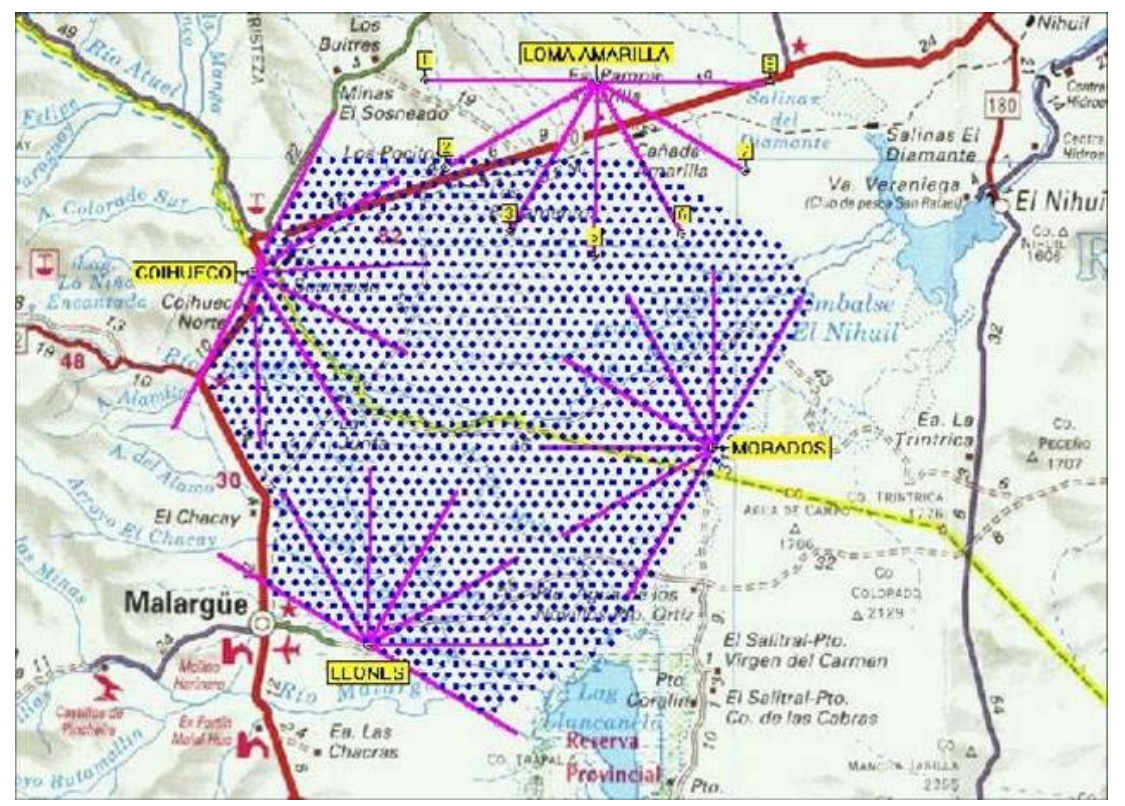

Figura 4.1: Distribución del arreglo de superficie del Observatorio Pierre Auger Sur en la Provincia de Mendoza, Argentina. Los lugares identificados como Loma Amarilla, Los Leones, Los Morados y Coihueco corresponden a los sitios donde se emplazan los detectores de fluorescencia.

superficie y detectores de fluorescencia.

El método de deteccion híbrido permite, como se desprende de las secciones 3.3 y 3.4, conocer con mayor facilidad y precisión los paraámetros más relevantes de las lluvias. El diseño del sitio sur del Observatorio contempla un polígono que abarca el área total de detección en el que se encuentran diseminados de manera regular los tanques de SD. A su vez, sobre pequeños cerros o lomas situados en el perímetro del polígono, y aproximadamente equiespaciados entre sí, se encuentran los cuatro edificios de fluorescencia que contienen 6 cámaras cada uno. Esta distribución de los arreglos de superficie y de fluorescencia permite que la mayor parte de las cascadas detectadas por el FD sean a la vez detectadas por el SD,

Una referencia importante es que los fotomultiplicadores de los detectores de fluorescencia están optimizados para operar con baja intensidad de luz y cubren el rango de longitud de onda entre $300 \mathrm{~nm}$ - $400 \mathrm{~nm}$. Esto conlleva a que el arreglo de FD sólo puede ser utilizado en noches claras de baja actividad lunar por lo que tiene un ciclo útil de sólo el $10 \%$, aunque sus datos pueden utilizarse para calibrar la escala de energía del SD. Por otro lado, los detectores de superficies no sufren ese problema, por lo que su ciclo 
de utilidad es del $100 \%$ del tiempo.

\section{2. $\quad$ El arreglo de detectores de superficie}

Los detectores de superficie registran la llegada al suelo de las partículas secundarias de la lluvia. Debido a que la probabilidad de detectar una lluvia generada por un primario de $100 \mathrm{EeV}$ es del orden 1 por siglo por $\mathrm{km}^{2}$ (Capítulo 1), y a que la cantidad de partículas secundarias y a su dispersión en este rango de energías, es necesario un arreglo de detectores que cubra una gran extensión. En el caso del Observatorio Auger las estaciones de detección son estaciones Cherenkov de agua espaciados por $1.5 \mathrm{~km}$ en una grilla regular sobre el terreno.

La forma de los tanques es cilíndrica, con una superficie superior de $10 \mathrm{~m}^{2}$ y una altura de $1.2 \mathrm{~m}$ (Figuras 4.2, 4.3). En ese volumen disponible se sitúa una bolsa de tres capas (denominada liner); y recubierta en su parte interior de Tyvek plástico negro, un material óptimo para la reflexión de fotones, que se llena con $12 \mathrm{~m}^{3}$ de agua ultra-pura. En ese medio es donde se produce la luz de radiación Cherenkov. Se utiliza agua ultra-pura de manera tal que se mantengan sus propiedades durante el tiempo de vida del experimento, estimado en 20 años.

Para colectar la luz Cherenkov se utilizan tres fotomultiplicadores XP1805 Photonis de $24 \mathrm{~cm}$ de diámetro aproximado y forma semiesférica. Estos fotomultiplicadores están instalados en la tapa superior y mirando hacia adentro del tanque. Están apoyados sobre tres ventanas de polietileno traslúcido que se encuentran en la bolsa que contiene el agua ultra-pura, y a partir de los cuales pueden recibir los fotones Cherenkov. A estos fotomultiplicadores los denominaremos sPMT para indicar su uso en los detectores de superficie y diferenciarlos de los fPMT utilizados en los detectores de fluorescencia.

Las señales en cada PMT son tomadas del ánodo y del ultimo dínodo. La señal del último dínodo es amplificado por un factor 32, extendiendo de esta manera el rango dinámico y permitiendo ampliar la medida de unos pocos fotoelectrones hasta aproximadamente $10^{5}$.

La señal ánodo-dínodo se filtra y digitaliza a $40 \mathrm{Mhz}$ usando un Conversor rápido análogo digital (Flash Analog Digital Converters FADCs) que además determina la línea base de la señ al y sus posibles fluctuaciones.

El tanque tiene además una antena de comunicaciones para enviar los datos colectados a la estación central de colección de datos, una antena de posicionamiento global satelital (GPS), una caja que protege la electrónica de los detectores y la de la comunicación; y un pequeño panel solar y una batería de $12 \mathrm{~V}$ que son quienes combinadamente proveen $10 \mathrm{~W}$ de potencia 


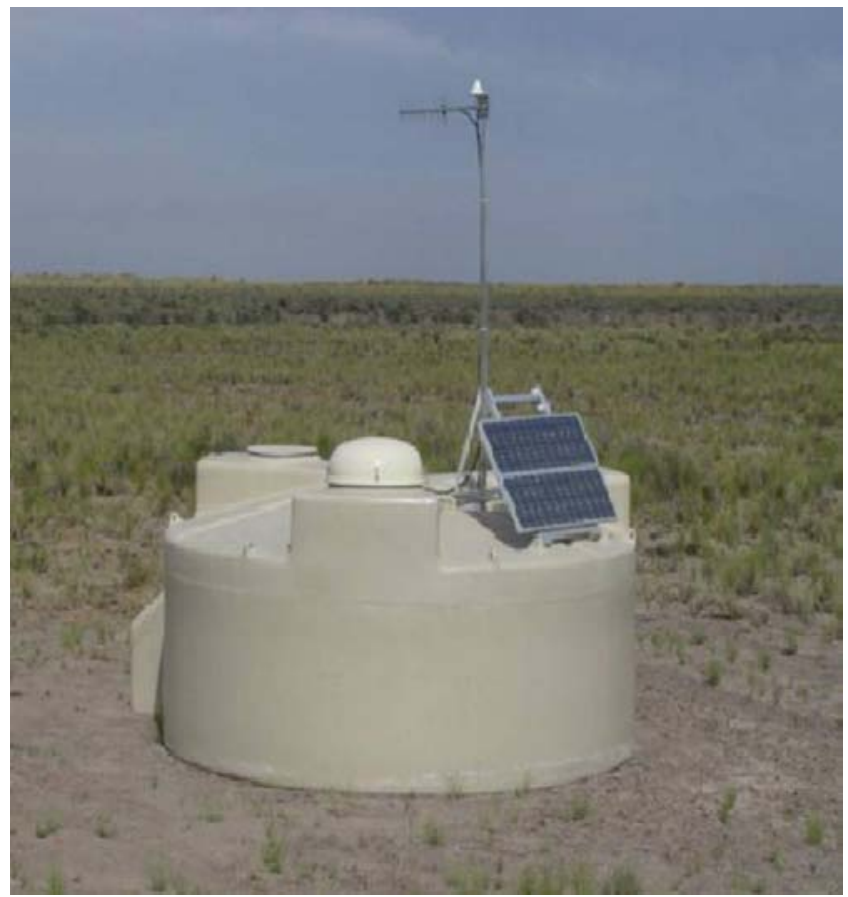

Figura 4.2: Imagen de uno de los detectores Cherenkov de superficie.

para el normal funcionamiento.

\subsubsection{Calibración de los tanques Cherenkov}

El proceso de calibración es obviamente imprescindible en todos los experimentos. La particularidad en los experimentos de esta envergadura, es que por la gran cantidad de detectores y su dispersa ubicación, debe hacerse de forma automática en cada detector. Además debe poder realizarse independientemente de que todos los PMT estén en funcionamiento. La calibración debe considerar que la señal producida en una estación depende de varios parámetros: la pureza del agua, la reflectividad en el liner, la ganancia de los PMT, la ganancia del último dínodo y la ganancia dínodo-ánodo amplificada.

Como ya fue establecido en el capítulo anterior, la señal en los detectores se expresa en VEM (Vertical Equivalent Muon). La distribución de luz producida por estos muones en el liner produce una señal caracterizada por un pico inicial producto del algoritmo de disparo y un pico secundario relacionada al VEM (Figura 4.4). Estrictamente, la suma de las cargas en todos los fotomultiplicadores corresponde al total depositado en el tanque, mientras que sPMT individuales son sensibles solo a una fracción de la señal. De 


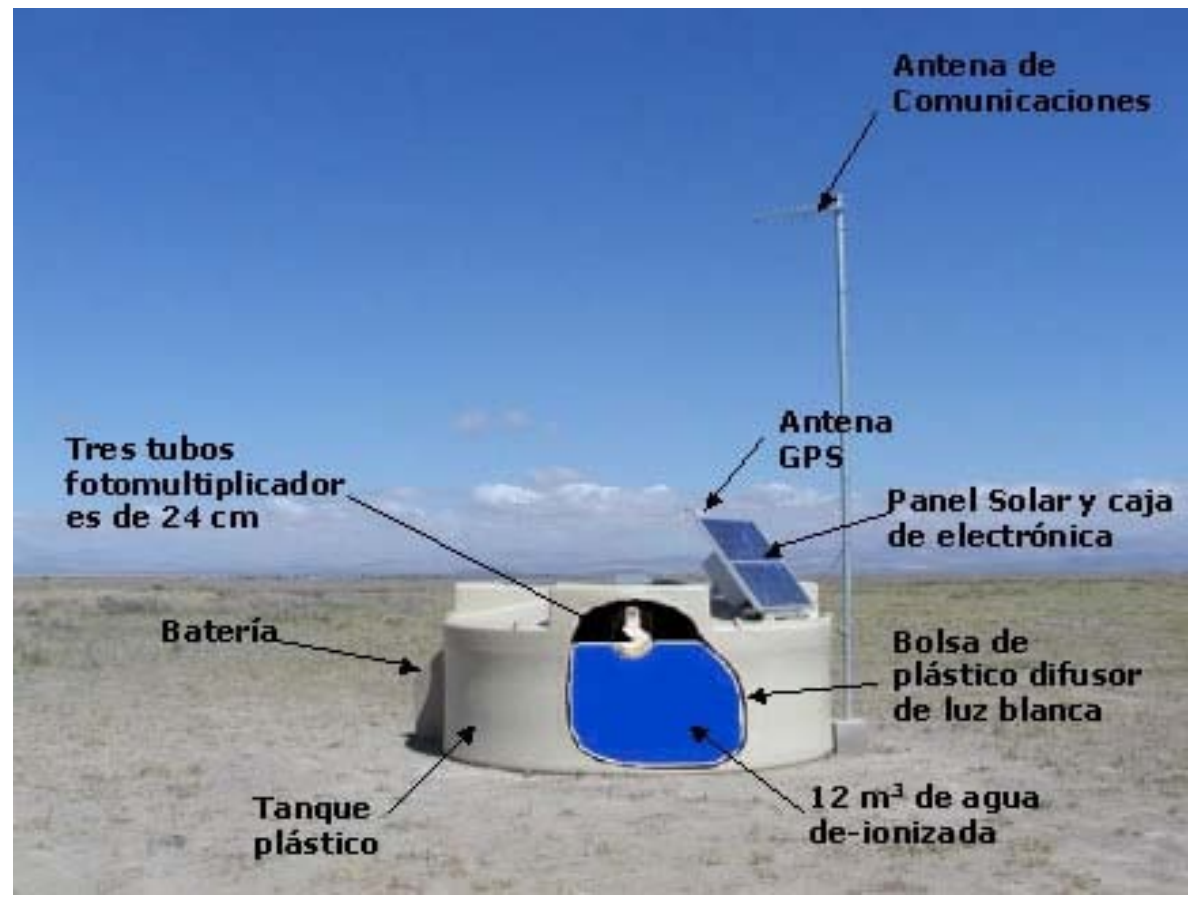

Figura 4.3: Diagrama de un detector de superficie y sus componentes.

modo que, si la señal fue medida por todos los PMT, el pico corresponde aproximadamente a $1.09 \mathrm{VEM}$, mientras que resulta $1.03 \pm 3 \mathrm{VEM}$ para PMT individuales. Para determinar con precisión la llegada de muones verticales se utilizan centelleadores correlacionados en la parte superior e inferior de un tanque de referencia.

Considerando además que el flujo de muones atmosféricos es muy abundante y prácticamente continuo, la calibración puede realizarse en cualquier momento. Aprovechando esta posibilidad, los tanques SD se calibran una vez por minuto para monitorear las fluctuaciones de la respuesta de la estación con el tiempo o las fluctuaciones de la respuesta entre estaciones.

\subsection{2. $\quad$ Trigger y Adquisición de Datos}

La digitalización de las seis señales (dos por sPMT) de una estación SD a 40 Mhz y 10 bits genera cerca de 300 MB por segundo de información. Si todos los detectores generan señal simultáneamente, el caudal de datos a procesar resulta ser de 4.7 GB por segundo. Sin embargo la mayoría de estos datos corresponden principalmente a muones atmosféricos y cascadas de muy baja energía, todos eventos que no resultan de interés dentro del 

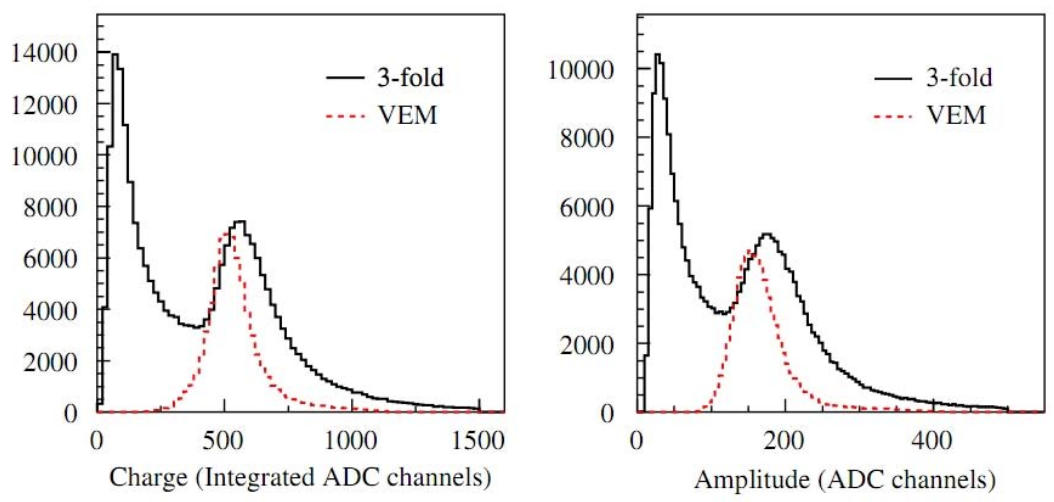

Figura 4.4: Histogramas de carga y altura del pulso de un tanque Cherenkov con medida en 3 sPMT (negro). El histograma en rojo corresponde a la señal de muones verticales determinando la escala en VEM. La posición del máximo secundario determina el valor del parámetro $I_{V E M}$ utilizado para ajustar los trigger T1 y T2 [73].

Observatorio Pierre Auger.

Como el objetivo es estudiar las lluvias superiores a los $10^{18} \mathrm{eV}$, el sistema de trigger fue diseñado para colectar datos de las estaciones cuando estamos en presencia de una de estas lluvias. Este mecanismo puede ser pensado como una cadena de varios niveles, comenzando en una simple selección de señales en cada tanque (triggers T1 y T2), luego otra selección acorde a lo que midieron los otros detectores SD del arreglo (nivel de trigger T3) y por último una selección de señales que encuentran correlato con eventos de rayos cósmicos (trigger T4 y T5). Es importante destacar que los niveles T1, T2 y T3 se realizan inmediatamente generada la señal, mientras T4 y T5 se realizan a posteriori.

El trigger $\mathbf{T} 1$ posee dos modos:

- ToT ( Time over Threshold, tiempo sobre el umbral) permite identificar que la señal en un tanque tenga posibilidad de pertenecer a una lluvia real. Consiste en que al menos $2 \mathrm{sPMT}$ superen los $0,2 I_{V E M}$ (el valor correspondiente a la amplitud de la señal de $1 \mathrm{VEM}$ ) en al menos 13 intervalos temporales en un intervalo de $2 \mu$ s (120 bines de tiempo) (Figura 4.5.a).

- ToS (Signal over Threshold, señal sobre el umbral) es un algoritmo no muy eficiente, con una frecuencia de disparo de $100 \mathrm{~Hz}$, pero es imprescindible para identificar señales de corto tiempo, propias de la 
componente muónica de cascadas muy inclinadas. El algoritmo consiste en que la señal en los tres sPMTs supere 1,75 $I_{V E M}$ por encima de la línea de base en al menos un intervalo temporal.

El segundo nivel de trigger T2, al igual que T1 desarrollado por el software del tanque, tiene también dos modos:

- Thr2 (Threshold trigger, disparador de umbral) requiere una coincidencia de 3 sPMT por encima de 3.2 VEM. Esto es necesario para señales extendidas en el tiempo, producidas por lluvias muy energéticas con centro cercano al detector o la componente muónica en lluvias horizontales. Las trazas de un detector que supera el Thr2 se encuentra en la Figura 4.5.b

- ToT2 (Time over threshold) implica una coincidencia de al menos 2 sPMT con señales que incluyan no menos de 13 bins por encima de $0.2 \mathrm{VEM}$ dentro de una ventana de tiempo de $3 \mu$ s. Esto selecciona pequeñas señales producidas por lluvias con centro lejos del del detector o lluvias de baja energía. Un ejemplo de señales de estaciones que alcanzan este nivel se muestran en Figura 4.5.c .

Cuando la señal satisface los triggers Thr2 y ToT2, sólo el segundo es observado. si un tanque satisface T2 entonces automáticamente satisface la condición T1. Si la señal supera los parámetros fijados para el trigger T2, se envía a una de las antenas colectoras que están ubicadas estratégicamente en la periferia del arreglo. Estas antenas envían luego las señales de los detectores al Centro de Adquisición de Datos (Central Data Aquisition System - CDAS) continuar la depuración y seleccionar para el análisis.

El nivel de trigger T3 se implementa en el CDAS y corresponde a correlacionar la señal de un detector con la de sus vecinos, obviamente todos alcanzaron el nivel T2. Toma una ventana de coincidencia temporal de 50 $\mu$ s entre todas señales y busca correlaciones espaciales entre ellas. Existen también dos modos:

- El primer modo se aplica a los ToT2 y requiere que al menos dos estaciones vecinas alcancen ToT2. De ellas, al menos una debe estar entre uno de los seis primeros vecinos a la estación en la que se está evaluando el algoritmo y la restante debe estar entre los primeros o los segundos vecinos. Este modo tiene una eficiencia del $90 \%$ en la determinación de eventos correspondientes a lluvias reales.

- El segundo modo se aplica a ambos tipos de T2 y es menos riguroso en términos de la posición relativa de los tanques con señal. Requiere 

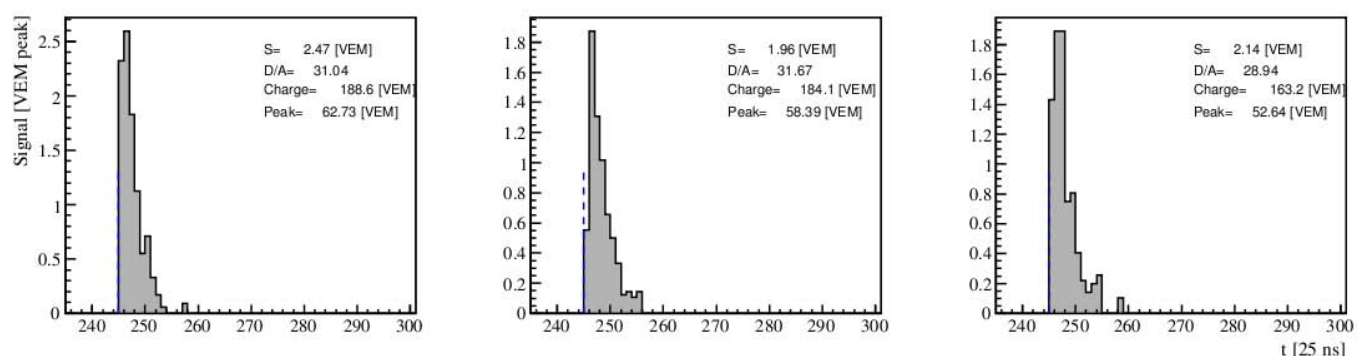

(a) Señal en un detector que alcanza el trigger T1.
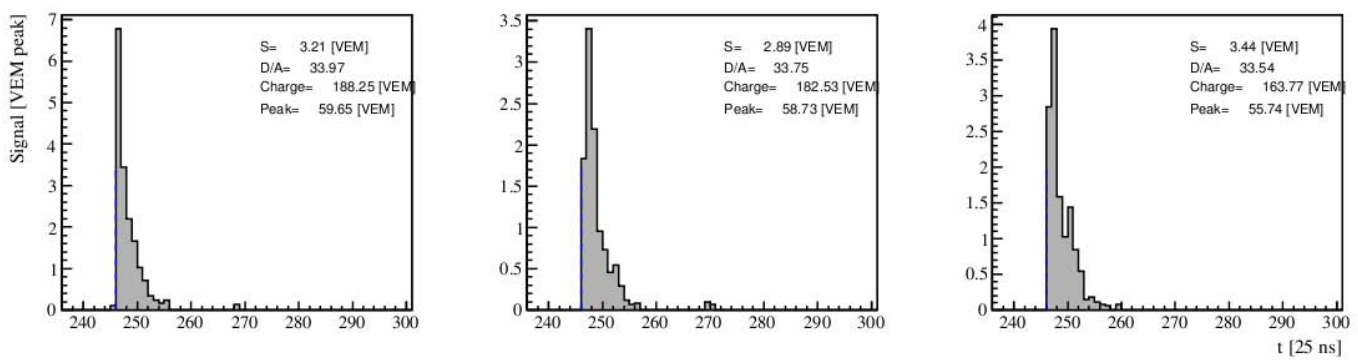

(b) Señal en un detector que alcanza el trigger T2.
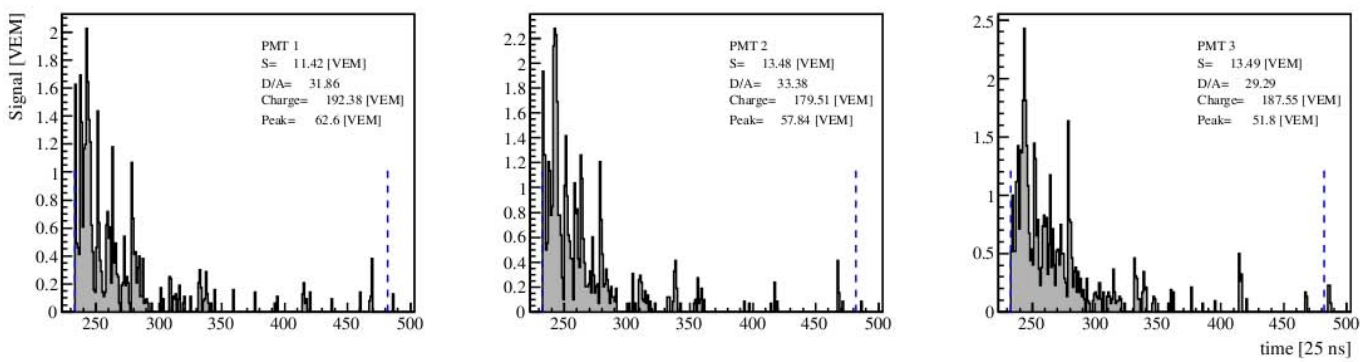

(c) Señal en un detector que alcanza el trigger ToT2.

Figura 4.5: Señales en detectores que alcanzan los niveles de trigger T1, T2 y ToT2. 
tres tanques como mínimo con T2, uno entre los primeros vecinos y los restantes entre los primeros o los segundos; y el cuarto en cualquiera de los primeros, segundos, terceros o cuartos vecinos. Este modo posee una eficiencia muy baja $(2 \%)$ ya que no elimina a las coincidencias accidentales, pero sin embargo es necesario para no eliminar a las cascadas inclinadas.

Una vez que se confirma la compatibilidad espacial entre detectores vecinos acorde a una lluvia, se aplica el trigger T4. Éste es un nuevo algoritmo que involucra la física propia de las cascadas, ya que elimina los tanques con señales accidentales de muones atmosféricos y resulta el pimer paso en la identificación de lluvias verticales pausibles de ser reconstruidas. Como los anteriores, T4 cuenta con dos modos:

- Requiere al menos 3 tanques con T3 alcanzado, situados en una configuración espacial no alineada. Es una condición que selecciona efizcamente el $99 \%$ de eventos con inclinación menor a $60^{\circ}$ [74][75] pero no es muy eficiente para lluvias inclinadas.

- El modo 4C1 es alcanzado sólo por eventos detectados por 4 tanques en un nivel T2 y una configuración espacial de un tanque con 3 primeros vecinos (Fig 4.6).

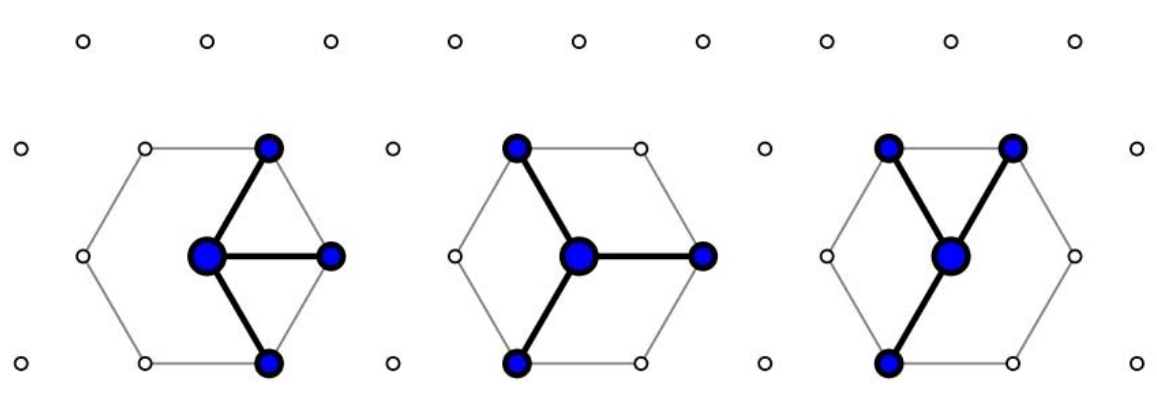

Figura 4.6: Posibles configuraciones espaciales necesarias para el trigger T4.

En todos los casos de tanques que alcanzan el nivel T4 se requiere una condición temporal adicional: la diferencia entre los tiempo de inicio de la señales debe ser menor que la distancia entre ellas dividida por la velocidad de la luz, que resulta equivalente a una límite de 200 ns. Esto corresponde a la física propia de frentes de lluvia. 
Por último se encuentra el nivel T5 [76], asociado a un corte de calidad del evento. Consiste en que la estación de mayor señal tenga los 6 primeros vecinos en funcionamiento (no necesariamente todos con señal) en el momento de la medida (Figura 4.7).

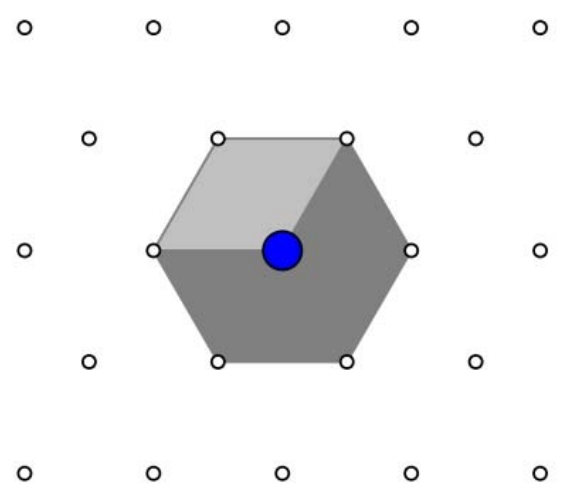

Figura 4.7: Configuración espacial necesaria para el trigger T5.

Permite excluir aquellos eventos medidos en el límite del arreglo SD. Esta clase de eventos, debido a posibles pérdidas de señales se excluyen de los seleccionados para reconstrucción. Los eventos que alcanzan el nivel T5 son los considerados para el análisis. Los eventos que además fueron detectados y reconstruidos por el detector de fluorescencia se denominan eventos híbridos dorados, y son los utilizados para la calibración de energía del arreglo SD.

\subsubsection{Algunos comentarios adicionales sobre los tan- ques SD}

Como ya fue planteado previamente, la señal que registran los detectores de superficie representa la intensidad de la señal medida en VEM en función del tiempo. Un ejemplo de las señales obtenidas en los detectores SD ante un evento puede observarse en Figura 4.8. Para una misma lluvia la diferencia en las señales dependerá de la distancia al centro de la lluvia, el ángulo de inclinación y la dirección azimutal de donde provino la lluvia. En la Figura 4.9 se muestra la ubicación de las estaciones que tuvieron señal en el evento 184599, proyectadas en el plano de la lluvia. El tamaño de los círculos es proporcional al logaritmo de la señal en VEMs. 

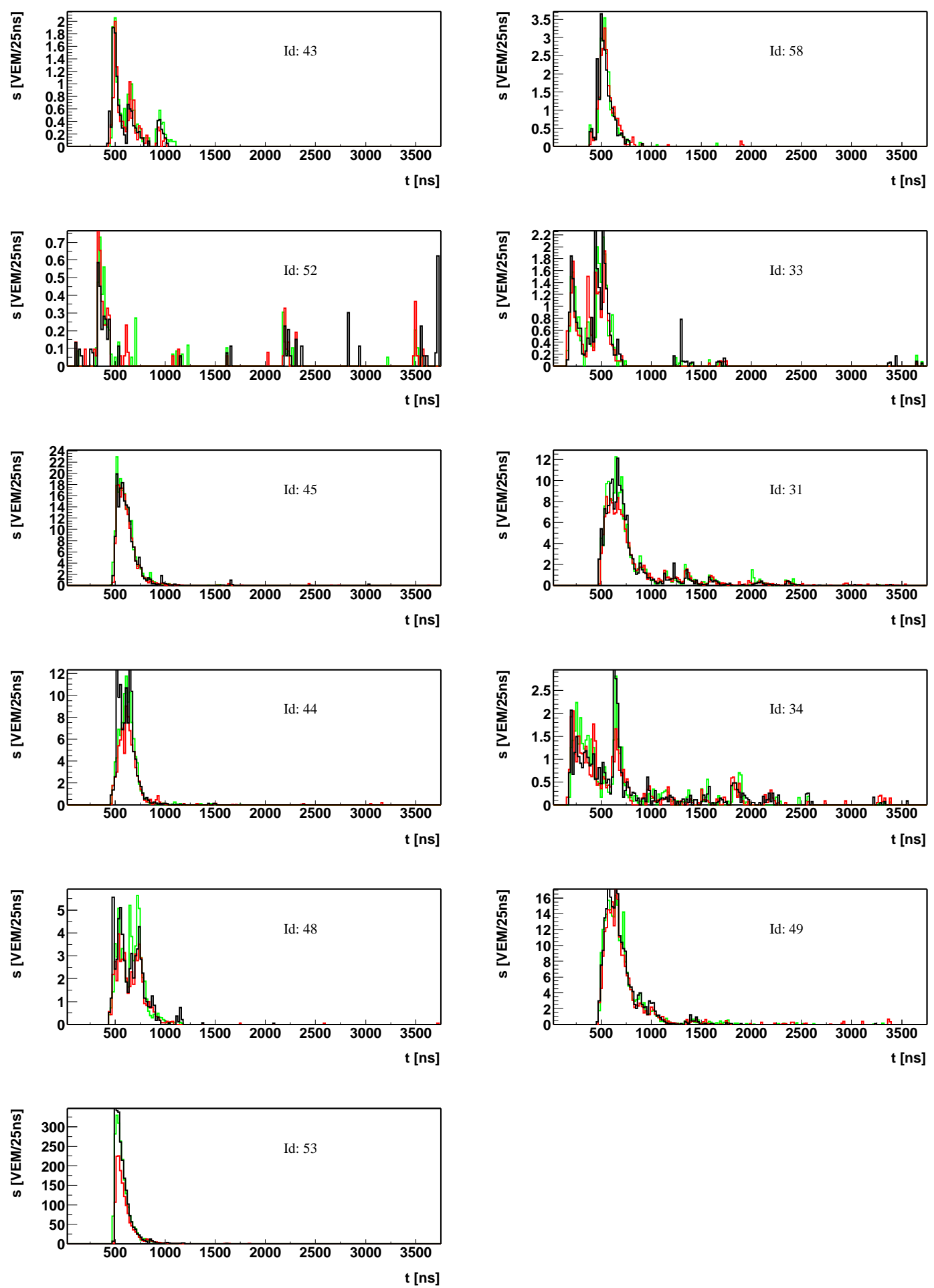

Figura 4.8: Señales de los detectores de superficie correspondientes al evento 184599. 


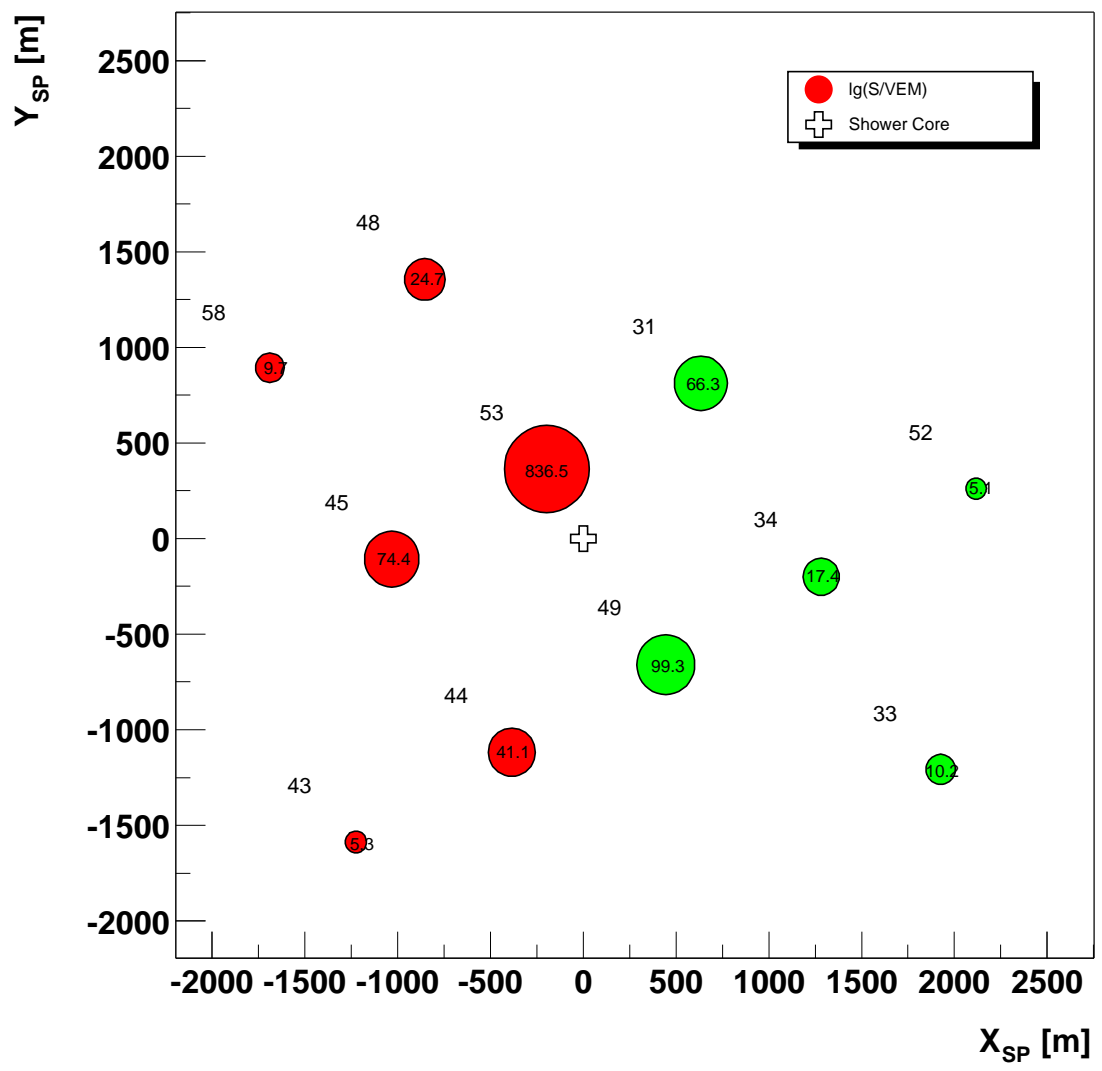

Figura 4.9: Diagrama explicativo sobre el evento 184599 detectado por el arreglo de superficie. El tamaño de los círculos representa la intensidad de la señal registrada en el correspondiente detector.

Los fotomultiplicadores tienen un sistema de calibración previo a la instalación del tanque en el arreglo, realizado en el Campus Central del Observatorio. Esta calibración está referida a la sincronía en el voltaje umbral, la evolución en la toma de la señal y la comparación en la secuencia de medidas con un tanque modelo.

La calibración temporal de los detectores se realiza con el sistema de GPS. Estudios de la precisión en las medidas temporales fueron realizadas con tres pares de detectores (Carmen-Miranda, Moulin-Rouge, Día-Noche). La distancia entre los tanques de cada par es de $L=11 \mathrm{~m}$. Debido a esta escasa diferencia $(\Delta t=L / c)$, ambos detectores comienzan a recibir las partículas practicamente en el mismo instante. La mayoría de los triggers de estos pares 
se deben a lluvias de menor energía que, como son más frecuentes, permiten una gran colección permanente de datos para este estudio.

Con estos pares de detectores se determinan las incertezas en las medidas de las señales y sus distribuciones temporales. Estudios realizado en el Observatorio Auger [55] determinaron la incerteza de la señal medida, es decir el $\sigma$ necesario en la ecuación (4.11). El valor obtenido fue

$$
\begin{gathered}
\sigma=[1.06 \pm 0.05] \times \sqrt{S} \\
\sigma(\theta)=[0.66+0.2 \sec (\theta)] \times \sqrt{S}
\end{gathered}
$$

donde $S$ representa la señal del detector medida en VEM. La primera expresión promedia sobre todos los ángulos zenitales y el segundo explicita esa dependencia.

\subsection{Detectores de fluorescencia}

Para completar la breve descripción del Observatorio Auger desarrollaremos las propiedades principales de los detectores de fluorescencia. Por la características híbridas del Observatorio, los detectores de fluorescencia funcionan siempre en sincronía con el arreglo de detectores de superficie. En esta constructiva conjunción de mediciones acopladas, la técnica de fluorescencia es quien provee la mejor manera de medir energía de la partícula primaria. Como recordamos del capítulo anterior, esta medición se logra por el hecho de que la cantidad de luz de fluorescencia producida por la interacción de las partículas de la lluvia con las moléculas de la atmósfera es proporcional al número de partículas cargadas, permitiendo una medida directa del desarrollo longitudinal de la lluvia en la atmósfera. El cálculo de la energía del primario se realiza integrando el perfil de lluvia medido y agregándole un pequeño factor de corrección (entre el $8 \%$ - 13\%) debido a la componente de la cascada que no puede ser detectada por este dispositivo (muones y neutrinos fundamentalmente).

El arreglo de detectores de fluorescencia que posee el sitio sur del Observatorio Auger consiste en 4 "ojos de fluorescencia" localizados en la periferia del arreglo de superficie con el objetivo de observar el desarrollo de lluvias en el volúmen de atmósfera sobre el mismo (Figura 4.1). Cada detector de fluorescencia está situado en un edificio particular sobre una elevación del terreno y comprende seis telescopios, cada uno orientado de manera de cubrir $180^{\circ}$ en azimut. Todos los ojos de fluorescencia se encuentran activos y son nombrados de acuerdo al sitio donde se encuentran instalados; "Los Leones", "Coihueco", "Los Morados" y "Loma Amarilla" (Figura 4.10). Con esta configuración cada telescopio cubre $30^{\circ}$ azimutales y $28.6^{\circ}$ de elevación. 


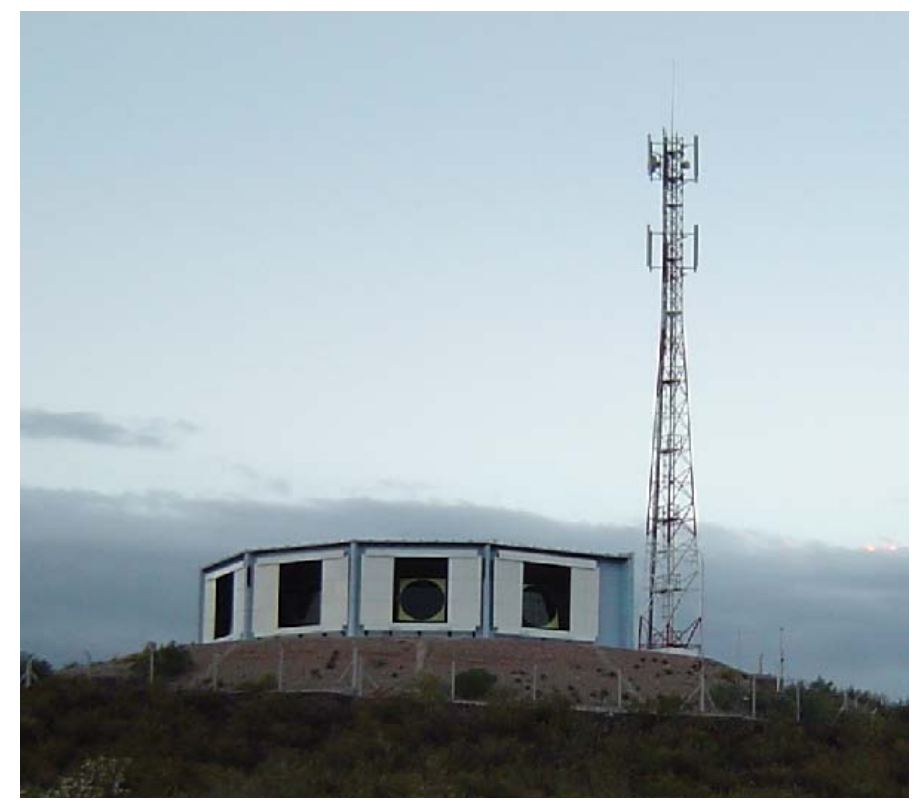

Figura 4.10: Edificio de "Los Leones". Se observa la torre de comunicaciones y las cortinas metálicas abiertas exponiendo el anillo y el filtro.

Los telescopios son protegidos de la luz solar, la lluvia, el viento y el polvo con unas cortinas metálicas llamadas shutter que se encuentran sobre las paredes exteriores del edificio, justo delante de cada telescopio. Estas cortinas se desplazan en el momento de cada medida vía remota en los momentos establecidos para la adquisición de datos. Están conectados además con los higrómetros y anemómetros situados en la estación climática de cada uno de los edificios, de manera tal que se cierran automáticamente ante lluvias o fuerte viento.

El sistema óptico de los telescopios está diseñado de acuerdo a la configuración de Schmidt (Figura 4.11), consistente en una apertura determinada por un diafragma circular centrado en el centro de curvatura de un espejo esférico y con la cámara de fotomultiplicadores en su foco. Esta configuración disminuye la aberración óptica.

La apertura de cada telescopio es de 2,2 m de diámetro y cuenta con un anillo corrector de radio interno $0,85 \mathrm{~m}$ y externo $1,1 \mathrm{~m}$ de lentes traslúcidos a las longitudes de onda ultravioletas [77] corrige la aberración esférica introducida por el aumento en la apertura (el diseño original incluía una apertura de $1.7 \mathrm{~m}$ de diámetro). Con el objeto de preservar al telescopio de luz de longitud de onda no deseada e incluso del polvo presente en el aire, el mismo posee un filtro compuesto de láminas de $3,25 \mathrm{~mm}$ de espesor [78]. Este filtro 
bloquea casi totalmente la luz en el rango visible, reduciéndo la luz de ruido de fondo en un factor 8. Por otro lado es transparente a las longitudes de onda entre $300 \mathrm{~nm}$ y $400 \mathrm{~nm}$, propias de la luz de fluorescencia.

Cuando la luz de fluorescencia atraviesa el filtro, se refleja en otro de los componentes del telescopio: el espejo esférico de $3,4 \mathrm{~m}$ de curvatura y de dimensiones $3,8 \mathrm{~m} \times 3,8 \mathrm{~m}$. El mismo está constituidos en forma de paneles mas pequeños que permiten un enfoque más sencillo, 36 paneles de aluminio pulido en Los Leones y Los Morados, y 60 segmentos hexagonales de vidrio que conforman un panal en Coihueco y Loma Amarilla. Ambos tipos de espejos poseen una eficiencia del $90 \%$ para fotones en el rango de fluorescencia.

Luego de ser reflejada en el espejo, la luz se dirige al foco del espejo, donde se encuentra una cámara pixelada con 440 fotomultiplicadores de superficie hexagonal en un arreglo de 22 filas por 20 columnas. A estos fotomultiplicadores los denotaremos fPMT haciendo referencia a su uso en los detectores de fluorescencia. Considerando 4 edificios y cada uno de ellos con 6 cámaras, el total es de 10560 fotomultiplicadores en funcionamiento.

Cada píxel corresponde a un fotomultiplicador Photonis XP3062 de 3,6 pulgadas de diámetro y que permite una apertura de $0,75^{\circ}$. En cada intersticio entre fotomultiplicadores se encuentran un plástico reflectante que permite derivar hacia los fotomultiplicadores adyacentes la luz que haya incidido sobre él. De esta manera se logra mayor recepción de la traza de la lluvia.

Cada una de las cámaras posee en la parte baja del soporte, una serie de 20 placas de digitalización (una por columna de fPMT) que mediante un módulo de conversión analógico-digital rápido (FADC) de 10 Mhz y 12 bits, comienzan el procesamiento de la señal FD.

\subsubsection{Trigger}

El volumen de manejo de señales en cada cámara es en sí mismo superior al del arreglo SD, ya que con el equipamiento detallado, el módulo FADC procesa $6,3 \mathrm{~Gb} / \mathrm{s}$. Evidentemente esto exige una depuración inicial de las medidas, de manera de permitir una razonable cantidad de datos de calidad para el análisis. Al igual que con los detectores SD, esto se logra mediante una serie triggers o niveles de trigger. En particular, los detectores de fluorescencia del Observatorio Pierre Auger constan de 3 niveles de trigger.

- First Level Trigger o FLT: es la primera instancia de selección de señales y lo realizan las placas digitalizadoras de las cámaras. Consiste en detectar los fPMT con señal diferente de ruido, proceso que se logra cuando la suma de las señales de las últimas muestras en cada 


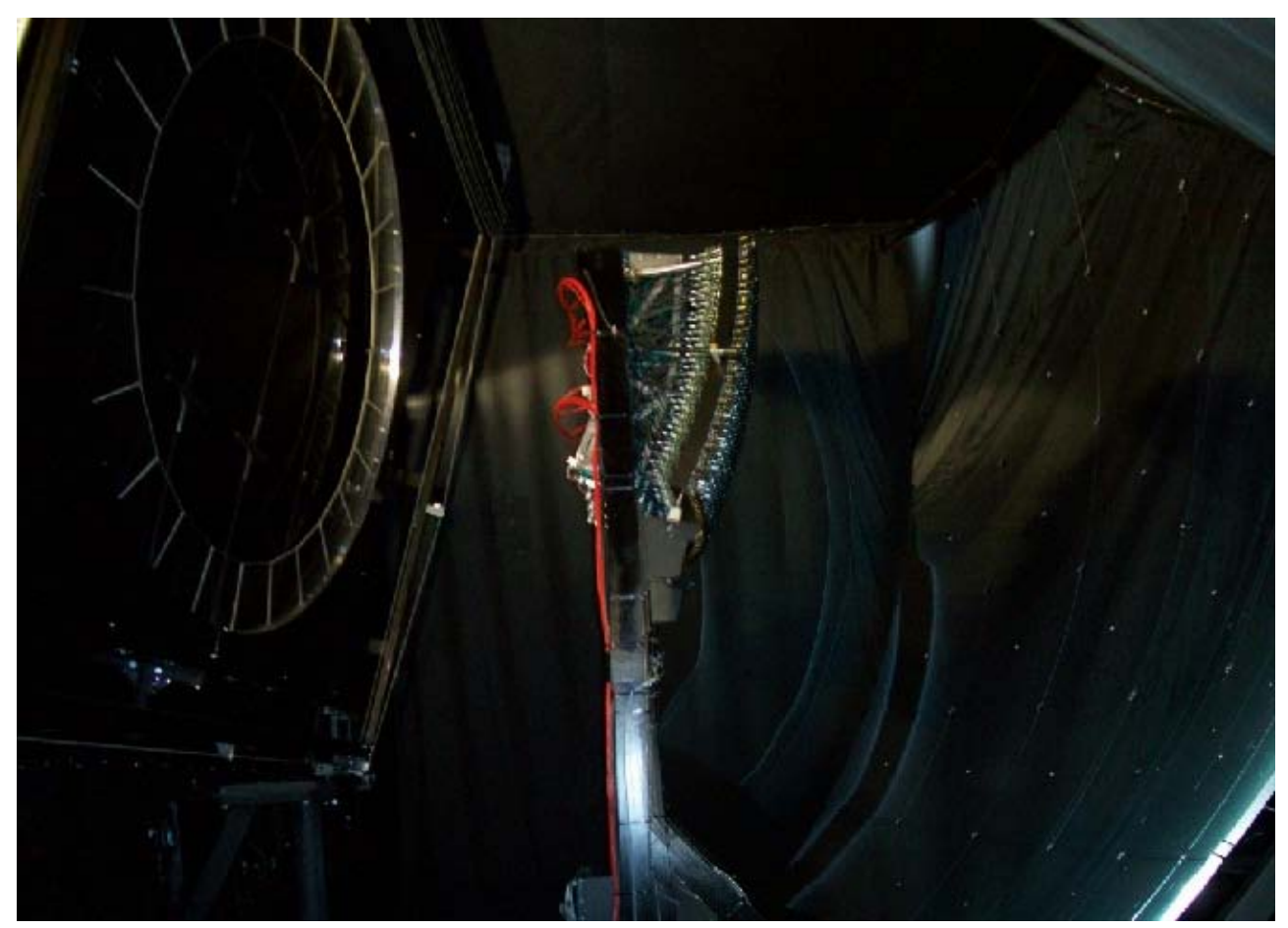

(a) Montaje de uno de los telescopios de fluorescencia. De izquierda a derecha se observa: el anillo corrector con el filtro, la cámara con los fotomultiplicadores y el espejo.
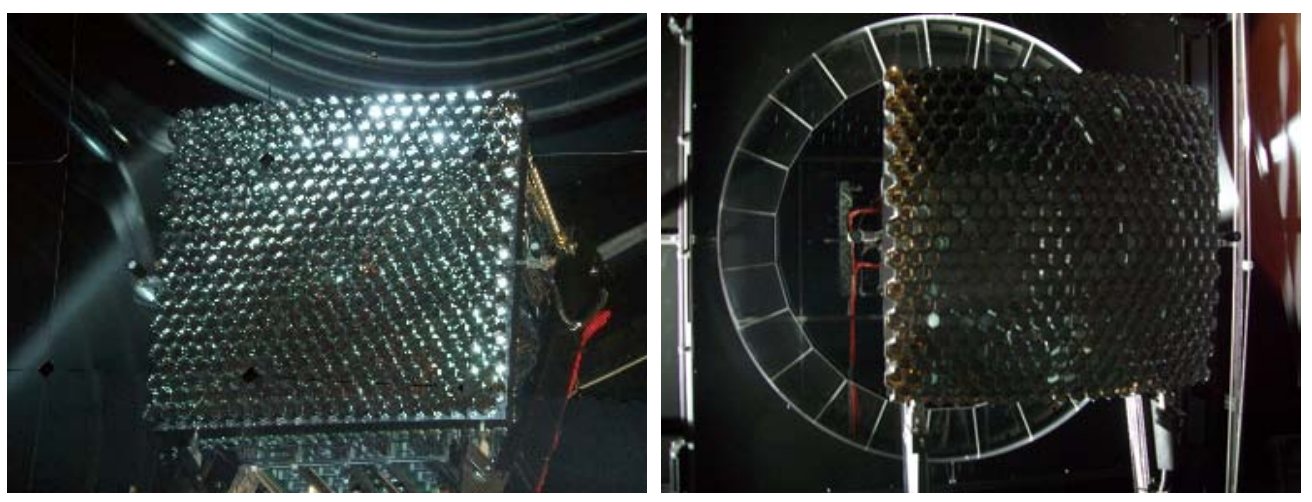

(b) Detalle de la cámara reflejada sobre el (c) Detalle de la cámara y el anillo de enespejo. Nótese en los laterales de la cámara trada con el filtro. las fuentes de calibración B.

Figura 4.11: Equipamiento de uno de los telescopios de fluorescencia. 
píxel supera un cierto umbral. El umbral se ajusta continuamente para mantener la frecuencia del trigger en $100 \mathrm{~Hz}$.

- Second Level Trigger o SLT: se realiza mediante una segunda placa posterior al FADC. Realiza la identificación de una figura similar a la dejada por una posible lluvia, tomando en cuenta a todos los píxeles con señal. Busca que 5 píxeles adyacentes tengan un patrón de coincidencia temporal en una ventana de tiempo de 1 a $32 \mu$ s. Este trigger se realiza con una frecuencia de $0.1 \mathrm{~Hz}$ en cada cámara.

- Third Level Trigger o TLT: corresponde a la última instancia de depuración, y se realiza a través de software en una computadora denominada MirrorPC situada en el edificio del detector. Este software realiza una búsqueda de correlación espacio-temporal entre las señales de los fPMT, seleccionando por sobre imágenes no deseadas. El promedio de este trigger es de $0.02 \mathrm{~Hz}$ por cámara [80].

Cuando un evento alcanza el nivel de trigger TLT, la señal de todos los fPMT que alcanzaron el nivel FLT se conserva para realizar un segundo chequeo y evitar pérdida de medidas valiosas. Esta información se destina a otra computadora $(E y e P C)$ que se encarga además de chequear si el mismo evento ha sido detectado por una de las cámaras contiguas. En el caso de que se produzca, se conservan mediciones en el mismo archivo. Luego toda esta información es enviada al CDAS.

También la EyePC, se realiza una primera reconstrucción de la lluvia con el objetivo de estimar los parámetros principales de la lluvia en relación a su geometría y tiempo de arribo. Esta primera reconstrucción no posee alta precisión y es sólo para poder determinar los eventos válidos, clasificados como T3FD, y que serán aquellos que superan la reconstrucción con más de 4 píxeles.

En el CDAS se correlacionan las medidas FD con las SD para clasificar al evento como híbrido, considerando a todas aquellas estaciones con señal cercanas al core dentro de una ventana de tiempo de $120 \mu \mathrm{s}$.

\subsubsection{Calibración de las cámaras}

Tal compleja medición, sensible además a bajo flujo lumínico requiere un sistemático plan de calibración. Las cámaras poseen dos momentos de calibración, denominados calibración absoluta y calibración relativa.

La calibración absoluta se realiza varias veces al año y consiste en testear la capacidad de respuesta de cada fPMT a un haz de luz conocido. Para este proceso se cubre la superficie del diafragma con un dispositivo conocido 
como drum (por su semejanza a un tambor) que difunde sobre una de las caras la luz de un led optimizado en $375 \mathrm{~nm}$ (Figura 4.12). Esta luz difusa llega luego al espejo y posteriormente a la cámara. La completa calibración incluye además trabajar con otras longitudes de ondas y filtros, siempre en el rango $300-420 \mathrm{~nm}$.
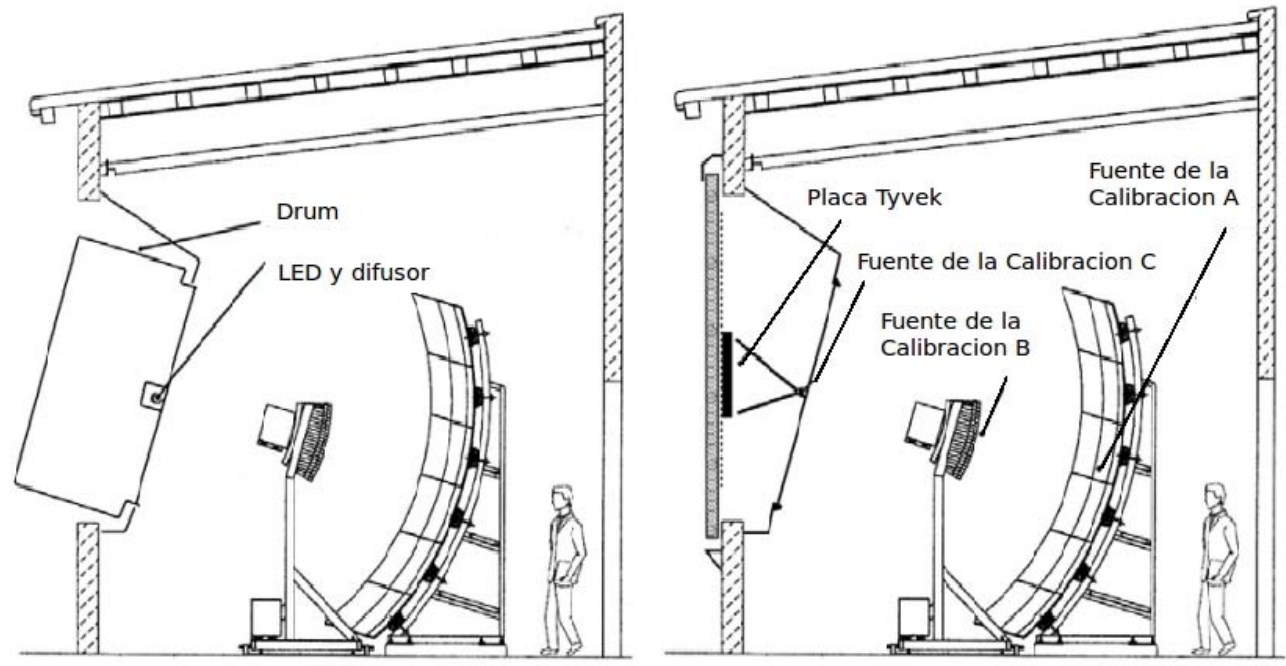

Figura 4.12: Diagrama de la configuración de los dispositivos utilizados en la calibración absoluta (izq) y relativa (der).

La calibración relativa se realiza cada noche del turno de medición de FD en forma remota, tanto al comienzo como al final de la misma y obviamente con las cortinas de protección cerradas. Consiste en tres instancias (A, B y C) que corresponden a la respuesta de la cámara a fuentes en tres lugares diferentes y de longitudes de onda distintas (Figura 4.12). La calibración se realiza secuencialmente - primero $\mathrm{A}$, luego $\mathrm{B}$ y por último $\mathrm{C}$ - pero en forma simultánea sobre todas las cámaras de cada detector. De esta manera se evalúa también la sincronía en las medidas de las cámaras ya que las fuentes que inciden sobre cada cámara se inician en el mismo instante. La diferencia temporal media típica es de 7 ns.

Desarrollemos un poco más las instancias de la calibración relativa:

- Calibración A: responde a una fuente tipo LED de $470 \mathrm{~nm}$ situado en el centro del espejo e iluminando en forma directa a toda la cámara.

- Calibración B: implica flashes de Xenon con longitudes de onda 320, 337, 355, 380 y $455 \mathrm{~nm}$, situados en los laterales de la cámara (Figura 
4.11) que determinan la alineación cámara-espejo.

- Calibración C: también realizada mediante flshes de Xenon pero ubicados sobre el diafragma e incidiendo sobre una placa de Tyvek situada sobre la cara interna de los shutters, que difunde y refleja la luz hacia el espejo. De este modo se evalúa el funcionamiento del filtro.

Además de tomarse en cuenta las calibraciones relativas para las estimaciones de cada medida; se efectúa un chequeo cruzado de las informaciones correspondientes a la calibración relativa y a la absoluta. Se considera normal una variación en la respuesta del detector noche a noche de entre $3 \%$ y $5 \%$ [79]. Esto incrementa el error total en la calibración a un 9,5\%.

\subsection{Otros detectores en desarrollo}

El Observatorio Pierre Auger continúa incorporando detectores específicos de menor dimensión, pero de importante complemento con los arreglos SD y FD.

El mayor ejemplo de ello lo constituye el Auger Engineering Radio Array (AERA) [85] que, como su nombre lo indica, es un detector de radio (RD). Los detectores de radio miden, mediante el uso de antenas, la emisión de radio producida en la cascada (Figura $4.17 \mathrm{izq}$ ). Estas emisiones contienen información acerca de todo el desarrollo de la cascada, porque las emisiones de radio se producen en todas las etapas de la lluvia de llegar al suelo.

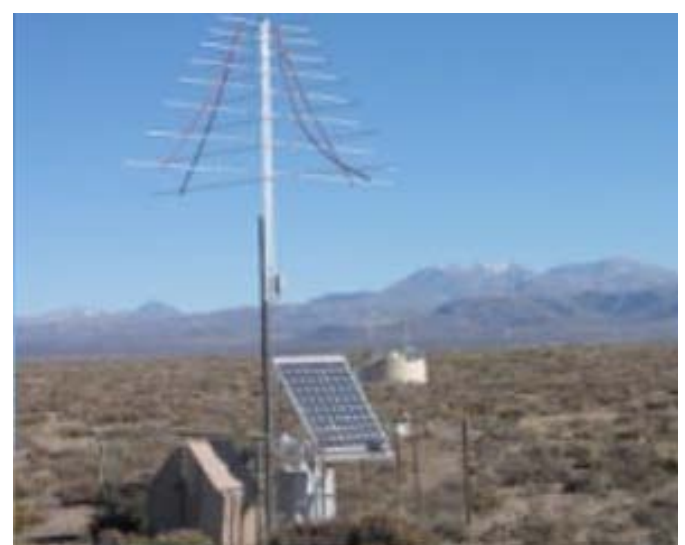

(a) Imagen de una de las antenas de AERA.

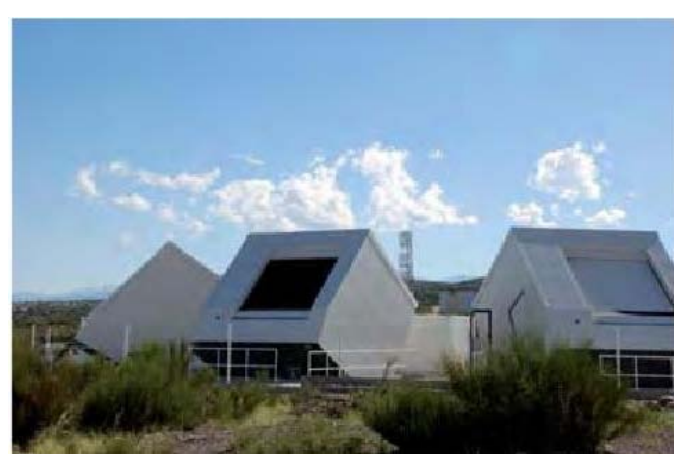

(b) Detalle de los detectores de HEAT.

Figura 4.13: Imágen de una de las antenas de AERA (izq) y de los edificios de HEAT (der). 


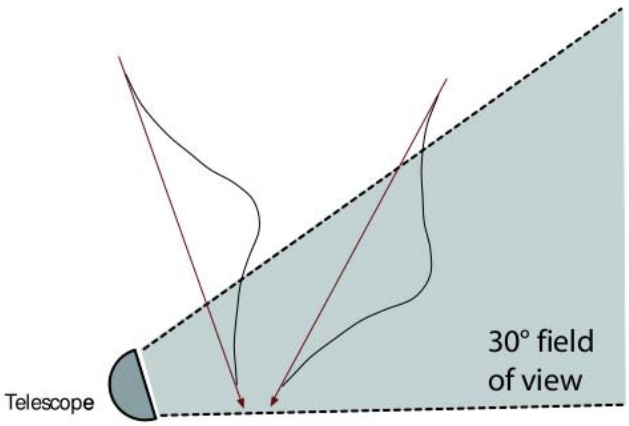

(a) Esquema de la importancia de HEAT para detectar cascadas inclinadas desarrolladas cerca del detector.

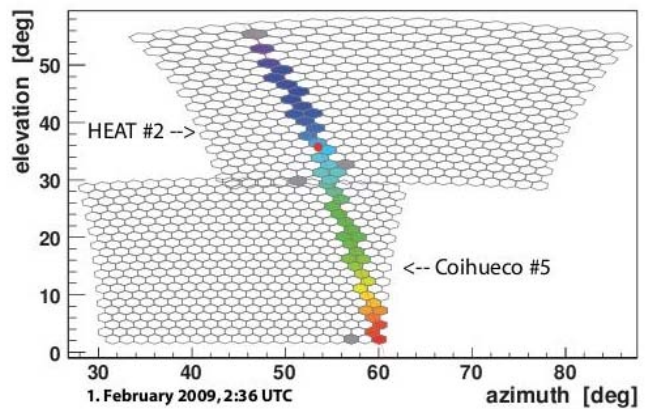

(b) Traza de una cascada desarrollada cerca del detector percibida mediante HEAT y Coihueco.

Figura 4.14: Utilidades de HEAT en la medida de cascadas inclinadas..

El arreglo de detectores RD cubrirá un área de $20 \mathrm{~km}^{2}$ dentro del arreglo SD. Contará con 100 a 150 estaciones con un rendimiento del $100 \%$ de operatibilidad. Particularmente se encuentran en desarrollo los trigger óptimos para correlacionarlos con las medidas SD y FD.

De menor escala, y centrado en medir las primeras instancias de la cascada se encuentra el High Elevation Auger Telescope (HEAT) [86]. Consiste en tres edificios destinados a medir fluorescencia (Figura 4.13 der) localizados cerca de los detectores de FD, extendiendo el campo de visión de los $30^{\circ}$ originales a $60^{\circ}$. Esta gran elevación permite la detección de cascadas de baja energía que se desarrollaron en la alta atmósfera, o lluvias inclinadas desarrolladas por encima del telescopio (Figura 4.14).

Otro de los detectores en desarrollo es el correspondiente al proyecto Auger Muons and Infill for the Ground Array (AMIGA) [87], compuesto por placas de centelleo destinadas a medir la componente muónica de la cascada. Estas placas forman varios niveles, y se encuentran enterradas 2,25 metros con el fin de no detectar las partículas electromagnéticas. Las medidas se complementan con la de dos nuevos subarreglos de detectores Cherenkov, ubicados con este propósito (Figura 4.15). El primero de los subarreglos consta de 42 tanques SD equiespaciados por $750 \mathrm{~m}$ mientras que el segundo consiste en 24 tanques ubicados a 433 m entre sí.

Por último describiremos el detector BATATA, diseñado para caracterizar y medir el flujo de muones, y estudiar la contaminación electromagnética sufrida por los contadores de muones del arreglo AMIGA. Este detector se encuentra cerca de AMIGA, también enterrado, y consiste en tres planos enterrados a diferentes profundidades. Cada uno de esos planos está segmen- 

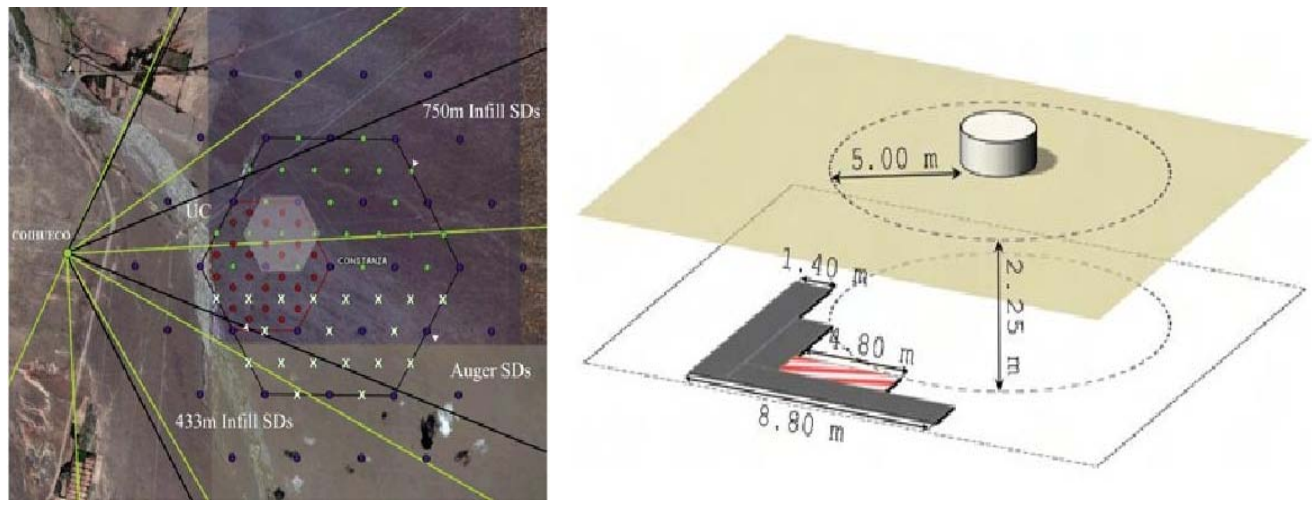

(a) Futura ubicación del arreglo de (b) Esquema del arreglo de AMIGA, enterrado AMIGA, en las cercanías de Coihue-2.25m y en coincidencia con uno de los tanques co. SD. Se muestra una posible configuración para los centelleadores.

Figura 4.15: Detector AMIGA.

tado en un entrecruzamiento de 49 barras conteniendo 49 centelleadores cada una.

\subsection{El monitoreo atmosférico}

Para estimar la energía, mediante el arreglo FD; se necesita una buena caracterización de la atmósfera en el momento de la medida. Recordando lo del capítulo anterior, esto es debido a que la energía se estima a partir del número de fotones que alcanzan la cámara; número que depende de las posibles difracciones por dispersión tipo Rayleigh o Mie. Asimismo la estimación incluye conocer también la energía depositada en el aire, parámetro que depende de la densidad del mismo.

Por otra parte, cuando se utiliza el arreglo de SD, el número de partículas que alcanza el suelo depende de la cantidad materia sobre la que ha tenido que desarrollarse la cascada. Conocer la variación de la densidad con la altura asegura mayor precisión en la determinación de observables. En particular, el número de muones se utiliza para estimar la energía del primario.

En definitiva, los experimentos de rayos cósmicos deben poseer un continuo registro de la atmósfera. Como corresponde, el Observatorio Pierre Auger posee un completo sistema de determinación de variables atmosféricas que se complementan para monitoreo permanente de la atmósfera en el sitio del experimento. Los acrónimos de estos dispositivos son BLS, LIDAR, CLF, XLF, HAM, FRAM; y se encuentran también los dispositivos Monitoreo de 

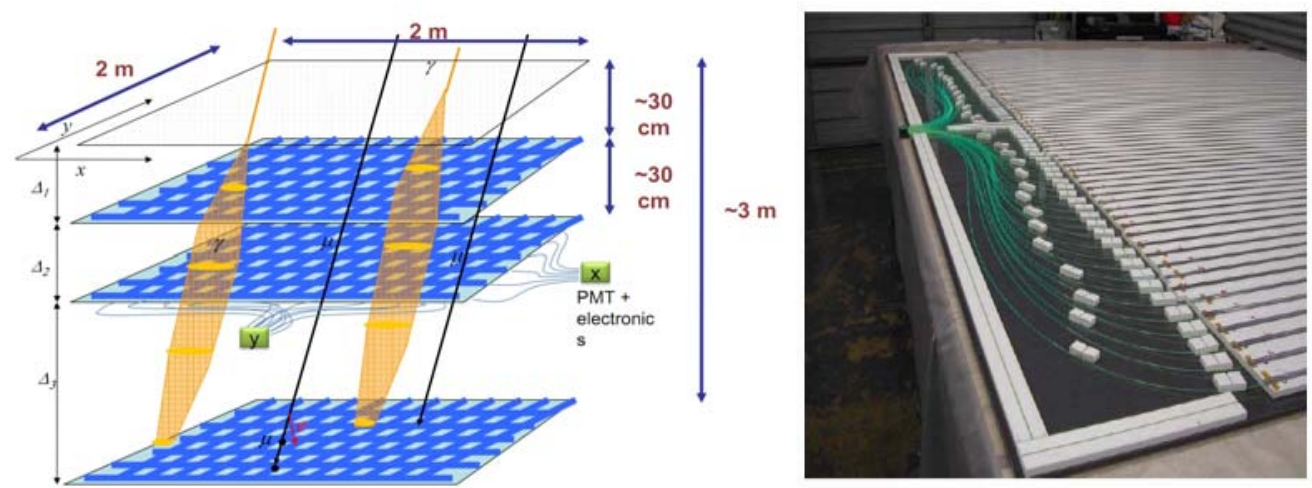

(a) Esquema del detector BATATA, con los tres pla-(b) Uno de los planos del detecnos de observación compuestos por barras entrecru- tor BATATA. Se observan las bazadas con centelleadores. rras con los detectores y las fibras ópticas usadas en el transporte de señal.

Figura 4.16: Detector BATATA.

nubes y las Estaciones meteorológicas.

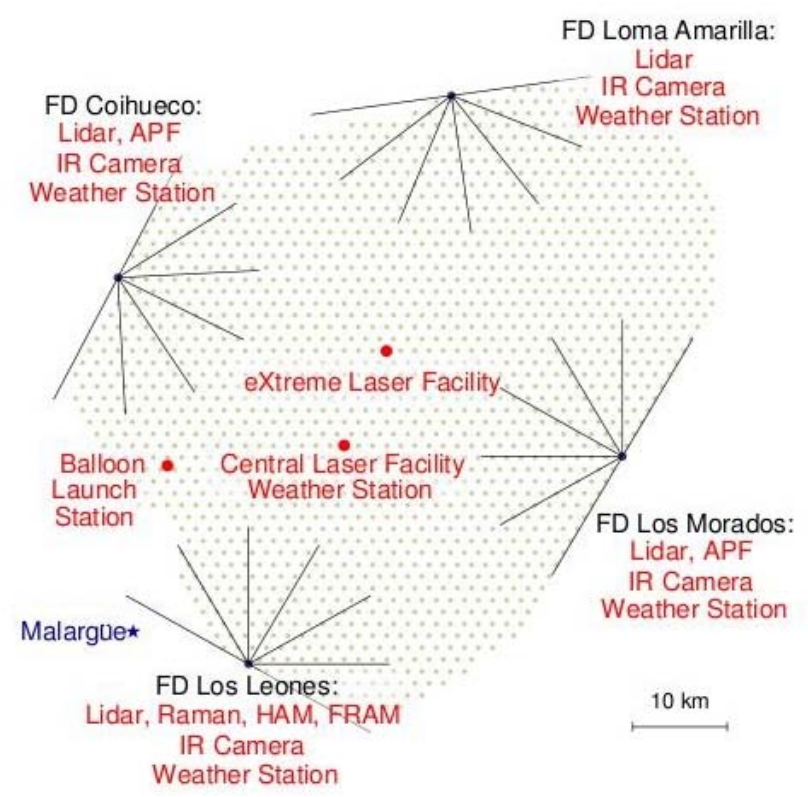

Figura 4.17: Plano de la disposición de los dispositivos que componen el monitoreo atmosférico del Observatorio Pierre Auger. 


\subsubsection{BLS : Estación de lanzamientos de globos}

El nombre BLS proviene de Balloon Launching Station que hace referencia a una estación de lanzamientos de globos atmosféricos que se encuentra en funcionamiento desde Julio de 2005. Consiste en un pequeño refugio (Figura $4.18 \mathrm{izq}$ ) donde se preparan los globos, poniendo a punto in situ los detectores y el gas del globo. Cuenta además con un radio receptor que capta las señales de los globos, una computadora que procesa los datos, una pequeña estación metereológica situada en el techo, y una antena de comunicaciones enlazada con Coihueco.

La estación se encuentra ubicada cercana al borde del arreglo de detectores de superficie entre Coihueco y Los Leones (Figura 4.17). Esta ubicación no es casual, responde al hecho de que el viento predominante proviene del Sud-Oeste y de esta manera las mediciones se realizan por sobre el arreglo de SD.

Los globos que se usan actualmente utilizan una radiosonda Graw DFM97 (Figura 4.18 der) que toma medidas aproximadamente cada 8 s. La radiosonda está adherida a un globo de helio y la altura media que alcanzan los globos es de 20-25 km. Cada punto de medida implica valores de posición, presión, temperatura, humedad relativa y velocidad del viento. La posición y altura se miden gracias a un GPS. La velocidad del viento se calcula a partir de posiciones sucesivas medidas por el GPS. El valor de la presión no se obtiene de medidas directas sino que se deriva del valor medido en el suelo; y la altura determinada por el GPS junto con las medidas de Temperatura y Humedad.

La incerteza en las mediciones de las radiosonda, están referidas en el siguiente cuadro [97]:

\begin{tabular}{|c|c|c|}
\hline Parámetro & Incerteza & Rango \\
\hline \hline Presión & $<1.0 \mathrm{hPa}$ & $5 \mathrm{hPa}$ a $1080 \mathrm{hPa}$ \\
Temperatura & $<0,2{ }^{\circ} \mathrm{C}$ & $-80{ }^{\circ} \mathrm{C}$ a $44{ }^{\circ} \mathrm{C}$ \\
Humedad & $<5 \%$ & $0 \%$ a $105 \%$ \\
Velocidad Viento & $<0,2 \mathrm{~m} / \mathrm{s}$ & Sin Datos \\
\hline
\end{tabular}

Cuadro 4.1: Datos de las incertezas propias de las radiosondas. 


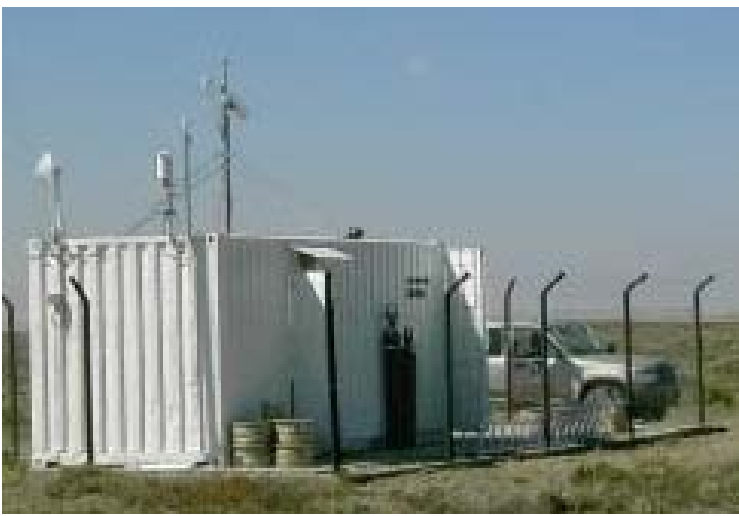

(a) Estación de lanzamiento de globos atmosféricos.

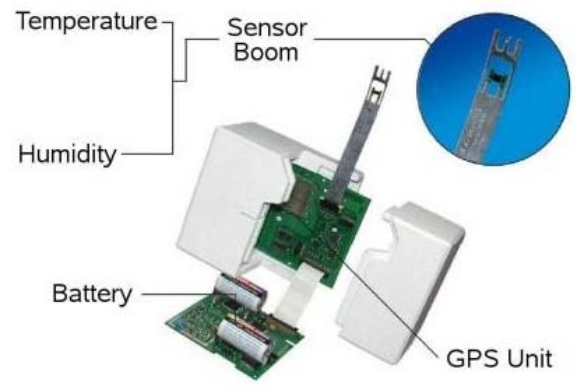

(b) Componentes del modelo de radiosonda actualmente utilizada en los globos atmósfericos

Figura 4.18: Estación de lanzamiento de globos atmosféricos (izq). Nótese la estación metereológica y la antena de comunicaciones en su techo. Equipamiento actual de Radiosonda (der). Como se observa no incluye barómetro, sino que la presión se determina a partir de la altura, humedad y temperatura.

\subsubsection{Las estaciones meteorológicas}

Los parámetros atmosféricos al nivel del suelo están monitoreados permanentemente gracias una serie de estaciones metereológicas situadas junto a cada edifico de los detectores FD y en el centro del arreglo SD. Cada una de ellas ha sido instalada juntamente con el edificio de FD, lo que implicó que 1 apriemra de ellas fuera la de Los Leones en el año 2002. El resto de las estaciones fueron paulatinamente incorporándose al sistema de monitoreo y desde 2007 se encuentran todas las estaciones funcionando.

Las estaciones poseen un equipamiento que cada 5 minutos toma medidas de presión, temperatura, humedad y velocidad del viento. Los rangos y las precisiones de cada uno de los sensores se encuentran en el Cuadro 4.2. 


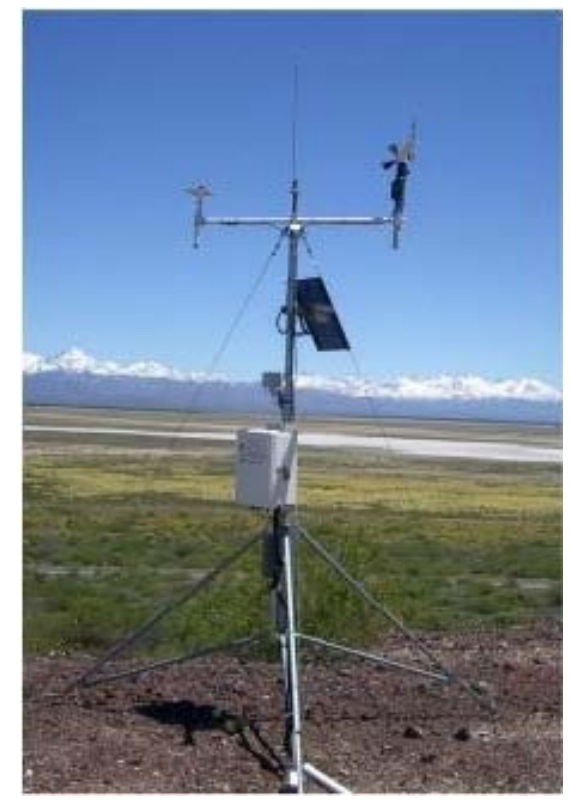

(a) Estación metereológica.

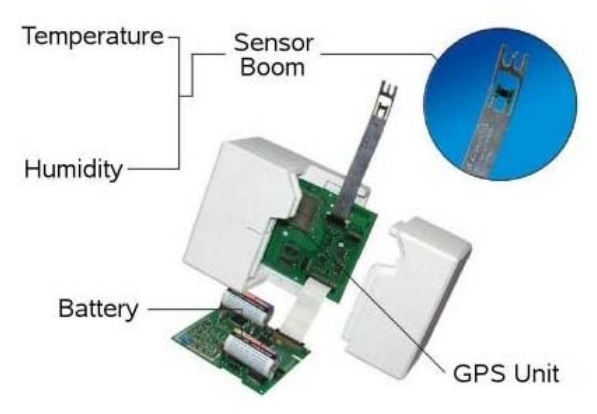

(b) Componentes del modelo de radiosonda actualmente utilizada en los globos atmósfericos

Figura 4.19: Estación metereológica Campbell. Los sensores de temperatura, humedad y velocidad del viento se encuentran en la parte superior junto a la antena. Puede verse el panel solar para el abastecimiento eléctrico y la caja con la electrónica y sensor de presión.

\begin{tabular}{|c|c|c|c|}
\hline Parámetro & Incerteza & Rango & Estabilidad \\
\hline \hline Presión & $1.0 \mathrm{hPa}$ & $600 \mathrm{hPa}-1100 \mathrm{hPa}$ & $0,1 \mathrm{hPa} / \mathrm{año}$ \\
Temperatura & $0,4^{\circ} \mathrm{C}$ & $-40{ }^{\circ} \mathrm{C}-70{ }^{\circ} \mathrm{C}$ & Sin Datos \\
Humedad & $2 \%$ & $0 \%-100 \%$ & $1 \% /$ año \\
Velocidad Viento & $0,1 \mathrm{~m} / \mathrm{s}$ & $0,2 \mathrm{~m} / \mathrm{s}-75 \mathrm{~m} / \mathrm{s}$ & Sin Datos \\
Dirección Viento & $2{ }^{\circ}$ & $0^{\circ}-360^{\circ}$ & Sin Datos \\
\hline
\end{tabular}

Cuadro 4.2: Datos de las incertezas y rangos de mediciones del instrumental de las estaciones metereológicas.

\subsubsection{LIDAR}

Es el acrónimo de LIght Detection and Ranging, y realiza mediciones constantes de la profundidad óptica vertical (VAOD) en cada sitio FD. Esta 
cantidad es importante para determinar la atenuación de luz por dispersión debida a aerosoles presentes en el aire. Este sistema consiste en un láser de $355 \mathrm{~nm}$ que se activa periódicamente por $7 \mathrm{~ns}$ generando un haz en forma vertical [81].

Cuando esta luz incide sobre los aerosoles de la atmósfera, se producen procesos dispersivos que permiten captar nuevamente luz que llega al suelo. Esto se realiza mediante una serie de espejos parabólicos de $40 \mathrm{~cm}$ de radio (Figura 4.20). En el foco de cada uno de los espejos se encuentra un fotomultiplicador que detecta la luz.

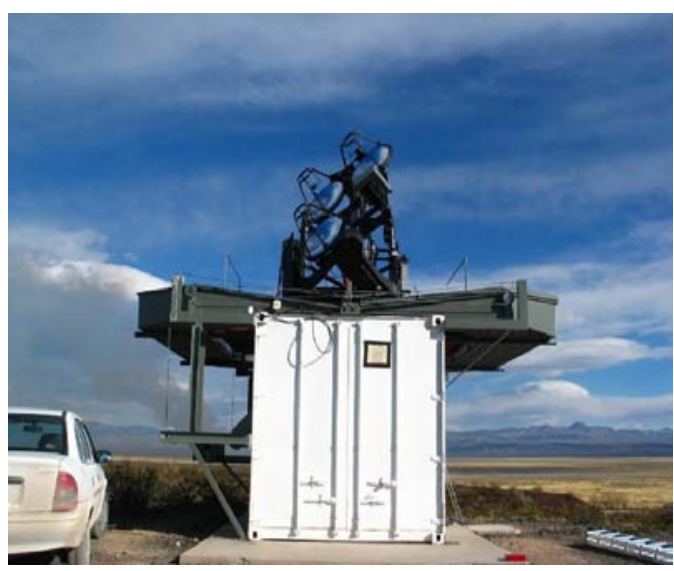

(a) Detector LIDAR abierto.

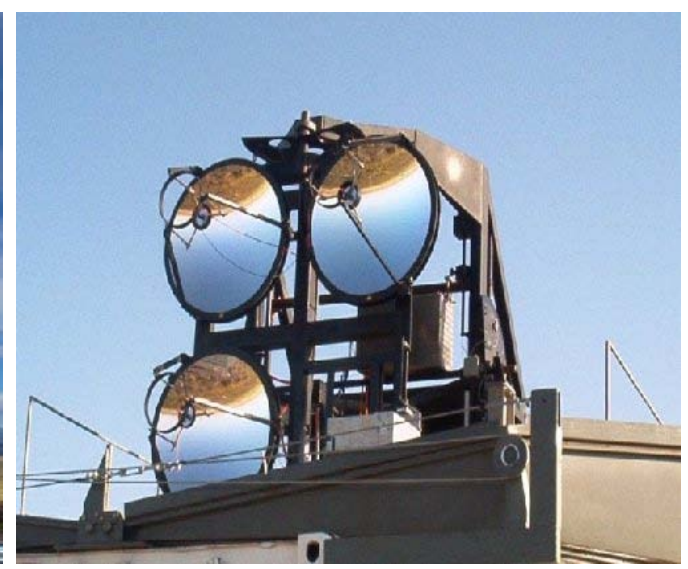

(b) Detalles de los espejos parabólicos.

Figura 4.20: Detector LIDAR.

Cada LIDAR tiene 4 modos de funcionamiento. Tres de ellos son automáticamente ejecutados en las sesiones de medición FD por detrás y delante de la cámara. El cuarto modo corresponde al Shoot-the-Shower (StS), que se dispara cuando un evento híbrido stereo de alta energía es detectado. Está diseñado para medir nubosidad moviéndose en el campo visual del detector FD y consiste en un barrido del haz en el campo de visión [82]. Por lo necesario de hacer el barrido en forma inmediata a la medida, requiere un rápido módulo de reconstrucción $\mathrm{FD}$ que privilegia rapidez a una alta precisión. Es destacable además que cada vez que se dispara este modo LIDAR por un evento ultraenergético, el detector de FD deja de funcionar por 4 minutos para permitir el barrido StS. 


\subsubsection{Central y eXtreme Laser Facilities (CLF/XLF)}

La Instalación Láser Central (CLF) está ubicada en el medio del arreglo de SD. Se encuentra aproximadamente equidistante a tres de los cuatro detectores FD, unos $26 \mathrm{~km}$ de Los Leones y $30 \mathrm{~km}$ de Coihueco y Los Morados [83]. Una segunda instalación, llamada Instalación Láser Extrema (XLF) tiene la misma función que CLF y se ubica equidistantemente de Coihueco, Loma Amarilla y Los Morados.

El instrumento principal es un láser pulsado tipo YAG de $355 \mathrm{~nm}$ y variada intensidad, montado de manera tal que pueda ser dirigido a cualquier sitio por sobre el horizonte. Con este montaje un disparo láser puede ser detectado desde diversos detectores FD por lo que se usa constantemente para calibraciones. Al igual que con el sistema LIDAR, puede ser medida la profundidad óptica de aerosoles, pero la diferencia reside en que no es la luz retrodispersada la utilizada, sino que la luz dispersada en forma lateral y captada en las cámaras FD. Comparando la atenuación sobre diferentes detectores, se determina la uniformidad horizontal de aerosoles.

El láser es disparado en las noches de medida de FD durante 20 veces en forma vertical a $0,5 \mathrm{~Hz}$ cada 15 minutos. Para sincronizar los detectores FD con los SD, un disparo adicional incluye que parte del haz láser es enviado vía fibra óptica al tanque "Celeste" situado en las proximidades. De esta manera se detectan posibles discrepancias temporales.

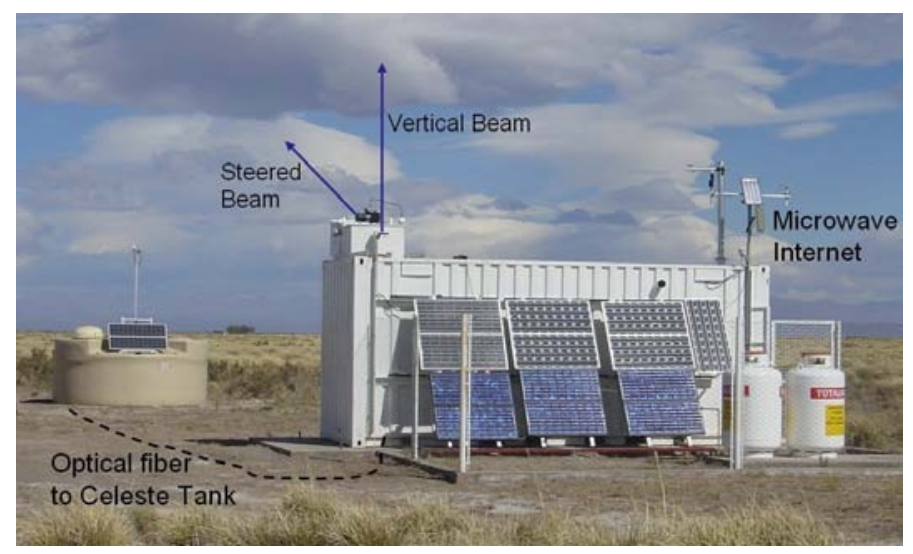

Figura 4.21: Instalación de Láser Central (CLF). Detalle de los componentes del equipamiento.

La energía del disparo láser es reconstruida mediante los detectores FD. Si la energía es reconstruida correctamente, se considerarán confiables el resto de las medidas de lluvias realizadas. Aunque tanto la posición como la dirección 
de la CLF son conocidas, el proceso de reconstrucción puede ser calibrado reconstruyendo la geometría del evento láser.

\subsubsection{Cámaras infrarrojas para nubosidad}

En los sitios de los cuatro detectores FD se encuentra montado un dispositivo con una cámara infrarroja que monitorea la nubosidad por sobre el campo visual del detector El campo visual de cada cámara es de $45^{\circ}$ x $35^{\circ}$ y toma fotografías periódicamente en el rango espectral comprendido entre $700 \mathrm{~nm}$ y $1400 \mathrm{~nm}$ [84]. Durante la adquisición de datos FD, el campo visual correspondiente a todo el detector es analizado cada 5 minutos rotando la cámara IR, de manera tal que todo el cielo se monitorea en 15 minutos.

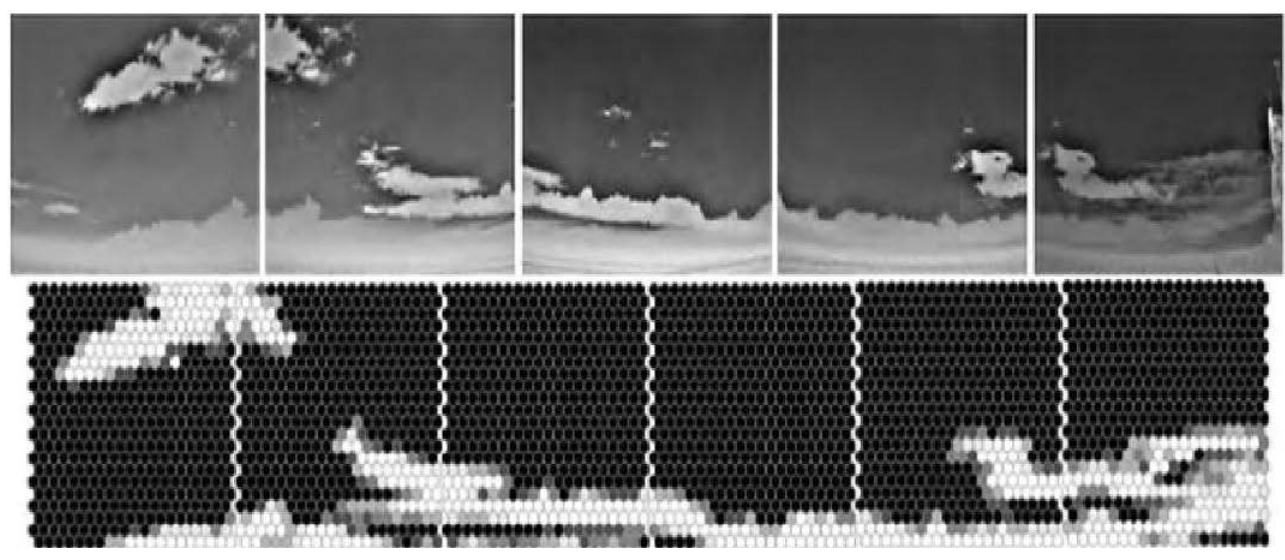

Figura 4.22: En la parte superior se observa la composición de las fotos tomadas por las cámaras infrarrojas para nubosidad. Por debajo se encuentra la proyección sobre los píxeles de cada cámara pudiendo identificar los que se anularán en la reconstrucción.

Usando un software de procesamiento de imágenes, se analizan los datos crudos de la cámara IR para detectar si los píxeles de la cámara FD contiene nubes en su campo visual 4.22. Tras el análisis de esas fotografías puede decidirse la eliminación de la reconstrucción de alguno de los píxeles que han sido identificados. Usando la combinación de fotografías de todos los detectores puede determinarse la cobertura completa de la nubosidad por sobre el arreglo del Observatorio. 


\subsubsection{Otros sistemas de monitoreo atmosférico}

Los anteriores representan los principales dispositivos utlizados para monitorear las condiciones atmosféricas dentro del Observatorio Auger. Sin embargo faltan enumerar una serie de monitores mas pequeños. Ellos son [66]:

- Monitor de fases de aerosol (APF): se utiliza para medir las propiedades de aerosoles en las cercanías de los edificios de FD. Consiste en un haz pulsante colimado de radiación UV que se dispara horizontalmente por frente del campo de visión del detector. Estas medidas se utilizan en determinar la componente dispersada de la radiacion Cherenkov [88]

- Monitor de atenuación horizontal (HAM): mide la atenuación total al nivel de los edificios FD. Consiste en una fuente luminosa ubicada en el edificio FD que emite en dirección a otro de los detectores FD. La radiación es detectada por una cámara CCD con alta exposición y permite medir la atenuación total (molecular y aerosoles) establecida por sobre el arreglo SD. La cámara posee varios filtros para establecer la dependencia con la longitud de onda y toma imágenes una vez por hora en las noches de medición de FD.

- Monitor robótico fotométrico de la atmósfera FRAM: consiste en un telescopio equipado con una cámara CCD y un fotómetro. Con estos dispositivos combinados se monitorea un conjunto de estrellas patrón. Se usa para la correcta calibración, una fuente calibrada ubicada a 50 $\mathrm{km}$ utilizando filtros en varios de longitudes de onda.

\subsection{Adquisición, procesamiento y análisis de datos}

A modo de cierre del proceso de estudio, comentemos brevemente lo que sucede con los datos medidos con los detectores. Con el objeto de optimizar el uso de los datos observados experimentalmente, tanto los detectores como el instrumental de monitoreo se encuentran conectados al centro de adquisición de datos localizado en el edificio central del Observatorio. La comunicación entre los detectores y este sitio se produce a través de señales microondas. Además de concentrarse en primera instancia la información colectada con los arreglos, en CDAS se producen algunos niveles de trigger (ver sección 4.2) )e incluso una primera reconstrucción del evento medido por SD.

La reconstrucción completa del evento se realiza mediante el software oficial de análisis $\overline{O f f l i n e}$ [121], y puede realizarse considerando el modo híbrido 
o sólo los detectores FD o SD; de acuerdo estén los datos disponibles. Básicamente $\overline{O f f l i n e}$ provee un entorno de trabajo para tareas de simulación y reconstrucción, donde los usuarios pueden procesar datos o lluvias simuladas incluyendo además algoritmos de reconstrucción propios o secuencias de instrucciones mediante accesibles interfases al detector y a la información de los eventos. Particularmente estos puntos se facilitan gracias a su estructura modular que permite rápidos cambios.

Se completa la tarea general del Observatorio cuando los resultados de la reconstrucción se envían luego a un Centro de Cálculo (Centre de Calcul de l'Institut National de Physique Nucleaire et de Physique des Particles) ubicado en Lyon (Francia), donde se encuentran disponibles para el análisis pormenorizado de cada una de las líneas de investigación del Observatorio. 


\section{Capítulo 5}

\section{Los perfiles de profundidad atmosférica}

En el análisis de lluvias generadas por rayos cósmicos es muy importante tener una precisa caracterización de la atmósfera. Esto es debido a que, como se planteó anteriormente, la lluvia de partículas se produce por la interacción del primario y los secundarios con los núcleos de los átomos del aire, lo que conlleva a diferentes probabilidades de interacción de acuerdo a las diferentes densidades.

El parámetro natural utilizado para representar la cantidad de materia atravesada por una partícula que ingresa a la atmósfera es la profundidad atmosférica, identificada con la letra $X$ y definida como

$$
X(h)=\int_{h}^{\infty} \rho(\zeta) d \zeta
$$

donde $h$ representa la altura medida desde el nivel del mar y $\rho(\zeta)$ es la densidad del aire a la altura $\zeta$, también medida desde el suelo. A partir de la expresión (5.1) es evidente que el valor máximo de $X$ se corresponde con el nivel del suelo y el nulo con los comienzos exteriores de la atmósfera.

\subsection{Breve descripción de la Atmósfera.}

Antes de continuar en la interiorización de los perfiles de profundidad atmosférica, es conveniente una pequeña descripción de la atmósfera que contextualice lo que en definitiva es el objeto de estudio.

Se denomina atmósfera a la masa inhomogénea gaseosa que rodea a la Tierra. Es un sistema con una dinámica constante y de muy difícil predicción. Esto es debido a que la mayoría de los procesos físicos en la atmósfera están 
ligados a la radiación solar y su correlación con la rotación de la Tierra tanto alrededor de su propio eje como alrededor del Sol. Estos dos movimientos de rotación de la Tierra, sumado a la dinámica de los fluidos, conducen a cambios en la influencia de la radiación solar que se reconocen en variaciones estacionales, diarias y zonales [90].

Los modelos que representen a la atmósfera, entre ellos los que describen alguna de sus características como la profundidad atmosférica, deben contemplar inexorablemente la inhomogeneidad del aire. De hecho, la distribución de aire es tan extrema que aproximadamente el $90 \%$ de la masa se encuentra en los primeros $18 \mathrm{~km}$ por encima del nivel del mar y sólo el $1 \%$ por encima de los $32 \mathrm{~km}$. Este hecho repercute evidentemente en la densidad del aire, que disminuye seis órdenes de magnitud desde el nivel del mar hasta los primeros $100 \mathrm{~km}$ de altitud, y otros seis órdenes de magnitud entre los $100 \mathrm{~km}$ y $300 \mathrm{~km}$ de altitud.

La composición química es otro de los parámetros relevantes a tener en cuenta, y se expresa usualmente mediante la masa molar $M_{m}$. El valor promedio en el aire seco es aproximadamente de $M_{m}=28.966 \mathrm{~g} \mathrm{~mol}^{-1}$, y representa la mezcla atómica de aproximadamente $78.08 \%$ de Nitrógeno, $20.94 \%$ de Oxígeno, $0.93 \%$ de Argón y $0.05 \%$ de otros elementos (Cuadro 5.1). La masa molar tiene también una particular variación vertical: permanece prácticamente constante en el rango de alturas $h^{1}$ comprendido entre $0 \mathrm{~km} \leq h \leq$ $90 \mathrm{~km}$, y comienza a decrecer monótonamente para alturas mayores (Figura 5.1). Debido a esta sustancial diferencia en la composición del aire se establecen dos capas de atmósfera, la comprendida entre $0 \mathrm{~km} \leq h \leq 90 \mathrm{~km}$ que se denomina homósfera, y la situada por encima de los $90 \mathrm{~km}$ que se denomina heterósfera.

Dentro de las variables de estado, la temperatura es probablemente la que tiene un comportamiento más ecléctico. Su variación es debida a diversos factores como el flujo de calor por la posición del sol o presencia de nubes, procesos de intercambio de calor entre la superficie terrestre y la atmósfera, desplazamiento de masas por viento, topografía, tipo de superficie terrestre (hielo, agua, desierto, etc.)[89]. La variación vertical de la temperatura (Figura 5.2) suele ser modelizada en estratos como se relata a continuación [91]:

- Disminuye linealmente con la altitud en la zona denominada tropósfera, con una razón comprendida entre $5.5^{\circ} \mathrm{C} / \mathrm{km}$ y $6.5^{\circ} \mathrm{C} / \mathrm{km}$ en los primeros 9 - $12 \mathrm{~km}$.

- Se mantiene aproximadamente constante entre los 9 y $15 \mathrm{~km}$. Esta capa

\footnotetext{
${ }^{1}$ Consideraremos a la altura siempre medida en $\mathrm{km}$, con el valor 0 al nivel del mar
} 


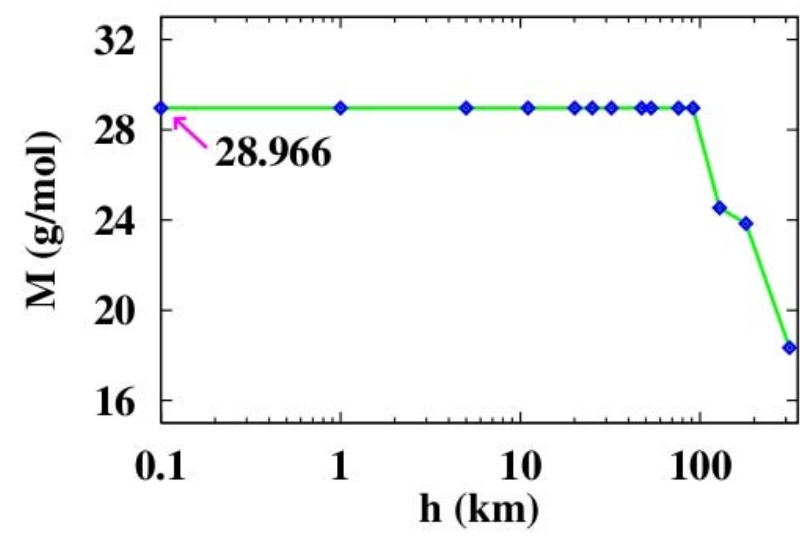

Figura 5.1: Masa molar media del aire seco en la atmosféra expresado en función de la altura [100]. Los puntos corresponden a medidas de la USStdA [99]. La línea solo es una ayuda visual.

se denomina tropopausa.

- Aumenta linealmente con la altura hasta los 45 - $50 \mathrm{~km}$, en la denominada estratósfera, llegando hasta casi los $0{ }^{\circ} \mathrm{C}$.

- No cambia durante unos pocos kilómetros, aproximadamente entre los 45 - $55 \mathrm{~km}$, en la denominada capa de interfase estratopausa.

- Disminuye linealmente hasta los $90 \mathrm{~km}$ alcanzando valores cercanos a los $-90{ }^{\circ} \mathrm{C}$. Este estrato se denomina mesósfera.

- Tras mantenerse prácticamente constante en otra capa de interfase llamada termopausa, aumenta linealmente con la temperatura hasta por valores encima de $0{ }^{\circ} \mathrm{C}$ cuando se encuentra a $110 \mathrm{~km}$ en la llamada termósfera.

- Luego, dentro de la termósfera, debido a que no hay filtración de la radiación solar ultravioleta, gamma y X, se produce una alta ionización de átomos y molculas, alcanzándose valores cercanos a los $1000{ }^{\circ} \mathrm{C}$.

- Debido a los bajísimos valores de densidad, por encima de esa altitud la temperatura no está definida de acuerdo al concepto termodinámicoestadístico de la misma. Tanto el resto de la termósfera (que se extiende hasta aproximadamente $700 \mathrm{~km}$, como la última capa de la atmósfera denominada exósfera (que se extiende hasta los $10000 \mathrm{~km}$ ), se consideran sin temperatura definida. 


\begin{tabular}{|c|c|c|}
\hline Elemento & $\begin{array}{c}\text { Partes por Millón } \\
{[\mathbf{p p m v}]}\end{array}$ & $\begin{array}{c}\text { Masa molar } \\
{[\mathbf{g} / \mathbf{m o l}]}\end{array}$ \\
\hline \hline Nitrógeno $\left(\mathrm{N}_{2}\right)$ & $780840(78.0840 \%)$ & 28.014 \\
Oxígeno $\left(\mathrm{O}_{2}\right)$ & $209460(20.9460 \%)$ & 32.998 \\
Argón $(\mathrm{Ar})$ & $9340(0.9340 \%)$ & 39.948 \\
Neón $(\mathrm{Ne})$ & $385(0.0385 \%)$ & 44.010 \\
Helio $(\mathrm{He})$ & 18.18 & 20.180 \\
Metano $(\mathrm{CH})$ & 5.24 & 4.0026 \\
Kriptón $\left(\mathrm{Kr}_{4}\right)$ & 1.745 & 16.042 \\
Hidrógeno $\left(\mathrm{H}_{2}\right)$ & 1.14 & 83.798 \\
Óxido Nitroso $\left(\mathrm{N}_{2} \mathrm{O}\right)$ & 0.55 & 4.0158 \\
Xenón $\left(\mathrm{Xe}^{2}\right)$ & 0.3 & 131.29 \\
Ozono $\left(\mathrm{O}_{3}\right)$ & 0.09 & 47.998 \\
Iodo $(\mathrm{I})$ & $0.0-0.07$ & 46.006 \\
Dióxido de Nitrógeno $(\mathrm{NO})$ & 0.02 & 126.90 \\
Monóxido de Carbono $(\mathrm{CO})$ & 0.01 & 17.031 \\
Amoníaco $\left(\mathrm{NH}_{3}\right)$ & traza & 18.015 \\
Vapor de agua $\left(\mathrm{H}_{2} \mathrm{O}\right)$ & traza & \\
\hline
\end{tabular}

Cuadro 5.1: Valores medios de los constituyentes de la baja atmósfera [93].

La temperatura, la presión y la composición molecular, son la base para construir las funciones de estado que caracterizan a la atmósfera. En el capítulo siguiente se desarrollará cómo, con estas y otras variables, se construyen los perfiles de densidad, y consecuentemente los de profundidad atmosférica.

\subsection{Los Modelos Clásicos}

En esta sección daremos un breve recorrido sobre los diferentes modelos que consideran a la profundidad atmosférica. Están ordenados de acuerdo a su desarrollo cronológico, aunque como suele ocurrir con la construcción de modelos físicos, también es un orden creciente en complejidad.

\subsubsection{Modelo Isotérmico}

Teniendo en cuenta las características propias de la atmósfera terrestre, históricamente se han ido desarrollando varios modelos para describirla apropiadamente. El modelo más simple que describe la variación de la densidad con la altitud es conocido como modelo isotérmico. Este modelo parte de considerar al aire como un gas ideal, reescribiendo la ecuación de estado 


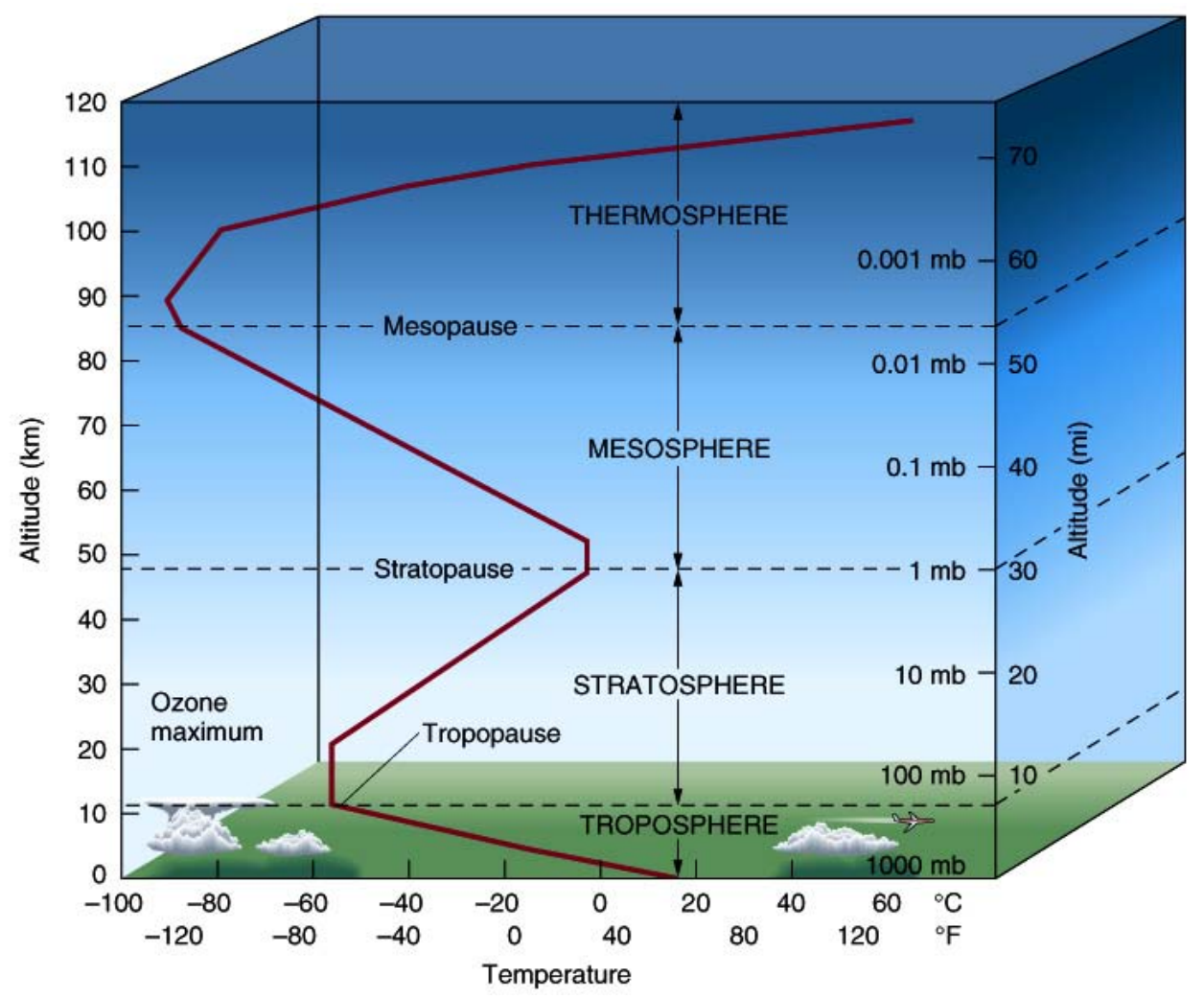

Figura 5.2: Variación vertical estimada de la temperatura en la atmósfera [92]. 
como

$$
\rho=\frac{M_{m} p}{R T}
$$

donde $M_{m}$ representa la ya establecida masa molar, $p$ la presión atmosférica, $T$ la temperatura y $R$ la constante universal de los gases. Considerando la variación de la presión con la altura descrita mediante el Teorema General de la Fluidostática, podemos reescribir este último como:

$$
\frac{d p}{d h}=-\rho g
$$

donde $h$ es la altura medida desde el suelo y $g$ la aceleración local de la gravedad. Juntando estas dos últimas expresiones, podemos resolver la ecuación diferencial

$$
\frac{d}{d h}\left(\frac{\rho R T}{M_{m}}\right)=-\rho g
$$

Suponiendo $M_{m}$ y $T$ constantes, propio de lo isotérmico del modelo, arribamos a la expresión

$$
\rho(h)=\rho_{0} e^{\frac{-g M_{m}}{R T} h}
$$

donde $\rho_{0}$ representa la densidad del aire al nivel del mar y $T$ se toma usualmente también al nivel del mar.

Utilizando esta última expresión (5.5) en la definición (5.1) obtenemos el perfil de profundidad atmosférico propuesto desde el modelo isotérmico, que está dado por

$$
X(h)=X_{0} e^{\frac{-g M_{m}}{R T} h}
$$

donde $X_{0}$ es es el valor de la profundidad atmosférica al nivel del mar y está dado por $X_{0}=\rho_{0} R T / M_{m} g$.

En la búsqueda de contrastar las predicciones del modelo con datos experimentales, se suele recurrir a la base de datos más completa desde comienzos de los años 50 denominada la United States Standard Atmosphere (USStdA). Esta base de datos está confeccionada en base a una gran cantidad de registros realizada en diversos puntos de latitud media del mundo, la mayoría de ellos en los Estados Unidos. Con las medidas en diferentes períodos de tiempo el equipo de estudios atmosféricos de la NASA ha formulado una atmósfera standard. Un primer chequeo del modelo isotérmico (con $\rho_{0}=1,225 \mathrm{~kg} \mathrm{~m}^{-3}$ y $T=288 \mathrm{~K}$ ) muestra que la diferencia entre sus predicciones y los datos experimentales de la USStdA de 1976, es mínima en los primeros kilómetros pero comienza a hacerse notable para altitudes mayores a $10 \mathrm{~km}$. 


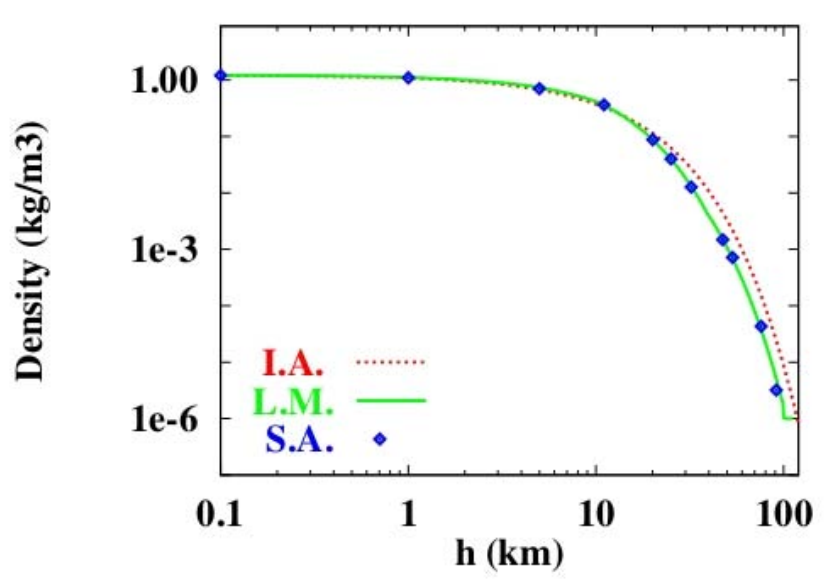

Figura 5.3: Perfil de la densidad del aire en la atmósfera. Los puntos azules representan los datos de la USStdA [99], la línea roja la parametrización correspondiente al modelo isotérmico (tomando $\rho_{0}=1,225 \mathrm{~kg} \mathrm{~m}^{-3}$ y $\mathrm{T}=288$ $K)$, y la línea verde representa la parametrización del modelo de Linsley de 5 capas (obtenido de [100]).

\subsubsection{Modelo de Linsley}

En los comienzos de la década de 1980, John Linsley desarrolló un nuevo modelo sobre la base del modelo isotérmico [94]. Manteniendo la idea de modeli zar al aire como un gas ideal y tratándolo dentro de la teoría clásica de fluidos, delineó un modelo cuya principal característica lo constituye la idea de suponer a la totalidad de la atmósfera como una suma de $L$ capas fijas. En realidad, el estratificar la atmósfera es una idea ampliamente compartida; pero la novedad de la propuesta de Linsley reside en parametrizar independientemente a cada una de las capas. Específicamente se trata de modelizar a las capas como isotérmicas de acuerdo a una temperatura característica y por esa razón se lo reconoce al modelo de Linsley como Modelo isotérmico por capas.

Dentro de este modelo, cada una de las capas isotérmicas se expresa en términos de una función de la forma

$$
X(h)=a+b e^{-\frac{h}{c}}
$$

donde $a, b$ y $c$ son parámetros fijos. Con esta idea, el perfil vertical de pro- 
fundidad atmosférica modelizado por $L-1$ capas isotérmicas está dado por

$$
X(h)=\left\{\begin{array}{ccc}
a_{l}+b_{l} e^{-\frac{h}{c_{l}}} & h_{l-1} \leq h<h_{l} \\
a_{L}-b_{L} \frac{h}{c_{L}} & h_{L-1} \leq h<h_{L} \\
0 & h \geq h_{L}
\end{array} \quad(l=1, \ldots, L-1)\right.
$$

donde $a_{l}, b_{l}, c_{l}$ son parámetros dimensionales que definen la función en la capa $l$ con altitudes $h_{l-1}$ y $h_{l}$. De la expresión de $X(h)$ puede deducirse una muy importante condición de contorno: en la capa situada a mayor altitud, identificada con el subíndice $L$, el modelo fija el valor de $X$ a $0 \mathrm{~g} \mathrm{~cm}^{-2}$ a la altitud de $h_{\max }=h_{L}$. El comportamiento lineal de la función $X(h)$ en esta última capa, diferente a las capas anteriores, no posee ningún significado físico particular y se plantea de esta manera para reducir al valor nulo el valor de la profundidad atmosférica manteniendo continuidad en la función $X(h)$. Si bien los lmites no son precisos, la atmsfera se extiende mucho ms all que el lmite de 112,8 km fijado en forma arbitraria en el modelo de Linsley, y la razn por la cual dicho modelo fija ese lmite debe buscarse en que el mismo fue pensado en el mbito del estudio de rayos cósmicos. En efecto, a los 112.8 $\mathrm{km}$ el valor de $X$ es tan pequeño que fijarlo a 0 por encima de este límite es coherente cuando se analizan cascadas de partículas producidas por rayos cósmicos.

Uno de los puntos de mucho interés que posee la expresión del modelo de Linsley es la reversibilidad. Efectivamente, la función (5.8) puede invertirse permitiendo expresar analíticamente la altura $h$ como función de la profundidad atmosférica $X$ de la manera

$$
h(X)=\left\{\begin{array}{cc}
-c_{l} \ln \left(\frac{X-a_{l}}{b_{l}}\right) & X_{l+1}<X \leq X_{l} \quad(l=1, \ldots, L-1) \\
c_{L}\left(\frac{a_{L}-X}{b_{L}}\right) & 0<X \leq X_{L}
\end{array}\right.
$$

Esta propiedad, que tal vez parezca menor, es muy útil en el manejo de datos, simulaciones y reconstrucciones; y la hacen muy adecuada en el diseño de software específico.

Con el modelo propuesto en (5.8), y la elección de 5 capas y sus límites, Linsley parametrizó los valores de la USStdA obteniendo una serie de valores de referencia de los parámetros $a_{l}, b_{l}$ y $c_{l}$ (Cuadro 5.2). 


\begin{tabular}{|c|cc|ccc|}
\hline $\begin{array}{c}\text { Capa } \\
l\end{array}$ & $\begin{array}{c}\text { Límites } \\
h_{l-1}[\mathrm{~km}]\end{array}$ & $\begin{array}{c}\text { de capa } \\
h_{l}[\mathrm{~km}]\end{array}$ & $\begin{array}{c}a_{l} \\
{\left[\mathrm{~g} \mathrm{~cm}^{-2}\right]}\end{array}$ & $\begin{array}{c}b_{l} \\
{\left[\mathrm{~g} \mathrm{~cm}^{-2}\right]}\end{array}$ & $\begin{array}{c}c_{l} \\
{[\mathrm{~m}]}\end{array}$ \\
\hline 1 & 0 & 4 & -186.5562 & 1222.6562 & 9941.8638 \\
2 & 4 & 10 & -94.9199 & 1144.9069 & 8781.5355 \\
3 & 10 & 40 & 0.61289 & 1305.5948 & 6361.4304 \\
4 & 40 & 100 & 0.0 & 540.1778 & 7721.7016 \\
5 & 100 & 112.8 & 0.01128292 & 1.0 & 10000000 \\
\hline
\end{tabular}

Cuadro 5.2: Valores de la parametrización de Linsley de 5 capas.

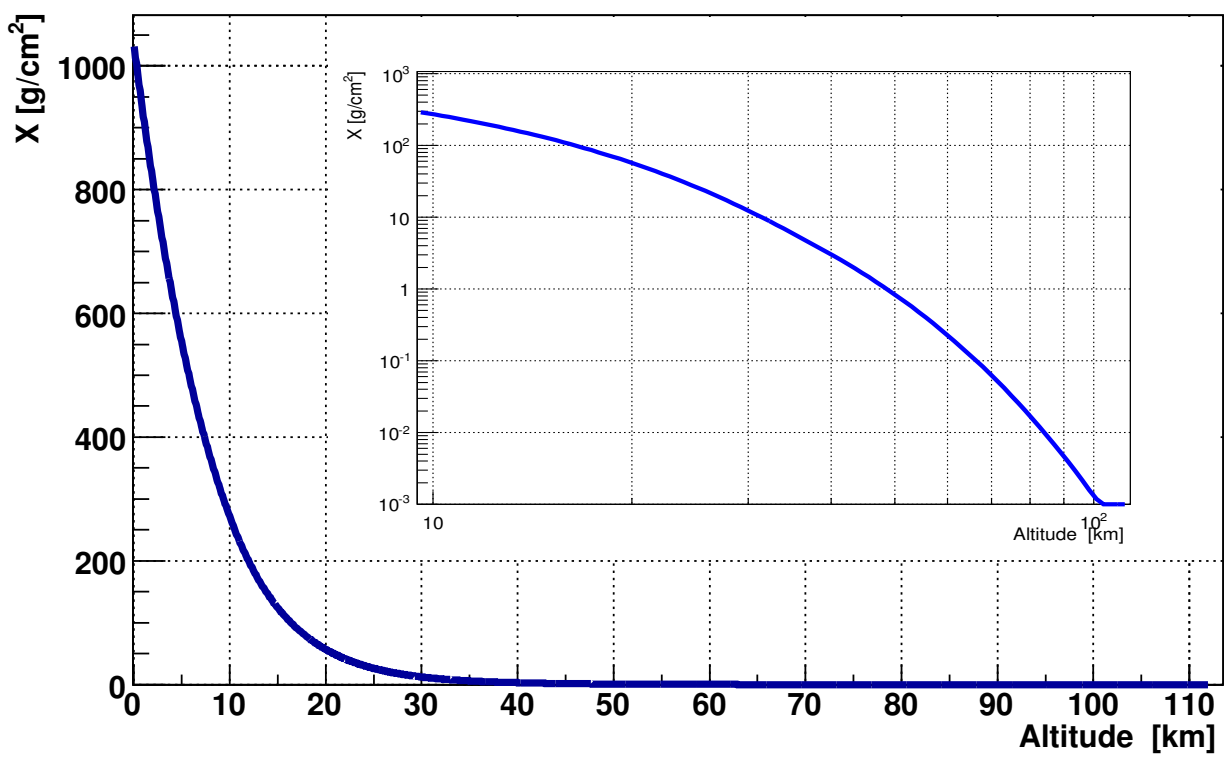

Figura 5.4: Perfil de profundidad atmosférico del Modelo de Linsley de 5 capas en escala lineal y logarítmica.

Con estos valores, el modelo de Linsley cubre prácticamente por completo la atmósfera, ya que el valor $h_{\max }=112.8 \mathrm{~km}$ indicado como límite superior se corresponde con un valor de $X$ menor a $0.001 \mathrm{~g} \mathrm{~cm}^{-2}$ (Figura 5.4).

Los valores determinados por Linsley (Cuadro 5.2) conjuntamente con la expresión (5.8) con $L=5$ ajustan con gran precisión los valores experimentales de la USStdA (1976). Por tal motivo, esta parametrización y la propuesta de caracterización de la atmosféra mediante 5 capas isotérmicas, recibe el nombre de Modelo de Linsley de 5 capas, habiéndose constituido en un modelo ampliamente utilizado. 


\subsection{Modelos recientes}

\subsection{1. $\quad$ El Modelo Mensual (Malargüe Monthly Model)}

Es evidente que los modelos anteriormente descriptos no tienen en cuenta los cambios en Temperatura, presión y humedad que implican cambios en la densidad y consecuentemente alteran el valor de la profundidad atmosférica. Estos cambios, producidos en forma diferente de acuerdo al día y la ubicación geográfica, han sido contemplados en modelos actuales.

En particular, miembros del equipo de monitoreo atmosférico del Observatorio Pierre Auger cuantificaron importantes variaciones estacionales en los perfiles de profundidad atmosférica en la zona de Malargüe [97].

A partir de la base de datos de UK Metereological Office [98], que contiene datos de globos con radiosondas de muchos lugares del planeta, establecieron un perfil promedio de invierno y otro de verano en base a medidas de varios años. Los lugares estudiados, escogidos por su disponibilidad de datos, fueron Mendoza (Argentina) y Salt Lake City (USA). ${ }^{2}$

Entre otros resultados identificaron una diferencia máxima entre el perfil de profundidad atmósferica estacionales y el de Linsley del orden de $20 \mathrm{~g}$ $\mathrm{cm}^{-2}$ (Figuras 5.5 y 5.6 ).
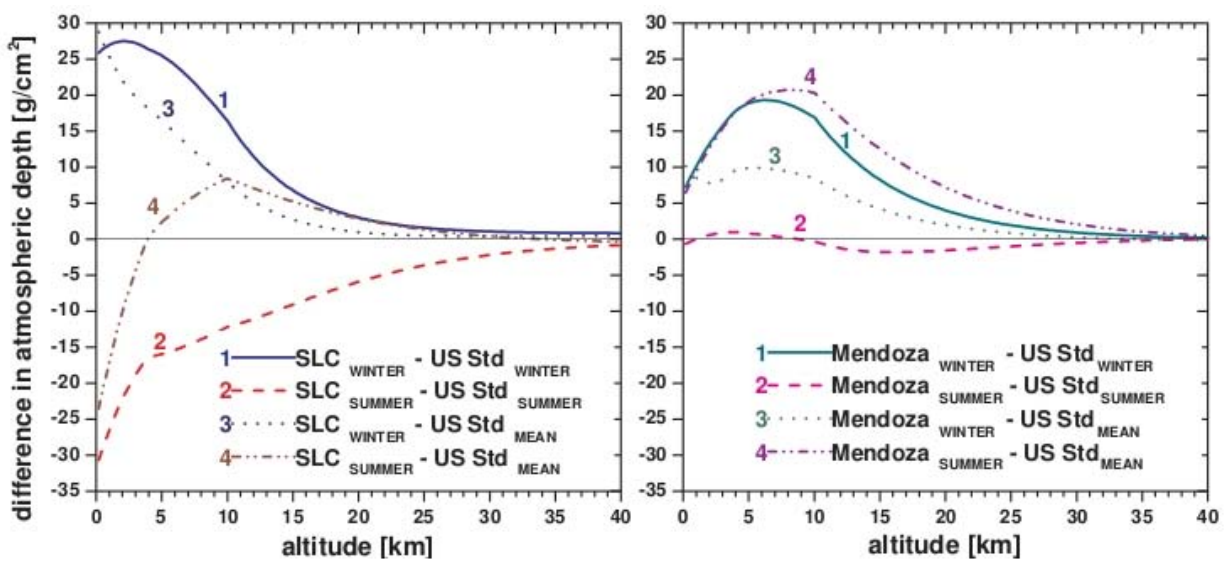

Figura 5.5: Diferencias en la profundidad atmosférica entre la USStda y las medidas promedio estacionales de Salt Lake City (SLC) y Mendoza para los casos invierno y verano.

\footnotetext{
${ }^{2}$ La elección de este último lugar estaba ligado a que en la época del estudio se pensaba [97] construir el sitio Norte del Observatorio Pierre Auger en el estado de Utah (USA).
} 

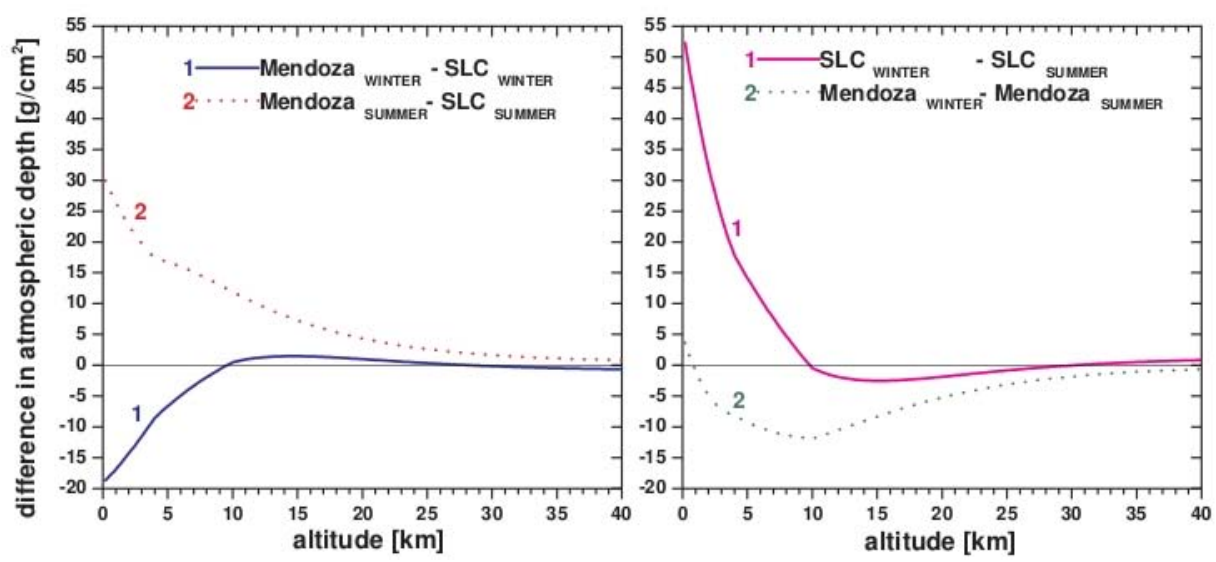

Figura 5.6: Diferencias en la profundidad atmosférica promedio entre las estaciones invierno y verano en los sitios de Salt Lake City (SLC) y Mendoza.

En esa investigación [97] se concluye que un modelo atmosférico global no resulta satisfactorio para el estudio de rayos cósmicos a partir de cascadas; y sugieren fuertemente que a partir de medidas locales se construyan perfiles representativos que consideren las variaciones estacionales y, de ser posible, también las diarias.

Estas conclusiones y resultados motivaron el desarrollo de un nuevo modelo pensado para el sitio del Observatorio Auger. Este modelo establecía 12 parametrizaciones para correspondientes perfiles de profundidad atmosférica, uno por cada mes calendario, y por tal motivo fue nombrado Malargüe Monthly Model (MMM).

Los datos utilizados para el desarrollo del modelo correspondieron a mediciones periódicas de globos atmosféricos lanzados por centros metereológicos de las ciudades de Santa Rosa (La Pampa) y Córdoba; así como a medidas de globos $^{3}$ lanzados en Malargüe por miembros del Observatorio Pierre Auger en el período Agosto 2002 a Noviembre 2004.

El modelo también se estructura detrás de la función de Linsley (5.8) pero ajustando los datos medidos en el Observatorio Auger. Cabe destacar también que considera a la atmósfera como compuesta por 5 capas y en el ajuste, los límites de las capas son libres. Las desviaciones estándar en cada uno de los parámetros son las que permiten cubrir las variaciones día a día.

En las figuras 5.7, 5.8 y 5.9 pueden observarse perfiles de Temperatura, densidad y profundidad atmosférica propuestos por el modelo [95].

\footnotetext{
${ }^{3} \mathrm{La}$ descripción acerca de la adquisición de datos mediante globos atmosféricos y el tratamiento de esas medidas se encuentra en el siguiente capítulo.
} 


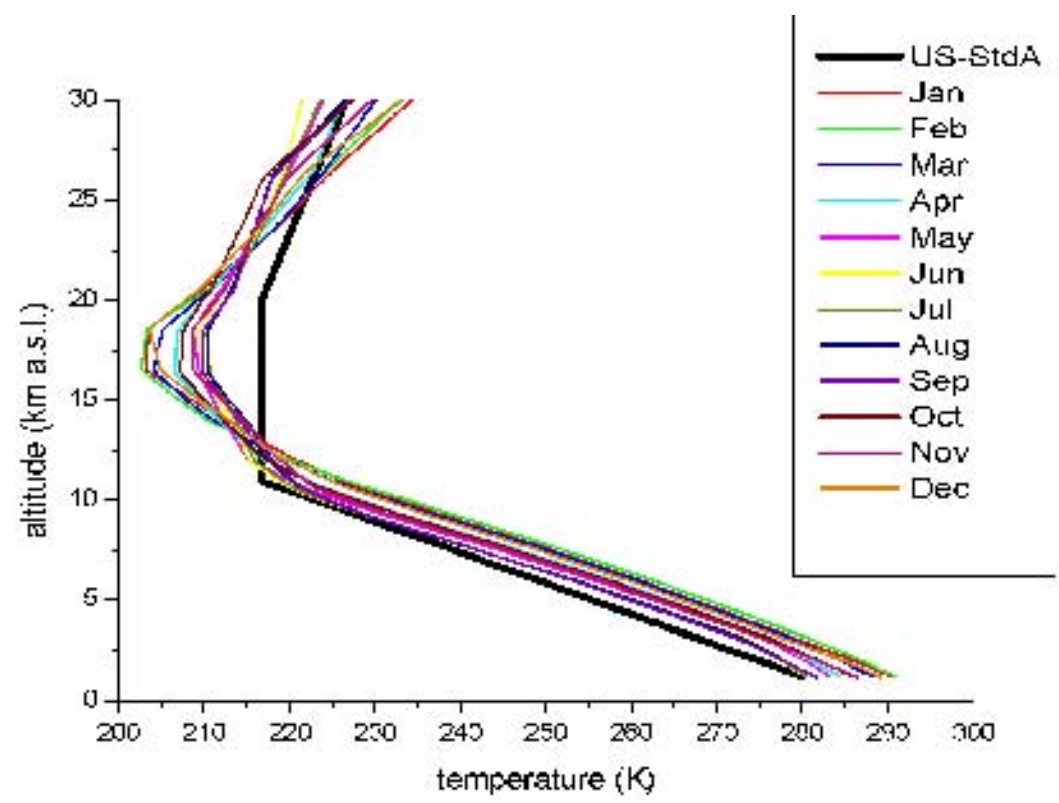

Figura 5.7: Perfiles de Temperatura del Malargüe Monthly Model.

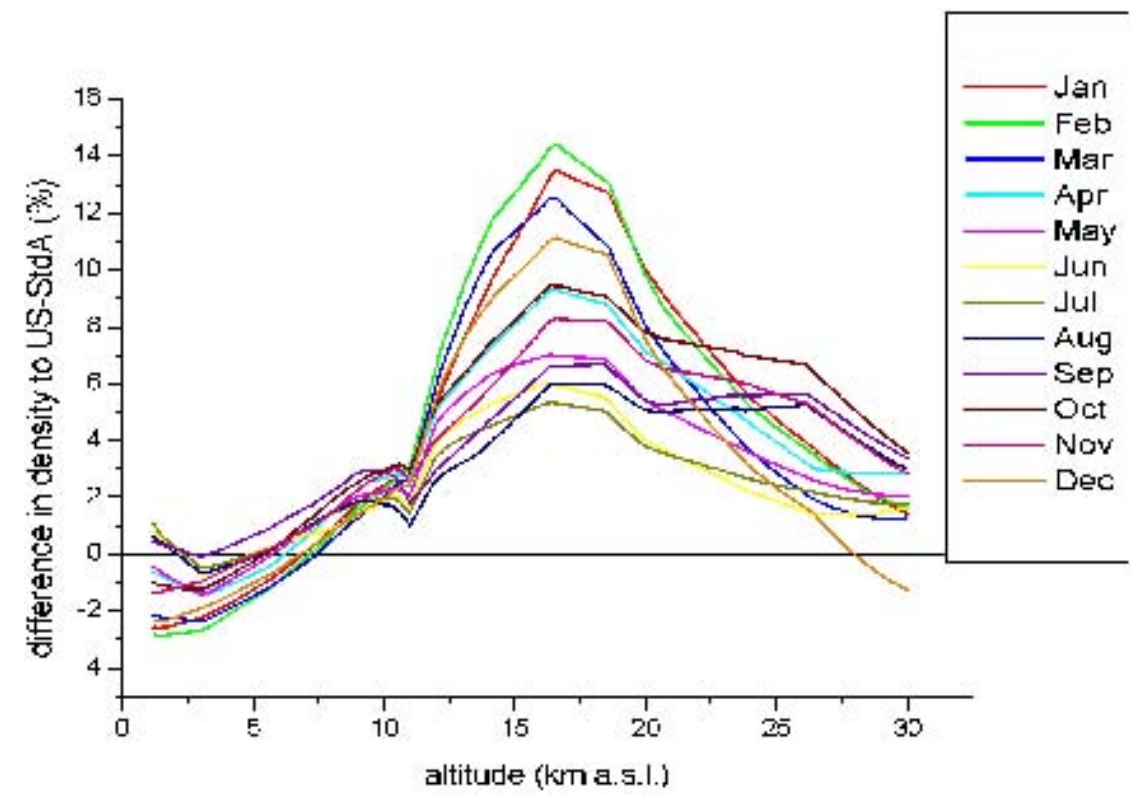

Figura 5.8: Diferencia entre los perfiles de densidad mensual promedio del Malargüe Monthly Model y los datos de la USStdA. 


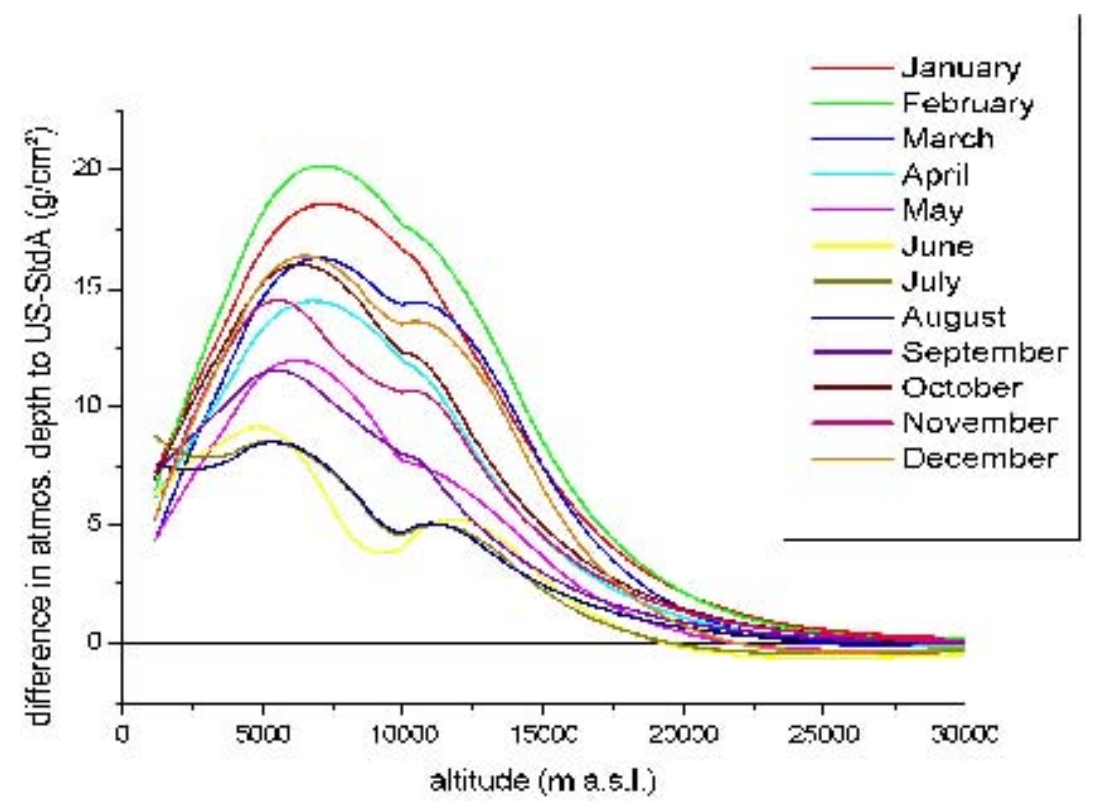

Figura 5.9: Diferencia entre los perfiles de profundidad atmosférica del Malargüe Monthly Model y su comparación con los datos de la USStdA.

Nos detendremos brevemente en el análisis de los perfiles de profundidad atmosférica resultantes (Figura 5.9), donde surge una evidente diferencia con la parametrización de la USStdA hasta $20 \mathrm{~g} \mathrm{~cm}^{-2}$. Como se observan que las evidentes diferencias entre los perfiles no son las mismas mes a mes, se justifica la elección de este parámetro temporal en lugar, tal vez de un perfil por cada estación.

\subsubsection{El Nuevo Modelo Mensual (New Malargüe Monthly Model)}

Utilizando los mismos recursos que en modelo anterior, pero con una muy amplia estadística de medidas de globos radiosondas en el Observatorio Auger, a fines del 2009 el equipo de monitoreo atmosférico actualizo el modelo denominándolo New Malargüe Monthly Model (NMMM) [96].

En base a 277 perfiles correspondientes a medidas de globos realizadas en el sitio del experimento, se pudieron establecer perfiles promedios más precisos que fueron convenientemente ajustado siguiendo la estructura del modelo de Linsley. El resultado es un nuevo conjunto de 12 perfiles correspondientes uno a cada mes, estructurado en 5 capas de manera tal que las primeras 4 capas tienen límites diferentes para cada perfil y la última capa 


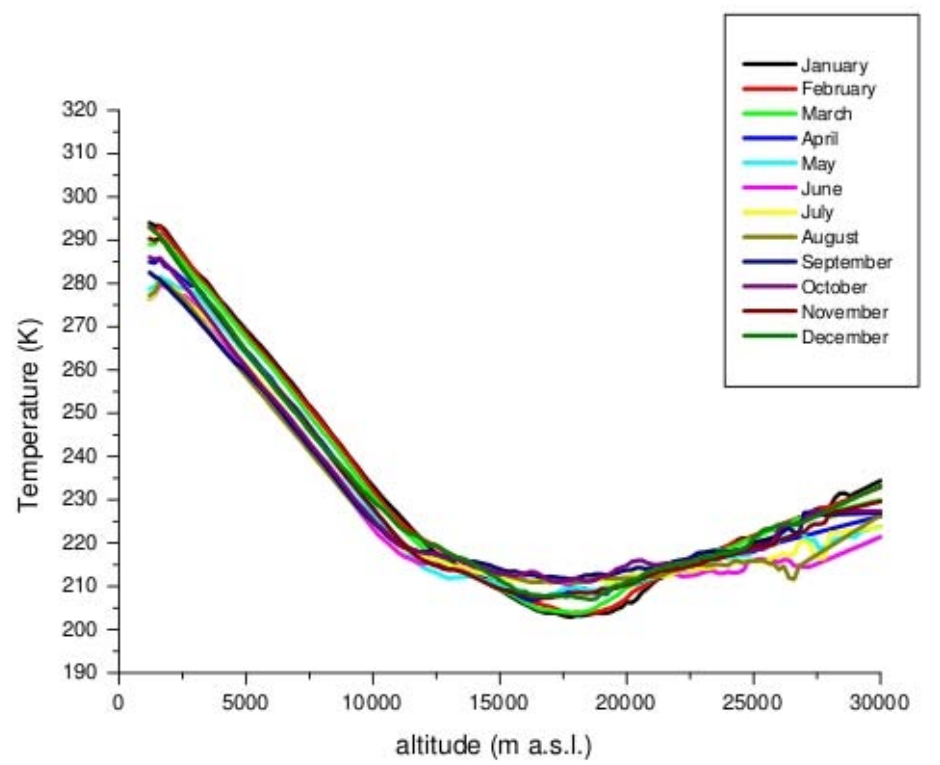

Figura 5.10: Perfiles de Temperatura del New Malargüe Monthly Model.

toma los valores de Linsley.

Como puede observarse en las figuras 5.10 y 5.11 [96], los perfiles resultantes son muy consistentes con los del $M M M$ y reproducen diferencias muy similares.

En el próximo capítulo se profundizará en los perfiles de profundidad atmosférica generados por este modelo, estableciéndose las correspondientes comparaciones con el Modelo de Linsley y el Modelo GAMMA. 


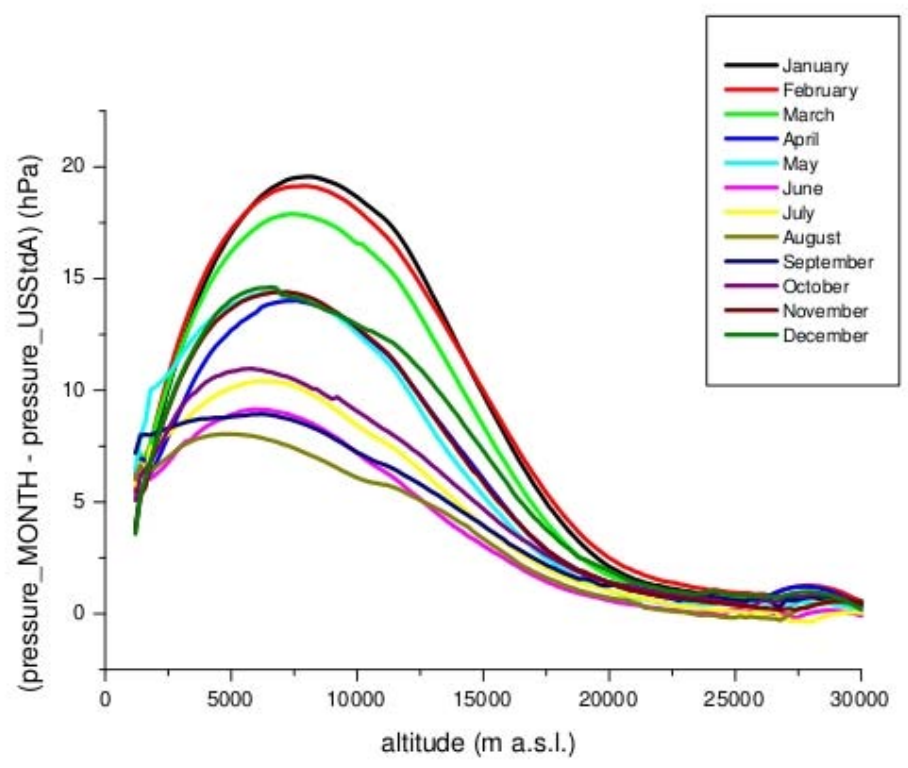

Figura 5.11: Diferencia entre los perfiles de profundidad atmosférica del New Malargüe Monthly Model y su comparación con los datos de la USStdA. 


\section{Capítulo 6}

\section{El modelo GAMMA}

En este capítulo presentaremos el modelo atmosférico GAMMA, eje central de la tesis y que ha sido difundido mediante publicaciones [103], [104] y presentaciones en diferentes encuentros y congresos [105],[106].

Este nuevo modelo es una idea original concebida con el objetivo de caracterizar el perfil de profundidad atmosférica en el sitio del Observatorio Auger, a fin de poder utilizarse en las tareas de reconstrucción de eventos y simulación de cascadas. El modelo se denomina GAMMA (acrónimo de Ground temperature Association to Multilayer isothermal Model of the Atmosphere) y su innovación consiste en la utilización de un parámetro físico de fácil registro, como lo es la temperatura al nivel del suelo; para identificar el perfil atmosférico. GAMMA consiste en una representación de la atmósfera mediante varias capas donde los perfiles de densidad y profundidad atmosférica pueden ser convenientemente representados por funciones construidas de manera de resultar continuas. Los resultados obtenidos en la utilización de GAMMA muestran una gran precisión en la representación de la atmósfera de Malargüe.

Sin más dilaciones se presentan a continuación los pasos seguidos, los detalles considerados y la justificaciones en las decisiones tomadas para la construcción del modelo GAMMA.

\subsection{Ideas motivadoras}

La dimensión física, los objetivos planteados para sus estudios y el alcance planeado para sus resultados, determinaron que el Observatorio Pierre Auger, atendiera la necesidad de contar con una precisa caracterización de la atmósfera en el sitio de observación. Esta búsqueda derivó, entre otras cosas, en la determinación precisa de los perfiles atmosféricos en Malargüe, uno de 
cuyos resultados es el Malargüe Monthly Model, anteriormente mencionado.

Como fue comentado en el capítulo previo, el Malargüe Monthly Model y su nueva versión el New Malargüe Monthly Model, consisten básicamente en agrupar las medidas de globos radio sonda de acuerdo al mes del lanzamiento y realizar un preciso ajuste con una función estructuralmente similar a la propuesta de Linsley. Esta elección de acuerdo al mes, implica algo fundamental: la caracterización de los perfiles de profundidad atmosférica de acuerdo a un parámetro temporal. Esta característica del modelo lleva a cambios discontinuos en el perfil de profundidad atmosférica que no tienen significado físico. Por ejemplo el modelo predice un perfil para un evento sucedido a las 23:55 de un 31 de Mayo, y es el mismo perfil de un evento sucedido 30 días antes, pero el perfil será diferente para el evento que suceda 10 minutos después.

Esto motivó la idea central en el desarrollo de GAMMA: un nuevo modelo que tuviera en cuenta la caracterización de los perfiles a partir de un parámetro físico. Como condición fundamental, este parámetro debía ser de fácil y rápida obtención, y de permanente disponibilidad. Considerando estas premisas se selecciona a la temperatura al nivel del suelo como este parámetro característico del modelo. En las secciones siguientes se establecerán cómo se trabajo en el desarrollo de GAMMA a partir de esta idea.

\subsection{La base de datos}

El modelo fue construido utilizando mediciones experimentales. La base de datos sobre la que se realizó el modelo GAMMA está conformada por las mediciones de globos radio sonda [107] lanzados periódicamente en el sitio del Observatorio Auger, y fue también utilizada en el ya mencionado Malargüe Monthly Model. Los globos fueron lanzados a en diferentes épocas del año, tanto durante el día como la noche, con la intención de tener un muestreo representativo de las diferentes condiciones atmosféricas en las que suceden los eventos. Estas mediciones permitieron establecer una base de datos de 277 globos que representan 277 perfiles atmosféricos entre Agosto de 2002 y Diciembre de 2008.

Los globos metereológicos utilizados eran llenados con helio y mediante el sistema de radio sondeo transmitían datos cada 8 segundos aproximadamente. La frecuencia de toma de datos permite obtener pefiles en altitud de Temperatura $T(h)$, presión $p(h)$, humedad relativa $u(h)$ y velocidad del viento $w s(h)$. Las incertezas y el rango de funcionamiento del instrumental se encuentran en el cuadro 4.1.

Con estos parámetros medidos se calcula el perfil de densidad del aire 
$\rho(h)$, mediante

$$
\rho(h)=\frac{p(h) \cdot M_{m}}{R \cdot T(h)}
$$

donde $R$ es la constante universal de los gases y $M_{m}$ es la masa molar del aire expresada en $\mathrm{g} \mathrm{mol}^{-1}$.

Particularmente, la masa molar es estimada incluyendo la contribución del vapor de agua extraida del perfil de humedad mediante la parametrización [108]:

$$
\begin{aligned}
M_{m}= & \frac{28,95949 \frac{\mathrm{g}}{\mathrm{mol}} \cdot \mathrm{vol} \%(\text { aire })}{100}+\frac{44,01 \frac{\mathrm{g}}{\mathrm{mol}} \cdot \mathrm{vol} \%\left(\mathrm{CO}_{2}\right)}{100} \\
& +\frac{18,016 \frac{\mathrm{g}}{\mathrm{mol}} \cdot \operatorname{vol} \%(\text { vapor })}{100}
\end{aligned}
$$

donde el primer término representa la contribución de aire seco y sin dióxido de carbono, el segundo la contribución del $\mathrm{CO}_{2}$, y el tercero es el aporte del vapor de agua. Este último es calculado utilizando la humedad relativa $u$,la presión $p$, y la presión de saturación $E_{s}$ mediante la expresión

$$
\operatorname{vol} \%(\text { vapor })=\left(E_{s} \cdot u\right) / p \quad .
$$

Consideremos además que la presión de saturación de vapor de agua, es función de la temperatura y se calcula mediante la fórmula de Magnus [109]

$$
E_{s}(T)= \begin{cases}6,1078 \cdot \exp \left(\frac{17,08085 \cdot T}{234,175+T}\right) & T \geq 0^{\circ} C \\ 6,10714 \cdot \exp \left(\frac{22,44294 \cdot T}{272,44+T}\right) & T<0^{\circ} C\end{cases}
$$

Por otro lado, a fin de aumentar la precisión en la determinación de los parámetros, fue incluido un preciso cálculo de la gravedad considerando la altura $h$ y la latitud $\phi$. Esto fue realizado mediante la llamada fórmula de List [108], una expresión ampliamente difundida en estudios geofísicos y meteorológicos, que representa la variación de la gravedad (medida en $\mathrm{m} / \mathrm{s}^{2}$ ) como un desarrollo en términos de la latitud y la altura (expresada en metros); siendo la misma:

$$
\begin{aligned}
g(h, \phi)= & g_{0}(\phi)-\left(3,085462 \cdot 10^{-6} 1 / s^{2}+2,27 \cdot 10^{-9} 1 / s^{2} \cdot \cos (2 \phi)\right) \cdot h \\
& +\left(7,254 \cdot 10^{-13} 1 / m s^{2}+1,0 \cdot 10^{15} 1 / m s^{2} \cdot \cos (2 \phi)\right) \cdot h^{2} \\
& -\left(1,517 \cdot 10^{19} 1 / m^{2} s^{2}+6,0 \cdot 10^{22} 1 / m^{2} s^{2} \cdot \cos (2 \phi)\right) \cdot h^{3}
\end{aligned}
$$


donde $g_{0}(\phi)$ representa la aceleración de la gravedad al nivel del mar, y puede calcularse mediante:

$$
g_{0}(\phi)=9,806160 \mathrm{~m} / \mathrm{s}^{2} \cdot\left[\begin{array}{l}
1-0,00026373 \cdot \cos (2 \phi) \\
\left.+0,0000059 \cdot \cos ^{2}(2 \phi)\right]
\end{array}\right.
$$

Los perfiles de profundidad atmosférica son obtenidos usando la aproximación

$$
\triangle X \approx \frac{\rho\left(h_{i}\right)+\rho\left(h_{i+1}\right)}{2} \cdot\left(h_{i+1}-h_{i}\right)
$$

que es válida entre dos mediciones sucesivas $(i, i+1)$.

Para cada ascenso la radio sonda adquiere datos hasta una altitud máxima $h_{b}$. Considerando la presión y aceleración gravitatoria a esa altitud, $p\left(h_{b}\right) \mathrm{y}$ $g\left(h_{b}\right)$ respectivamente, y teniendo en cuenta la aproximación

$$
p\left(h_{b}\right)=\int_{h_{b}}^{\infty} g(h) \cdot \rho(h) d h \approx g\left(h_{b}\right) \int_{h_{b}}^{\infty} \rho(h) d h
$$

es inmediato concluir que la profundidad al límite superior $h_{b}, X_{b}=X\left(h_{b}\right)$, puede estimarse a partir de

$$
X_{b} \approx \frac{p\left(h_{b}\right)}{g\left(h_{b}\right)} .
$$

El grupo de monitoreo atmosférico se encargó de procesar los datos crudos enviados de los globos radiosonda. Este trabajo consiste en determinar los valores de las medidas cada $200 \mathrm{~m}$ de altura, ya que como los globos no realizan un ascenso a velocidad constante, los datos crudos no se encuentran distribuidos uniformemente con la altura. Por ejemplo, en los primeros 500 $\mathrm{m}$ la cantidad de datos es menor que la tomada entre los 10000 m y 10500 m. De esta manera se determinan perfiles de Temperatura, presión, humedad relativa y velocidad del viento con datos cada $200 \mathrm{~m}$, se calculan los perfiles faltantes (densidad y profundidad atmosférica), y se ponen a disposición del resto de los investigadores de la colaboración.

El conjunto de medidas y los perfiles resultantes, reproducen los aspectos moleculares de la atmósfera al momento del lanzamiento del globo. El lanzamiento sistemático de globos radiosonda y el subsecuente análisis y procesamiento de sus medidas concluyen en una exhaustiva base de datos, consistente en perfiles de Temperatura, presión, humedad relativa, densidad y profundidad atmosférica que representan a diferentes atmósferas.

Como en todo análisis, es necesario un chequeo previo de los datos a ser utilizados, poniendo diferentes cortes de calidad sobre la totalidad del muestreo. En este caso realizamos dos test sobre todos los globos. El primero 
consiste en observar el perfil de lo que se ha denominado $g_{R}(h)$, resultante del cociente entre la profundidad atmosférica y la presión. Su expresión es entonces

$$
g_{R}(h)=\frac{p(h)}{X(h)}
$$

El segundo test se realiza sobre el análisis del perfil $C(h)$, obtenido a partir del cociente del perfil de la presión y el producto entre los perfiles de densidad y Temperatura. En este caso la expresión resulta

$$
C(h)=\frac{p(h)}{\rho(h) T(h)}
$$

La primera de estas funciones representa la dependencia aproximada de la gravedad con la altura, mientras que la segunda debe corresponderse con un perfil constante si el aire se comporta como un gas ideal. Ambas magnitudes fueron utilizadas para seleccionar los perfiles a ser empleados en nuestro análisis, seleccionando aquellos en los que $g_{R}(h)$ presentaba un comportamiento prácticamente lineal y simultáneamente $C(h)$ evidenciaba una leve caída de 2,88 a 2,871 en los primeros $5 \mathrm{~km}$ y luego permanecía virtualmente constante. Ejemplos paradigmáticos de esta situación pueden observarse en las figuras 6.1 y 6.2. En definitiva esta regla de selección permite disponer de una base de datos compuesta por 244 globos, es decir 244 perfiles de profundidad atmosférica.

El conjunto de perfiles resultante es agrupado de acuerdo al valor de temperatura en el suelo de cada perfil en 10 bins de $2 \mathrm{~K}$ de amplitud, cubriendo un rango de temperaturas entre 278 K y 298 K (Cuadro 6.1). Esta selección en bins implica no considerar a 32 perfiles cuya temperatura al nivel del suelo es menor que $278 \mathrm{~K}$ o mayor que $298 \mathrm{~K}$. La elección de estos límites en temperatura asegura que cada bin tenga similar estadística, situación que no se produce incluyendo estos 32 perfiles.

Los valores de temperatura en el suelo que permiten la clasificación fueron obtenidos de un ajuste lineal en el perfil de temperatura de cada globo en los primeros $5.6 \mathrm{~km}$. Esto se realizó de esta manera porque algunos de los globos no comenzaban a tomar datos sino a $200 \mathrm{~m}$ por encima del suelo. Sin embargo, los valores obtenidos para la temperatura en el suelo se correlacionaban perfectamente por los medidos en las estaciones meteorológicas del Observatorio.

El conjunto de perfiles final utilizados en la investigación consistió en 212 globos. Para cada bin en temperatura se realizó el perfil promedio de profundidad atmosférica. 

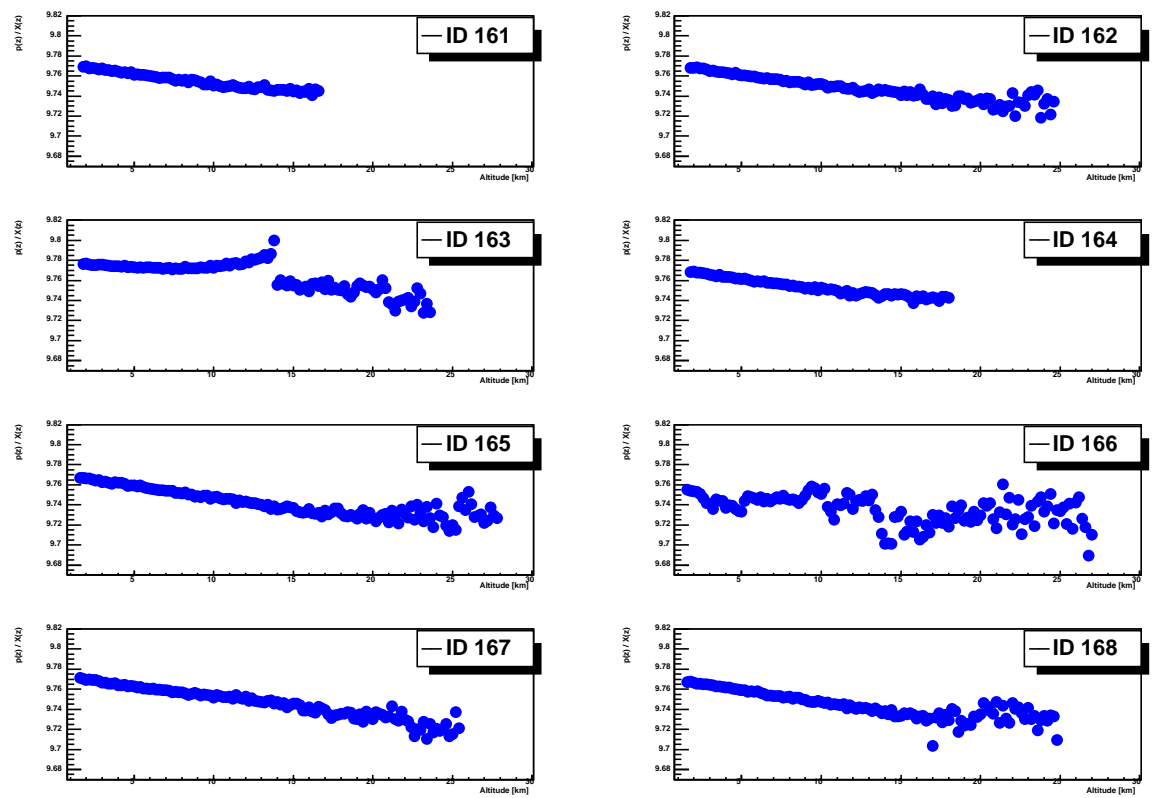

Figura 6.1: Gráfico de algunos perfiles $g_{R}(h)$. Notar que en los perfiles 163 y 166, el comportamiento de $g_{R}(h)$ se aparta de lo esperado para esta magnitud (ver texto), y por ello tales perfiles han sido descartados. 

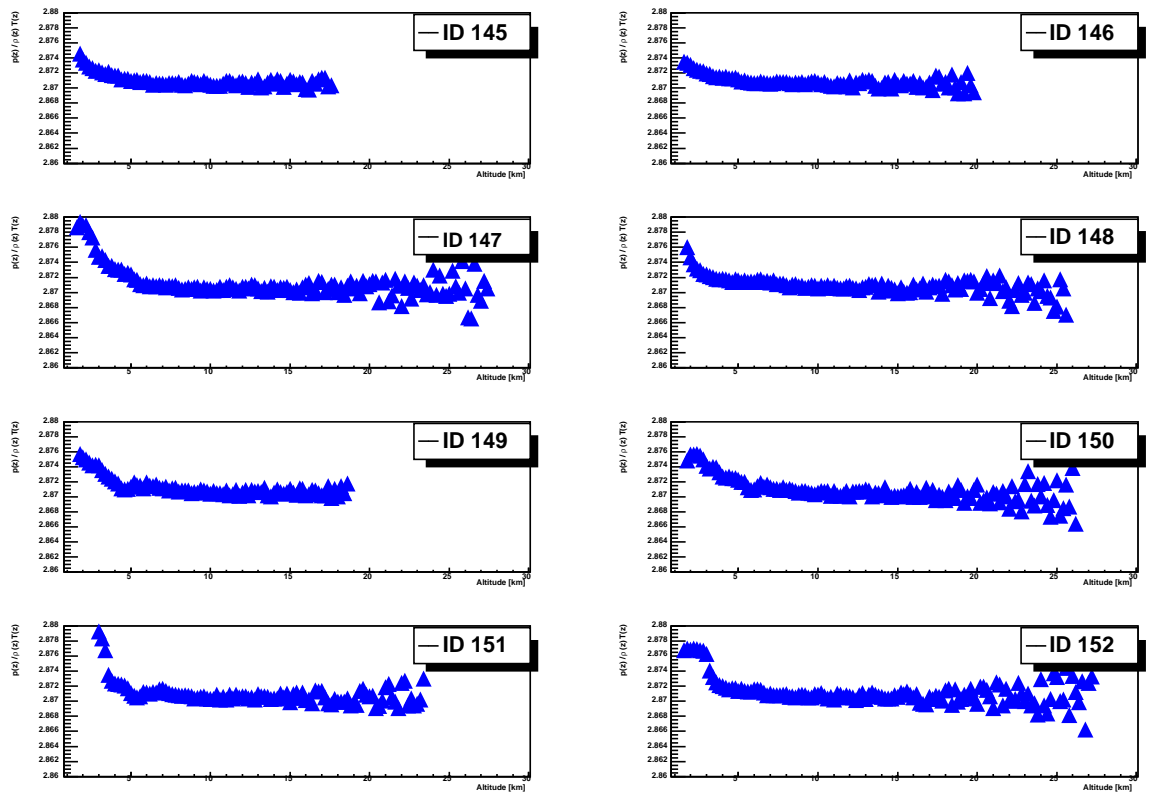

Figura 6.2: Gráfico de algunos perfiles $C(h)$. Notar que en los perfiles $14{ }^{77} y$ 152, el comportamiento de $C(h)$ se aparta de lo esperado para esta magnitud (ver texto), y por ello tales perfiles han sido descartados. 


\begin{tabular}{|c|c|c|}
\hline Bin & T rango $[\mathbf{K}]$ & Perfiles \\
\hline 1 & $278-280$ & 15 \\
\hline 2 & $280-282$ & 17 \\
\hline 3 & $282-284$ & 20 \\
\hline 4 & $284-286$ & 26 \\
\hline 5 & $286-288$ & 23 \\
\hline 6 & $288-290$ & 25 \\
\hline 7 & $290-292$ & 22 \\
\hline 8 & $292-294$ & 27 \\
\hline 9 & $294-296$ & 19 \\
\hline 10 & $296-298$ & 18 \\
\hline
\end{tabular}

Cuadro 6.1: Agrupamiento de los perfiles de acuerdo el valor de la temperatura en el suelo. La columna de la derecha contiene la cantidad de perfiles en el bin.

\subsection{La función de ajuste}

El modelo GAMMA fue construido ajustando con una función los perfiles promedio de profundidad atmosférica en cada uno de los bins. La función seleccionada replica la estructura del modelo de Linsley de 7 capas. Es decir

$$
X(h)=\left\{\begin{array}{cc}
a_{l}+b_{l} e^{-\frac{h}{c_{l}}} & h_{l-1} \leq h<h_{l} \\
a_{7}-b_{7} \frac{h}{c_{7}} & h_{6} \leq h<h_{7} \\
0 & h \geq h_{7}
\end{array}\right.
$$

donde los límtes $h_{l}$ elegidos para las capas se muestran en el Cuadro 6.2.

\begin{tabular}{|c|c|c|}
\hline Capa & Lím inferior $[\mathrm{km}]$ & Lím superior $[\mathrm{km}]$ \\
\hline $\mathbf{1}$ & 0 & 3.5 \\
\hline $\mathbf{2}$ & 3.5 & 8.5 \\
\hline $\mathbf{3}$ & 8.5 & 16 \\
\hline $\mathbf{4}$ & 16 & 24 \\
\hline $\mathbf{5}$ & 24 & 40 \\
\hline $\mathbf{6}$ & 40 & 100 \\
\hline $\mathbf{7}$ & 100 & 112.8 \\
\hline
\end{tabular}

Cuadro 6.2: Límites de las capas atmosféricas del modelo GAMMA

La altura máxima que alcanzan los globos es alrededor de $26 \mathrm{~km}$, de manera tal que el ajuste de los perfiles fue realizado sólo en las primeras 5 
capas. A los $26 \mathrm{~km}$ el valor de la profundidad atmosférica es aproximadamente de $20 \mathrm{~g} / \mathrm{cm}^{2}$, con lo cual el rango más importante de estudio para rayos cósmicos estaba cubierto. Esta última afirmación se basa en que las cascadas de partículas poseen un significante desarrollo después de los $100 \mathrm{~g} / \mathrm{cm}^{2}$. Para alturas mayores, los coeficientes $a_{l}, b_{l}$ y $c_{l}$ son idénticos a los correspondientes a las dos últimas capas de la función de Linsley de 5 capas (Cuadro 5.2).

El ajuste se realiza con particulares condiciones detalladas a continuación.

- Continuidad: La función $X(h)$ y su derivada $\rho(h)$ deben ser continuas entre todas las capas. Incluye también el empalme con las capas superiores de la función de Linsley.

- Condiciones de Contorno: La función $X(h)$ y su derivada $\rho(h)$ se fijan a los valores de $39.8 \mathrm{~km}$ provenientes de los valores de Linsley de 5 capas.

Con estas consideraciones el ajuste tiene 5 parámetros libres, y por simplicidad en este modelo hemos elegido que sean $\operatorname{los} c_{l}(l=1,2,3,4,5)$. Con ellos pueden obtenerse los valores del resto de los parámetros $a_{l}$ y $b_{l}(l=1,2,3,4,5)$ mediante expresiones que se encuentran en el Apéndice A.

\subsection{Resultados de los ajustes}

Con la función definida y las condiciones prefijadas se realiza entonces los ajustes sobre cada uno de los perfiles promedios del bin, arrojando los valores $c_{1}, c_{2}, c_{3}, c_{4}$ y $c_{5}$ de la expresión 6.12 . Todo los ajustes fueron realizado mediante la rutina de minimización MINUIT, incluida en el software ROOT [56]. Como ejemplo de los buena precisión obtenida en los mismos pueden verse las figuras [6.3] y [6.4].

Prosiguiendo con la construcción del modelo, el siguiente paso fue el de obtener el comportamiento de $\operatorname{los} c_{1}, c_{2}, c_{3}, c_{4}$ y $c_{5}$ como funciones de la temperatura en el suelo. Con ese sentido es que se graficaron los parámetros obtenidos (Figura 6.5). Los valores obtenidos, y la búsqueda de una descripción simplificada, llevaron a suponer un comportamiento lineal con la temperatura en el suelo para cada uno de los $c_{l}$, que derivó en un ajuste lineal para esos puntos.

Los resultados de este último ajuste figuran en la Tabla 6.3.

Una vez ajustados los coeficientes estamos en condiciones entonces de conocer el perfil de profundidad atmosférico en cualquier momento del día si conocemos el valor de la temperatura en el suelo y si ésta se encuentra entre $T_{M I N}=278 \mathrm{~K}$ y $T_{M A X}=298 \mathrm{~K}$. La obtención de los diferentes perfiles 

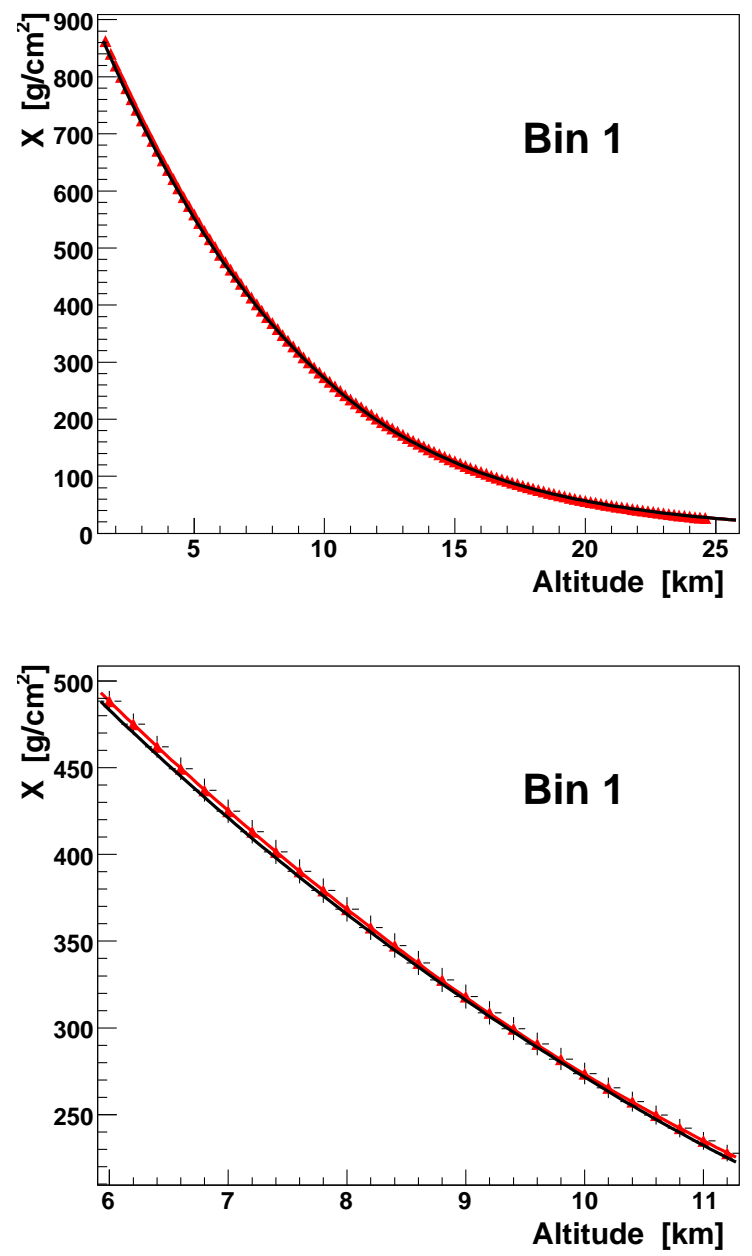

Figura 6.3: Valores de $X(h)$ correspondiente al Bin 1 y su ajuste de acuerdo a la función 6.12. Se observa además un efecto de zoom para apreciar el ajuste. La línea punteada representa la parametrización correspondiente al Modelo de Linsley de 5 capas. 

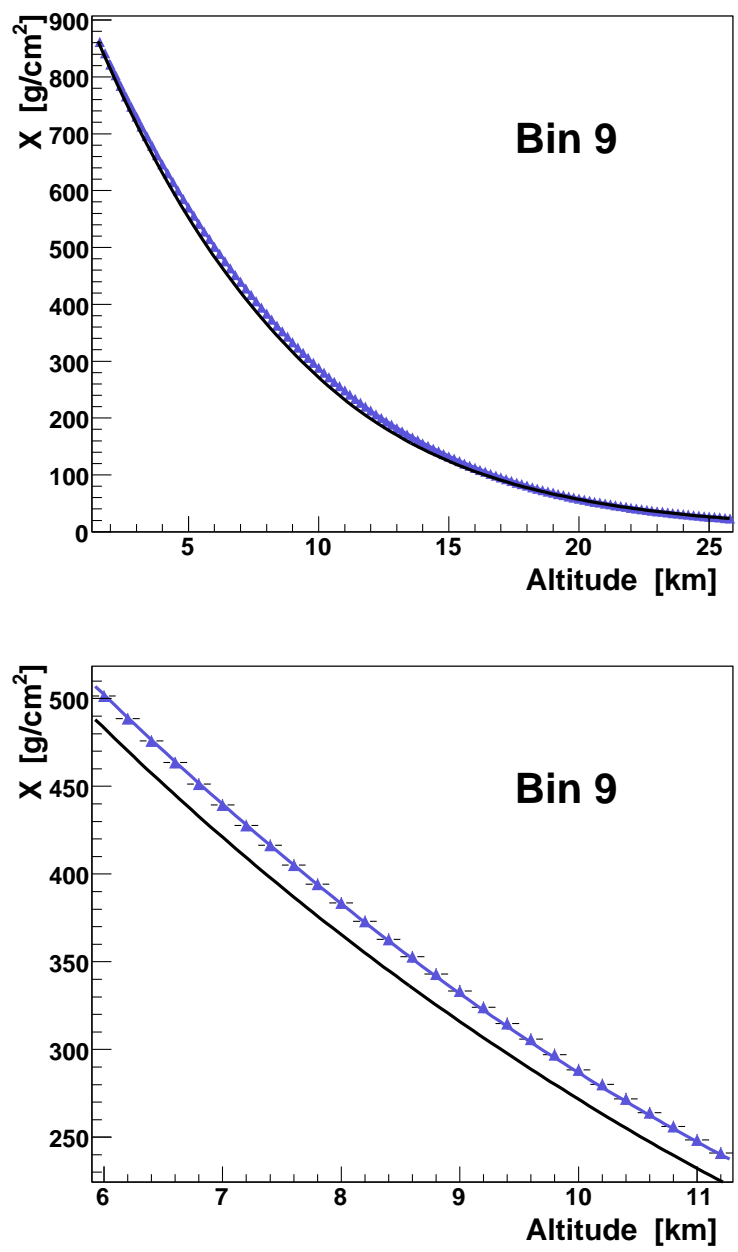

Figura 6.4: Valores de $X(h)$ correspondiente al Bin 9 y su ajuste de acuerdo a la función 6.12. Se observa además un efecto de zoom para apreciar el ajuste. La línea punteada representa la parametrización correspondiente al Modelo de Linsley de 5 capas. 

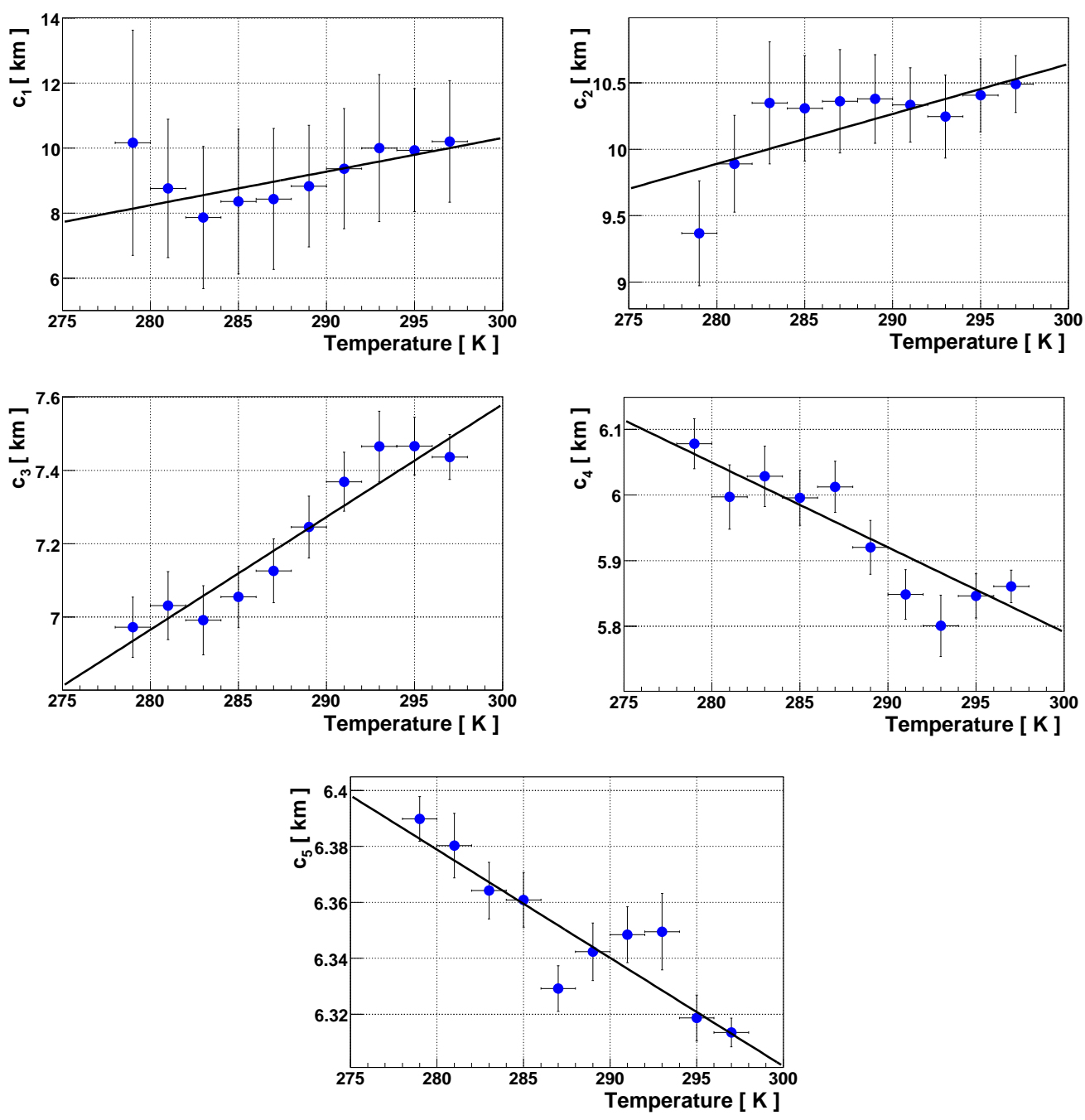

Figura 6.5: Valores de $c_{1}, c_{2}, c_{3}, c_{4}, c_{5}$, obtenidos en cada ajuste sobre diferentes bins de Temperatura en el Suelo. Se observa además un ajuste lineal sobre esos valores. 


\begin{tabular}{|c|c|c|}
\hline Capa (l) & $N_{l}[\mathrm{~km}]$ & $M_{l}[\mathrm{~km} / \mathrm{K}]$ \\
\hline $\mathbf{1}$ & -20.716 & 0.1034 \\
$\mathbf{2}$ & -0.626 & 0.0376 \\
$\mathbf{3}$ & -1.64 & 0.0307 \\
$\mathbf{4}$ & 9.668 & -0.0129 \\
$\mathbf{5}$ & 7.463 & -0.00038 \\
\hline
\end{tabular}

Cuadro 6.3: Valores de la parametrización lineal $c_{l}\left(T_{G R O U N D}\right)=$ $M_{l} T_{G R O U N D}+N_{l}$.

de profundidad atmosférica acorde al valor de la temperatura en el suelo, consiste simplemente en obtener los valores de $c_{l}$ utilizando la expresión

$$
c_{l}\left(T_{G R O U N D}\right)=M_{l} T_{G R O U N D}+N_{l} \quad(l=1, \ldots, 5),
$$

donde los valores de $M_{l}$ y $N_{l}$ se obtienen de la Tabla 6.3 y $T_{G R O U N D}$ es el valor de la temperatura en el suelo. Los parámetros $a_{l}, b_{l}(l=1,2,3,4,5)$ se obtienen a partir de los $c_{l}\left(T_{G R O U N D}\right)$ mediante las expresiones que se enceuntran en el Apéndice A. Los parámetros restantes $a_{l}, b_{l}$ y $c_{l}(l=6,7)$ se obtienen de la parametrización de Linsley (Cuadro 5.2), permitiendo construir la función

$$
X_{\text {GAMMA }}=\left\{\begin{array}{cc}
a_{l}+b_{l} e^{-\frac{h}{c_{l}}} & h_{l-1} \leq h<h_{l} \\
a_{7}-b_{7} \frac{h}{c_{7}} & h_{6} \leq h<h_{\text {lim }} \\
0 & h \geq h_{\text {lim }}
\end{array} \quad(l=1, \ldots, 6)\right.
$$

para cada perfil.

\subsection{Verificando el Modelo GAMMA}

Con el objetivo de analizar la validez de los resultados alcanzados, se procedió a realizar una serie de comprobaciones. Básicamente consistieron en analizar las incertezas que surgen del uso del modelo, y la comparación con los resultados mostrados por los modelos de Linsley y Malargüe Monthly Model.

\subsubsection{Estudio de Residuos}

El primer análisis consitió en tomar el valor de la temperatura en el suelo para cada perfil experimental de profundidad atmosférica $X_{E X P}(h)$ y 
calcular el perfil $X_{\text {GAMMA }}(h)$ que predice el modelo GAMMA para ese valor de temperatura en el suelo.

Definiendo el residuo como

$$
\operatorname{Residuo}(h)=X_{E X P}(h)-X_{G A M M A}(h) \quad,
$$

se comparó primero el comportamiento de los residuos como función de la altura manteniendo la separación en los bins originales en Temperatura (Figuras [6.6] y [6.7]). Esto permitió comprobar el ajuste realizado en cada bin, concluyendo que el ajuste es preciso en todos los rangos de Temperatura estudiados. Los valores medios de los residuos para cada altura se encontraban todos comprendidos en el rango $\sim 2 \mathrm{~g} / \mathrm{cm}^{2} \pm 4 \mathrm{~g} / \mathrm{cm}^{2}(\sim 0.7 \% \pm 1.4 \%)$.
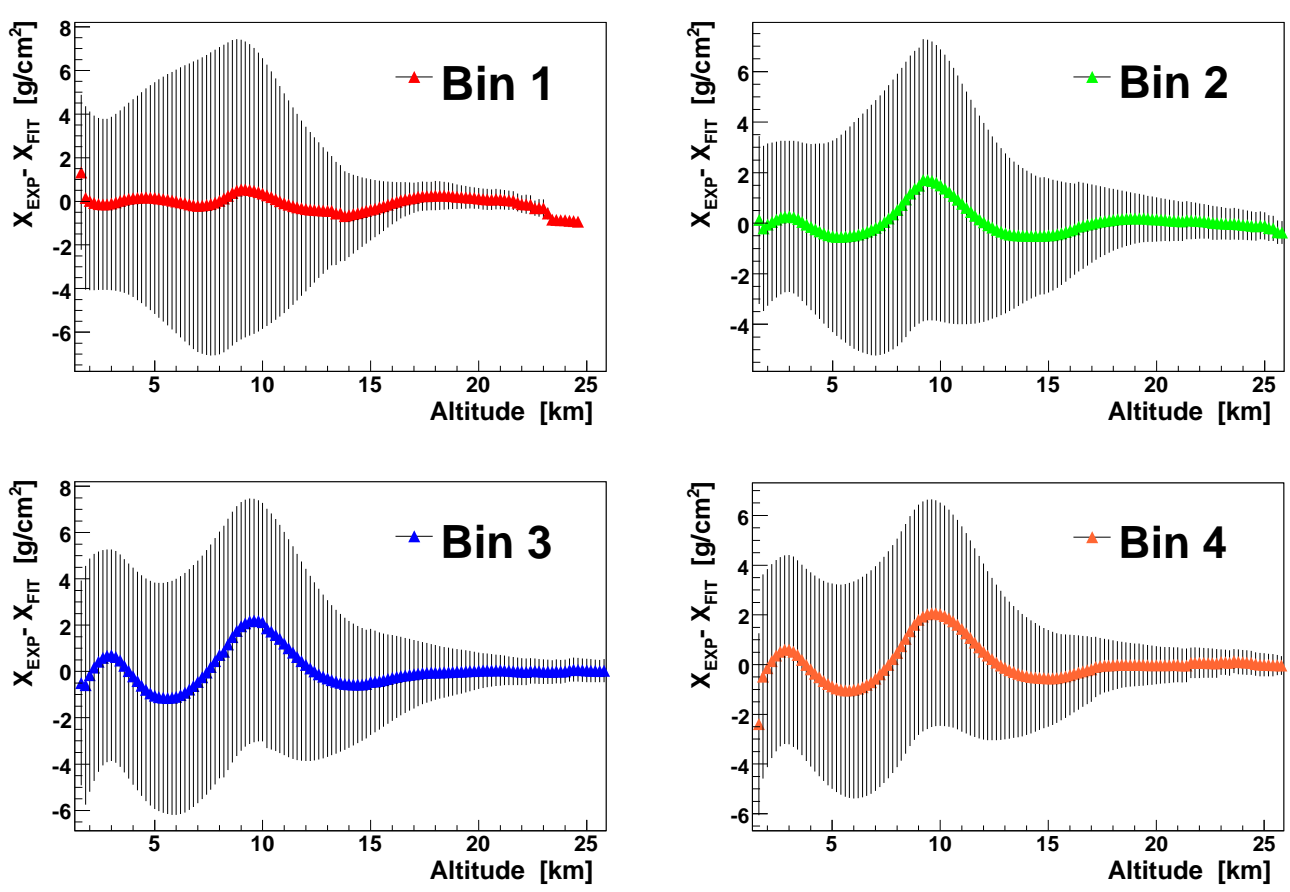

Figura 6.6: Residuos como función de la altura para el Modelo GAMMA (Bins 1, 2,3 y 4$)$.

Para tener una idea completa sobre las diferencias en las predicciones del modelo GAMMA, se realizó también el cálculo de los residuos en función de la altura sin discriminar en bins de temperatura, es decir observando la diferencia entre la predicción del modelo GAMMA y los datos de cada uno de los 212 perfiles con Temperatura en el suelo entre 278 K y 298 K. Estos resultados están reflejados en la Figura [6.8] (cada pixel del gráfico representa 

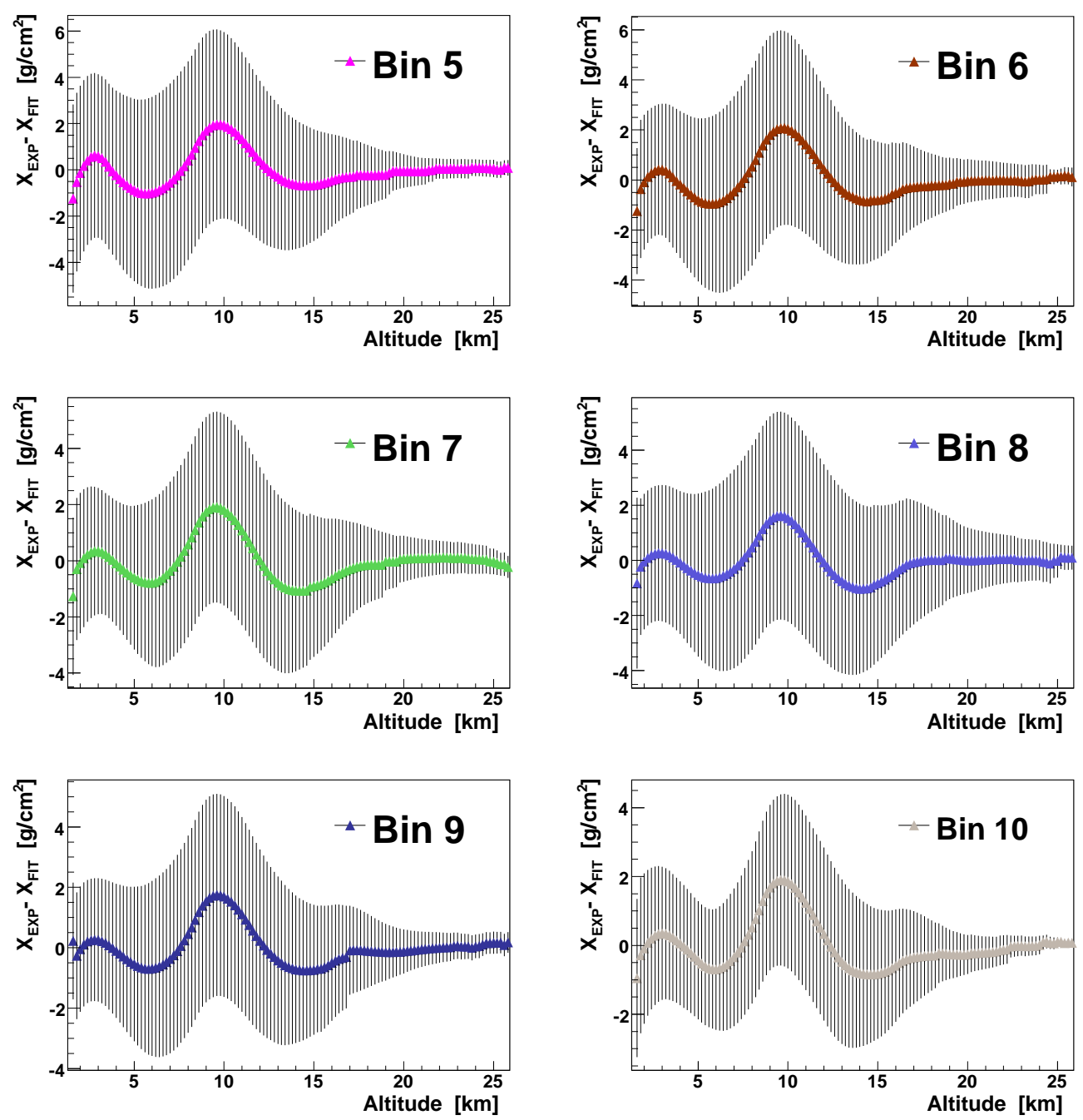

Figura 6.7: Residuos como función de la altura para el Modelo GAMMA (Bins 5, 6, 7, 8, 9 y 10). 


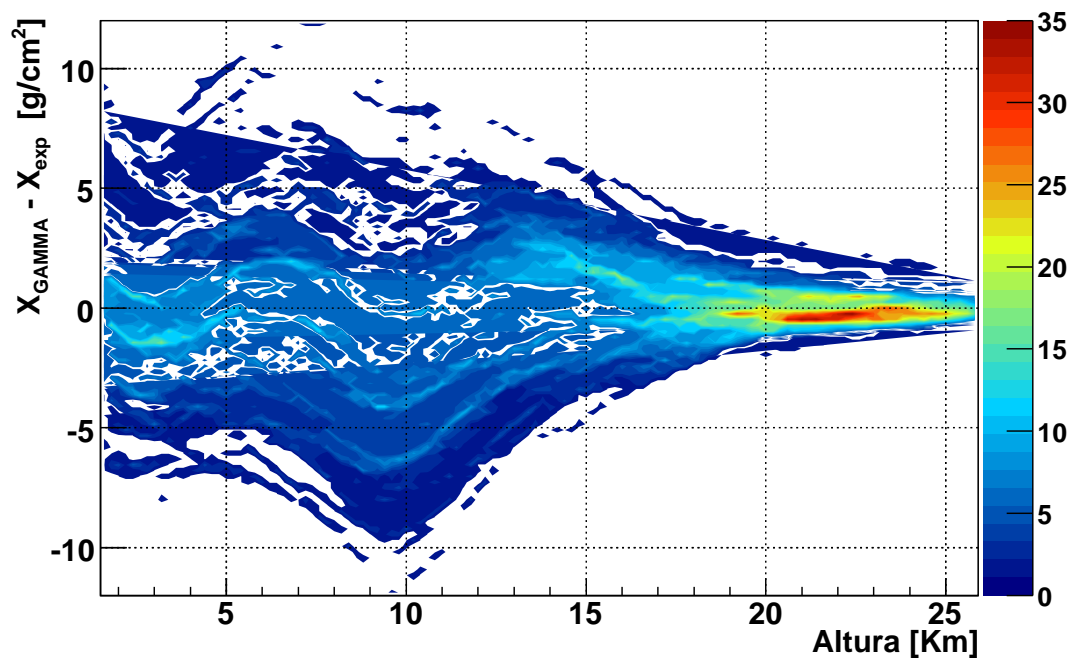

Figura 6.8: Residuos como función de la altura para el Modelo GAMMA, realizado sobre todo el conjunto de datos.

una variación de $0.25 \mathrm{~g} / \mathrm{cm}^{2}$ y $200 \mathrm{~m}$ ). Este grafíco reproduce en forma similar la estructura de residuos observada en las figuras [6.6] y [6.7], cuando estaba separado en bines de Temperatura.

Para comprobar si la diferencia entre las predicciones era mayor para algunos valores de Temperatura en el suelo, se procedió a graficarla para todo el conjunto de datos (Figura 6.9) (cada pixel del gráfico representa una variación de $0.25 \mathrm{~g} / \mathrm{cm}^{2}$ y $0.5 \mathrm{~K}$ ). Claramente se observa una dispersión relativamente homogénea, indicando que no hay temperaturas preferenciales para el Modelo GAMMA.

Por último se compararon las predicciones realizadas por el Modelo de Linsley, el Malargüe Monthly Model y el Modelo GAMMA para cada perfil de profundidad atmosférica (sin separación en bins), realizando en cada caso la diferencia entre la predicción y el dato experimental. Estas comparaciones se observan en la figura 6.10. Se observa que la incerteza promedio del modelo GAMMA es aproximadamente $2 \mathrm{~g} / \mathrm{cm}^{2} \pm 4 \mathrm{~g} / \mathrm{cm}^{2}$ (Figura 6.10 arriba-izquierda) y es consistente con las incertezas de la predicción del New Malargüe Monthly Model (Figura 6.10 arriba-derecha). Como era esperable, los residuos resultantes de la comparación con el modelo de Linsley mantienen valores significativamente superiores (Figura 6.10 abajo). 


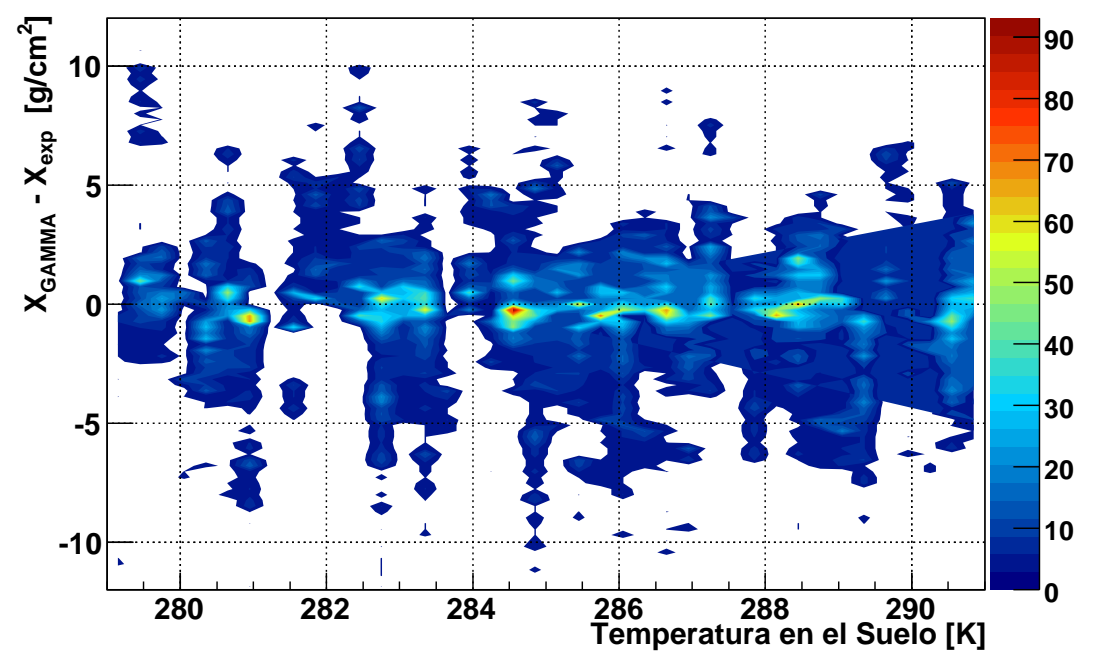

Figura 6.9: Residuos como función de la Temperatura en el suelo para el Modelo GAMMA, realizado sobre todo el conjunto de datos.

\subsubsection{Otra comparación con Linsley}

Antes de dar paso a la anunciada nueva comparación con el modelo de Linsley, justifiquemos el por qué esa relativa insistencia en contrastar ambos modelos. Uno pensaría que, teniendo en cuenta que el modelo de Linsley surge de parametrizar los datos de la USStdA, no tiene sentido compararla con un modelo obtenido a partir de medidas realizadas en Malargüe. Sin embargo, rescatemos algunos puntos que tal vez se han perdido de vista o que no estaban aún explicitados en este trabajo.

Primero hay que recordar que el modelo de Linsley fue concebido como un modelo global. Es decir como un modelo que refiere a un perfil promedio de profundidad atmosférica en un sitio que se encuentra a latitud media. Esto hace que el modelo de Linsley sea mayoritariamente la primera opción en el caso de que no hubieran datos atmosféricos locales. Recordemos justamente que la atmósfera promedio que representa fue calculada en base a medidas realizadas no sólo en Estados Unidos sino que incluye diversos sitios del planeta. Estas consideraciones no excluyen al sitio de Malargüe, ya que el mismo se encuentra precisamente en una zona de latitud media.

Otra de las razones por las cuales es razonable comparar con Linsley, es el tema de los software de simulaciones de cascadas. Los programas más utilizados, AIRES y CORSIKA, utilizaron desde sus inicios a el perfil provisto por el modelo de Linsley de 5 capas. De manera reciente se han incluido atmósfe- 

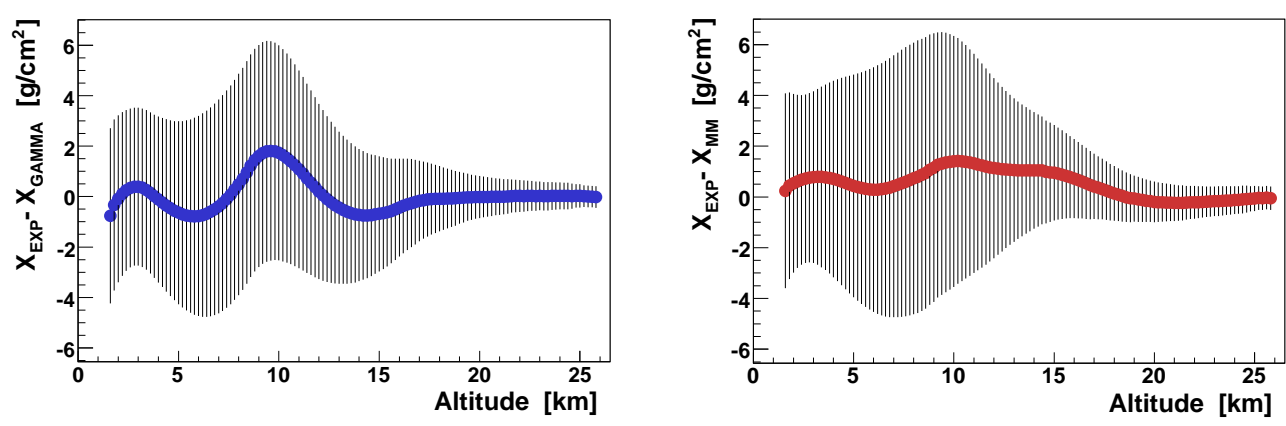

(a) Modelo GAMMA - Base de Datos origi- (b) Modelo New Malargüe Monthly Model nal.

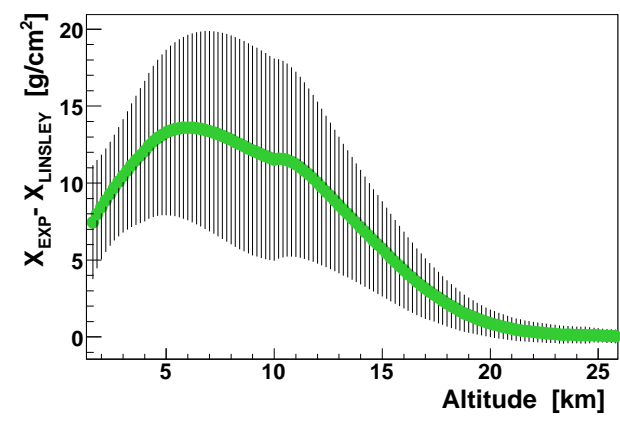

(c) Modelo de Linsley

Figura 6.10: Diferencias entre la predicción del modelo y el dato experimental. Realizado sobre todo el conjunto de datos y para los diferentes modelos: GAMMA Model (izq), Malargüe Monthly Model (der) y Linsley Model (centro). 
ras que representan otros sitios, concretamente el NMMM ya se encuentra disponible para el uso en CORSIKA y pronto lo estará en AIRES.

Por último aparece el desarrollo del New Malargüe Monthly Model. Es que este modelo, como queda reflejado en la figura 5.11, comparó sus predicciones con la del modelo de Linsley. Con lo cual, un nuevo modelo (GAMMA) construido con los mismos datos del mismo sitio debería también mantener las diferencias encontradas por el anterior (NMMM).

Habiendo reafirmado los motivos, comentaremos en qué consistió la nueva comparación. Se estudiaron los perfiles provistos por el modelo GAMMA para diversas temperaturas al nivel del suelo, comparándolos con el perfil provisto por el modelo de Linsley. Para observar si existía una tendencia vinculada a la temperatura incluso se observaron perfiles correspondientes a valores de temperatura al nivel del suelo por fuera del rango de validez establecido inicialmente por el modelo. Concretamente se estudiaron 11 perfiles correspondientes a temperaturas entre $258 \mathrm{~K}$ y $308 \mathrm{~K}$ espaciados cada $5 \mathrm{~K}$. En la figura 6.11 puede verse parte de este estudio.

Mas allá de que la comparación involucra a dos modelos construidos sobre base de datos diferentes, esta última figura promueve cuatro lecturas: (i) una señal de advertencia cuando se usa el modelo de Linsley, (ii) la diferencia entre el perfil de Linsley y los modelos GAMMA y NMMM conservan la misma estructura funcional, (iii) la diferencia promedio con el modelo de Linsley aumenta con los perfiles correspondientes a mayores valores de temperatura al nivel del suelo, y (iv) nuevamente identificar la validación de la temperatura al nivel del suelo como un buen parámetro para caracterizar los perfiles de profundidad atmosférica.

\subsubsection{El uso en el Observatorio Auger: más sobre las predicciones del Modelo GAMMA y del Modelo NMMM}

Habiendo ya establecido que los dos modelos desarrollados para el Observatorio Auger predicen mejor el perfil atmosférico que el modelo Linsley; otro de los análisis que se hicieron correspondió a la comparación entre las predicciones del Modelo GAMMA y del Malargüe Monthly Model para medidas en días críticos en relación al modelo.

Concretamente se analizaron las predicciones correspondientes a mediciones realizadas con valor de temperaturas en el suelo cercanos a los valores mínimo y máximo definidos para el modelo GAMMA. Estas predicciones fueron contrastadas con las resultantes de inferir los perfiles a partir del $M a$ largüe Monthly Model. 


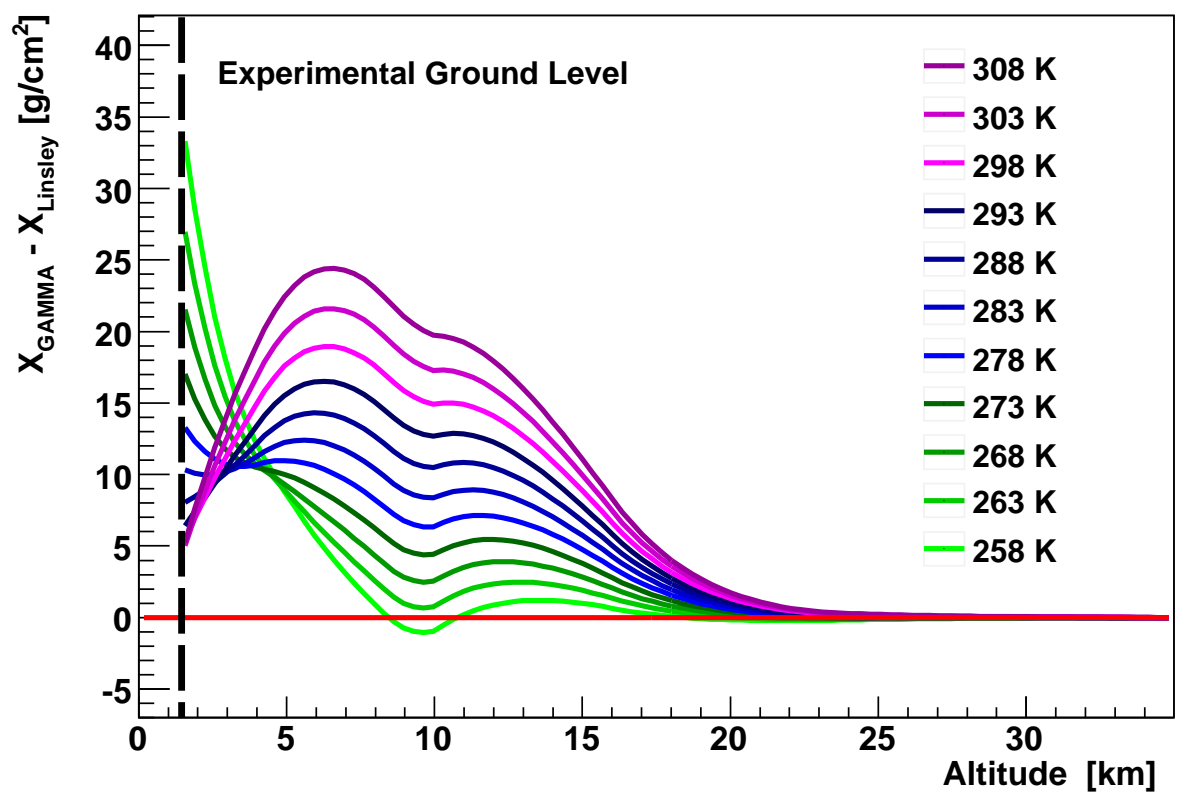

Figura 6.11: Diferencias entre el perfil de profundidad atmosférica que predice el modelo de Linsley de 5 capas y varios perfiles obtenidos del modelo GAMMA con diferentes temperaturas al nivel del suelo. Con el objetivo de observar la tendencia que parece marcar los perfiles de GAMMA a medida que se aumenta la temperatura, se incluyeron perfiles calculados por fuera del rango de validez del modelo (casos $308 \mathrm{~K}, 303 \mathrm{~K}, 273 \mathrm{~K}, 268 \mathrm{~K}, 263 \mathrm{~K}$ y 258 $K)$. La línea punteada indica el nivel del suelo en el sitio del experimento. 
Las comparaciones se realizaron sobre mediciones de perfiles con las características de temperatura en el suelo y mes (los dos datos necesarios para calcular en cada modelo) que se observan en el Cuadro 6.4.

\begin{tabular}{|c|c|c|}
\hline Característica & Temperatura & Mes \\
\hline Frío & $278.1 \mathrm{~K}$ & Agosto \\
Frío & $278.0 \mathrm{~K}$ & Septiembre \\
Frío & $277.9 \mathrm{~K}$ & Mayo \\
Caluroso & $298.7 \mathrm{~K}$ & Febrero \\
Caluroso & $300.2 \mathrm{~K}$ & Marzo \\
Caluroso & $297.9 \mathrm{~K}$ & Marzo \\
\hline
\end{tabular}

Cuadro 6.4: Datos de mediciones usadas en la comparación del modelo GAMMA y Malargüe Monthly Model en días críticos.

Las predicciones resultantes pueden observarse en la Figura 6.12, donde el modelo GAMMA está identificado con azul para los días considerados fríos y en rojo para los días estimados como calurosos. En verde se encuentra la estimación del Malargüe Monthly Model.

Se puede observar que en todos los casos las diferencias entre los perfiles experimentales y las predicciones de los modelos no son completamente despreciables, y resultan en promedio aproximadamente del mismo orden de magnitud para ambos. Pero vale la pena remarcar que se observa asimismo que la predicción del modelo GAMMA resulta levemente mejor en algunos rangos de alturas de estos casos particulares, existiendo situaciones en donde la desviación del modelo NMMM alcanza valores que exceden el doble de la correspondiente desviación del modelo GAMMA a la misma altitud (ver figura 6.10).

\subsubsection{Chequeo de consistencia}

Otro de los chequeos realizados para probar la validez del modelo desarrollado, consistió en probar las predicciones de GAMMA con una nueva base de datos de globos radiosonda. Efectivamente, transcurrido un tiempo posterior a la construcción del modelo (tiempo durante el cual el grupo de monitoreo atmosférico del Observatorio Auger continuó el lanzamiento de globos), se confeccionó una nueva base de datos con mediciones realizadas en Malargüe.

Esta base de datos independiente estaba compuesta por 43 perfiles obtenidos en el período comprendido entre enero de 2009 y agosto de 2010. Los perfiles fueron construidos de igual manera que los anteriores (ver sección $6.1)$. 

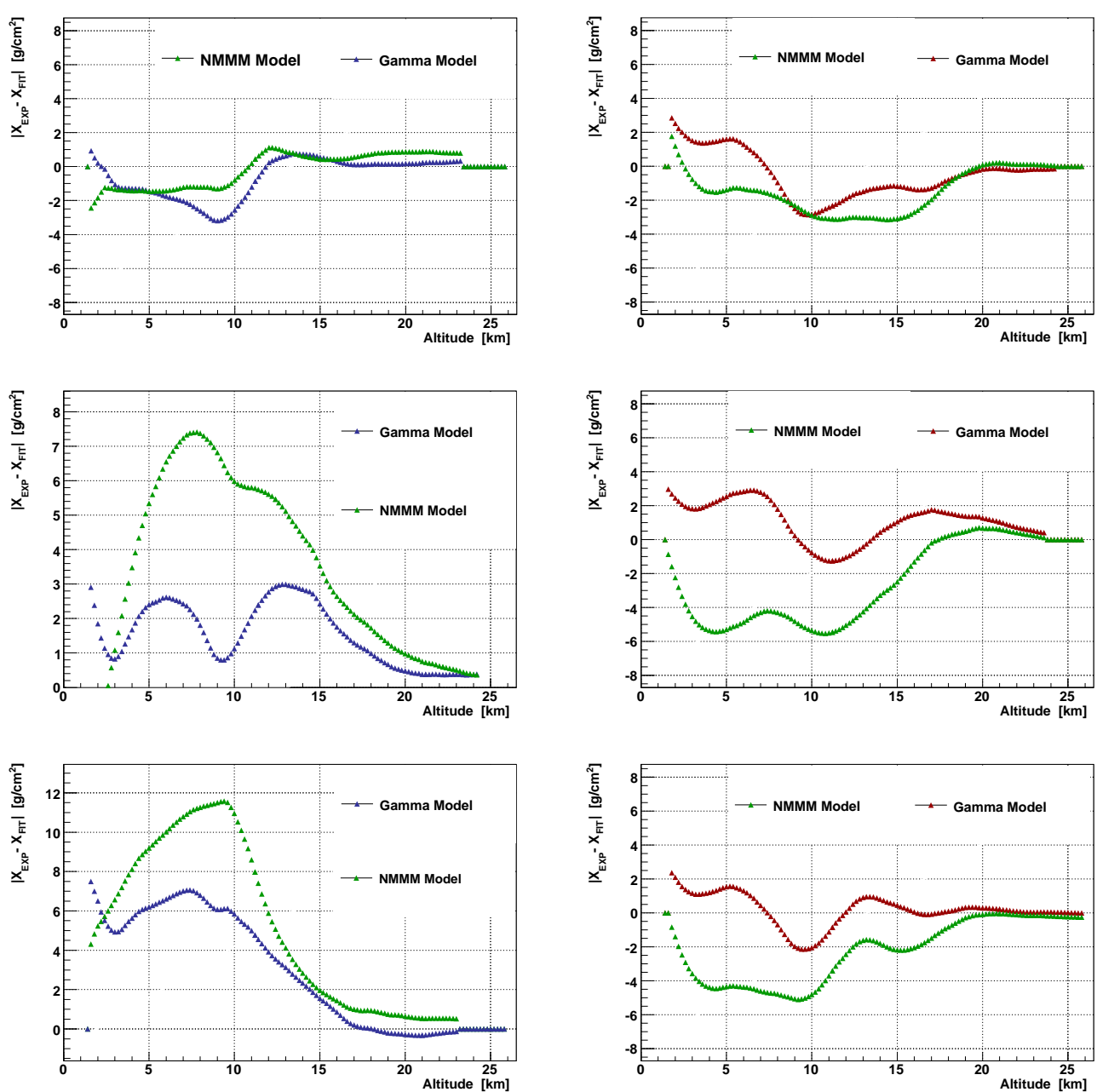

Figura 6.12: Diferencias entre las predicciones de los modelos GAMMA y Malargüe Monthly Model y los datos experimentales de días particulares. A la izquierda se encuentran para días fríos (temperatura en el suelo de 278.1 $K, 278 K$ y $277.9 \mathrm{~K}$ respectivamente) y a la derecha para días considerados como calurosos (temperatura en el suelo $298.7 \mathrm{~K}$, 300.2 K y $297.9 \mathrm{~K}$ respectivamente). 
Se procedió a analizar los residuos que se producían en la comparación con el modelo GAMMA. Es decir, se tomaba cada perfil, se observaba el valor de su temperatura en el suelo, se calculaba la predicción de GAMMA para esa temperatura, y se calculaba las diferencia entre esa predicción y cada valor del perfil. Todas esas diferencias se encuentran graficadas en la figura 6.13 .

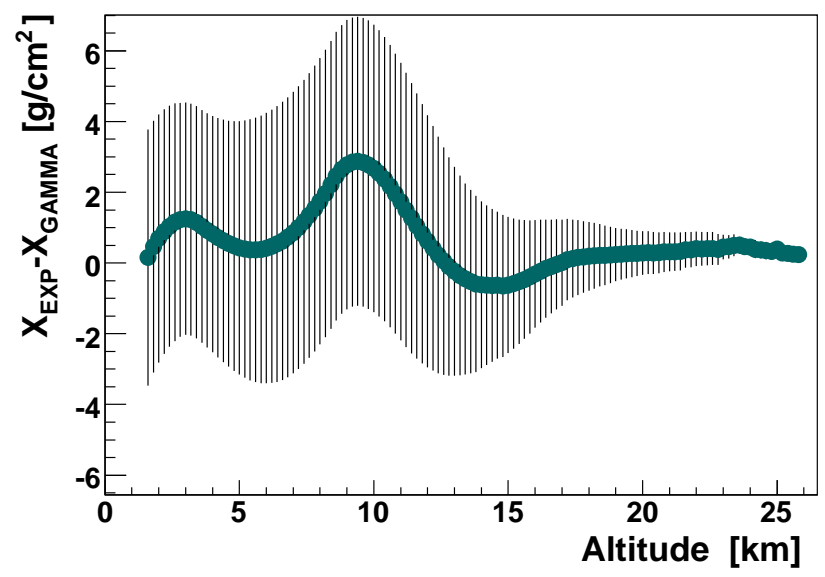

Figura 6.13: Perfil de residuos promedio resultantes de la comparación entre las predicciones de GAMMA y la nueva base de datos independiente con perfiles colectados entre Enero 2009 y Agosto 2010.

De esta figura, se desprende que los valores observados para los residuos promemedio son del orden de $3 \mathrm{~g} / \mathrm{cm}^{2} \pm 4 \mathrm{~g} / \mathrm{cm}^{2}$, cifras levemente mayor que en el caso original $\left(2 \mathrm{~g} / \mathrm{cm}^{2} \pm 4 \mathrm{~g} / \mathrm{cm}^{2}\right)$.

El haber podido reproducir prácticamente los mismos valores de incertezas con una base de datos independiente confirma la validez de GAMMA como modelo para reproducir los perfiles de profundidad atmosférica en Malargüe. No solo verifica que los valores de $\mathrm{N}_{l}$ y $\mathrm{M}_{l}$ que determinan los coeficientes de las parametrizaciones son válidos; sino que es válida la idea central del modelo: se pueden caracterizar los perfiles de profundidad atmosférica mediante el valor de la temperatura al nivel del suelo.

\subsection{Rango de validez de GAMMA}

Como se estableció previamente, el rango de temperaturas en el suelo utilizados para desarrollar el modelo GAMMA corresponde al intervalo [278 K, 298 K]. La razón para excluir otros perfiles por fuera de ese rango, estaba 
fundamentada en la baja estadística proveniente de la base de datos. Las posibilidades de ampliar el número o el tamaño de los bins en temperatura fueron probadas y no eran satisfactorias, ya que inducían incertezas mayores, además de originar importantes diferencias en la cantidad de perfiles que correspondían a cada bin.

Sin embargo, esto no implica que la utilización del modelo no fuera posible por fuera del rango de temperaturas. Se procedió entonces a comparar la predicción del modelo GAMMA (con los coeficientes obtenidos a partir de los 212 perfiles entre $278 \mathrm{~K}$ y $298 \mathrm{~K}$ ) con los valores medidos para perfiles con temperatura en el suelo fuera del rango [278 K, $298 \mathrm{~K}]$. Se encuentran un total de 19 perfiles por debajo de $278 \mathrm{~K}$, comprendidos entre $269 \mathrm{~K}$ y 278 K; y 13 perfiles entre 298 K y 304 K. Ejemplos de las diferencias entre las predicciones y los datos pueden encontrarse en la Figura 6.14.
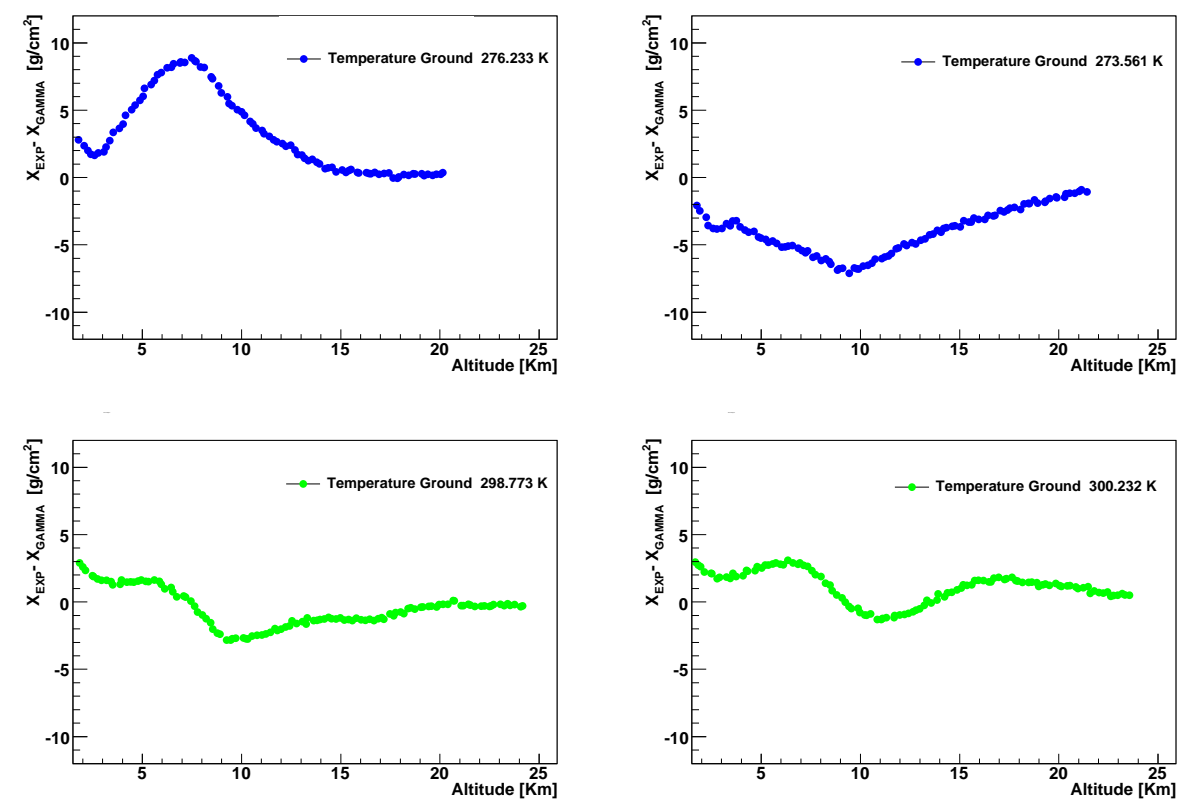

Figura 6.14: Diferencias entre la predicción del modelo GAMMA y el dato experimental para los perfiles con valor de temperatura al nivel del suelo menor a $278 \mathrm{~K}$ (izq) y mayor a $298 \mathrm{~K}$ (der). Estos perfiles no fueron utilizados en el desarrollo del Modelo GAMMA.

Cuando se representan conjuntamente las diferencias de todos los perfiles, observamos que aquellos cuyas temperaturas en el suelo correspondan a valores por debajo de $278 \mathrm{~K}$ poseen una mayor dispersión (Figura 6.15). Se estima que esto pueda ser debido a la cercanía de esos valores con el punto de congelación del agua. El no poseer buena estadística en esos casos, nos 
impide ahondar más profundamente en el estudio de esa región.

No sucede lo mismo para aquellos 13 perfiles cuyo valor de temperatura en el suelo se encuentra por encima de 298 K. Estos perfiles son reproducidos con muy buena precisión por el modelo GAMMA (Figura 6.16) y reproducen prácticamente el mismo nivel de incerteza $\left(2 \mathrm{~g} / \mathrm{cm}^{2} \pm 4 \mathrm{~g} / \mathrm{cm}^{2}\right)$.

Debido a este última verificación - de poder utilizar el modelo con coeficientes obtenidos para $[278 \mathrm{~K}, 298 \mathrm{~K}]$ con valores de temperatura en el suelo entre 298 K y 304 K manteniendo el nivel de incertezas, se decide ampliar el rango de validez del modelo GAMMA hasta Temperaturas en el suelo entre 278 K y $304 \mathrm{~K}$. Si bien las temperaturas al nivel del suelo en el sitio del Observatorio Auger pueden encontrarse eventualmente fuera de ese rango, lamentablemente no se poseen medidas de esos perfiles, es decir no hubo lanzamientos de globos con radio sondas en esas condiciones. 


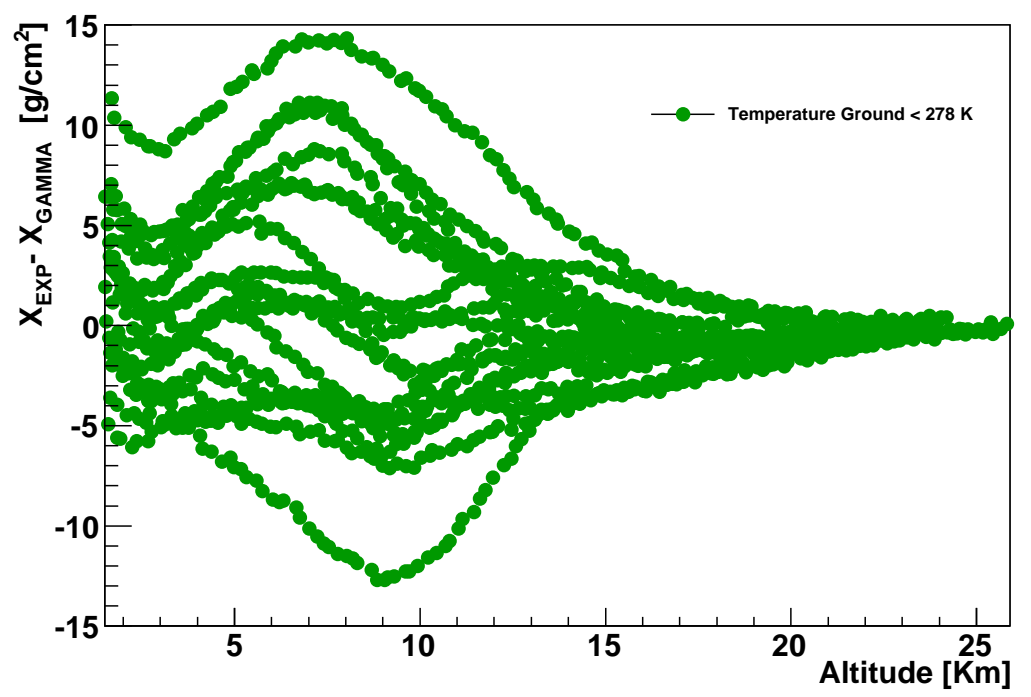

\section{Temperature Ground $<278 \mathrm{~K}$}

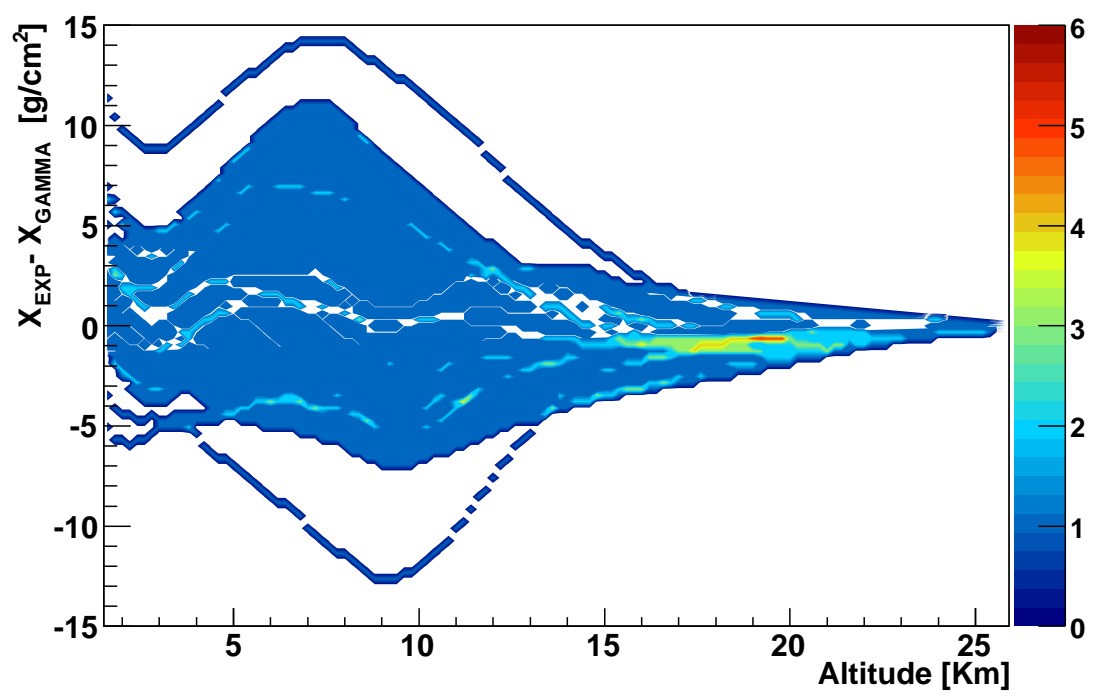

Figura 6.15: Diferencias entre la predicción del modelo GAMMA y el dato experimental para los perfiles con valor de temperatura al nivel del suelo menor a 278 K (Figura estilo scatter-plot arriba y density-plot abajo). Estos perfiles no fueron utilizados en el desarrollo del Modelo GAMMA. 


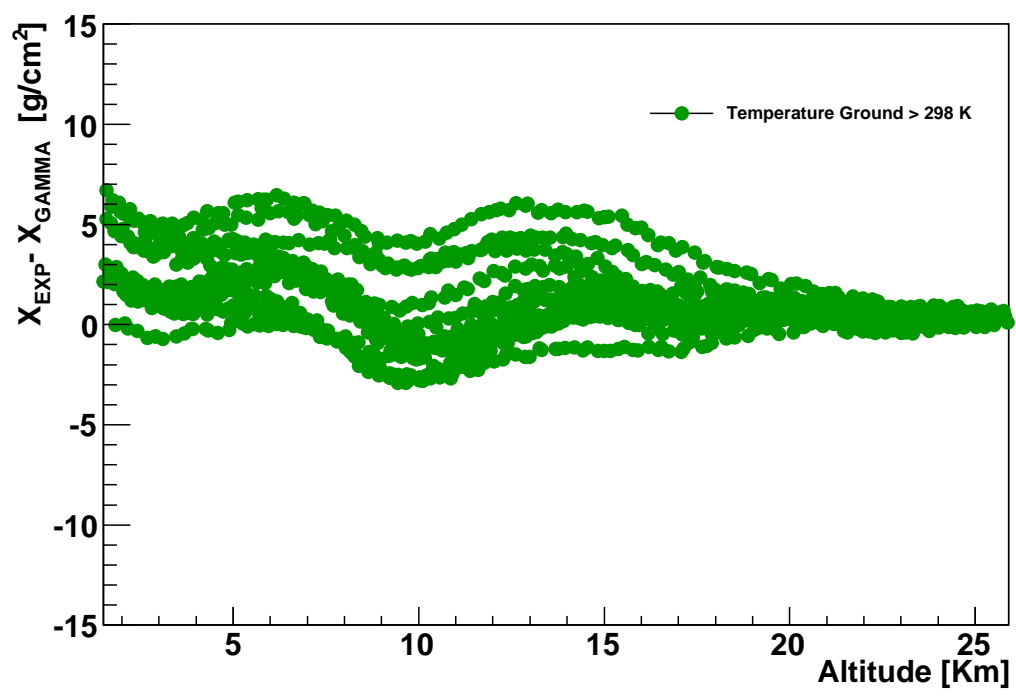

\section{Temperature Ground $>298 \mathrm{~K}$}

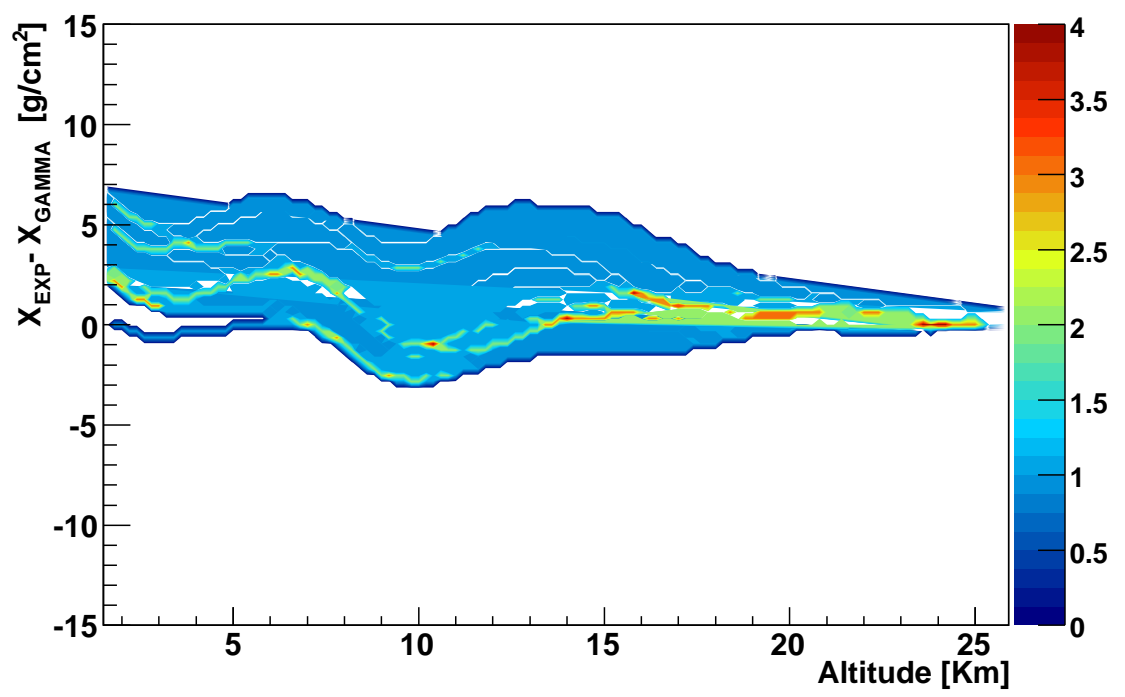

Figura 6.16: Diferencias entre la predicción del modelo GAMMA y el dato experimental para los perfiles con valor de temperatura al nivel del suelo mayor a $298 \mathrm{~K}$ (Figura estilo scatter-plot arriba y density-plot abajo). Estos perfiles no utilizados en el desarrollo del Modelo GAMMA. 


\section{Capítulo 7}

\section{Estudios sobre el Modelo GAMMA}

Este capítulo incluye algunas modificaciones que se hicieron sobre las diferentes posibilidades que se abrían para explorar. Muchas de ellas ya han sido reportadas en los encuentros de la colaboración del Observatorio Pierre Auger [110], [111], [112].

Todos los estudios realizados fortalecen al modelo GAMMA, validando su idea principal del uso de la temperatura al nivel del suelo para caracterizar con un nivel de incerteza mínimo a la profundidad atmosférica al nivel del suelo.

Las diferentes secciones explicitan cada una de las modificaciones y las apreciaciones referidas a su conveniencia en relación a precisión y simpleza.

\subsection{Modelo de capas no isotérmico}

Como la estructura funcional del modelo GAMMA se corresponde con la del modelo de Linsley, y éste representa una modelización de la atmósfera por capas isotérmicas, es claro que en definitiva GAMMA reproduce la misma idea: para cada valor de temperatura en el nivel suelo hay una estructura de capas isotérmicas con una temperatura propia. Sin embargo, como puede apreciarse claramente en la figura 5.2, independientemente de una estructura adecuada de capas, la temperatura dista mucho de ser constante a tramos. Con este tema en consideración, y en base a perfiles de temperaturas colectados en Malargúe, se testeó la posibilidad de usar una nueva función que contemple este punto. Evidentemente esto implicaba utilizar una estructura funcional diferente, cuyo desarrollo se explicita a continuación. 


\subsubsection{Construcción de la función}

Comencemos desde el principio. Esta nueva modelización también incluye modelizar al aire de la atmósfera como el de un gas ideal. Con lo cual la ecuación de estado expresada en términos de la presión $p$, el volumen $V$, el número de moles $n$, la constante universal de los gases $R$ y la temperatura $T$ está dada por

$$
p V=n R T,
$$

y puede reescribirse trivialmente mediante la densidad $\rho$ y la masa molar $M_{m}$ como

$$
\rho=\frac{p M_{m}}{R T}
$$

Por otro lado, del Teorema General de la Fluidostática para un sistema de coordenadas que mide la altura $h$ positiva desde el suelo tenemos

$$
\frac{d p}{d h}=-\rho g
$$

Reemplazando 7.2 en 7.3 , se encuentra

$$
\frac{d p}{d h}=-\frac{p M_{m}}{R T} g
$$

que se puede expresar en la ecuación diferencial

$$
\frac{d p}{p}=-\frac{g M_{m}}{R} \frac{d h}{T}
$$

Suponiendo una dependencia lineal de la temperatura con la altura de la forma

$$
T(h)=T_{0}+\Delta\left(h-h_{0}\right) \quad,
$$

donde $T_{0}$ representa la temperatura al inicio de una capa de la atmósfera cuyo límite inferior está definidio por la altura $h_{0}$, y reemplazando en 7.5 se obtiene

$$
\frac{d p}{p}=-\frac{g M_{m}}{R} \frac{d h}{T_{0}+\Delta\left(h-h_{0}\right)}
$$

Integrando entre $p_{0}$ y $p\left(h_{0} \mathrm{y} h\right)$, se llega fácilmente a

$$
p(h)=p_{0}\left(1+\frac{\Delta\left(h-h_{0}\right)}{T_{0}}\right)^{-\frac{g M_{m}}{R \Delta}}
$$

que representa la variación de la presión con la altura. 
Insertando este último resultado en 7.2 y usando nuevamente 7.6, obtenemos la función densidad, descrita como

$$
\begin{aligned}
\rho(h) & =\frac{M_{m} p_{0}}{R T_{0}}\left(1+\frac{\Delta\left(h-h_{0}\right)}{T_{0}}\right)^{-\frac{g M_{m}}{R \Delta}-1} \\
& =\rho_{0}\left(1+\frac{\Delta\left(h-h_{0}\right)}{T_{0}}\right)^{-\frac{g M_{m}}{R \Delta}-1}
\end{aligned}
$$

Luego considerando la relación entre la densidad y la profundidad atmosférica, y mediante una integración se llega a

$$
\begin{aligned}
X(h) & =\frac{\rho_{0} T_{0} R}{g M_{m}}\left(1+\frac{\Delta\left(h-h_{0}\right)}{T_{0}}\right)^{-\frac{g M_{m}}{R \Delta}} \\
& =X_{0}\left(1+\frac{\Delta\left(h-h_{0}\right)}{T_{0}}\right)^{-\frac{g M_{m}}{R \Delta}}
\end{aligned}
$$

que representa la variación de la profundidad atmosférica con la altura.

Identifiquemos además la función inversa, muy útil en el caso de simulaciones, dada por

$$
h(X)=h_{0}-\frac{T_{0}}{\Delta}\left(1-\frac{\rho_{0} T_{0} R}{g M_{m} X}\right)^{\frac{g M_{m}}{R \Delta}}
$$

Consideremos además que se sigue planteando la estructura de capas, por lo que la expresión 7.10 es en realidad válida en cada capa $l$. Es decir que la función es

$$
X(h)=X_{l}\left(1+\frac{\Delta_{l}\left(h-h_{l}\right)}{T_{l}}\right)^{-\frac{g M_{m}}{R \Delta_{l}}}
$$

donde $T_{l}$ y $\Delta_{l}$ surgen de parametrizar de acuerdo a 7.6 la variación de la temperatura con la altura dentro de la capa $l$.

La función completa que se utlizará en el ajuste será entonces de la forma

$$
X_{G A M M A-N I}(h)=\left\{\begin{array}{cc}
a_{l}\left(1+b_{l}\left(h-h_{l-1}\right)\right)^{-c_{l}} & h_{l-1} \leq h<h_{l} \\
a_{7}-b_{7} \frac{h}{c_{7}} & h_{6} \leq h<h_{7} \\
0 & h \geq h_{7}
\end{array}\right.
$$

donde $l$ toma los valores $l=1, \ldots, 6$. Hemos llamado $h_{l-1}$ al límite inferior de la capa y la función queda definida en términos de los coeficientes $a_{l}, b_{l}$ y $c_{l}$. La función resultante la hemos denominado $X_{G A M M A-N I}(h)$, donde el sufijo NI hace referencia a la característica No Isotérmica distintiva de la modelización. 


\begin{tabular}{|c|c|c|}
\hline Capa & Lím inferior $[\mathrm{km}]$ & Lím superior $[\mathrm{km}]$ \\
\hline $\mathbf{1}$ & 0 & 5 \\
\hline $\mathbf{2}$ & 5 & 11 \\
\hline $\mathbf{3}$ & 11 & 16 \\
\hline $\mathbf{4}$ & 16 & 24 \\
\hline $\mathbf{5}$ & 24 & 40 \\
\hline $\mathbf{6}$ & 40 & 100 \\
\hline $\mathbf{7}$ & 100 & 112.8 \\
\hline
\end{tabular}

Cuadro 7.1: Limites de las capas atmosféricas del modelo GAMMA NI en el ajuste de la base de datos de Malargüe.

\subsection{2. $\quad$ El ajuste}

Con el objeto de comparar con los resultados obtenidos con GAMMA, la función 7.13 se procedió a analizar sobre base de datos descrita en la sección 6.1. Por el mismo motivo los datos fueron agrupados en los mismos bins en valor de temperatura al nivel del suelo descritos anteriormente (ver Cuadro $6.1)$.

Las condiciones que la función de ajuste debería cumplir, también fueron las mismas, a saber:

- Continuidad de la función $X_{G A M M A-N I}(h)$ entre capas $\left(X_{l}(h)=X_{l+1}(h)\right)$.

- Continuidad de la función derivada de $X_{G A M M A-N I}(h)$ entre capas $\left(\rho_{l}(h)=\rho_{l+1}(h)\right)$.

- Como los perfiles disponibles estaban completos hasta menos de $30 \mathrm{~km}$, las funciones $X_{G A M M A-N I}(h)$ y $\rho_{G A M M A-N I}(h)$ debían coincidir con los valores de Linsley para alturas mayores.

Esta elección establecía nuevamente 5 parámetros libres en el ajuste, y por conveniencia en las operaciones fueron elegidos $b_{1}, b_{2}, b_{3}, b_{4}$ y $b_{5}$.

La motivación de las capas no isotérmicas tiene sentido si se consideran límites de capas pertinentes. Para seleccionar los límites de capas que resulten en perfiles más precisos, se observó la variación de la temperatura con la altura en los perfiles colectados en la base datos de Malargüe y que dió origen al modelos GAMMA. En particular, y sólo para el cálculo de los límites de las capas, se agruparon los datos en 6 bines de temperatura entre $274 \mathrm{~K}$ y $298 \mathrm{~K}$ (cada bin cubría un rango de $4 \mathrm{~K}$ ) (Figura 7.1). En base a esos perfiles se decidieron los límites de las capas (Cuadro 7.1) 


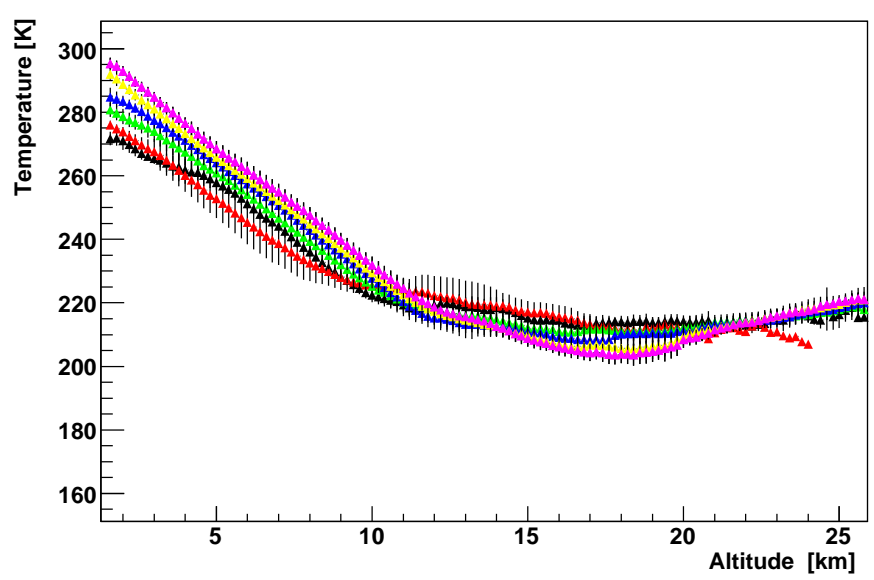

Figura 7.1: Perfiles de temperatura en Malargüe agrupados en 6 bins entre $274 K$ y 298 K de acuerdo al valor de temperatura en el suelo. Cada serie de puntos representa el promedio de perfiles cuyo valor de temperatura al nivel del suelo se encontraba en el mismo rango de $4 \mathrm{~K}$.

\subsubsection{Discusión y Resultados}

Como era de esperar los ajustes resultaron muy precisos. Para contrastar con el modelo GAMMA con capas isotérmicas planteado en el capítulo anterior, se calcularon los perfiles de residuos en cada bin al igual que en las figuras 6.6 y 6.7. Es decir la diferencia entre los datos y el perfil resultante del ajuste del bin. Estos residuos se encuentran graficado en las figuras 7.2 y 7.3 .

Dentro de este estudio se realizó también la comparación considerando todos los perfiles, es decir el equivalente a los perfiles de residuos de las figuras 6.10 .

Es claro que el modelo de capas no isotérmicas reduce las incertezas. En las figuras 7.2 y 7.3 se observa que el valor medio de los residuos es siempre menor a $2 \mathrm{~g} \mathrm{~cm}^{-2}$, además de que se reproduce el mismo efecto que en el caso de capas isotérmicas: las incertezas disminuyen a temperaturas mayores. Todo lleva a suponer que hay una dispersión de datos mayor en los perfiles de baja temperatura.

Al observar la figura 7.4 en comparación con las 6.10, destaca nuevamente la reducción casi al nulo de los valores medios de los residuos. Las incertezas en los perfiles se mantiene en los $4 \mathrm{~g} \mathrm{~cm}^{-2}$.

Un segundo análisis nos llevó a contrastar los ajustes que el modelo de 

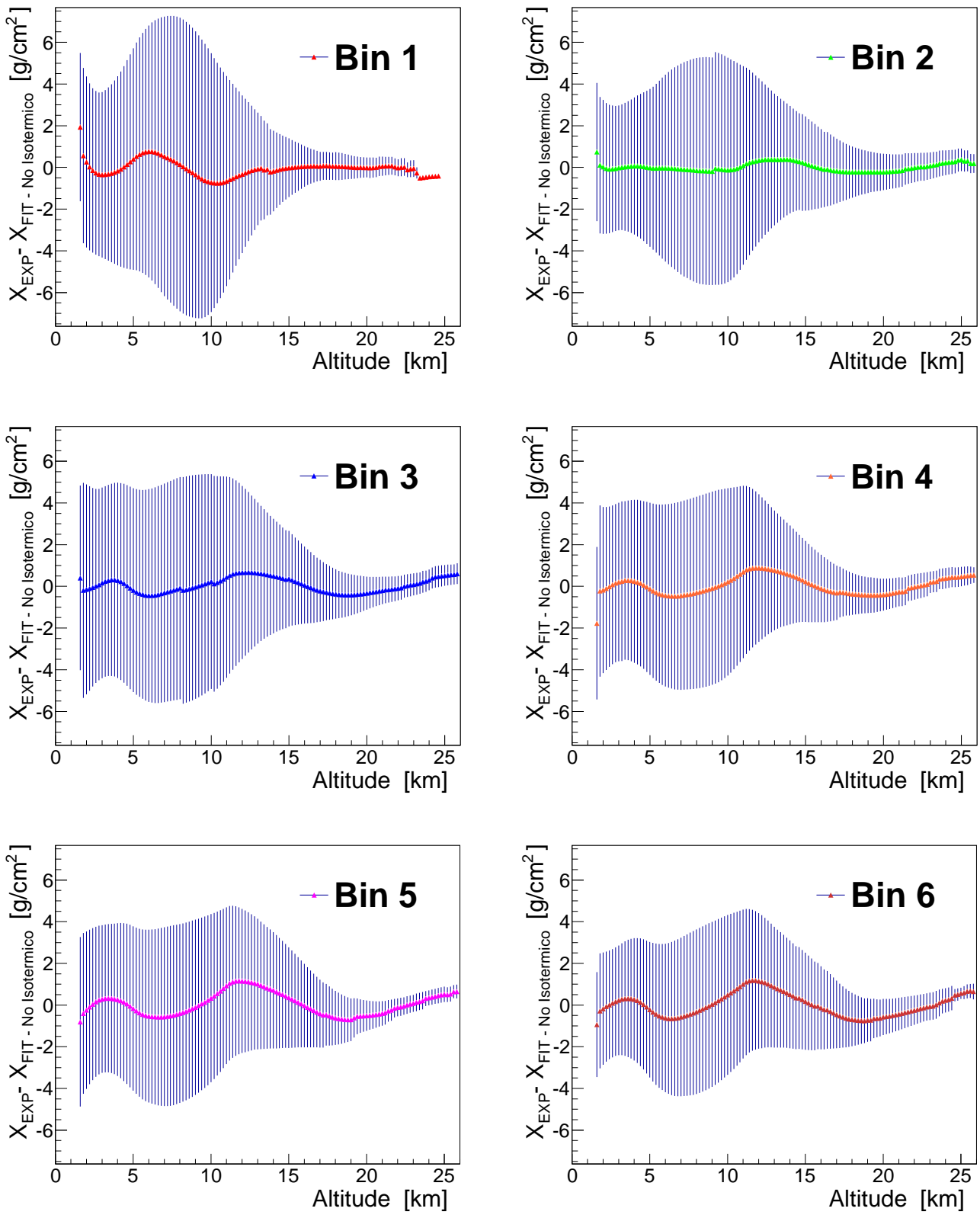

Figura 7.2: Residuos como función de la altura para el Modelo GAMMA con la función No Isotérmica (Bins 1, 2,3, 4, 5 y 6). 

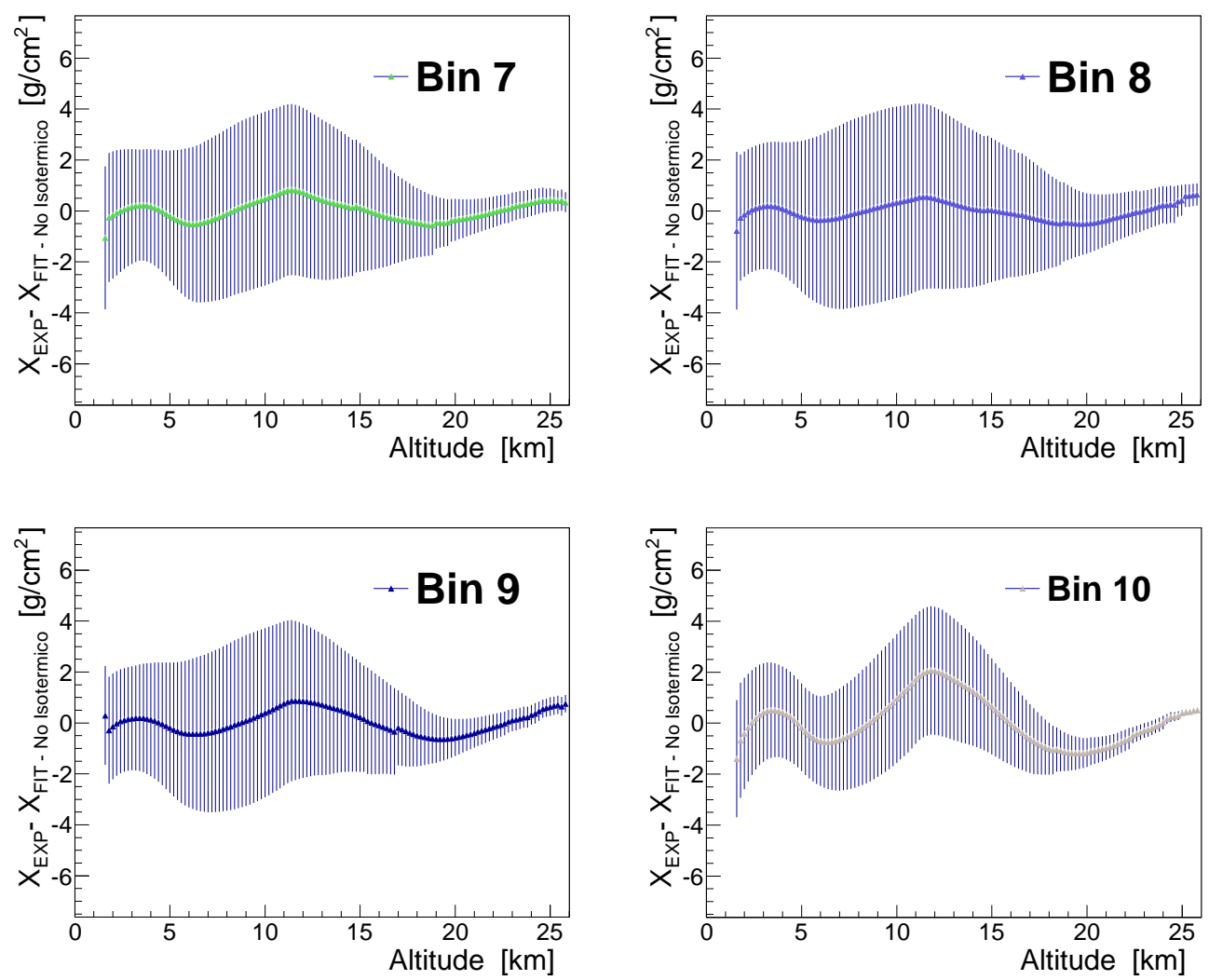

Figura 7.3: Residuos como función de la altura para el Modelo GAMMA con la función No Isotérmica (Bins 7, 8, 9 y 10).

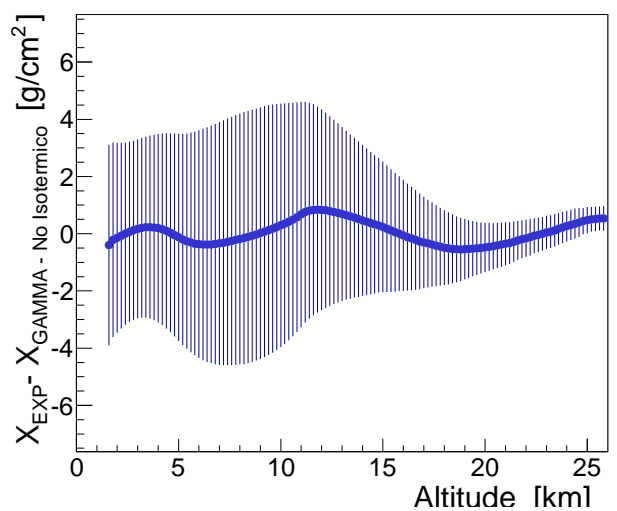

Figura 7.4: Residuos de todos los datos como función de la altura para el Modelo GAMMA con la función No Isotérmica. 
Linsley y GAMMA-NI hacen de la USStdA. Para esta comparación, los límites de las capas fueron elegidos a partir de observar el perfil de temperatura del USStdA (Figura 7.5). Los valores resultantes se encuentran en el Cuadro 7.2

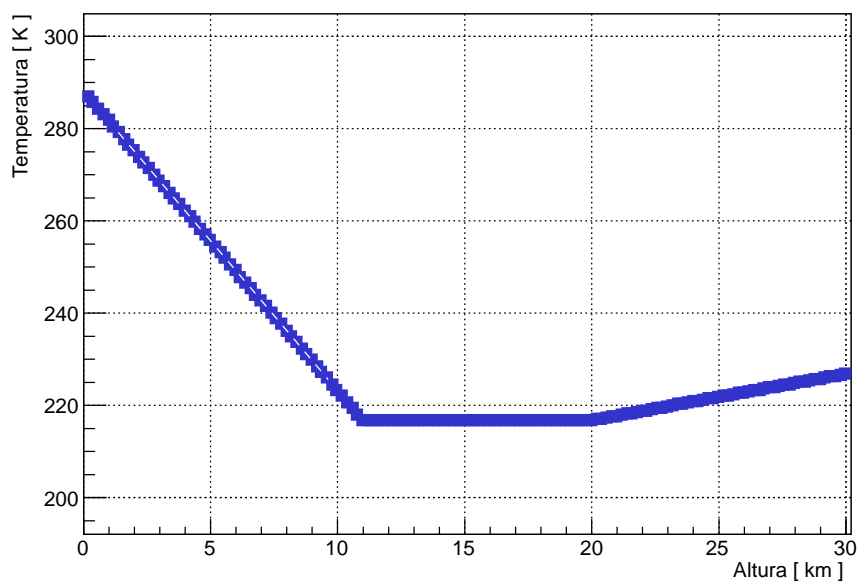

Figura 7.5: Perfil de temperatura de la base de datos USStdA 1976.

\begin{tabular}{|c|c|c|}
\hline Capa & Lím inferior $[\mathrm{km}]$ & Lím superior $[\mathrm{km}]$ \\
\hline $\mathbf{1}$ & 0 & 5 \\
\hline $\mathbf{2}$ & 5 & 11 \\
\hline $\mathbf{3}$ & 11 & 20 \\
\hline $\mathbf{4}$ & 20 & 25 \\
\hline $\mathbf{5}$ & 25 & 40 \\
\hline $\mathbf{6}$ & 40 & 100 \\
\hline $\mathbf{7}$ & 100 & 112.8 \\
\hline
\end{tabular}

Cuadro 7.2: Limites de las capas atmosféricas del modelo GAMMA NI en el ajuste de la USStdA.

Los gráficos que muestran la diferencia en ajustar a la USStdA pueden verse en la Figuras 7.6, donde se ha incluido también el ajuste que realiza de esos datos la función del modelo GAMMA de capas isotérmicas presentada en el capítulo anterior.

Es evidente el buen ajuste que logra GAMMA-NI, con mayor precisión que los otros modelos. Una pequeña intervención vinculada a este tema: tal 

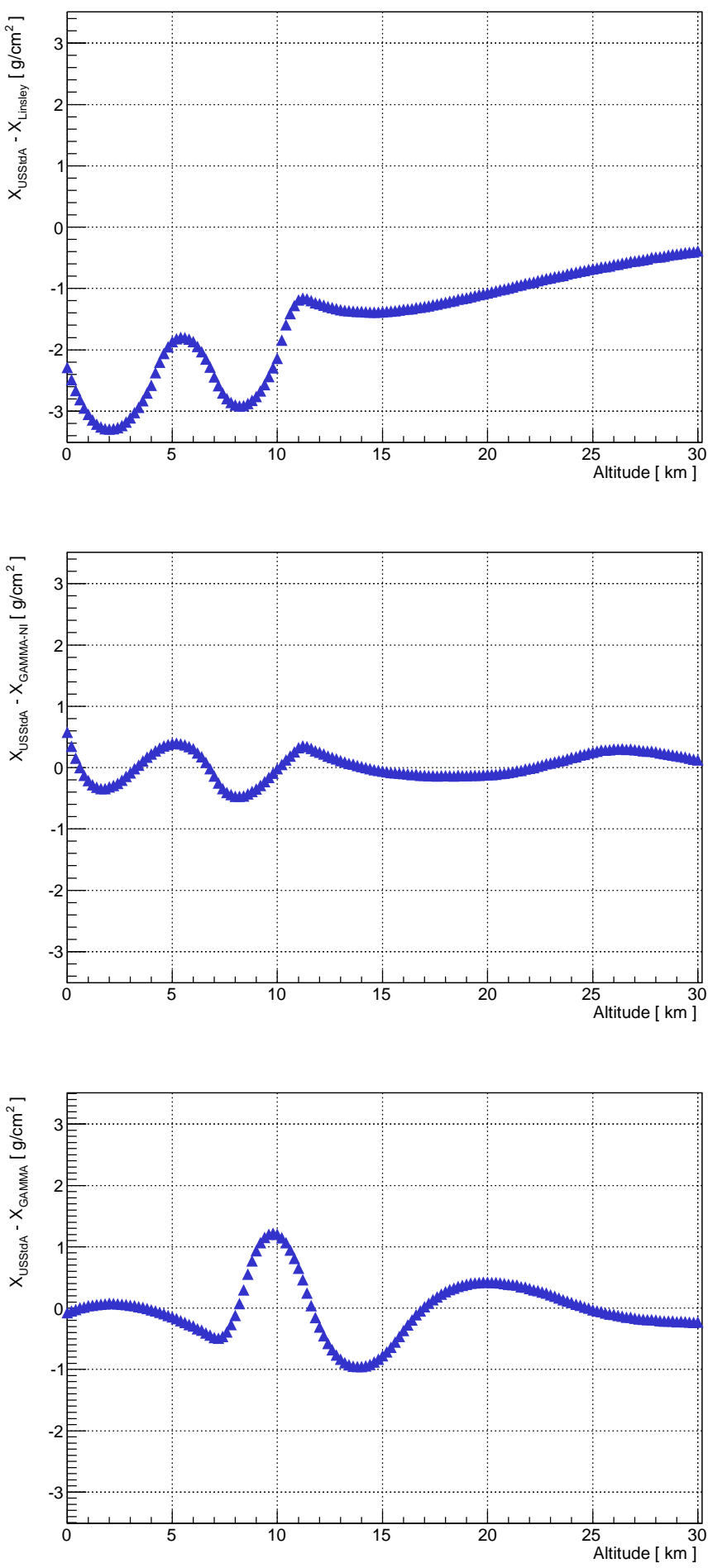

Figura 7.6: Diferencias entre los datos de la USStdA y el modelo de Linsley de 5 capas (arriba), el modelo GAMMA-NI (medio), y el modelo GAMMA (abajo). 
vez resulte muy obvio, pero podría suceder que algún observador se alarme a partir de los resultados de las figuras 7.6 pensando Si el modelo de Linsley surge de los datos de la USStdA, ¿por qué la función GAMMA-NI ajusta mejor esos datos? Hay varios puntos que contribuyen a la respuesta. Primero, no hay que perder de vista el momento histórico: el modelo de Linsley se realizó a comienzos de la década de 1980 y no está claro si los ajustes numéricos fueron realizados con las incipientes computadoras. El modelo GAMMANI, sí fue realizado mediante cálculo numérico por procesadores, con rutinas de minimización de convergencias muy poderosas y precisas. También podemos justificarlo recurriendo al disparador de GAMMA-NI: la posibilidad de suponer capas no isotérmicas a sabiendas de que en la atmósfera (mas allá de una elección adecuada de capas) la variación de la temperatura es notable. Por último la cuantificación de la incerteza: el mayor valor en el perfil de diferencia es aproximadamente de $3.5 \mathrm{~g} \mathrm{~cm}^{-2}$ y ocurre en los primeros metros, cuando el valor de la profundidad atmosférica es de varios cientos de $\mathrm{g} \mathrm{cm}^{-2}$.

Un punto interesante es poder comparar los valores del ajuste con los parámetros físicos involucrados. Efectivamente, de observar las expresiones 7.12 y 7.13 podemos correlacionar los parámetros $a_{l}, b_{l}$ y $c_{l}$ del ajuste con los valores que plantea la base de datos USStdA. Estos valores se reflejan en el Cuadro 7.3 y la correlación entre los mismos demuestra nuevamente la precisión que alcanza esta variante del modelo GAMMA.

\begin{tabular}{|l|c|c|}
\hline Parámetro & USStdA & $\begin{array}{c}\text { GAMMA No } \\
\text { Isotérmico }\end{array}$ \\
\hline \hline $\begin{array}{l}\text { Valor de X al nivel del } \\
\text { suelo }\end{array}$ & $1033.81 \mathrm{~g} \mathrm{~cm}^{-2}$ & $1033.23 \mathrm{~g} \mathrm{~cm}^{-2}$ \\
\hline $\begin{array}{l}\text { Temperatura al nivel } \\
\text { del suelo }\end{array}$ & $288.15 \mathrm{~K}$ & $292.67 \mathrm{~K}$ \\
\hline $\begin{array}{l}\text { Temperatura al inicio } \\
\text { de capa 1 }\end{array}$ & $216.65 \mathrm{~K}$ & $223.31 \mathrm{~K}$ \\
\hline $\begin{array}{l}\text { Temperatura al inicio } \\
\text { de capa 2 }\end{array}$ & $207.65 \mathrm{~K}$ & $209.35 \mathrm{~K}$ \\
\hline
\end{tabular}

Cuadro 7.3: Correlación entre los datos de la USStdA y los parámetros deducibles de los coeficientes obtenidos del ajuste con GAMMA-NI. 


\subsection{Parametrización polinómica de los $c_{l}$}

En el desarrollo del modelo GAMMA, tomamos la decisión de parametrizar los coeficientes $c_{l}(l=1,2,3,4,5)$ como función del valor de la temperatura al nivel del suelo (ver sección 6.3). Esta elección se basaba en mantener una simplicidad para el modelo (el de una relación lineal), pero la variación de los coeficientes con la temperatura dejaba el camino abierto a la posibilidad de perfeccionar el modelo utilizando un ajuste polinómico de los coeficientes de grado mayor a 1 . Con esta idea fue que realizamos un ajuste polinómico de grado 3 sobre los coeficientes $c_{l}$, y que pueden observarse en las siguientes Figuras (incluimos nuevamente las Figuras 6.5 para facilitar la comparación)
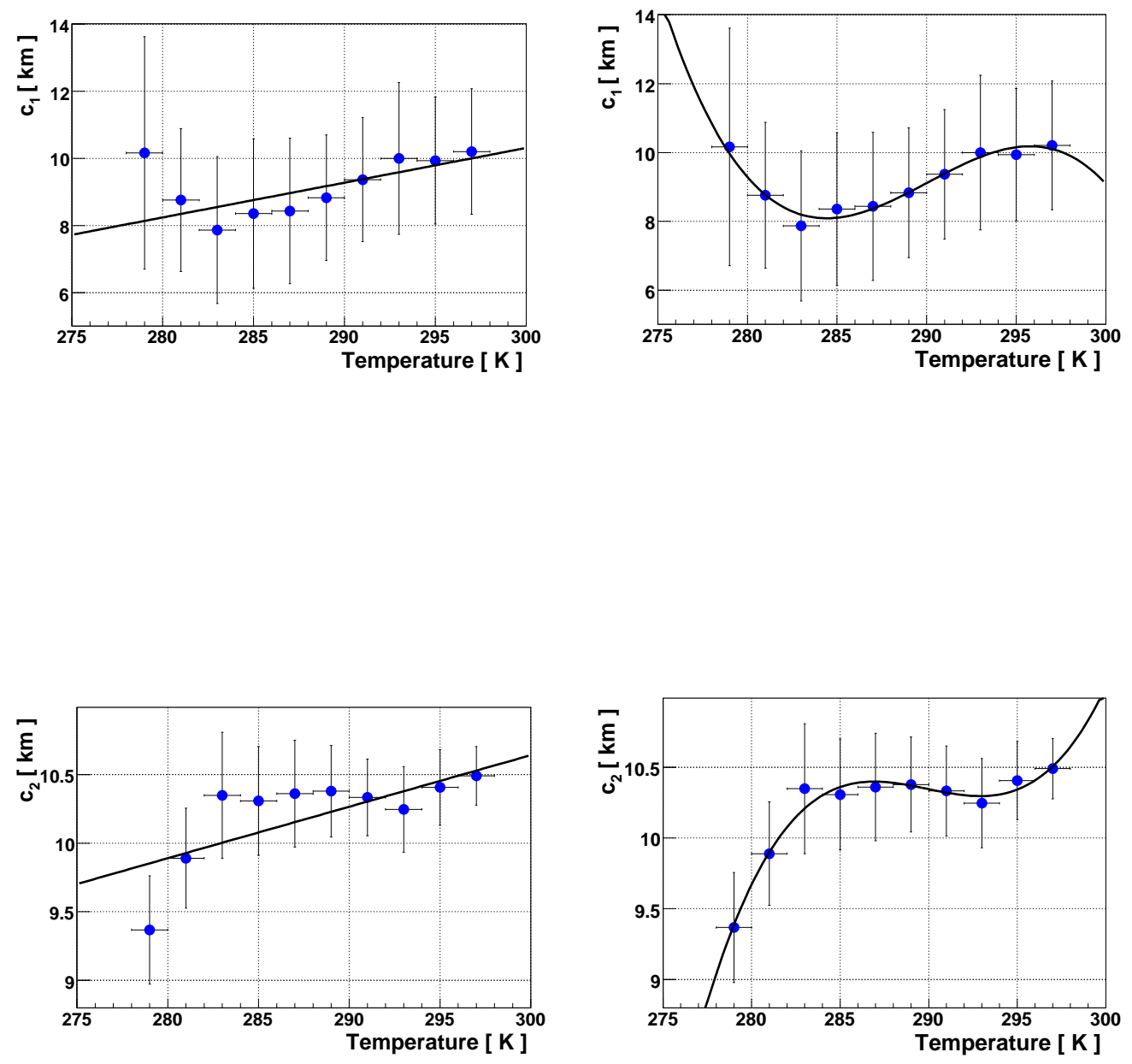

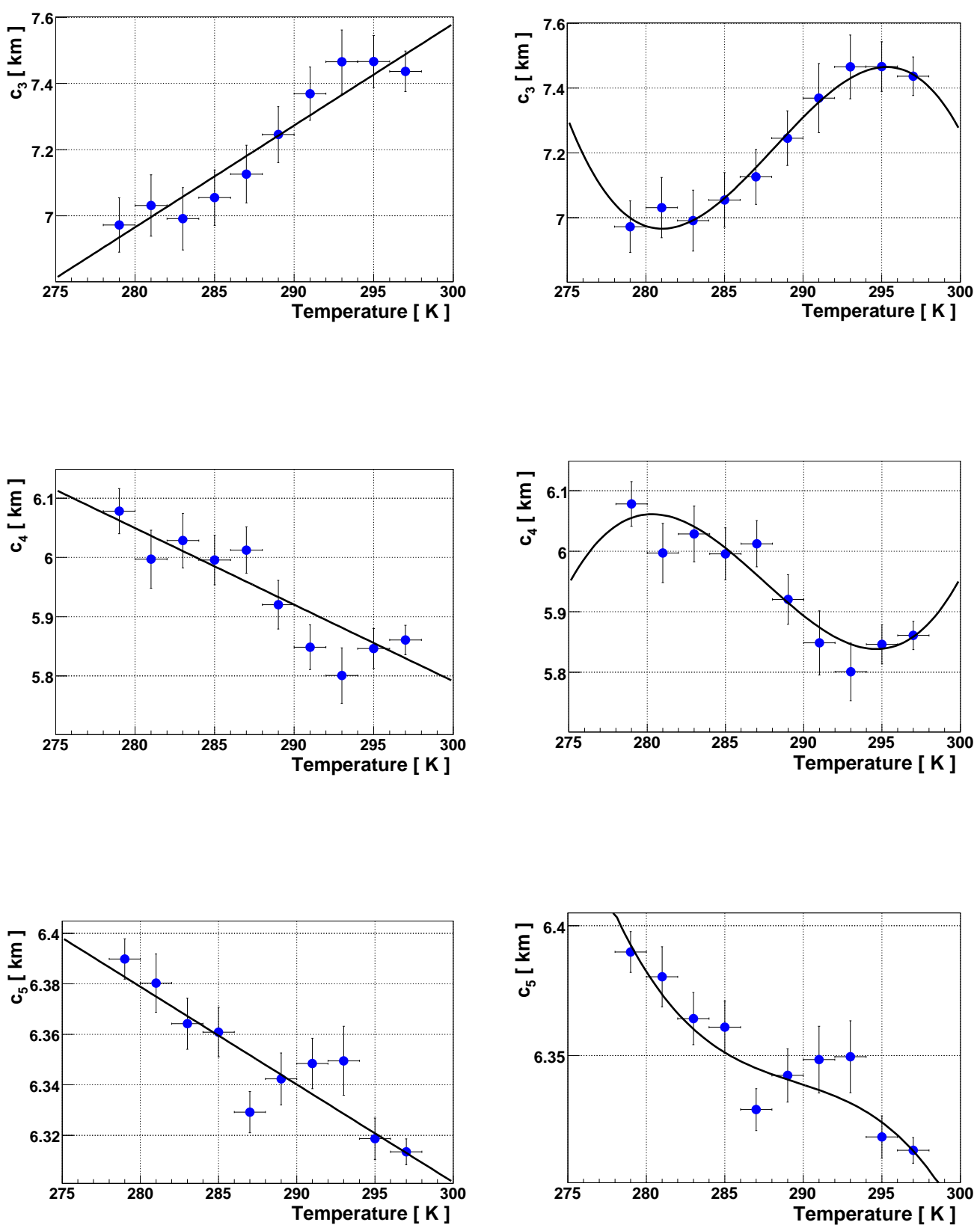

Figura 7.7: Variación de los coeficientes $c_{l}$ del modelo GAMMA con la altura, y un ajuste lineal sobre los mismos (izq) o polinómico de grado 3 (der).

Con estos nuevos ajustes, se procedieron a calcular los residuos. En estos gráficos, los residuos fueron calculados teniendo en cuenta la temperatura media del bin $\left(T_{G R O U N D-B I N}\right)$. Es decir, con el valor de $T_{G R O U N D-B I N}$ se 
calcularon los valores de $c_{l}$ lo que permitieron calcular las funciones medias predichas por el modelo GAMMA para ese bin $X_{G A M M A-B I N}(h)$. Por último se calcularon los residuos para cada altura, haciendo

$$
\operatorname{Residuo}_{G A M M A-B I N}(h)=X_{E X P}(h)-X_{G A M M A-B I N}(h)
$$

donde $X_{E X P}(h)$ es el valor experimental para cada perfil para cada altura $h$. En la columna izquierda de la siguiente figura, pueden observarse los residuos obtenidos en cada bin utilizando el ajuste lineal en los $c_{l}$ (tal como fueron desarrollados en el Modelo GAMMA), mientras que en la columna derecha se encuentran los residuos calculados con el ajuste polinómico de grado 3.

A primera vista es destacable que, como en ambos casos se calcularon las funciones $X_{\text {GAMMA-BIN }}(h)$, por esta razón los residuos del ajuste lineal no son los mismos de las Figuras 6.6 y 6.7, ya que estos últimos se calculaban con la temperatura al nivel del suelo de cada perfil.

La observación más precisa de estos gráficos resultantes (Figuras 7.8, 7.9, 7.10 y 7.11) conduce a interpretar una mejora en el uso de los polinomios de grado 3. La diferencia mayor se observa en el bin 1, aunque dentro de la incerteza no llega a ser excesiva. De todas maneras, en general son muy similares las predicciones (fundamentalmente en los Bins 2 a 10) aunque no logran disminuir la dispersión en las incertezas. Observamos también que la estructura funcional de los residuos se mantiene, conduciendo a pensar en trabajar a futuro en la estructura de capas para minimizar las diferencias.

\subsection{Modelo aumentando grados de libertad}

Como fue planetado en el capítulo anterior, para el modelo GAMMA se utilizó la dependencia de los coeficientes $c_{l}$ con la Temperatura en el nivel del suelo $T_{G R O U N D}$ en la forma lineal

$$
c_{l}\left(T_{\text {GROUND }}\right)=M_{l} T_{\text {GROUND }}+N_{l} \quad(l=1, \ldots, 5)
$$

para determinar la dependencia con el modelo con éste último parámetro. Esta función se obtuvo a partir de 10 valores correspondientes a los 10 bines en Temperatura en el suelo obtenidos de la base de datos y los correspondientes cortes. Frente a este formato para los parámetros $c_{l}$ y su relación con la función del perfil $X(h)$, se decidió optar por incluir la expresión 7.3 directamente en la función de ajuste. Con esta modificación, la nueva función de 

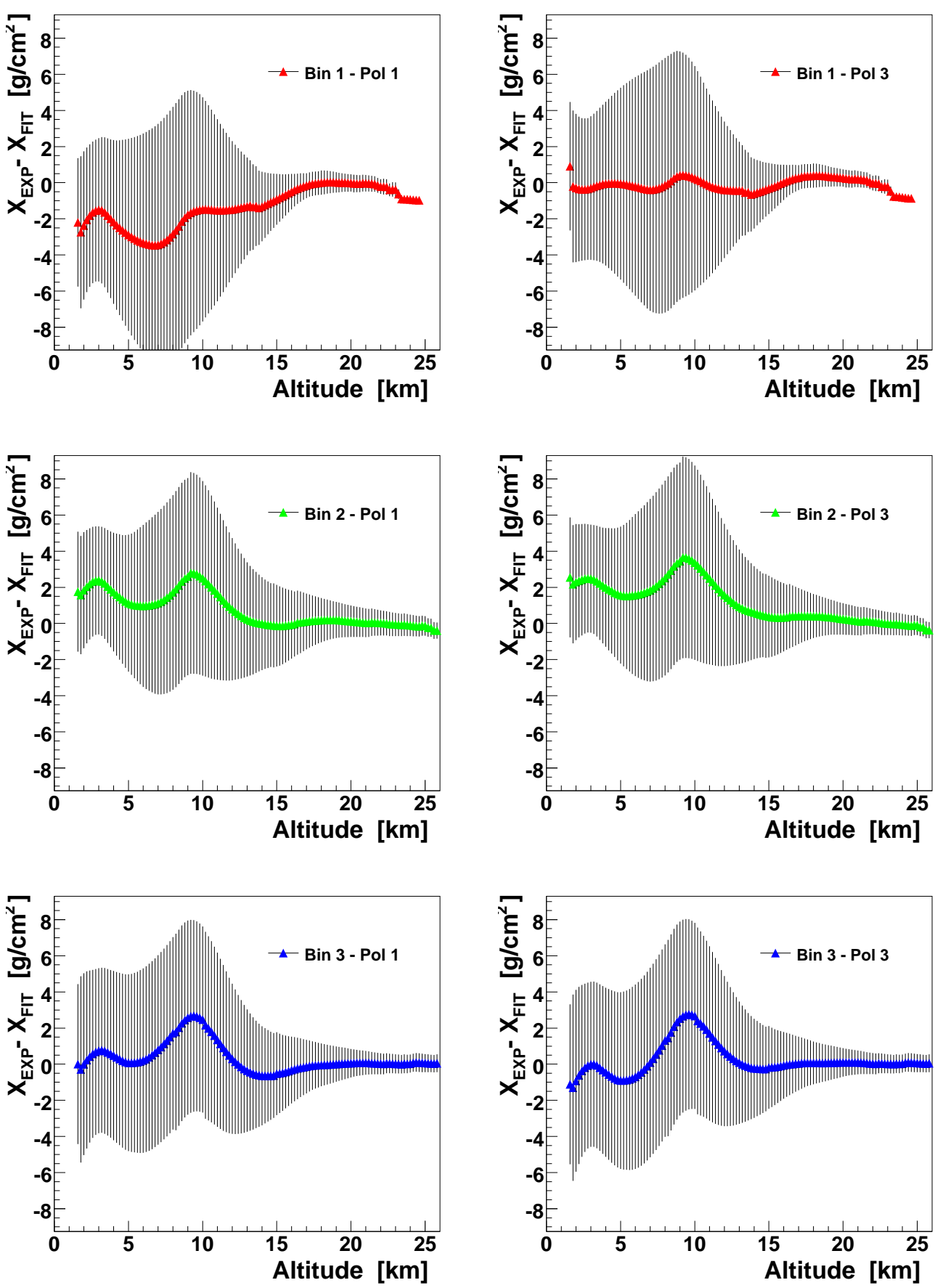

Figura 7.8: Comparación entre Residuos como función de la altura para el Modelo GAMMA, obtenidos mediante una parametrización lineal de los coeficientes $c_{l}$ (izq.) o polinómica de grado 3 (der.) (Bins 1, 2 y 3). 

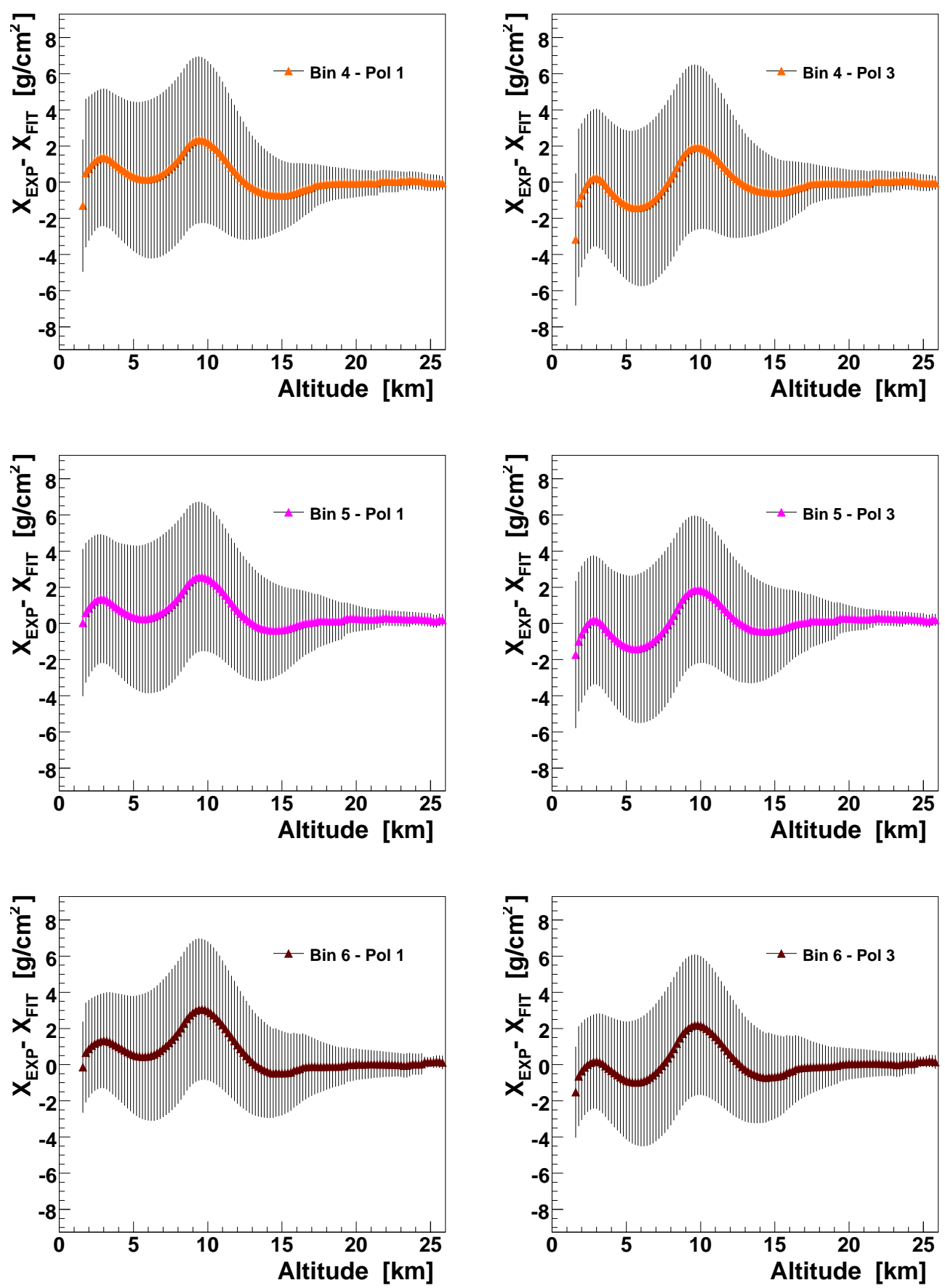

Figura 7.9: Comparación entre Residuos como función de la altura para el Modelo GAMMA, obtenidos mediante una parametrización lineal de los coeficientes $c_{l}$ (izq.) o polinómica de grado 3 (der.) (Bins 4, 5 y 6). 

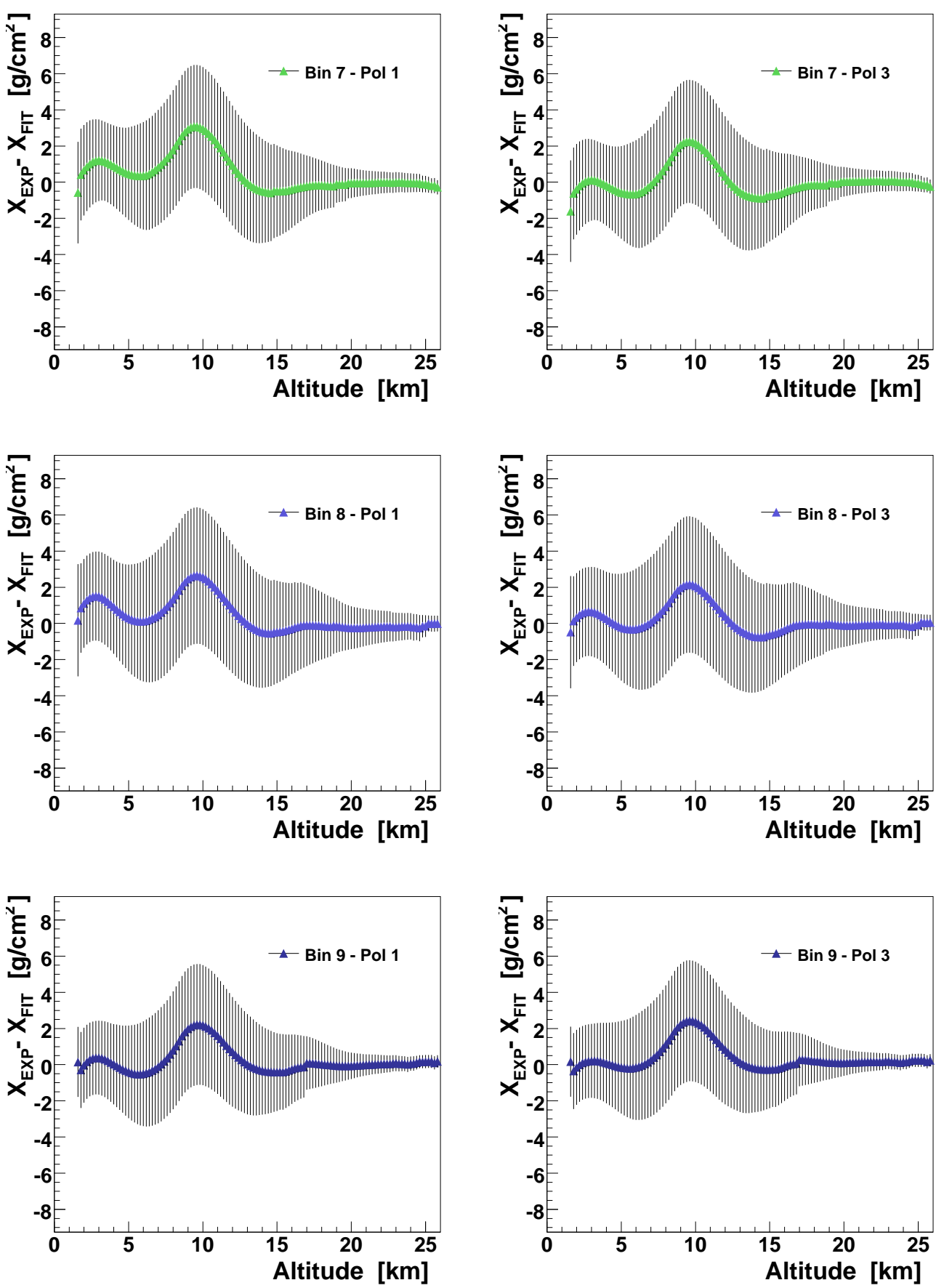

Figura 7.10: Comparación entre Residuos como función de la altura para el Modelo GAMMA, obtenidos mediante una parametrización lineal de los coeficientes $c_{l}$ (izq.) o polinómica de grado 3 (der.) (Bins 7, 8 y 9). 

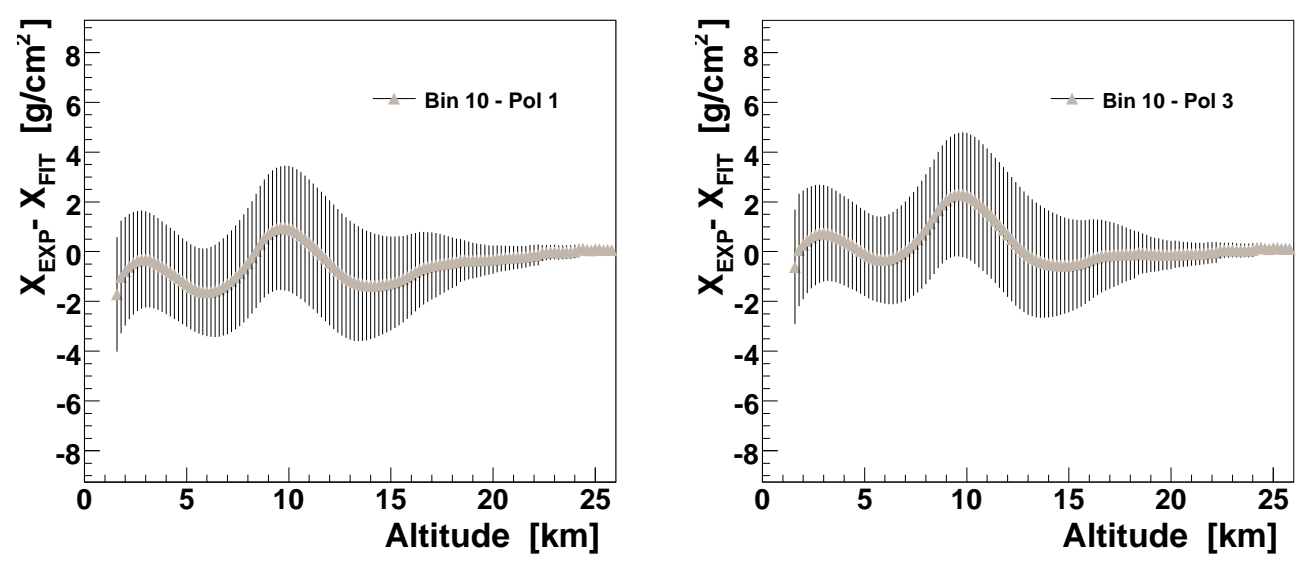

Figura 7.11: Comparación entre Residuos como función de la altura para el Modelo GAMMA, obtenidos mediante una parametrización lineal de los coeficientes $c_{l}$ (izq.) o polinómica de grado 3 (der.) (Bin 10).

ajuste que llamaremos GAMMA II queda

$$
X_{\text {GAMMAII }}=\left\{\begin{array}{cc}
a_{l}+b_{l} e^{-\frac{h}{M_{l} T_{G R O U N D}+N_{l}}} & h_{l-1} \leq h<h_{l} \\
a_{7}-b_{7} \frac{h}{c_{7}} & h_{6} \leq h<h_{\text {lim }} \\
0 & h \geq h_{\text {lim }}
\end{array}\right.
$$

donde la cantidad de capas está dada nuevamente por $l=1, \ldots, 6$. Asimismo, en lugar de realizar el ajuste a cada set de datos correspondientes al mismo globo, se procedió a realizar el ajuste sobre toda la base de datos simultáneamente, lo que significa encontrar los valores de $M_{l}$ y $N_{l}(l=1, \ldots, 5)$ que hacen mínimo $S$ en la expresión

$$
S=\sum_{m=1}^{M}\left(X_{m}-X\left(h_{m}, T g_{m} \mid M_{1}, M_{2}, M_{3}, M_{4}, M_{5}, N_{1}, N_{2}, N_{3}, N_{4}, N_{5}\right)\right)^{2}
$$

donde $M$ es la cantidad de datos disponibles, y $X_{m}$ el m-ésimo dato de un globo correspondiente a la altura $h_{m}$ y temperatura en el suelo $T g_{m}$.

En definitiva encontrar el valor mínimo de $S$ significaba ajustar la serie completa de datos para cada valor de altura y temperatura en el suelo (Figura 7.12). Para realizar esta minimización se utilizó el algoritmo MINUIT incluido como paquete en el software ROOT [56],

Se graficó luego la diferencia entre el valor experimental y la predicción de GAMMA II para la correspondiente altura y temperatura en el suelo (Figura 


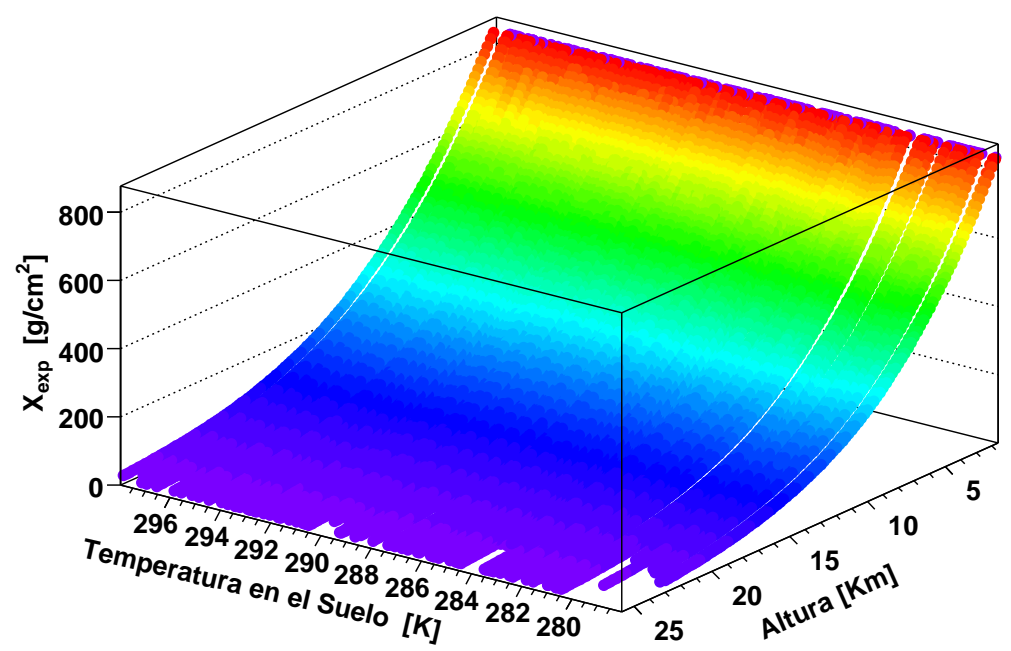

Figura 7.12: Presentación del conjunto de datos a ajustar $X\left(h_{m}, T g_{m}\right)$.

7.13). Debido a la visible semejanza con la predicción de GAMMA vista en el capítulo anterior (Figura 6.8), es que se quiso observar las diferencias entre las predicciones de ambos modelos para los mismos puntos (Figura 7.14). Para toda altura la diferencia es menor a $1 \mathrm{~g} \mathrm{~cm}^{2}$ lo que creemos valida la simpleza y ajustada precisión del modelo original.

\subsection{Estudio en otros sitios}

Con el objeto de estudiar la posibilidad de extender el Modelo GAMMA, desarrollando un modelo de alcance global, se procedió al inicio de un estudio acerca de reproducir el desarrollo de GAMMA en otras latitudes. Este es un estudio que sirve como punto de partida para uno de los alcances más ambiciosos en el desarrollo del modelo y explora si el uso de la temperatura al nivel del suelo continúa siendo un buen parámetro para la caracterización de perfiles.

Los sitios elegidos para comenzar el estudios fueron Dodge City (Kansas E.E.U.U.) y la Base Halley en la porción de antártida británica. La elección de estos sitios estuvo ligada a que ambos lugares son cercanos a experimentos de rayos cósmicos, Dodge City se encuentra a menos de $50 \mathrm{~km}$ de Lamar (Colorado) donde se instalaría el sitio Norte del Observatorio Pierre Auger ${ }^{1}$

\footnotetext{
${ }^{1}$ La construcción del sitio Norte del Observatorio Auger en la localidad de Lamar nunca
} 


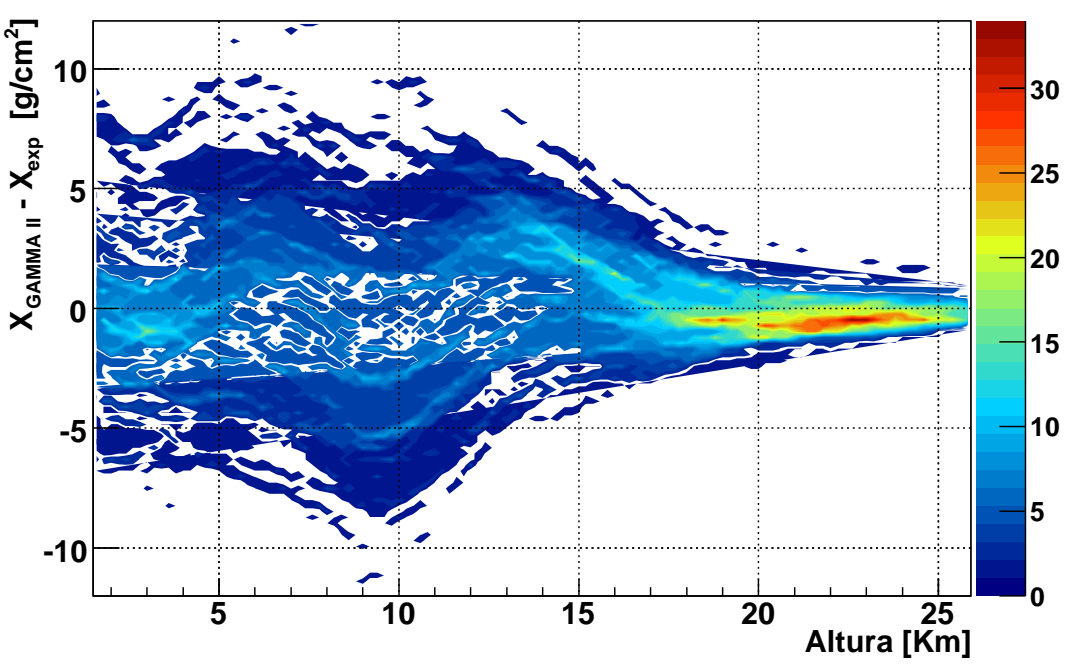

Figura 7.13: Diferencias en la profundidad atmosférica entre cada dato y su predicción de acuerdo a la función GAMMA II.

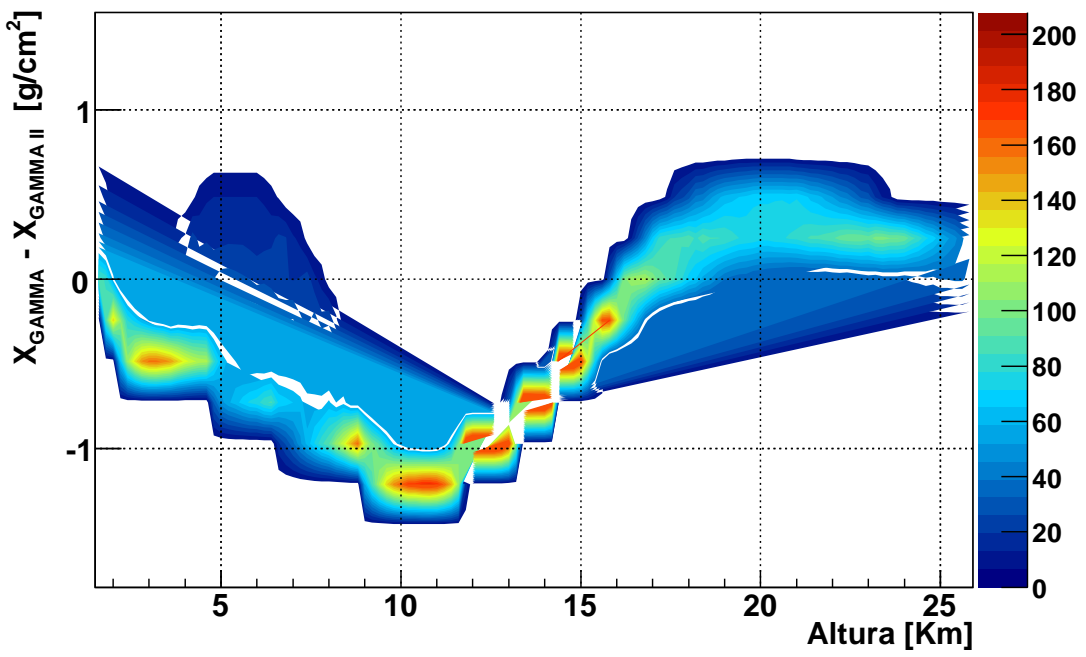

Figura 7.14: Diferencias entre las predicciones de profundidad atmosférica para la función GAMMA y la función GAMMA II. 
y Halley está cerca del conocido experimento IceCube.

Los datos fueron obtenidos, con el permiso correspondiente, del sitio de la Universidad de Wyoming [113], donde se registran las mediciones de una gran variedad de sitios donde se producen periódicos lanzamientos de globos aerostáticos.

A continuación una síntesis del procedimiento realizado, y luego una mínima descripción de los sitios con los resultados obtenidos de la aplicación de modelo GAMMA.

\subsubsection{Síntesis del Procedimiento}

La forma de trabajar con estos datos fue similar a la descrita en el capítulo anterior. Incluyó una primera descarga de las medidas de los sitios con la correspondiente depuración de encabezados y parámetros innecesarios. De cada globo se consideraban las medidas de altura, presión, temperatura y humedad.

Utilizando las expresiones de la sección 6.1 se calcularon los perfiles correspondientes. La secuencia es la siguiente: mediante la ecuación 6.2 se calcula $M_{m}$ que es utilizada junto con las medidas de Temperatura y presión en la ecuación 6.1 para determinar los perfiles de densidad $\rho(h)$. Por último los perfiles de profundidad atmosférica $X(h)$ se calculan haciendo uso de la ecuación 6.7 conjuntamente con 6.8 y 6.9. Es destacable que en este caso el valor de la temperatura en el suelo es un dato incluido en cada perfil medido.

A diferencia de la base de datos de Malargüe, los datos no fueron extrapolados, por lo cual los puntos de los perfiles no estaban equiespaciados cada $200 \mathrm{~m}$. Esto será realizado en los futuros armados de bases de datos.

En forma condensada, el resto del procedimiento es el descrito en la sección 6.1 ; se dividieron los perfiles acorde el valor de la temperatura en el suelo y se ajustaron con la función propuesta 6.12 considerando los mismos límites para las capas planteados en el Cuadro 6.2.

\subsubsection{Dodge City}

Dodge City (DDC) (paradójicamente capital del condado de Ford) se encuentra a $240 \mathrm{~km}$ al este de Lamar, concretamente a $37^{\circ} 45^{\prime} \mathrm{N} 100^{\circ} 1^{\prime} \mathrm{O}$ y con una elevación de $790 \mathrm{~m}$ sobre el nivel del mar. Corresponde a un clima desértico, que incluye un historial de tornados, donde la temperatura media durante los meses de Noviembre a Febrero que varía de $-1^{\circ} \mathrm{C}$ a $5{ }^{\circ} \mathrm{C}$ y en Julio-Agosto de $26^{\circ} \mathrm{C}$.

superó el status de proyecto y actualmente se encuentra suspendido. 
Como fue planteado en la subsección anterior, y reiterando el desarrollo original de GAMMA, los perfiles fueron agrupados en bines de acuerdo al valor de la temperatura en el suelo. El período estudiado comprende los lanzamientos de globos producidos entre enero de 2006 y abril 2007, mayormente realizados a las 0 hs y a las 12 hs. Los agrupamientos resultantes se muestran en el Cuadro 7.4.

\begin{tabular}{|c|c|c|}
\hline Bin & T range $[\mathbf{K}]$ & Balloons \\
\hline \hline $\mathbf{1}$ & $278 \mathrm{~K}-280 \mathrm{~K}$ & 32 \\
$\mathbf{2}$ & $280 \mathrm{~K}-282 \mathrm{~K}$ & 29 \\
$\mathbf{3}$ & $282 \mathrm{~K}-284 \mathrm{~K}$ & 27 \\
$\mathbf{4}$ & $284 \mathrm{~K}-286 \mathrm{~K}$ & 48 \\
$\mathbf{5}$ & $286 \mathrm{~K}-288 \mathrm{~K}$ & 26 \\
$\mathbf{6}$ & $288 \mathrm{~K}-290 \mathrm{~K}$ & 38 \\
$\mathbf{7}$ & $290 \mathrm{~K}-292 \mathrm{~K}$ & 38 \\
$\mathbf{8}$ & $292 \mathrm{~K}-294 \mathrm{~K}$ & 41 \\
$\mathbf{9}$ & $294 \mathrm{~K}-296 \mathrm{~K}$ & 30 \\
$\mathbf{1 0}$ & $296 \mathrm{~K}-298 \mathrm{~K}$ & 28 \\
\hline
\end{tabular}

Cuadro 7.4: Bines de Temperatura utilizados en Dodge City

El ajuste de la función propuesta fue muy preciso. Este punto puede ser observado en los perfiles de los residuos, definidos mediante

$$
\begin{array}{r}
\operatorname{Residuo}_{D D C} \operatorname{GAMMA}_{A}(h)=X_{E X P}(h)-X_{D D C G A M M A}(h) \\
\operatorname{Residuo}_{D D C \text { Linsley }}(h)=X_{E X P}(h)-X_{\text {Linsley }}(h)
\end{array}
$$

El primero de los perfiles de residuos es obtenido a partir del valor experimental $X_{E X P}(h)$ y la función $X_{D D C G A M M A}(h)$ reconstruida usando la temperatura media del bin y está representado en la columna izquierda de las Figuras 7.15, 7.16, 7.17 y 7.18. El perfil de residuos 7.19 es obtenido usando el dato experimental la función de Linsley y se muestra en la columna de la derecha de las mismas figuras.

De manera similar a lo realizado en el desarrollo de GAMMA, se graficaron los coeficientes $c_{l}(\mathrm{i}=1,2,3,4,5)$ obtenidos en el ajuste en cada bin, con el objeto de observar la variación con el valor de la temperatura al nivel del suelo. El resultado se observa en la Figura 7.19.

Remarquemos brevemente algunos puntos llamativos surgidos de lo realizado en este estudio. 

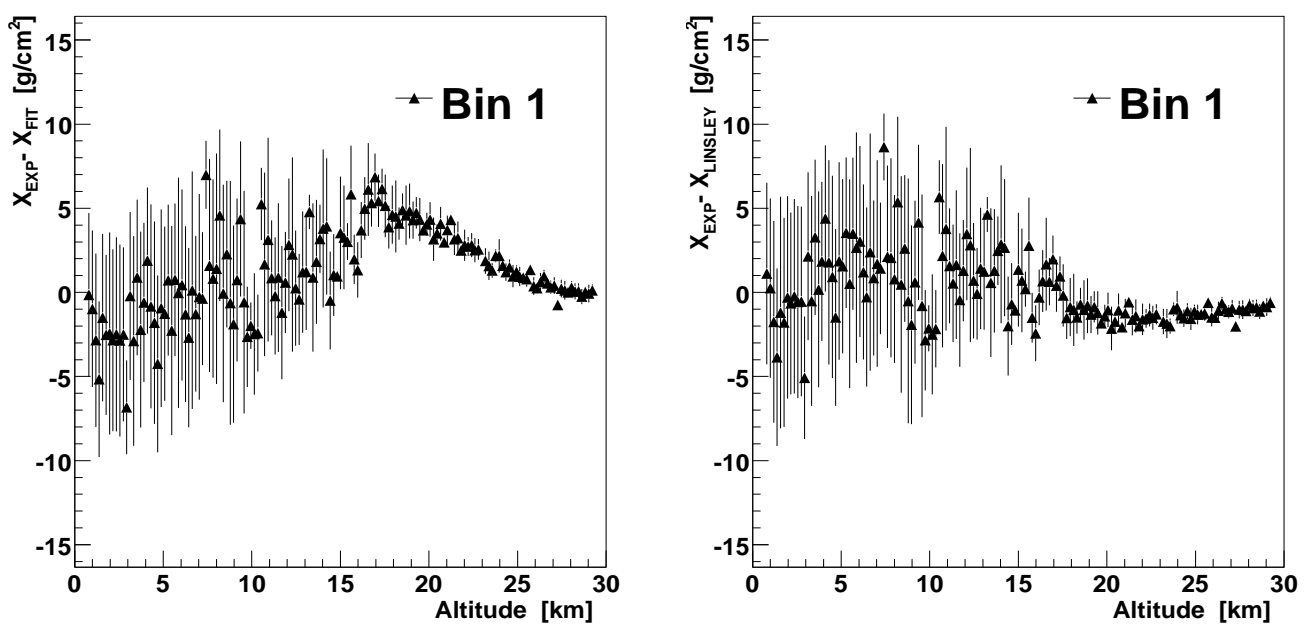

Figura 7.15: Desarrollo del Modelo GAMMA en Dodge City. Residuos considerando el ajuste de GAMMA (izq) y la función de Linsley (Bin 1).

- El modelo de Linsley, desarrollado usando los datos de la USStandard Atmosphere, coincide razonablemente con la serie de datos experimentales (Figuras 7.15 a 7.18). Esto es natural, ya que los datos con los que se construyó la USStandard Atmosphere incluyen a los del centro de los Estados Unidos. Sin embargo, se observa en este primer estudio un pequeño incremento (en la diferencia) a mayores temperaturas al nivel del suelo.

- Los residuos del ajuste GAMMA son similares en todos los bins pero no tienen la estructura "oscilante" que se percibía en la base de datos de Malargüe. Esto podría deberse a que estos datos no tienen el tratamiento de extrapolación en $200 \mathrm{~m}$ que se realizó con los datos del Observatorio Auger. Por otro lado, considerando los valores de la profundidad atmosférica en los estudios de rayos cósmicos, se estima que por encima de los $10 \mathrm{~km}$ los residuos son proporcionalmente demasiado altos.

- Como era esperable en la búsqueda de un modelo global, los coeficientes $c_{i}$ en Dodge City son del mismo orden que los obtenidos con GAMMA en Malargüe. Sin embargo hay leves diferencias y no se observa en ellos un comportamiento lineal con la temperatura. 

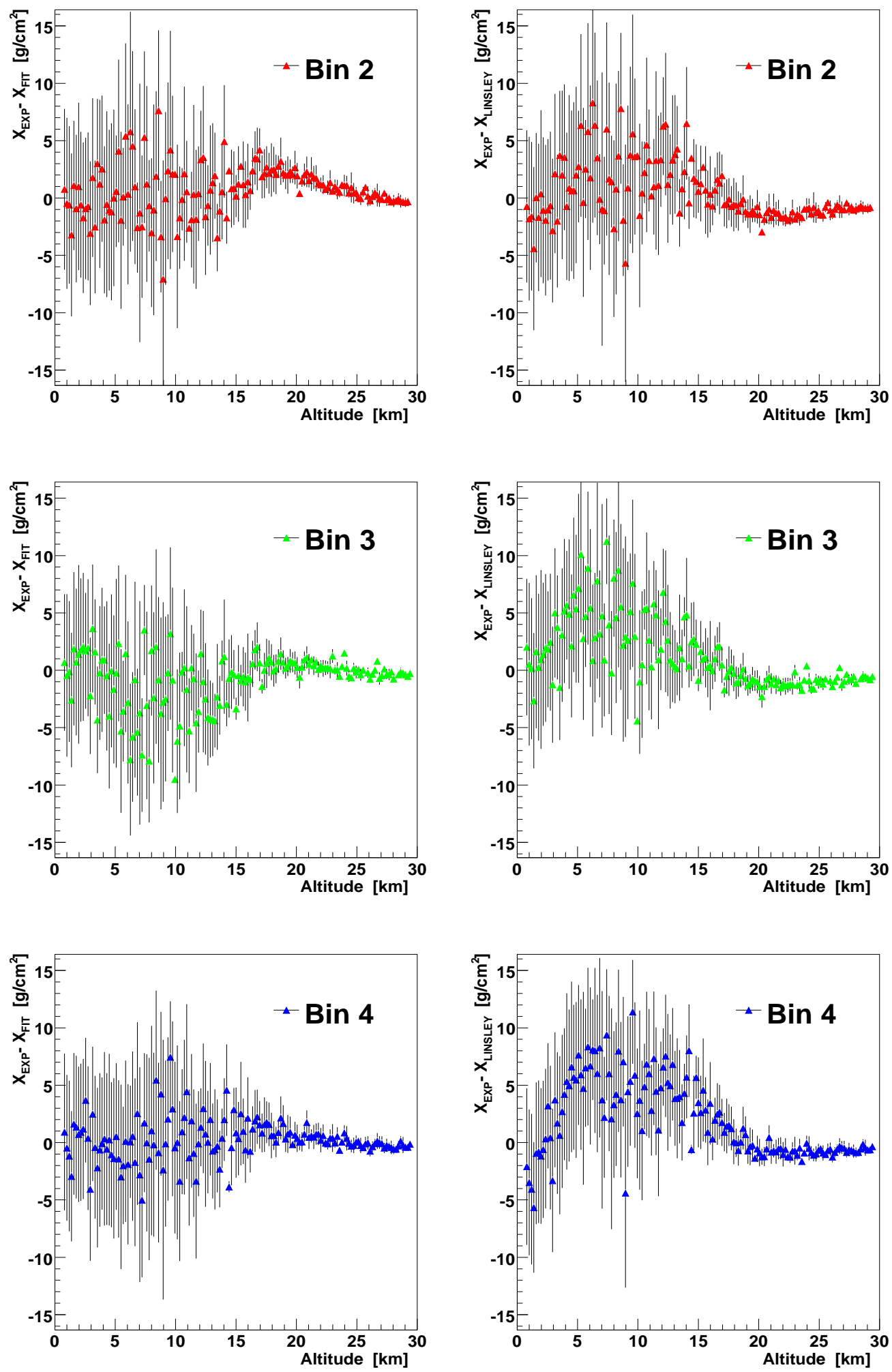

Figura 7.16: Desarrollo del Modelo GAMMA en Dodge City. Residuos considerando el ajuste de GAMMA (izq) y la función de Linsley (Bins 2, 3 y 4). 

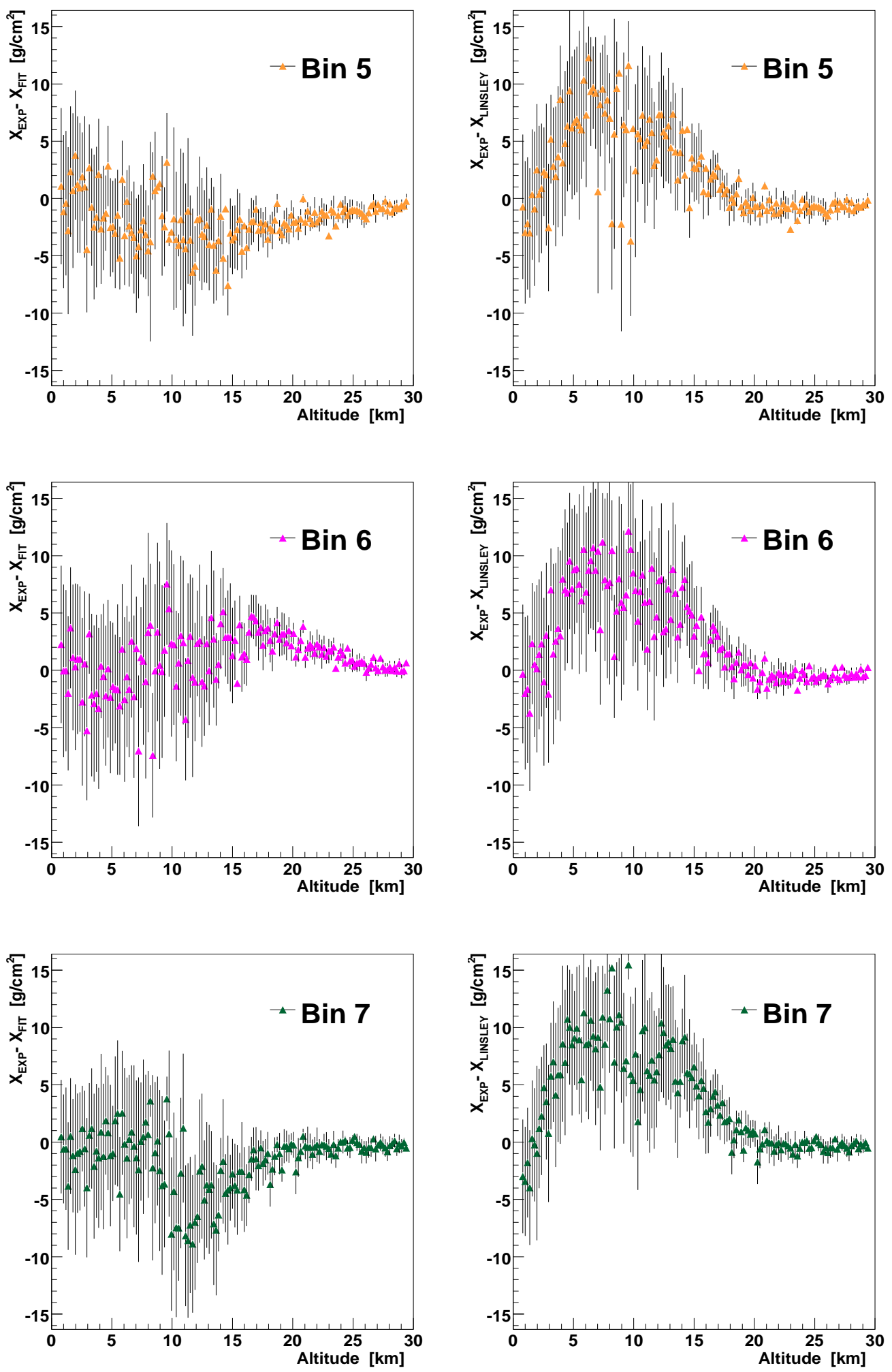

Figura 7.17: Desarrollo del Modelo GAMMA en Dodge City. Residuos considerando el ajuste de GAMMA (izq) y la función de Linsley (Bins 5, 6 y 7). 

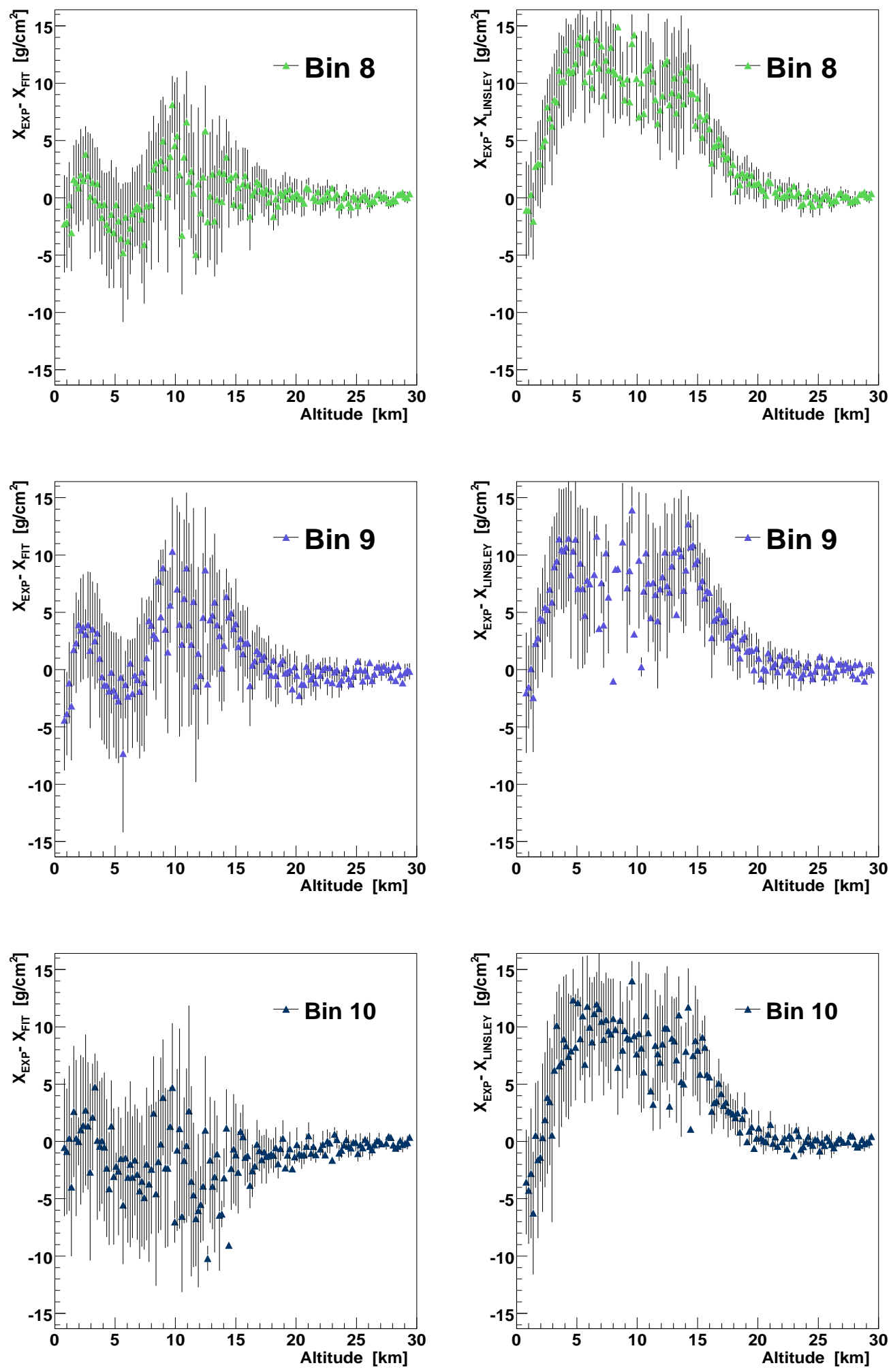

Figura 7.18: Desarrollo del Modelo GAMMA en Dodge City. Residuos considerando el ajuste de GAMMA (izq) y la función de Linsley (Bins 8, 9 y 10). 

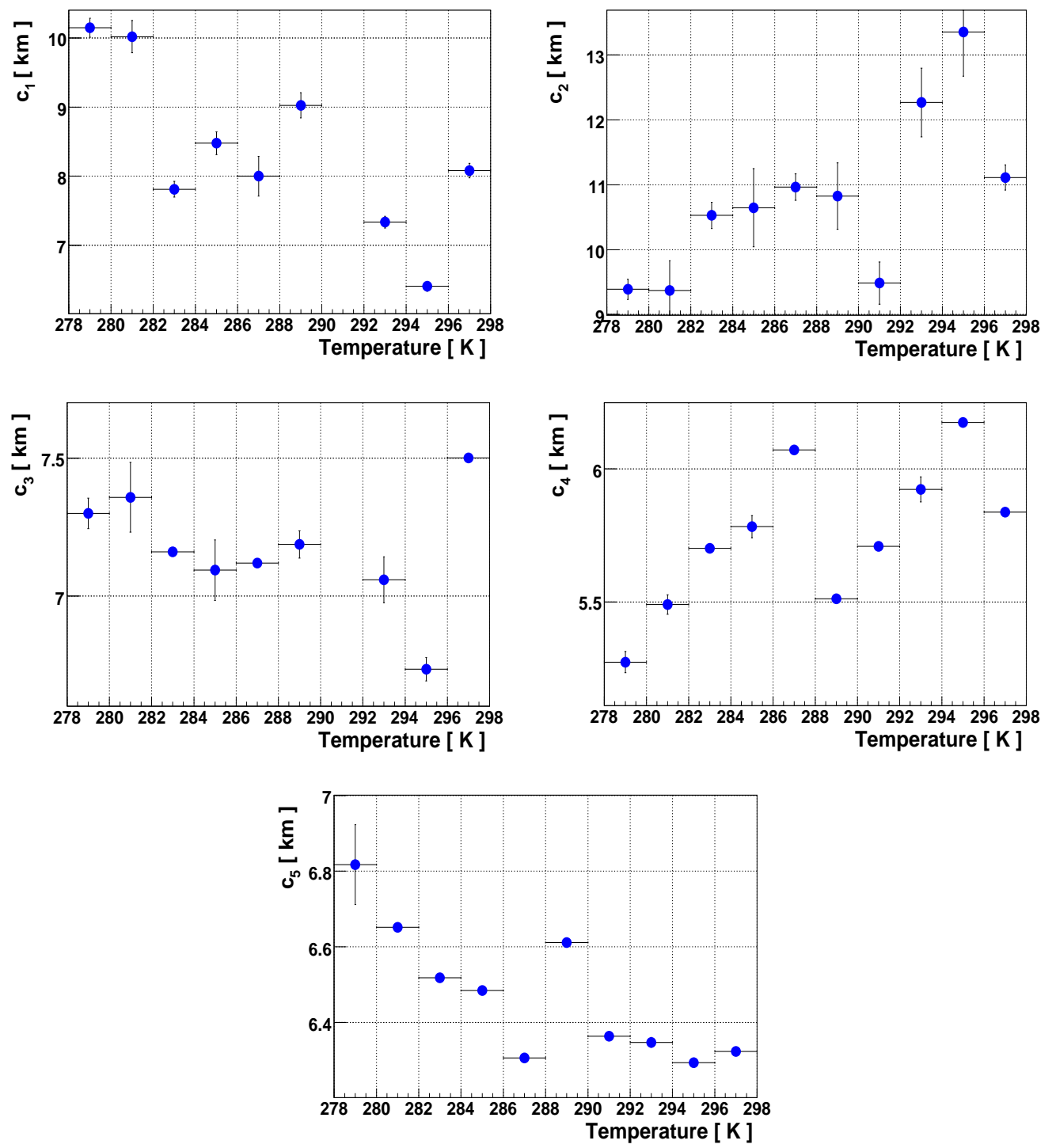

Figura 7.19: Coeficientes $c_{l}(l=1,2,3,4$ y 5) obtenidos en el ajuste GAMMA sobre la base de datos de Dodge City. 


\subsubsection{Base Halley}

La Base Halley se encuentra en las costas del Mar de Wedell en el sector antártico del Reino Unido. La ubicación aproximada de la base es $75^{\circ} 35^{\prime} \mathrm{S}$ $26^{\circ} 34^{\prime}$ O y altura de 30 metros sobre el nivel del mar.

Se utilizaron una serie de perfiles obtenidos entre los meses de junio de 2009 y mayo de 2011. La gran diferencia con los casos anteriores reside en los valores de la temperatura en el nivel del suelo. Como es de esperar en el Polo Sur se suceden muy bajas temperaturas; de hecho, no se encontraron valores por encima de $\operatorname{los} 0^{\circ} \mathrm{C}$. Esto llevó a tomar en este caso, 10 bins de $3 \mathrm{~K}$ cada uno entre los $243 \mathrm{~K}$ y los $273 \mathrm{~K}$. La distribución en los bines puede verse en el Cuadro 7.5.

\begin{tabular}{|c|c|c|}
\hline Bin & T range [K] & Balloons \\
\hline \hline $\mathbf{1}$ & $243 \mathrm{~K}-246 \mathrm{~K}$ & 29 \\
$\mathbf{2}$ & $246 \mathrm{~K}-249 \mathrm{~K}$ & 41 \\
$\mathbf{3}$ & $249 \mathrm{~K}-252 \mathrm{~K}$ & 50 \\
$\mathbf{4}$ & $252 \mathrm{~K}-255 \mathrm{~K}$ & 39 \\
$\mathbf{5}$ & $255 \mathrm{~K}-258 \mathrm{~K}$ & 37 \\
$\mathbf{6}$ & $258 \mathrm{~K}-261 \mathrm{~K}$ & 39 \\
$\mathbf{7}$ & $261 \mathrm{~K}-264 \mathrm{~K}$ & 41 \\
$\mathbf{8}$ & $264 \mathrm{~K}-267 \mathrm{~K}$ & 40 \\
$\mathbf{9}$ & $267 \mathrm{~K}-270 \mathrm{~K}$ & 50 \\
$\mathbf{1 0}$ & $270 \mathrm{~K}-273 \mathrm{~K}$ & 42 \\
\hline
\end{tabular}

Cuadro 7.5: Bines de Temperatura utilizados en Base Halley.

Al igual que en el caso de Dodge City, los ajustes fueron muy exactos, situación que queda reflejada en los gráficos de los Residuos, definidos ahora como

$$
\begin{gathered}
\text { Residuo Halley GAMMA }_{\text {GAM }}(h)=X_{E X P}(h)-X_{\text {Halley GAMMA }}(h) \\
\text { Residuo Halley Linsley }_{(h)}(h) X_{E X P}(h)-X_{\text {Linsley }}(h),
\end{gathered}
$$

donde $X_{\text {Halley }}$ GAMmA $(h)$ representa la predicción de GAMMA usando los valores medios de temperatura en el bin, $X_{E X P}(h)$ el dato del perfil medido en Halley y $X_{\text {Linsley }}(h)$ la consabida función de Linsley. Los anunciados gráficos se observan en las Figuras 7.20, 7.21, 7.22 y 7.23.

Como parte del desarrollo de GAMMA, nuevamente se graficó la dependencia de los coeficientes $c_{l}(l=1,2,3,4,5)$ con la temperatura la nivel del suelo (Figura 7.24). 

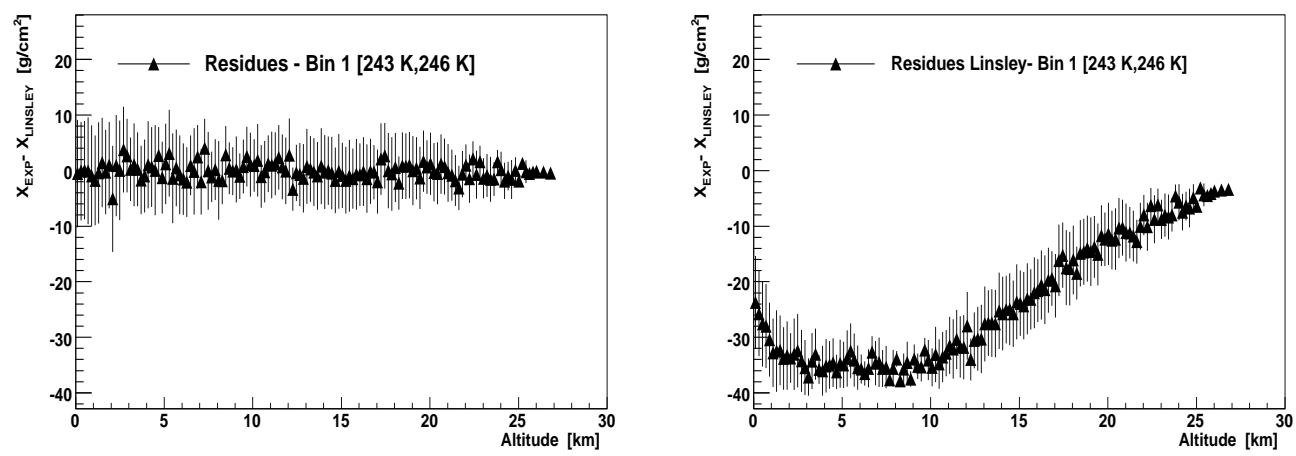

Figura 7.20: Desarrollo del Modelo GAMMA en la Base Halley. Residuos considerando el ajuste de GAMMA (izq) y la función de Linsley (Bin 1).

$\mathrm{Al}$ igual que en el caso anterior, no se puede dejar de notar una serie de comentarios que toman relevancia a partir del estudio presentado.

- En base a los residuos de Linsley, se considera inadecuado el uso del Modelo de Linsley en este sitio. Pese a ser desarollado como un modelo global, es razonable que no sea una buena referencia en este caso, considerando que fue pensado sobre medidas hechas en sitios de latitud media mayormente de Estados Unidos.

- Los residuos son similares en todos los bins. Sin embargo, nuevamente los valores por encima de los $10 \mathrm{~km}$ resultan porcentualmente demasiado altos en los estudios de rayos cósmicos. Esto debe poder solucionarse en el modelo global.

- Los coeficientes obtenidos $c_{l}$ no se corresponden con posibles extrapolaciones al modelo GAMMA desarrollado en Malargüe. Esto abre un campo muy interesante en dirección a un modelo global.

\subsubsection{Comentario final acerca de GAMMA en otros sitios}

Estos primeros resultados son promisorios en la aplicación de GAMMA a otros sitios. La idea central del modelo GAMMA, representar los perfiles a partir de la temperatura al nivel del suelo, ha sido validada nuevamente. Con el objetivo de mejorar el estudio que disminuya las incertezas en estos resultados, se cree que sería necesario un exhaustivo trabajo inicial con la base de datos, además de incluir una diversa cantidad de sitios. 

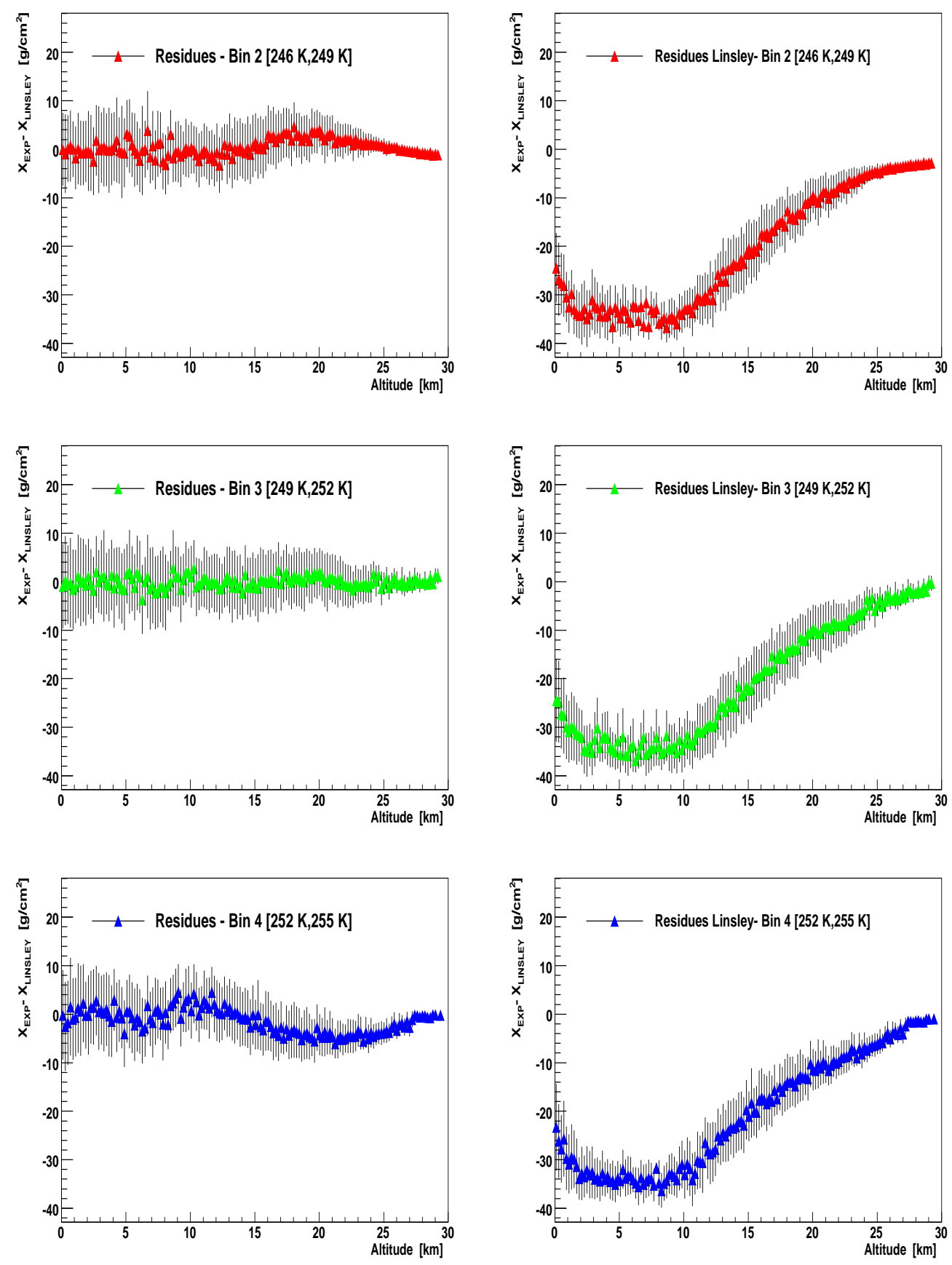

Figura 7.21: Desarrollo del Modelo GAMMA en la Base Halley. Residuos considerando el ajuste de GAMMA (izq) y la función de Linsley (Bins 2, 3 y 4). 

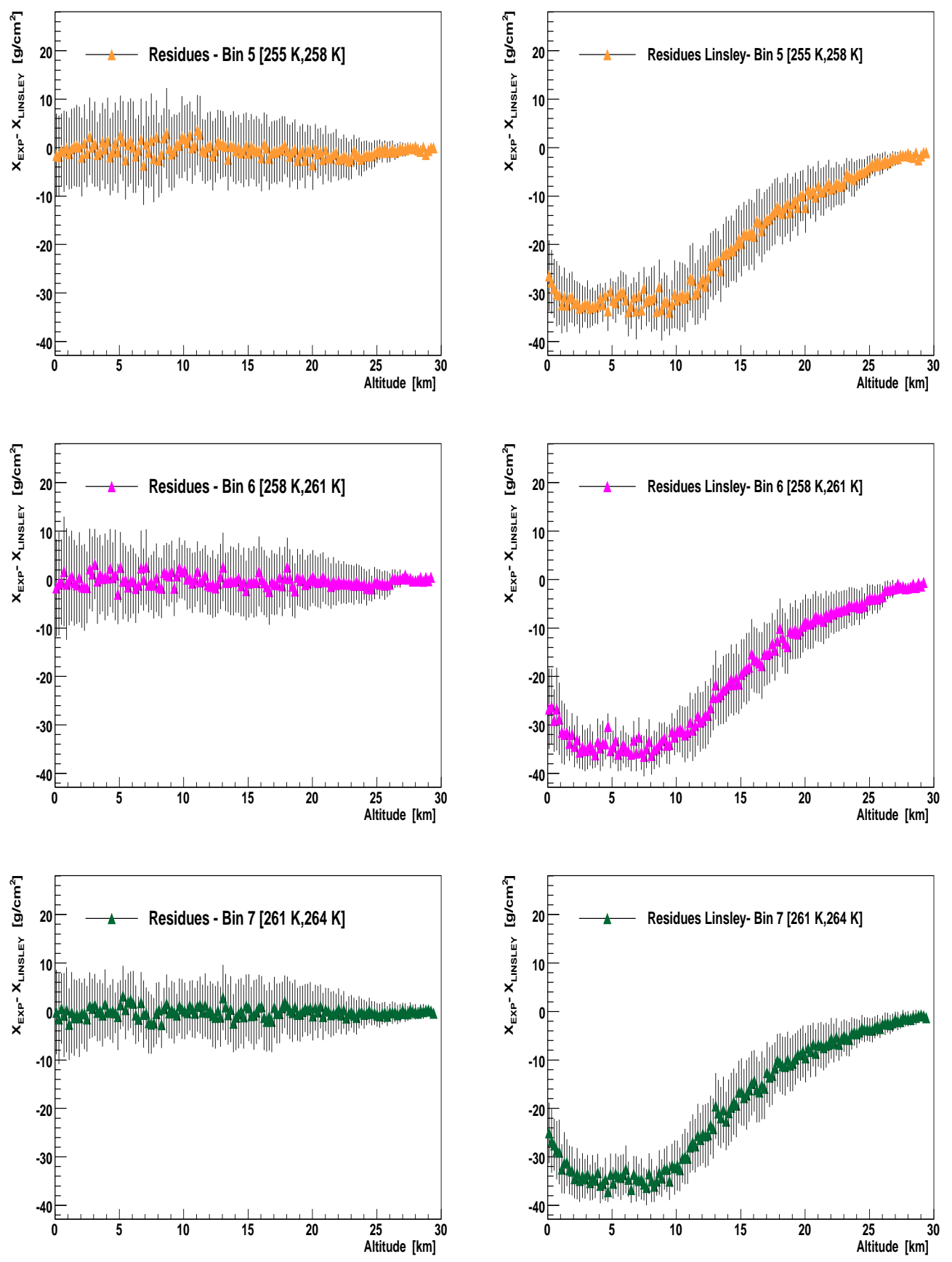

Figura 7.22: Desarrollo del Modelo GAMMA en la Base Halley. Residuos considerando el ajuste de GAMMA (izq) y la función de Linsley (Bins 5, 6 y 7). 

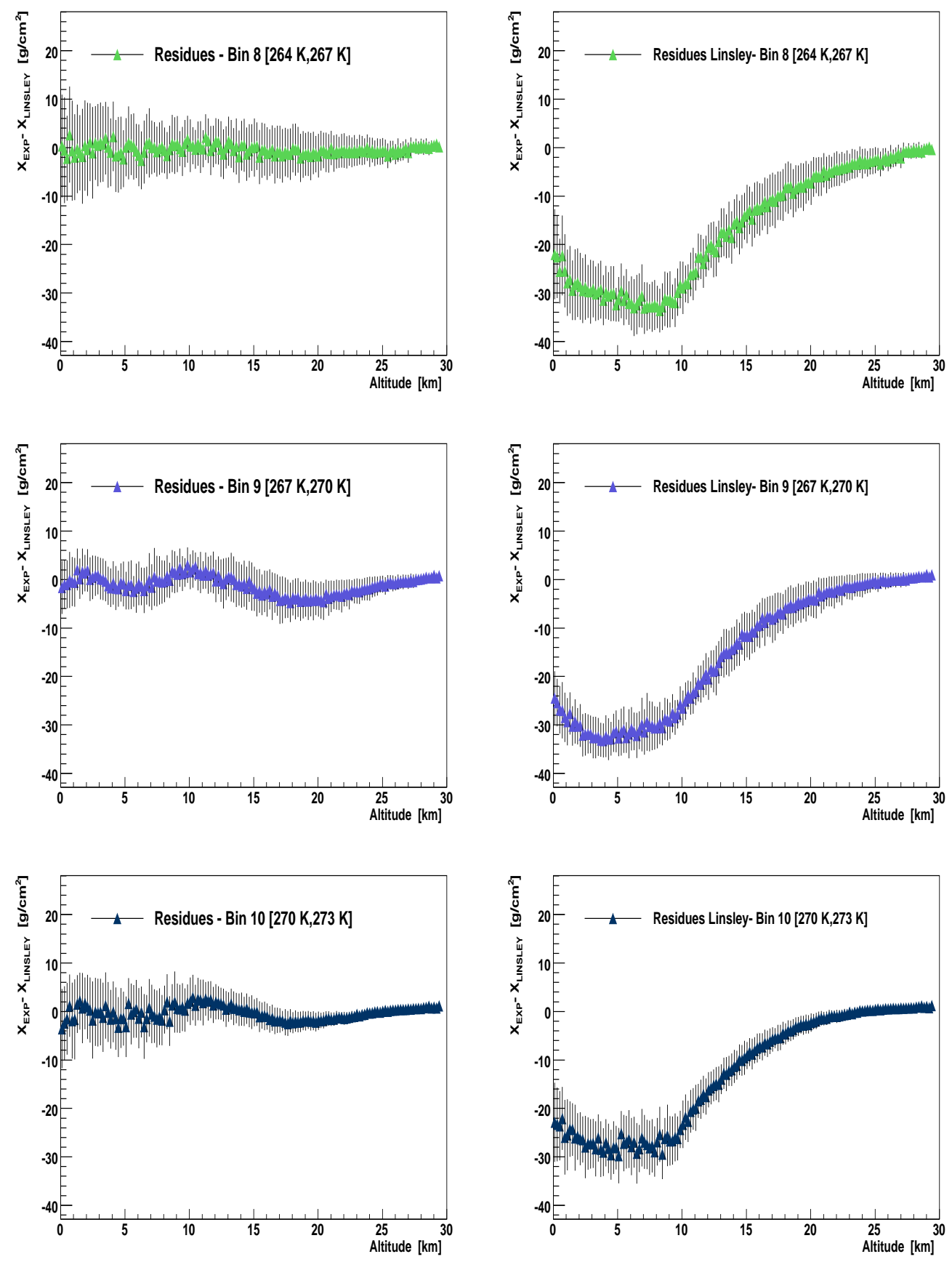

Figura 7.23: Desarrollo del Modelo GAMMA en la Base Halley. Residuos considerando el ajuste de GAMMA (izq) y la función de Linsley (Bins 8, 9 y 10). 

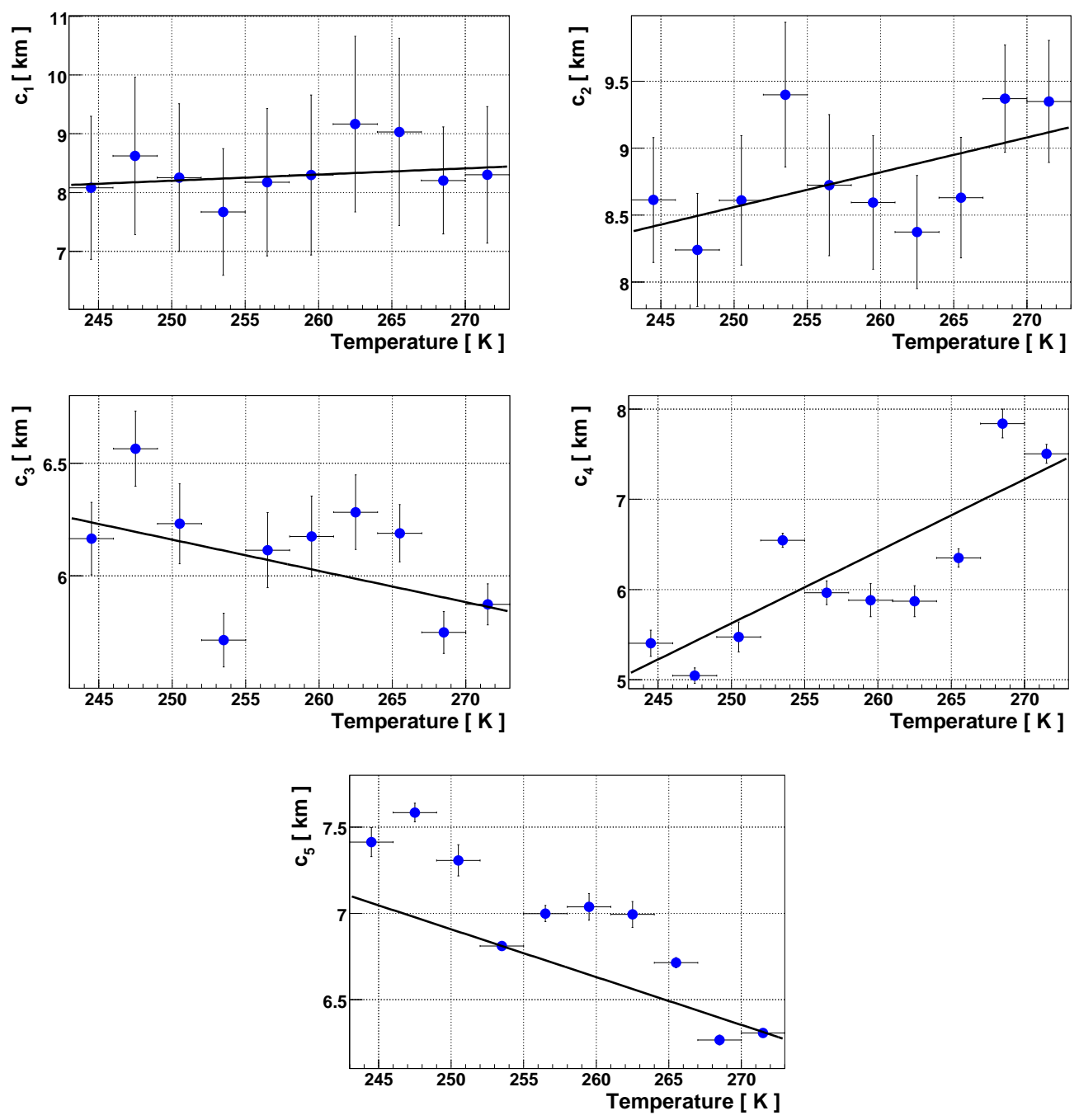

Figura 7.24: Coeficientes $c_{l}(l=1,2,3,4$ y 5) obtenidos en el ajuste GAMMA sobre la base de datos de la Base Halley. 
Por último se quiere dejar sentado que en esta búsqueda del modelo GAMMA de alcance global estimamos que se deber incluir entre sus variables de estudio, a la latitud del sitio, la altura sobre el nivel del mar, la distancia a las costas marítimas y parámetros medios anuales de temperatura, presión y precipitaciones. La posibilidad de un modelo global con bajas incertezas sin duda requerirá de un extenso trabajo en la colección y procesamiento de estos datos de diferentes sitios del planeta. Los buenos resultados obtenidos son alentadores para la continuación en esta dirección. 


\section{Capítulo 8}

\section{GAMMA y los observables}

Este capítulo contiene el estudio vinculado a la influencia debido al perfil de la atmósfera, en algunos observables propios del estudio de cascadas producidas por rayos cósmicos. Los diferentes perfiles estarán representados a partir del modelo GAMMA. Se estudiará particularmente el desarrollo lateral de la lluvia al nivel del suelo, a partir de la función de distribución lateral y el desarrollo longitudinal a partir de la observación de $X_{\max }$ y el perfil del número de partículas cargadas.

\subsection{Distribución lateral al nivel del suelo}

El desarrollo lateral de la cascada fue brevemente descrito en el capítulo 3. Particularmente este análisis estará centrado en la repercusión de los cambios en los perfiles atmosféricos (representados por el modelo GAMMA) en la función de distribución lateral (LDF).

\subsubsection{Más sobre la función de distribución lateral (LDF)}

A modo de introducción al tema, ampliaremos los conceptos vertidos en la sección 3.3 con miras al uso en el Observatorio Auger y en la posible correlación en cambios de la LDF con diferentes perfiles provistos por el modelo GAMMA.

Recordemos que la función de distribución lateral representa la variación de la señal como función de la distancia al eje de la lluvia. Estas señales se producen mayormente por la contribución de los muones y la componente electromagnética de la cascada, compuesta por fotones de radiación gamma, electrones y positrones. Como en las mediciones experimentales no se posee una distribución lateral completa y extensa, sino una serie de señales espa- 
ciadas de acuerdo a las características del arreglo de superficie; la LDF se calcula mediante algunos puntos del plano señal-distancia al eje de la lluvia. Por esta razón una parte vital de la reconstrucción de SD es el uso de un modelo adecuado y robusto de determinar la forma lateral de la cascada.

En el Observatorio Auger, tras varios estudios con datos medidos con el dispositivo SD y utilizando funciones estilo ley de potencias (como fue descrito en la sección 3.3), se optó por una función del tipo Nishimura Kamata Greisen para la descripción lateral. Si bien el desarrollo teórico de estas funciones fue pensado en términos de cascadas puramente electromagnéticas ignorando la contribución muónica; el aporte de esta última componente ha sido incluido modelando las funciones originales [114] [115]. La función seleccionada, actualmente implementada en el ya mencionado software de análisis Offline [121], es

$$
S(r)=S_{1000} \times\left(\frac{r}{1000}\right)^{\beta} \times\left(\frac{r+700}{1700}\right)^{\beta+\gamma}
$$

que se encuentra expresada en términos de $S_{1000}, \beta$ y $\gamma$ y donde $r$ está expresada en metros. La normalización a $S_{1000}$ fue seleccionada considerando que por las características del arreglo sus incertezas debido a las fluctuaciones de las lluvias, cambios en el exponente $\beta$ y efectos de geometría de la lluvia, resultan mínimos en una distancia aproximada de $1000 \mathrm{~m}$.

La parametrización del exponente $\beta$ que está incluida en $\overline{O f f} \underline{\text { line está dada }}$ por

$\beta=\left\{\begin{array}{cc}a+b \times(\sec \theta-1,0) & \sec \theta<1,55 \\ a+b \times(\sec \theta-1,0)+f \times(\sec \theta-1,55)^{2} & 1,55<\sec \theta<3,00 \\ a+b \times\left(0,55-\frac{b}{2 f}\right)+f \times\left(\frac{b}{2 f}\right)^{2} & \sec \theta>3,00\end{array}\right.$

donde $a=-2.26-0.195 \log \mathrm{E}_{\text {est }}, b=0.98$ y $f=-0.295[115]^{1}$. Se ha denominado $\mathrm{E}_{e s t}$ a la energía estimada (no es el resultado final) y su parametrización puede ser encontrada en la referencia [121]. Esta expresión para $\beta$ representada en función de $\log S_{1000}$ para diferentes valores de sec $\theta$ se encuentra representada en la Figura 8.1.

\footnotetext{
${ }^{1}$ Estipulamos la notación utilizada para el logaritmo en base 10 como log
} 


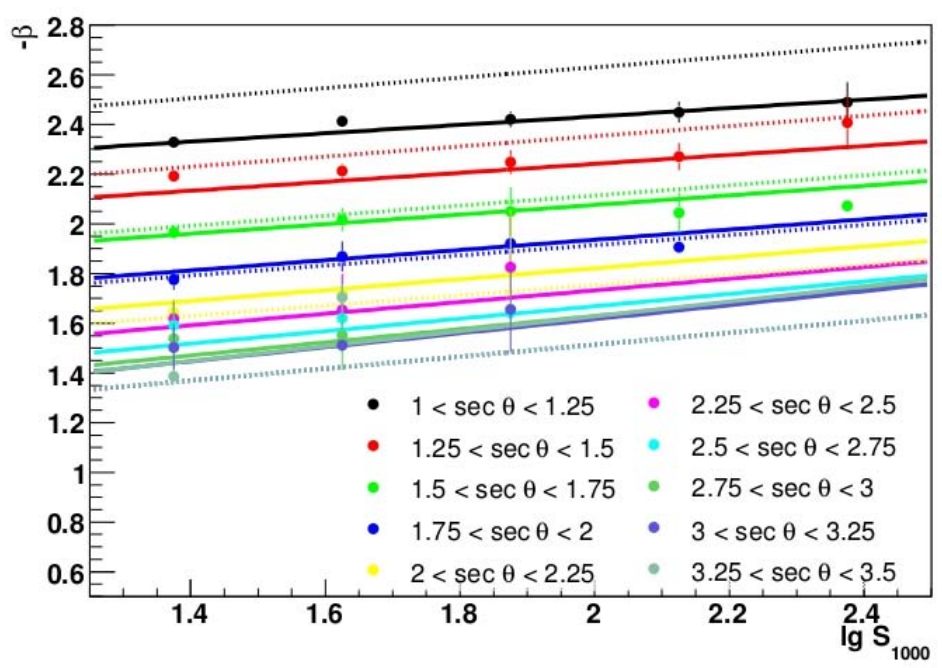

Figura 8.1: Parámetro $\beta$ como función de $\log S_{1000}$ para diferentes valores de $\sec \theta$. La línea punteada muestra la parametrización utilizada en Offline y los puntos representan los valores obtenidos a partir de las reconstrucciones con el mismo software [115].

\subsubsection{Análisis con GAMMA}

En las siguientes secciones describiremos el trabajo hecho estudiando la influencia del modelo de profundidad atmosférica a través de GAMMA y su influencia en la LDF calculada a partir de lluvias simuladas.

\section{Procedimiento}

\section{1 - Librería de lluvias.}

Se simuló una librería de lluvias en AIRES 2.8.7a (versión en desarrollo que incluye como opción al modelo GAMMA) con las siguientes características:

- Partícula primaria: protones de $10^{17} \mathrm{eV}$, de $10^{18} \mathrm{eV}$ y de $10^{19} \mathrm{eV}$.

- Inclinadas un ángulo cenital de $60^{\circ}$.

- Thinning 1.0E-6 Relative

- ThinningWF 0.5

- Injection $115 \mathrm{~km}$

- Ground $1.406 \mathrm{~km}$ 
Se realizó la simulación de 50 lluvias diferentes sobre 16 atmósferas diferentes, 15 de ellas corresponden al modelo GAMMA con diferentes valores de temperatura en el suelo (6 $\mathrm{C}$ a $34 \mathrm{C}$ cada $2 \mathrm{C}$ ) y 1 correspondiente al modelo de Linsley standard. Con estas características, la librería resultó compuesta por un total de 2400 lluvias.

\section{2 - Análisis de los ground particles files.}

Se procedió a hacer el análisis de los archivos ground particles (.grdpcles), es decir los archivos salida de AIRES que contienen toda la información de las partículas que alcanzan el nivel del suelo (tipo de partícula, energía, momento lineal, posición de impacto, etc.). El análisis consistió en leer los archivos .grdpcles de todas las lluvias, realizando las siguientes tareas:

- Selección de datos correspondientes a $\gamma, \mathrm{e}^{+} \mathrm{y} \mathrm{e}^{-}, \mu^{+} \mathrm{y} \mu^{-}$.

- Agrupación de datos en bines de distancia al eje de la lluvia (Ver sección siguiente).

- Estimación de la señal de acuerdo a un software de simulación del detector SD [116].

\section{3 - El bineado en distancia al core.}

El objetivo del bineado es lograr una buena distribución de señal alrededor del core de manera tal de poder calcular LDF con menor incerteza. Considerando que la zona cercana al core posee la mayor cantidad de partículas (tanto que los detectores se saturan de señal), se buscó que los puntos que identifican la señal representen mayores áreas a medida que se alejaran del eje de la lluvia, de manera tal de "colectar" más señal. La manera en la cual se calcula la distancia al eje de la lluvia se encuentra descrita en el Apéndice B.

La proyección del área del suelo en el plano perpendicular al eje de la lluvia fue bineada de acuerdo a regiones anulares con radios en progresión logarítmica de acuerdo con la siguiente expresión

$$
\log r_{\mathrm{j}}=\log r_{\min }+\frac{j}{N} \cdot \log \left(r_{\max } / r_{\min }\right),
$$

donde los valores de $r_{\mathrm{j}}(j=0, . ., N)$ representan los límites de los distintos anillos concéntricos con el eje de la lluvia y el área total es la comprendida entre $r_{\min } \mathrm{y} r_{\max }$. 
Tomando $N=20$ anillos, de radios comprendidos entre $r_{\min }=600 \mathrm{~m} \mathrm{y}$ $r_{\max }=3000 \mathrm{~m}$; se obtuvien los siguientes valores para los radios:

\begin{tabular}{|l|c|}
\hline $\boldsymbol{j}$ & \multicolumn{1}{|c|}{$r_{\mathrm{j}}[\mathbf{m}]$} \\
\hline $\mathbf{0}$ & 600.0 \\
\hline $\mathbf{1}$ & 650.3 \\
\hline $\mathbf{2}$ & 704.8 \\
\hline $\mathbf{3}$ & 763.8 \\
\hline $\mathbf{4}$ & 827.8 \\
\hline $\mathbf{5}$ & 897.2 \\
\hline $\mathbf{6}$ & 972.4 \\
\hline $\mathbf{7}$ & 1053.9 \\
\hline $\mathbf{8}$ & 1142.2 \\
\hline $\mathbf{9}$ & 1237.9 \\
\hline & \\
\hline
\end{tabular}

\begin{tabular}{|c|c|}
\hline $\boldsymbol{j}$ & $r_{\mathrm{j}}[\mathbf{m}]$ \\
\hline $\mathbf{1 0}$ & 1341.6 \\
\hline $\mathbf{1 1}$ & 1454.1 \\
\hline $\mathbf{1 2}$ & 1575.0 \\
\hline $\mathbf{1 3}$ & 1707.9 \\
\hline $\mathbf{1 4}$ & 1851.1 \\
\hline $\mathbf{1 5}$ & 2006.2 \\
\hline $\mathbf{1 6}$ & 2174.3 \\
\hline $\mathbf{1 7}$ & 2356.6 \\
\hline $\mathbf{1 8}$ & 2554.0 \\
\hline $\mathbf{1 9}$ & 2768.0 \\
\hline $\mathbf{2 0}$ & 3000.0 \\
\hline
\end{tabular}

Cuadro 8.1: Radios de los anillos usados en el bineado de estudio de la $L D F$.

El valor en $r$ que representa la señal/área en el bin delimitado por $r_{\mathrm{j}} \mathrm{y}$ $r_{\mathrm{j}+1}$ se denomina $r_{\mathrm{j}+\frac{1}{2}}$ y se calcula mediante

$$
\log r_{\mathrm{j}+\frac{1}{2}}=\log r_{\min }+\frac{\left(j+\frac{1}{2}\right)}{N} \cdot \log \left(r_{\max } / r_{\min }\right)
$$

\section{4 - Estimación de la LDF.}

Los parámetros que definen la LDF se obtienen realizando secuencialmente las siguientes tareas

- Se toman como entradas los datos de los archivos .grdpcles las lluvias (tipo de partícula, energía, distancia al core en bins, número de partículas, señal en VEM), y a partir de las contribuciones de $\gamma, \mathrm{e}^{+} \mathrm{y} \mathrm{e}^{-}, \mu^{+}$ y $\mu^{-}$se calculan la señales totales normalizadas al área.

- Se ajustan los puntos que representan las señales con la distancia al eje de la lluvia para las simulaciones realizadas sobre el perfil atmosférico del modelo de Linsley. El ajuste se realiza mediante la función LDF oficial de Auger 8.1, donde los parámetros libres del ajuste son $S_{1000}$, $\beta$ y $\gamma$. Por simpleza, hemos escrito también la función como

$$
S(r)=S_{1000} \times f(\gamma, \beta, r),
$$


donde

$$
f(\gamma, \beta, r)=\left(\frac{r}{1000}\right)^{\beta} \times\left(\frac{r+700}{1700}\right)^{\beta+\gamma}
$$

con $r$ expresado en metros.

- Con la finalidad de representar adecuadamente las distribuciones ajustadas a los datos obtenidos para cada uno de los perfiles atmosféricos considerados, se normalizan tales distribuciones tomando como unidad de normalización el valor de $S_{1000}$ correspondiente al modelo de Linsley Esta unidad es denominada LES (Linsley Equivalent Signal), y su valor en VEM varía con el valor de la energía del primario.

- Por último se normalizan todas las restantes distribuciones laterales (las correspondientes a las simulaciones sobre perfiles del modelo GAMMA) al LES correspondiente a esa energía y se realizan los ajuste con la LDF, extrayendo los valores de $S_{1000}, \beta$ y $\gamma$.

Sobre las representaciones obtenidas se realizaron dos ajustes, primero dejando libre el parámetro $\gamma$ y después fijándolo a 0 (esta elección es la usual en el trabajo con datos ya que se poseen pocos datos para realizar el ajuste). Esto influye muy levemente (menos del $0.2 \%$ ) en algunos de los valores de $S_{1000}$ obtenidos. Estos valores, para el caso del perfil de Linsley se encuentran detallados en el Cuadro 8.2 identificando los utilizados en la normalización LES, mientras que para los otros perfiles se presentan más adelante en la figura 8.4. Allí se ilustra el ajuste con $\gamma$ nulo, ya que el caso con $\gamma$ libre produce resultados que no presentan diferencias apreciables en las gráficas y por lo tanto los mismos no han sido incluidos en esa figura.

\begin{tabular}{|c|c|c|}
\hline $\begin{array}{c}\text { Energía } \\
{[\mathbf{e V}]}\end{array}$ & $\begin{array}{c}\text { Fit con } \gamma \text { libre } \\
\mathbf{1} \text { LES }[\text { VEM] }\end{array}$ & $\begin{array}{c}\text { Fit con } \gamma=\mathbf{0} \\
\mathbf{1} \text { LES }[\text { VEM] }\end{array}$ \\
\hline $10^{17}$ & $5.535 \times 10^{-2}$ & $5.534 \times 10^{-2}$ \\
\hline $10^{18}$ & $5.264 \times 10^{-1}$ & $5.264 \times 10^{-1}$ \\
\hline $10^{19}$ & $5.780 \times 10^{0}$ & $5.765 \times 10^{0}$ \\
\hline
\end{tabular}

Cuadro 8.2: Valores de LES obtenidos para las diferentes energías estudiadas. El LES representa el valor de $S_{1000}$ obtenido para las lluvias desarrolladas sobre la atmósfera del modelo de Linsley.

A modo de ejemplo, se muestran los histogramas correspondientes a las componentes electromagnéticas y muónicas para lluvias originadas por protones de $10^{17}$ y $10^{18}$ eV y desarrolladas sobre atmósferas caracterizadas por temperatura al nivel del suelo con valores $10 \mathrm{C}$ y $20 \mathrm{C}$ (Figura 8.2). 

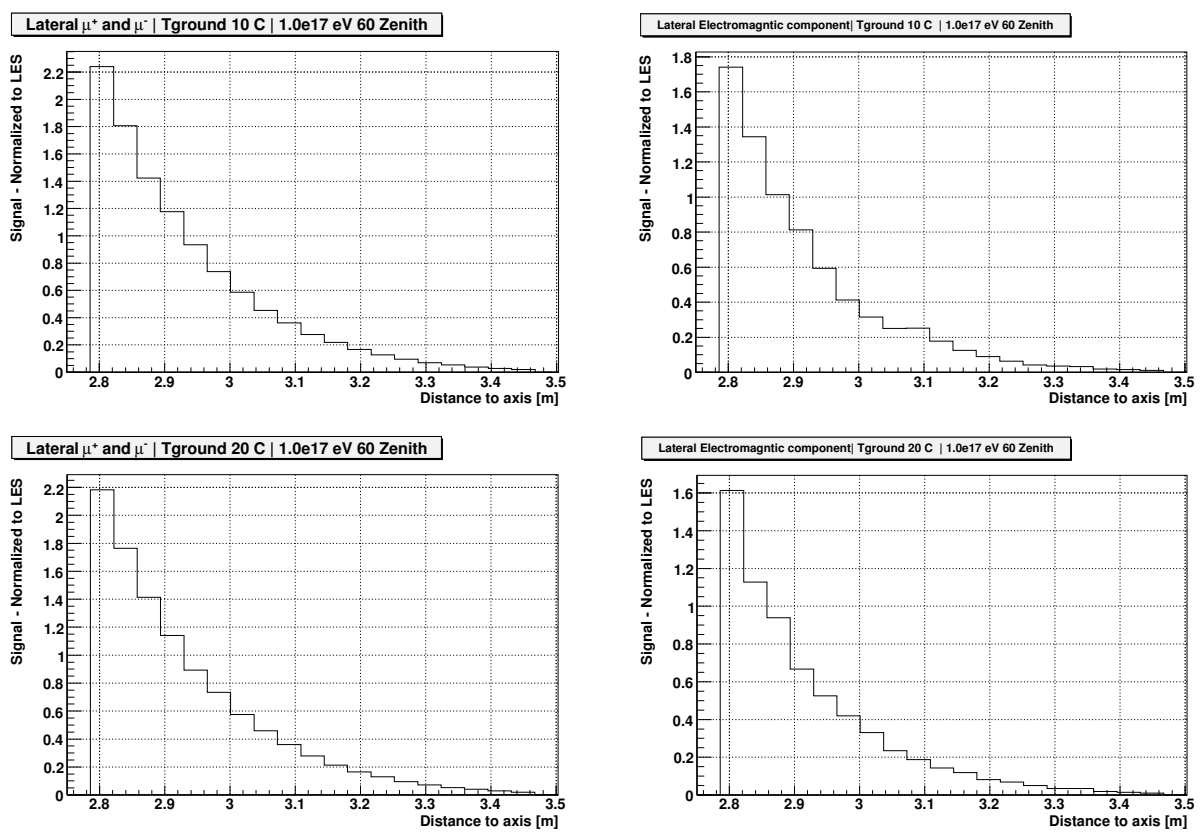

Figura 8.2: Ejemplos de histogramas promedios correspondientes a señales generadas por muones y la componente electromagnética para lluvias inducidas por protones, con una inclinación de $60^{\circ}$ y desarrolladas en atmósferas GAMMA de temperaturas $10 \mathrm{C}$ y $20 \mathrm{C}$. 


\section{5 - Estudio de la LDF.}

Como fue planteado anteriormente se realizaron dos ajustes con la LDF, ambos en el mismo rango de distancia al core (600 a $3000 \mathrm{~m}$ ) pero uno dejando libre el parámetro $\gamma$ en el ajuste, y otro igualándolo a 0. Los ajustes resultan prácticamente independientes de la inclusión del parámetro $\gamma$ en la función, marcando que el peso crítico en el ajuste está sobre el parámetro $\beta$. Como lo usual en el trabajo con datos es considerar $\gamma=0$, nos referiremos ahora a los ajustes obtenidos de este último modo.

Todos estos ajustes resultan muy precisos para todas las temperaturas y energías, por lo que sólo se muestran algunos de ellos (Figuras 8.3). Asimismo, como también fue anticipado, los ajustes de los desarrollos laterales, no determinaron (prácticamente) diferentes valores de LES, ni diferentes valores y dispersión de $S_{1000}$.

Luego se compararon los valores de $S_{1000}$ obtenidos sobre el ajuste de los diferentes desarrollos laterales (Figuras 8.4). Naturalmente el más cercano al standard de 1 LES corresponde al ajuste sobre la atmósfera Linsley y en las gráficas mencionadas es el que se ubica con temperatura $36 \mathrm{C}$.

Si bien la precisión en los ajustes no resulta afectada por la inclusión del parámetro $\gamma$, posteriormente se estudió su influencia en la estructura funcional de la LDF (por ejemplo observar si se detectan oscilaciones, o si siempre es mayor o menor que la LDF de Linsley). Con este motivo se retomaron los ajustes con $\gamma$ libre y $\gamma$ fijo a 0 , y se graficó

$$
\frac{f\left(\gamma_{\text {Tground }}, \beta_{\text {Tground }}, r\right)}{f\left(\gamma_{\text {Linsley }}, \beta_{\text {Linsley }}, r\right)}=\frac{\left(\frac{r}{1000 m}\right)^{\beta_{\text {Tground }}} \times\left(\frac{r+700 m}{1700 m}\right)^{\beta_{\text {Tground }}+\gamma_{\text {Tground }}}}{\left(\frac{r}{1000 m}\right)^{\beta_{\text {Linsley }}} \times\left(\frac{r+700 m}{1700 m}\right)^{\beta_{\text {Linsley }}+\gamma_{\text {Linsley }}}}
$$

para las diferentes funciones obtenidas en los distintos ajustes.

Las estructuras observadas en el caso de $\gamma$ libre resultaban dispares y nada uniformes. De hecho esa fue la motivación en su momento para realizar el ajuste con $\gamma$ fijo y nulo. Los gráficos muestran que en este último caso, al depender de un sólo parámetro exponencial, todas las funciones tienen un comportamiento similar entre sí y también con respecto al ajuste realizado sobre la atmósfera de Linsley (Figuras 8.5, 8.6 y 8.7). Es decir, si las funciones eran mayores (menores) que la de Linsley para $\mathrm{R}<1000 \mathrm{~m}$, luego se observaban mayores (menores) que la de Linsley para $\mathrm{R}>1000 \mathrm{~m}$.

Antes de avanzar en el estudio, se destacarán una serie de puntos obtenidos de las figuras anteriores. Es evidente que en general el ajuste del desarrollo lateral de las lluvias desarrolladas en la atmósfera de Linsley promueve valores de $S_{1000}$ por encima de los obtenidos sobre lluvias desarrolladas sobre las atmósferas provistas por el modelo GAMMA (Figuras 8.4). Esto resulta 

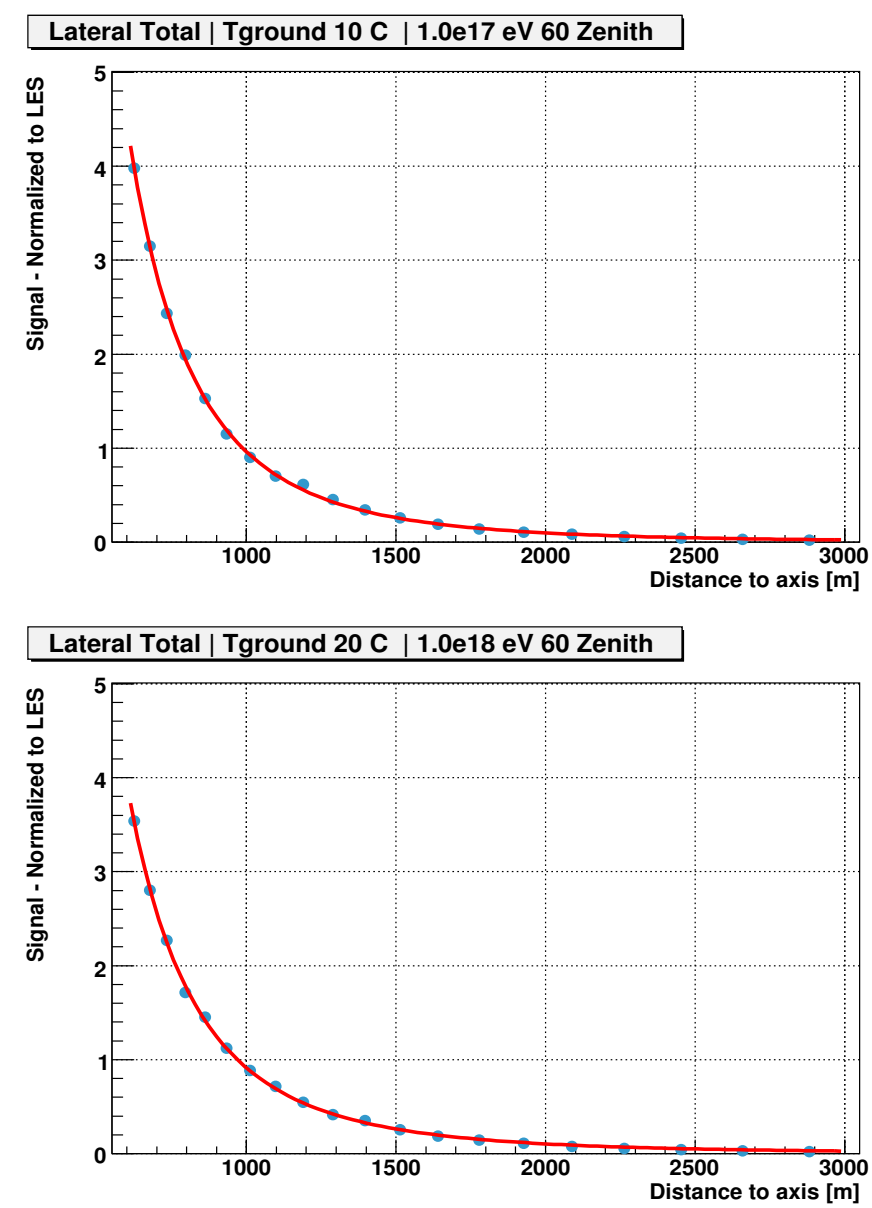

\begin{tabular}{|l|l|}
\hline Lateral Total | Tground $30 \mathrm{C} \mid 1.0 \mathrm{e} 19 \mathrm{eV} 60$ Zenith \\
\hline
\end{tabular}

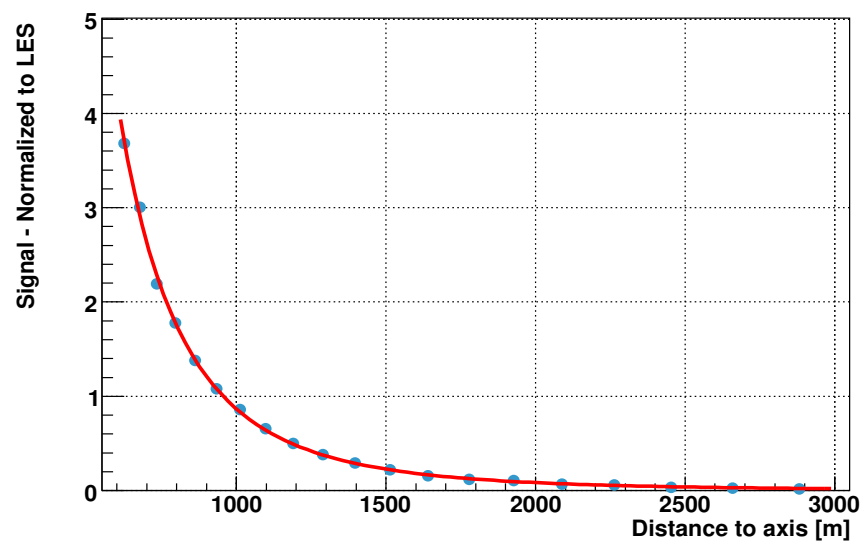

Figura 8.3: Ejemplos del ajuste de la función de distribución lateral para los casos de energía del primario $10^{17}$ y la atmósfera GAMMA de $10 \mathrm{C}$ (arriba), $10^{18}$ y la atmósfera GAMMA de $20 \mathrm{C}$ (centro) y $10^{19}$ y la atmósfera GAMMA de 30 C (abajo). Cada punto representa el promedio de señal, a esa distancia al eje, de 50 lluvias desarrolladas en esa atmósfera GAMMA. 


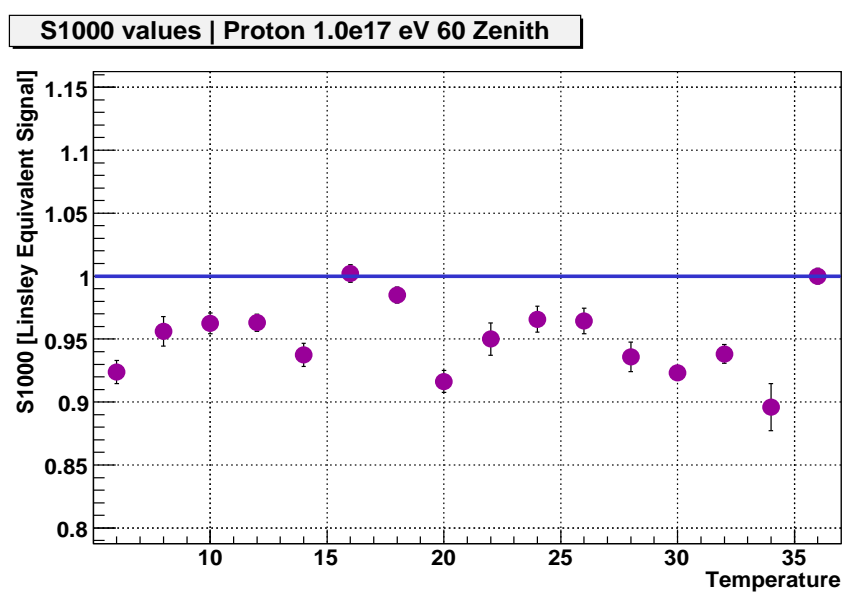

S1000 values | Proton 1.0e18 eV 60 Zenith

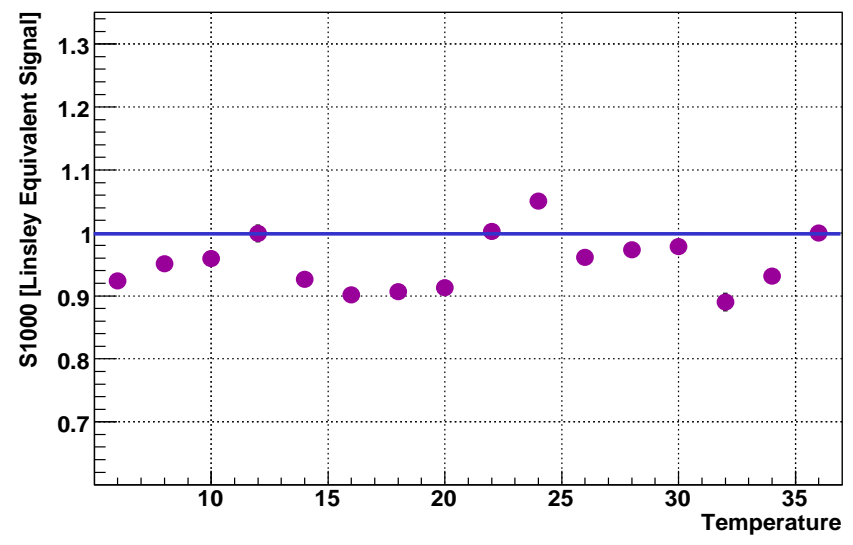

S1000 values | Proton 1.0e19 eV 60 Zenith

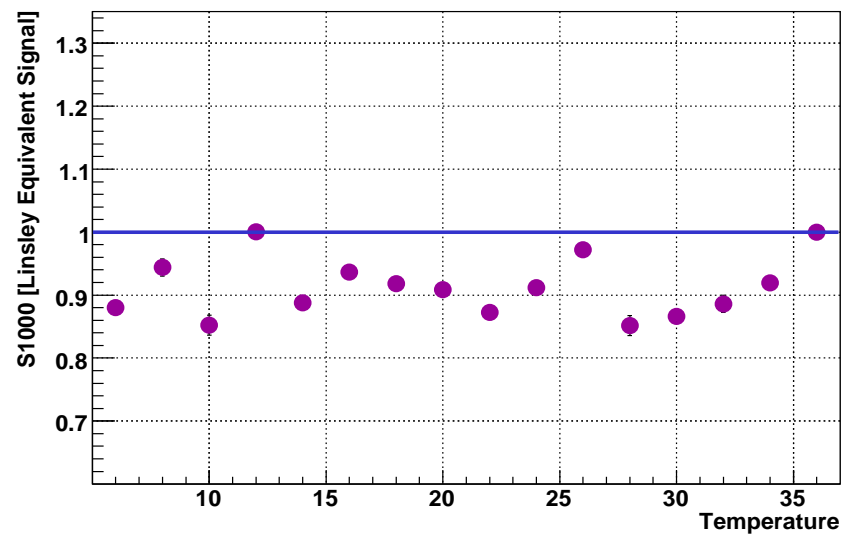

Figura 8.4: Valores de $S_{1000}$ obtenidos con la función de ajuste fijando $\gamma=$ 0 para primarios con energía $10^{17}$ (arriba), $10^{18}$ (centro) y $10^{19} \mathrm{eV}$ (abajo). El valor correspondiente al ajuste en la atmósfera de Linsley se encuentra ubicado en los 36 C. 

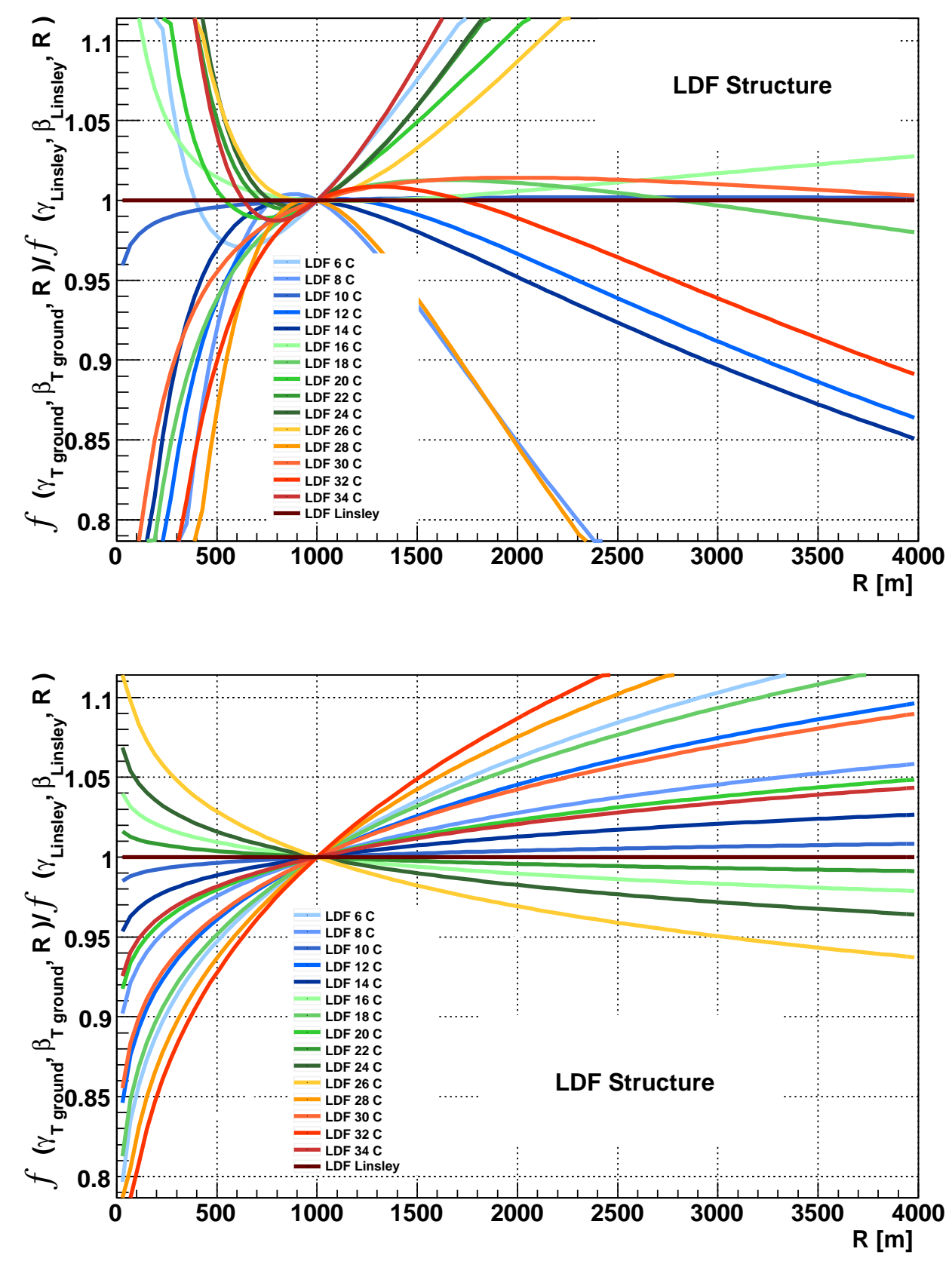

Figura 8.5: Primario $10^{17} \boldsymbol{e} \boldsymbol{V}$ : Estructura de las LDF en comparación con la LDF obtenida ajustando sobre la atmósfera de Linsley, utilizando la función de ajuste incluyendo $\gamma$ libre (arriba) y fijando $\gamma=0$ (abajo). 

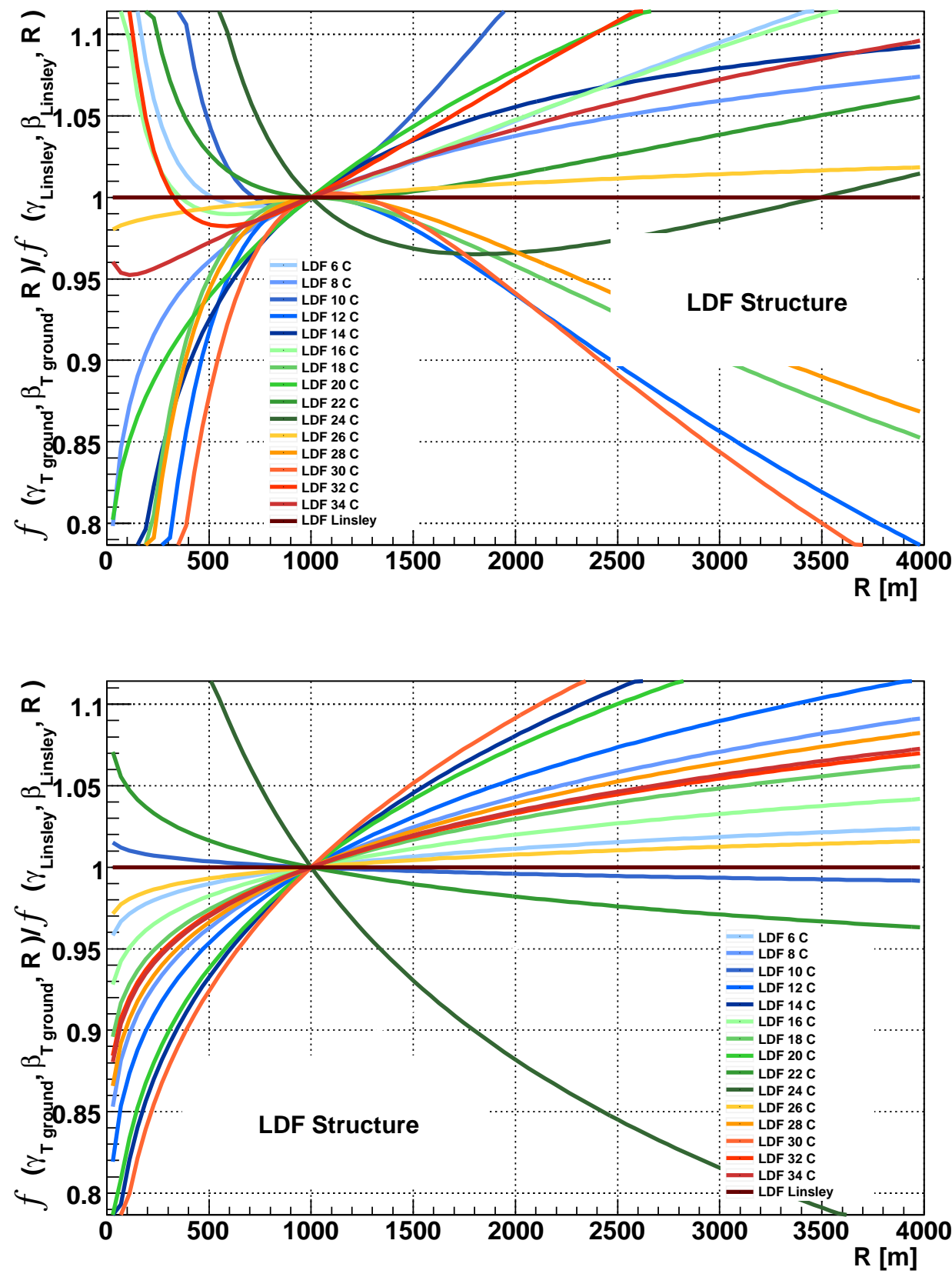

Figura 8.6: Primario $10^{18}$ e $\boldsymbol{V}$ : Estructura de las LDF en comparación con la LDF obtenida ajustando sobre la atmósfera de Linsley, utilizando la función de ajuste incluyendo $\gamma$ libre (arriba) y fijando $\gamma=0$ (abajo). 

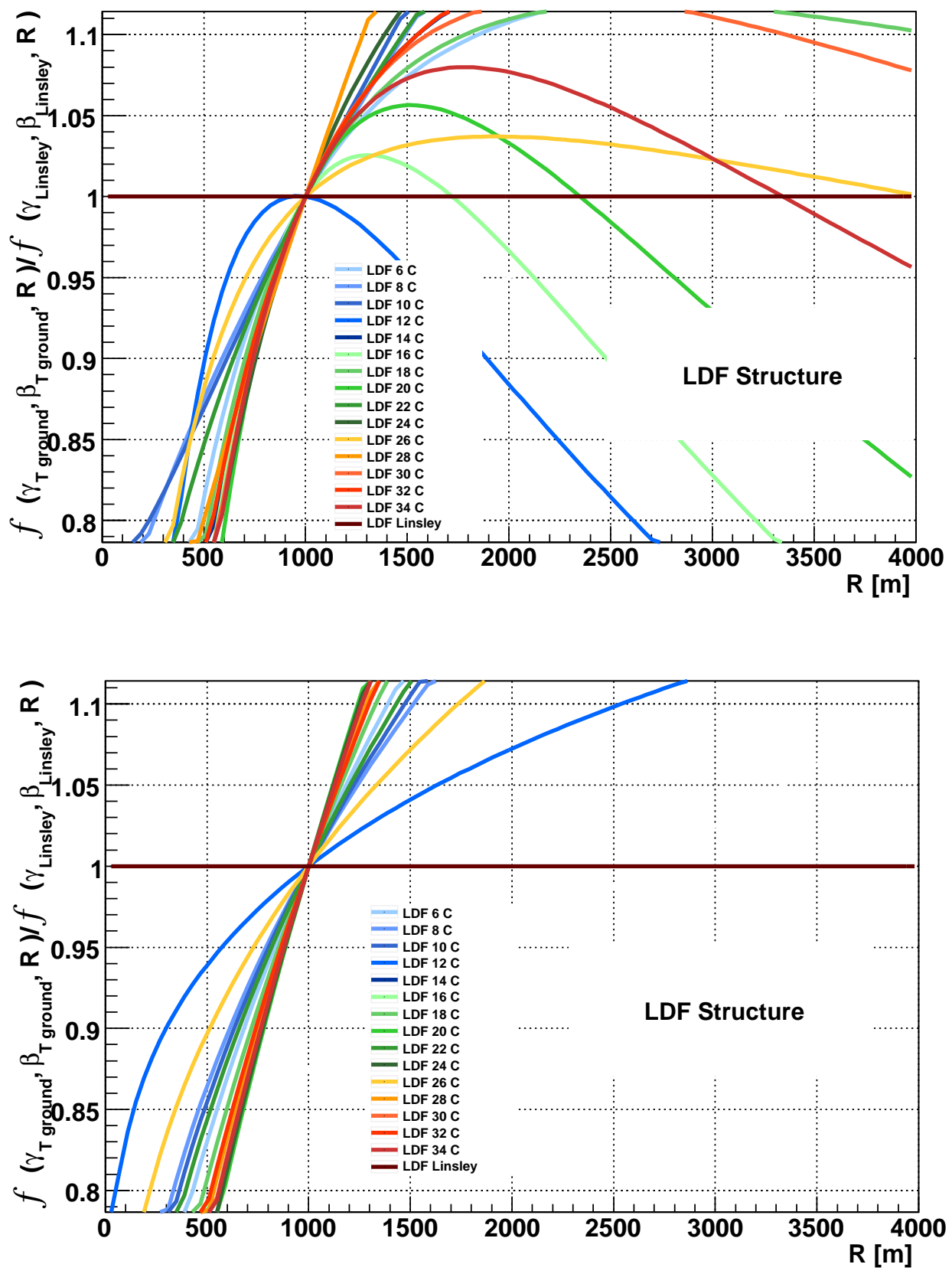

Figura 8.7: Primario $10^{19}$ e V: Estructura de las LDF en comparación con la LDF obtenida ajustando sobre la atmósfera de Linsley, utilizando la función de ajuste incluyendo $\gamma$ libre (arriba) y fijando $\gamma=0$ (abajo). 
independiente de la energía y de haber usado en el ajuste funciones con el exponente $\gamma$ nulo o libre. Es destacable los valores encontrados en las diferencias (hasta $11.5 \%$ en los casos de energías $10^{17} \mathrm{eV}$ y $10^{18} \mathrm{eV}$, y hasta $15 \%$ en los casos de energía $10^{19} \mathrm{eV}$ ) y que no se detecta una estructura en las diferencias (es decir que no se observa que la diferencia aumente o disminuya de acuerdo se trate de lluvias desarrolladas en atmósferas de mayor o menor temperatura).

El incluir el parámetro $\gamma$ como no nulo en la función de distribución lateral no repercute evidentemente en la precisión del ajuste. Sin embargo en relación a la estructura de las funciones $S(r)$, la influencia del valor de $\gamma$ claramente no es despreciable (figuras 8.5, 8.6 y 8.7). El ajuste resulta muy sensible a variaciones que repercuten en valores de $\gamma$ siempre pequeños pero positivos y negativos, que cambian sustancialmente la estructura funcional. Se observa al fijar $\gamma=0$ que el valor $R=1000 \mathrm{~m}$ resulta un valor crítico en la relación 8.6 , ya que $f\left(\gamma_{\text {Tground }}=0, \beta_{\text {Tground }}, r\right)$ (además de ser coherente entre todas las atmósferas GAMMA y para todas las energías) resulta ser mayor que $f\left(\gamma=0, \beta_{\text {Linsley }}, r\right)$ en la gran mayoría de los casos para distancias mayores a $1000 \mathrm{~m}$ y menor para distancias menores a $1000 \mathrm{~m}$. Si bien para descartar el uso del parámetro $\gamma$ se requiere un estudio más profundo centrado exclusivamente en los ajustes con la función de distribución lateral, no hemos encontrado en este estudio un mayor sentido a su inclusión en la función de ajuste.

El haber utilizado en el ajuste los valores $S_{1000}$ normalizados, dificulta la comparación directa con datos del Observatorio Auger. Sin embargo se corrobora que los parámetros alcanzados que daban forma a la estructura funcional son consistentes con los datos. Esto puede observarse de las figuras 8.8 realizadas sobre los set de lluvias descritos, con la figura 8.9 realizada sobre datos de lluvia donde se estimaba la energía del primario entre $10^{18,5}$ eV y $10^{19} \mathrm{eV}$; y el ángulo de inclinación del eje de la lluvia se encontraba en el rango $1.4<\sec \theta<1.6$. Si bien no son completamente comparables porque los rangos de energía e inclinación son diferentes, queremos destacar la coincidencia en los valores y tendencia en el rango común: $2.4<\log R<$ 3.4 .

Debemos acentuar algo también esperable, de las figuras 8.8 se observan gráficas muy similares para todas las atmósferas y la no dependencia de la energía.

Por último observemos que los valores de $\beta$ obtenidos son prácticamente coincidentes con los estipulados para el uso del software $\overline{\text { Offline. Como }}$ además era esperable, son independientes de la energía. Los valores de $\beta$ se encuentran en el Apéndice C clasificados de acuerdo a su energía y atmósfera GAMMA y los valores utilizados en $\overline{O f f}$ line se desprenden de la Figura 8.1. 

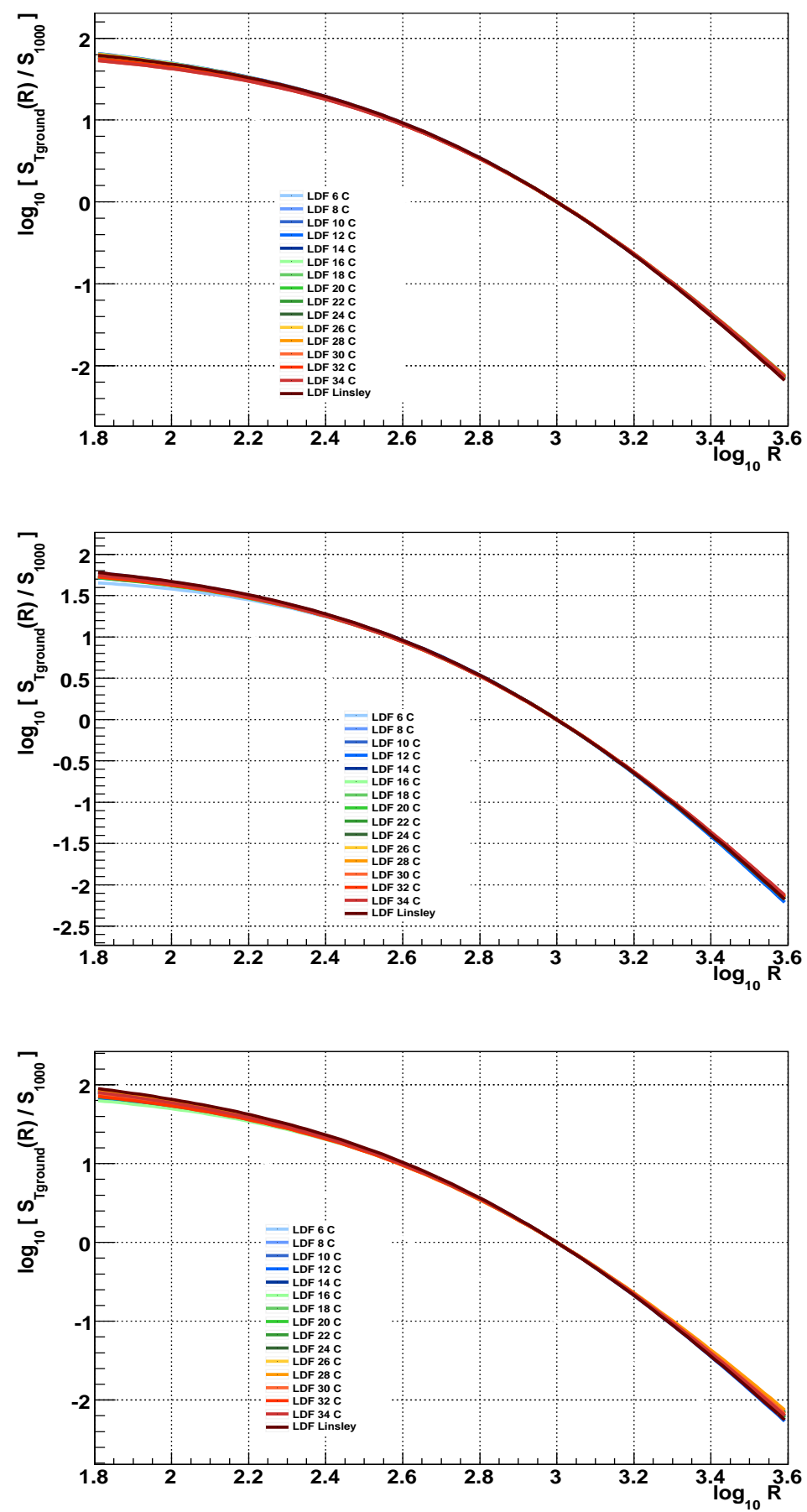

Figura 8.8: Distribución lateral promedio sobre lluvias simuladas. Rango estudiado: $\sec \theta=2$ y energía del primario $10^{17} \mathrm{eV}$ (arriba), $10^{18} \mathrm{eV}$ (centro) y $10^{19}$ eV (abajo). 


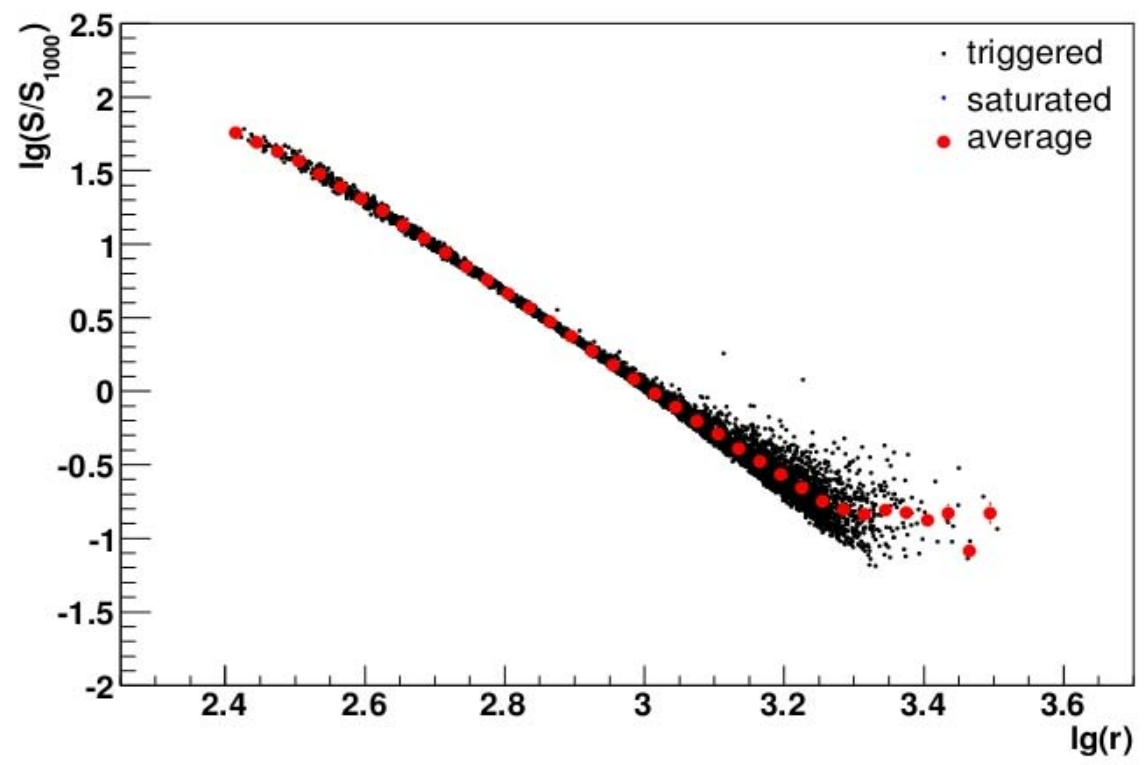

Figura 8.9: Distribución lateral promedio de datos de Auger. Rango estudiado: $1.2<\sec \theta<1.4$ y $18.5<\log (E / 1 \mathrm{eV})<19.0$, incluye los datos correspondientes a estaciones con medidas y el perfil promedio. 


\subsection{Desarrollo longitudinal}

De manera similar a lo explicado al comienzo de la sección anterior, el desarrollo longitudinal de las cascadas también fue expuesto en el capítulo 3. En esta sección nos enfocaremos en estudiar la variación en diferentes atmósferas de dos parámetros vinculados a él: la profundidad atmosférica a la cual coexisten el mayor número de partículas $\left(X_{\max }\right)$, y el perfil longitudinal del número de partículas cargadas.

\subsubsection{Modelos atmosféricos y Desarrollo Longitudinal}

Hemos planteado que en cascadas de rayos cósmicos el número de partículas cargadas como función de la profundidad atmosférica está estrechamente relacionado al tipo de partícula primaria y su energía. Para una dada energía, los protones producen cascadas que se desarrollan, en promedio, más profundamente en la atmósfera que las originadas por núcleos como primarios.

Es por eso que el valor de $X_{\max }$ está correlacionado con la masa de la partícula primaria. Sin embargo la naturaleza estocástica del proceso de producción de partículas en la cascada deriva en grandes fluctuaciones lluvia a lluvia. Por otro lado, el tamaño de las fluctuaciones depende de la masa también, por lo que ambos, el valor de $X_{\max }$ y el ancho de su distribución, son pistas importantes para dilucidar la composición de la partícula primaria.

Claramente esto implica entonces un pormenorizado control de las medidas experimentales referidas a estos parámetros, en particular en las técnicas de medición mediante fluorescencia, la más utilizada en los estudios de desarrollo longitudinal para primarios de energías mayores a $10^{17} \mathrm{eV}$. Recordemos que esta técnica se basa en el hecho de que las partículas que atraviesan la atmósfera excitan las moléculas de Nitógeno y su desexcitación resulta en emisión de luz en el rango de fluorescencia, y el factor de producción de fluorescencia es proporcional a la energía depositada por las partículas de la cascada y que generan la ionización local. Este hecho deriva justamente en que el perfil longitudinal de la lluvia puede ser reconstruido del perfil de luz de fluorescencia observado.

Vinculado a esta problemática de determinación de perfiles de desarrollo longitudinal, no debemos olvidar que los detectores de fluorescencia operan en un campo visual angular fijo y el rango de altitud equivalente a este campo visual depende de la distancia del eje de la lluvia al telescopio. El número de partículas en la cascada en un instante de tiempo depende de la cantidad de materia que se haya atravesado $(X)$, pero esta dependencia en situaciones experimentales se observa en términos de la altura $h$. Esto implica un conocimiento preciso de la correlación entre la altura $h$ medida y la profundidad 
atmosférica $X$, correlación dada por el modelo de profundidad atmosférica. Debido a que las expresiones funcionales de los modelos atmosféricos Linsley, NMMM y GAMMA están expresadas en término de profundidad atmosférica vertical $\left(X_{\text {vertical }}\right)$, la vinculación con la profundidad atmosférica en la dirección del eje de la lluvia inclinada un ángulo cenital $\theta$ puede ser aproximada mediante

$$
X_{\text {inclinado }}=X_{\text {vertical }} / \cos \theta,
$$

donde $X_{\text {inclinado }}$ representa ahora la cantidad efectiva de materia atravesada en una lluvia inclinada un ángulo $\theta<60^{\circ}$. Debido a este punto, los análisis de desarrollo longitudinal dependen fuertemente del ángulo cenital de la lluvia.

\subsubsection{GAMMA y $X_{\max }$}

Como primer estudio se observó, mediante la aplicación del modelo GAMMA, la influencia del modelo de profundidad atmosférica en el parámetro $X_{\max }$. La manera en la que fue hecho es similar a lo anterior: se simularon con AIRES una serie de lluvias que se desarrollaban en diferentes atmósferas. En particular, el set de lluvias utilizado en este caso tenía las siguientes características

- Partículas primarias: protones y núcleos de hierro.

- Energía del primario: $10^{17} \mathrm{eV}, 5 \times 10^{17} \mathrm{eV}, 10^{18} \mathrm{eV}, 5 \times 10^{18} \mathrm{eV}$, y $10^{19}$ $\mathrm{eV}$.

- Eje de la lluvia vertical: ángulo cenital $0^{\circ}$.

- Modelo Atmosférico: GAMMA con 15 temperaturas diferentes al nivel del suelo (tomadas cada $2 \mathrm{C}$ entre $6 \mathrm{C}$ y $34 \mathrm{C}$ ) y Linsley.

- Thinning 1.0E-6 Relative

- ThinningWF 0.5

- Injection $115 \mathrm{~km}$

- Ground $1.406 \mathrm{~km}$

Considerando que para cada una de estas combinaciones se simularon 100 lluvias, el conjunto total analizado es de 16000 lluvias.

Utilizando las funciones del programa AIRES, se obtienen los valores de $X_{\max }$ y $N_{\max }$ de cada una de las lluvias. Los valores promedios de $X_{\max }$ para 
cada combinación Primario-Energía-Atmósfera se encuentran en las siguientes figuras, donde debe aclararse que la línea representa el valor del promedio de Linsley y el sombreado de color representa el ancho correspondiente a incluir la desviación standard. Para que sirva de comparación, las gráficas de $X_{\max }$ fueron hechas a la misma escala, algo no realizado con $N_{\max }$ debido a los valores involucrados.

A partir de los gráficos de $X_{\max }$ en función de la temperatura al nivel del suelo utilizada en cada atmósfera GAMMA, se observa que todos los valores medios de $X_{\max }$ se encuentran prácticamente en el mismo rango de incerteza (Figuras 8.10 a 8.14). Se estima entonces que si existiera alguna tendencia que permita discriminar entre los casos con atmósferas GAMMA de temperaturas bajas, medias y altas, no puede observarse debido a que estaría contenida dentro de las fluctuaciones lluvia a lluvia.

En definitiva lo que sucede es que las lluvias se desarrollan todas dentro del mismo gramaje. Es decir que de acuerdo a las simulaciones, una vez definido el tipo de partícula primaria y su energía, la cantidad de materia atravesada es independiente del modelo atmosférico. Este resultado es esperable pero confirma que la parametrización del modelo GAMMA no introduce una variación en este punto.

A modo de ejemplo se muestra también los valores de $N_{\max }$ alcanzados para las diferentes atmósferas (Figura 8.15). Todos los valores promedios de $N_{\text {max }}$ alcanzados, se encuentran contenidos dentro del nivel de incerteza de Linsley. Este resultado resulta lógico y coherente con la idea de que el número máximo de partículas no cambie en valor medio en las diferentes atmósferas (para primario, energía, y sec $\theta$ fijos). Además, como era de esperar tanto los valores como los incertezas son razonables para la elección de tipo de primario, energía, inclinación del eje de la lluvia y modelo hadrónico.

Podemos concluir que no se han observado, al menos mediante los parámetros $X_{\max }$ y $N_{\max }$, una influencia destacable del modelo atmosférico GAMMA en el estudio del desarrollo longitudinal de la lluvia, y en todos los casos se reproducen valores similares al modelo de Linsley. Sin embargo, como cada atmósfera GAMMA plantea una diferente parametrización para la profundidad atmosférica, los similares valores de $X_{\max }$ representan en realidad diferentes valores en altura. El estudio del desarrollo longitudinal vinculado a este punto se encuentra la siguiente sección. 

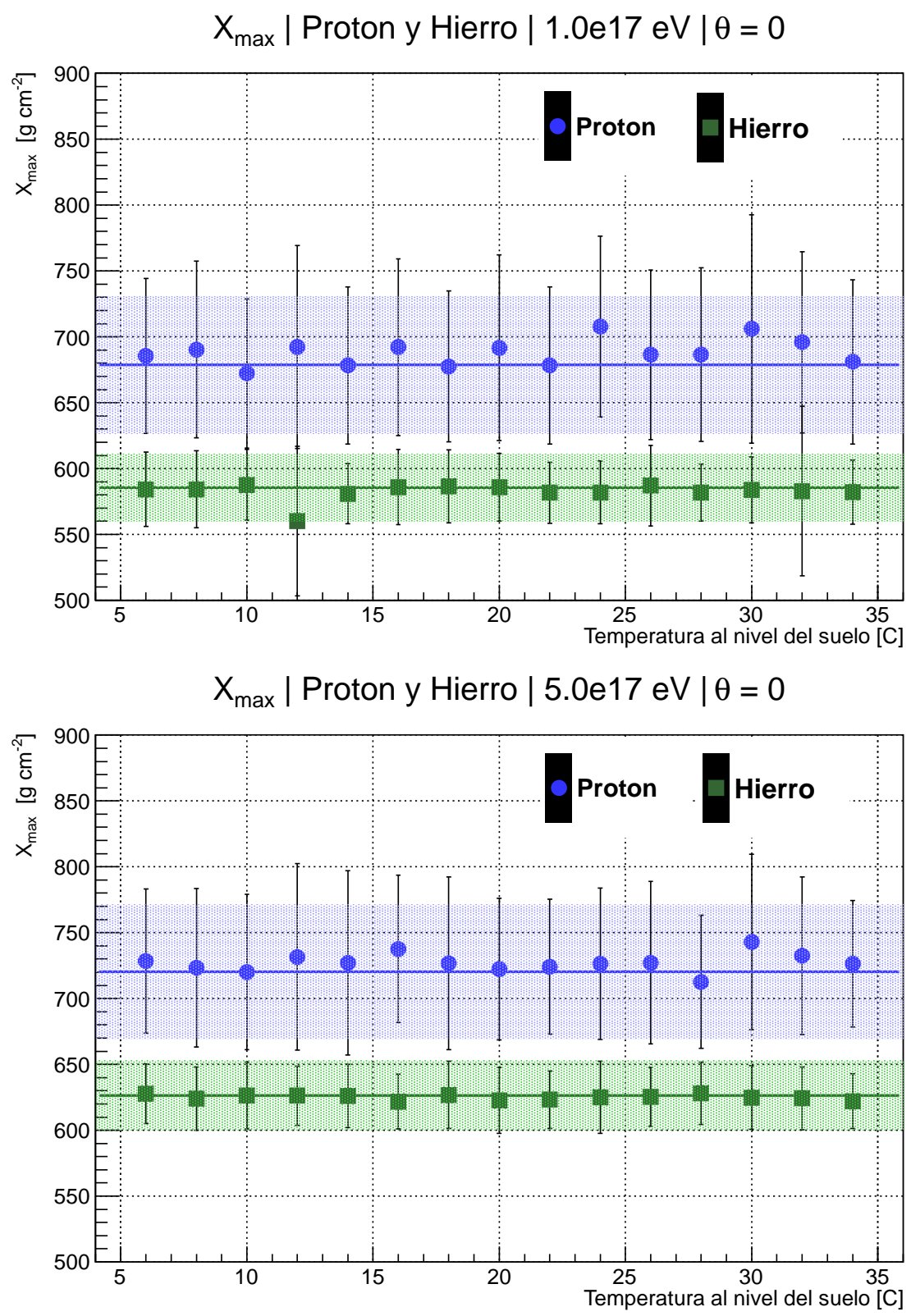

Figura 8.10: Valores promedio de $X_{\max }$ de lluvias originadas por protones y núcleos de hierros de $10^{17} \mathrm{eV}$ y $5 \times 10^{17} \mathrm{eV}$; y desarrolladas en diferentes atmósferas del modelo GAMMA identificadas por el valor su temperatura al nivel del suelo. Las líneas representan el valor promedio de $X_{\max }$ correspondiente al desarrollo de lluvias con iguales primarios y energías pero desarrollada en una atmósfera de Linsley; mientras que las zonas sombreadas demarcan la incerteza asociada al promedio. 

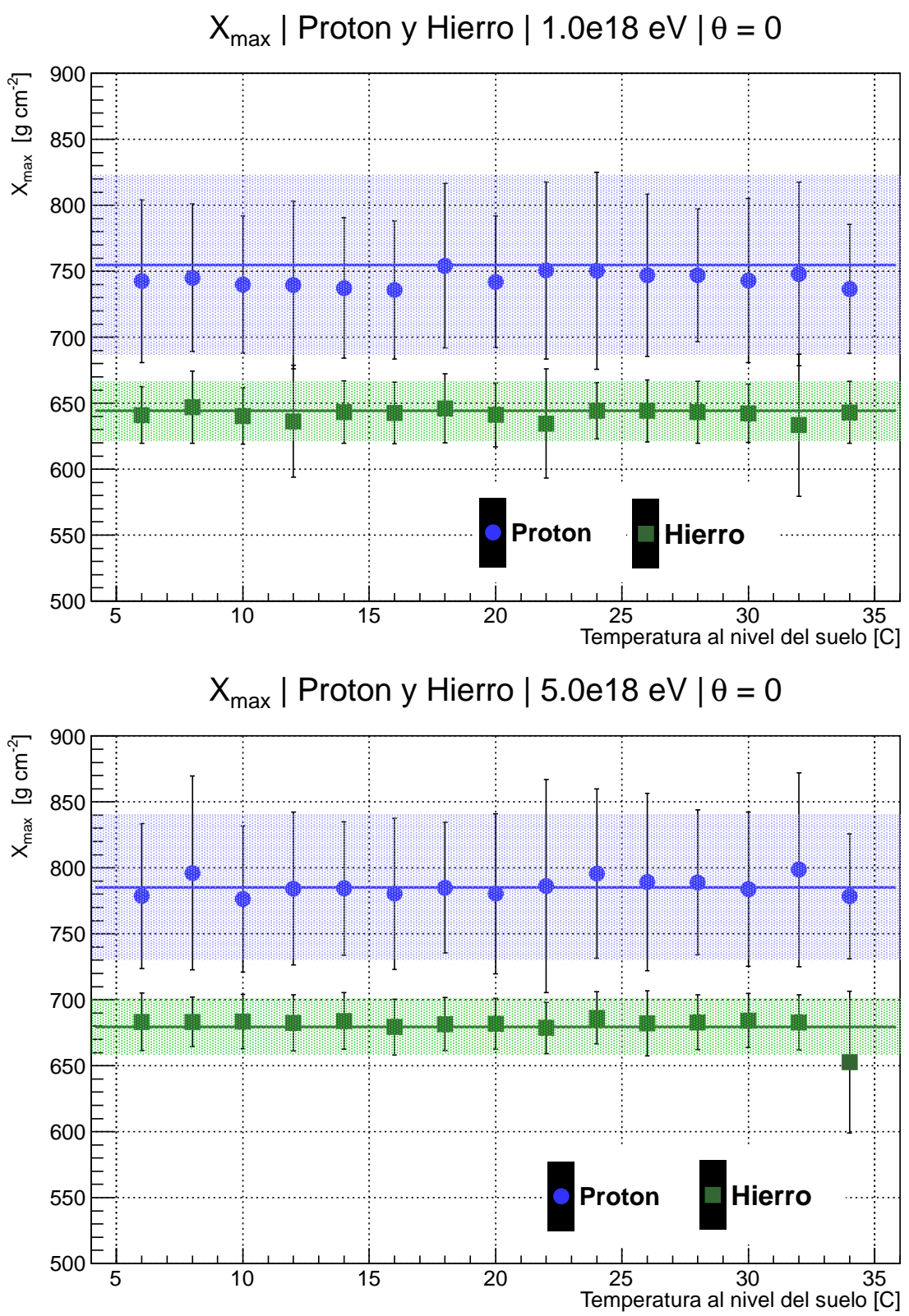

Figura 8.11: Valores promedio de $X_{\max }$ de lluvias originadas por protones y núcleos de hierros de $10^{18} \mathrm{eV}$ y $5 \times 10^{18} \mathrm{eV}$; y desarrolladas en diferentes atmósferas del modelo GAMMA identificadas por el valor su temperatura al nivel del suelo. Las líneas representan el valor promedio de $X_{\max }$ correspondiente al desarrollo de lluvias con iguales primarios y energías pero desarrollada en una atmósfera de Linsley; mientras que las zonas sombreadas demarcan la incerteza asociada al promedio. 


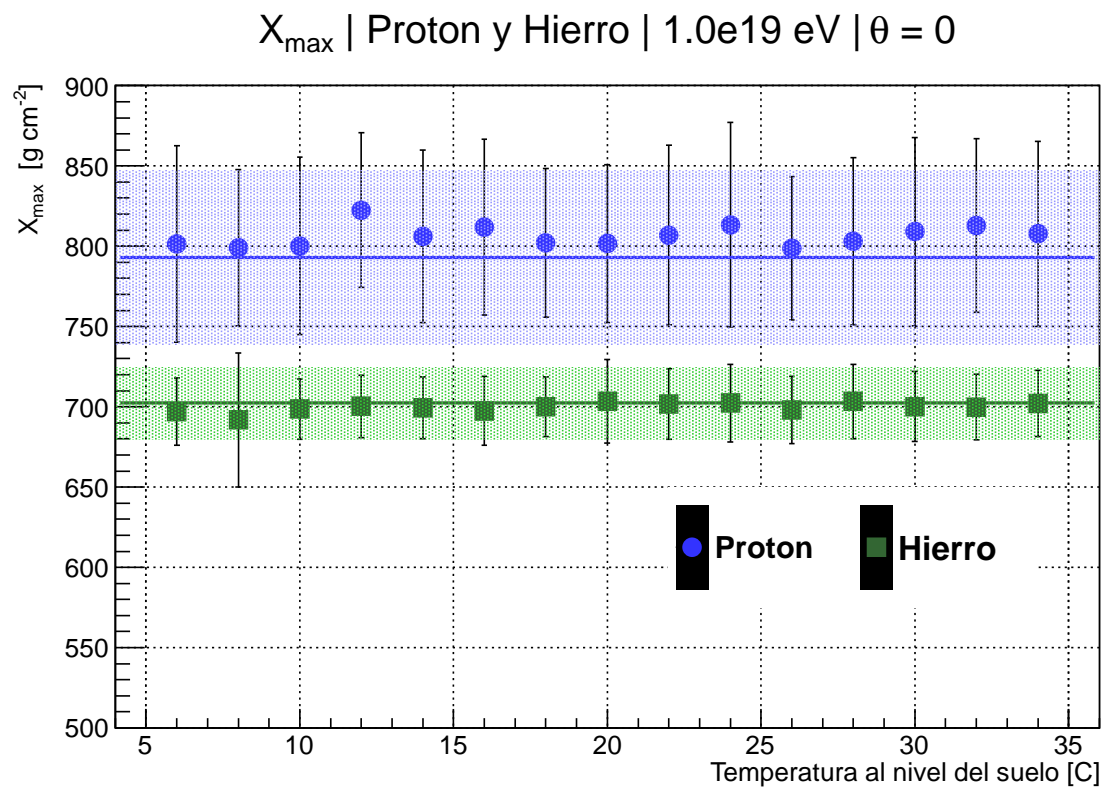

Figura 8.12: Valores promedio de $X_{\max }$ de lluvias originadas por protones y núcleos de hierros de $10^{19} \mathrm{eV}$; y desarrolladas en diferentes atmósferas del modelo GAMMA identificadas por el valor su temperatura al nivel del suelo. Las líneas representan el valor promedio de $X_{\max }$ correspondiente al desarrollo de lluvias con iguales primarios y energías pero desarrollada en una atmósfera de Linsley; mientras que las zonas sombreadas demarcan la incerteza asociada al promedio. 


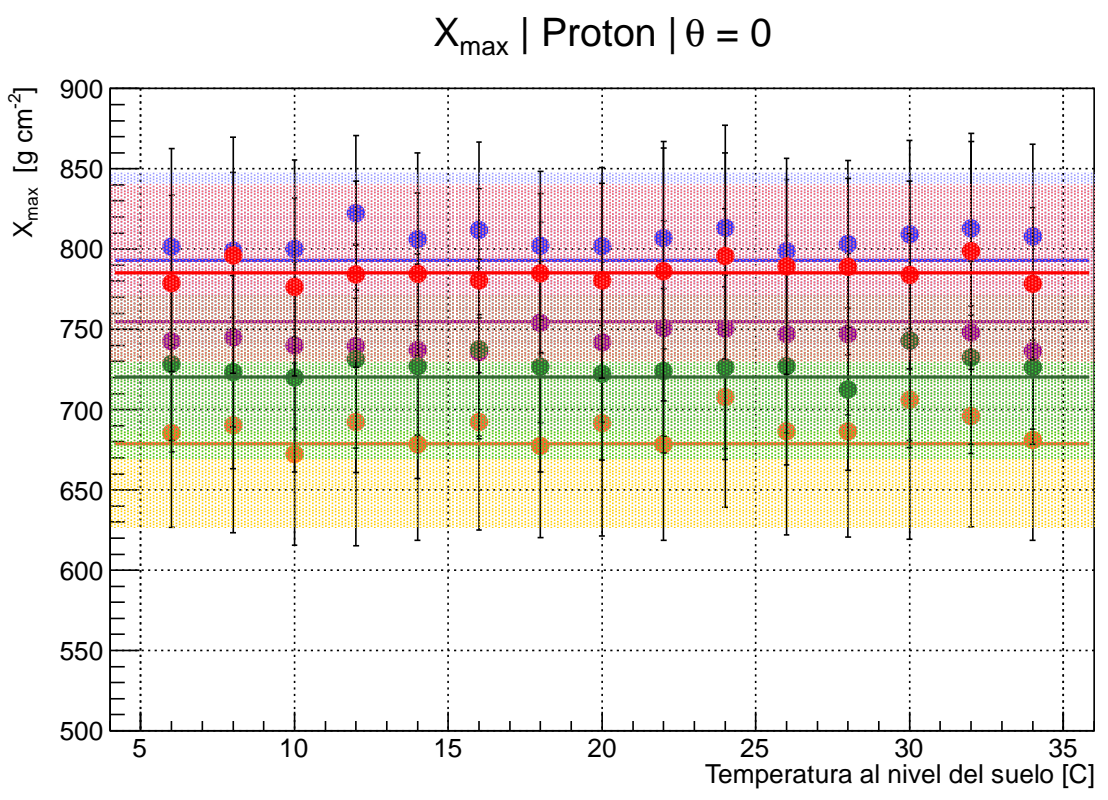

Figura 8.13: Valores promedio de $X_{\max }$ de lluvias originadas por protones de $10^{17} \mathrm{eV}$ (azul), $5 \times 10^{17} \mathrm{eV}$ (rojo), $10^{18} \mathrm{eV}$ (violeta), $5 \times 10^{18} \mathrm{eV}$ (verde), $10^{19}$ eV (naranja); y desarrolladas en diferentes atmósferas del modelo GAMMA identificadas por el valor su temperatura al nivel del suelo. Las lluvias generadas por primarios de mayor energía son más profundas, por lo cual el valor de $X_{\max }$ es mayor. Las líneas representan el valor promedio de $X_{\max }$ correspondiente al desarrollo de lluvias con iguales primarios y energías pero desarrollada en una atmósfera de Linsley; mientras que las zonas sombreadas demarcan la incerteza asociada al promedio. 


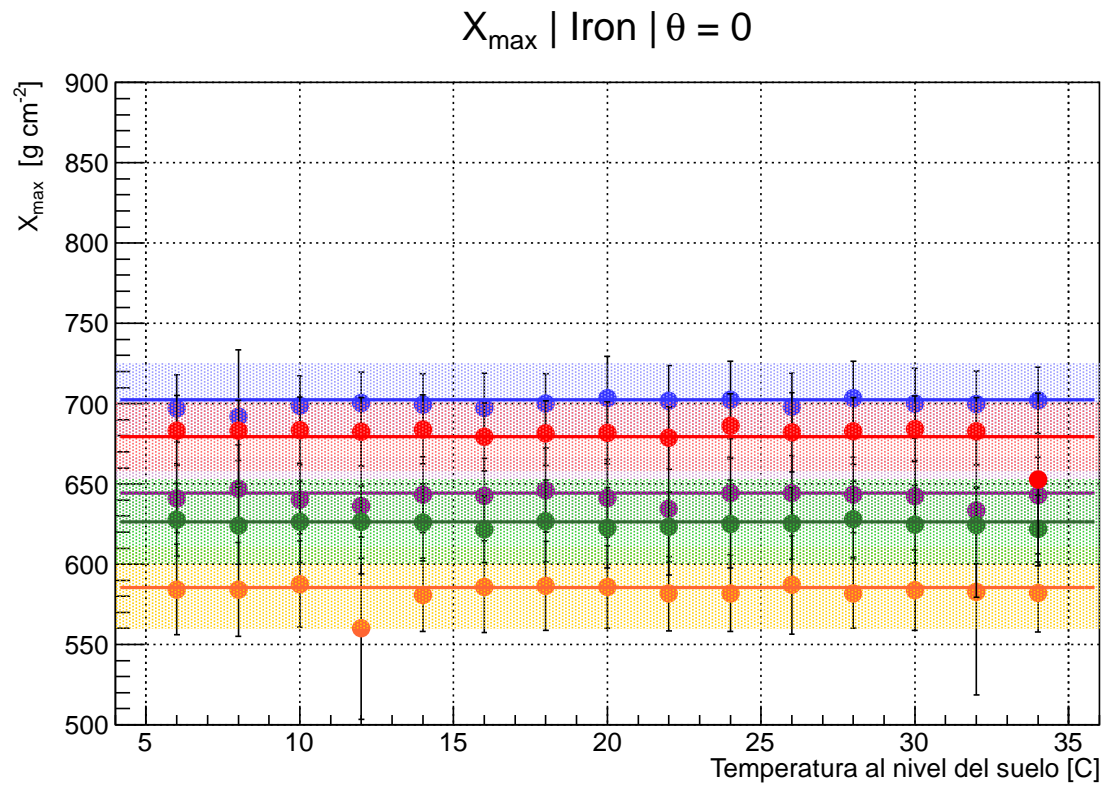

Figura 8.14: Valores promedio de $X_{\max }$ de lluvias originadas por núcleos de hierro de $10^{17} \mathrm{eV}$ (azul), $5 \times 10^{17} \mathrm{eV}$ (rojo), $10^{18} \mathrm{eV}$ (violeta), $5 \times 10^{18} \mathrm{eV}$ (verde), $10^{19}$ eV (naranja); y desarrolladas en diferentes atmósferas del modelo GAMMA identificadas por el valor su temperatura al nivel del suelo. Las lluvias generadas por primarios de mayor energía son más profundas, por lo cual el valor de $X_{\max }$ es mayor. Las líneas representan el valor promedio de $X_{\max }$ correspondiente al desarrollo de lluvias con iguales primarios y energías pero desarrollada en una atmósfera de Linsley; mientras que las zonas sombreadas demarcan la incerteza asociada al promedio. 

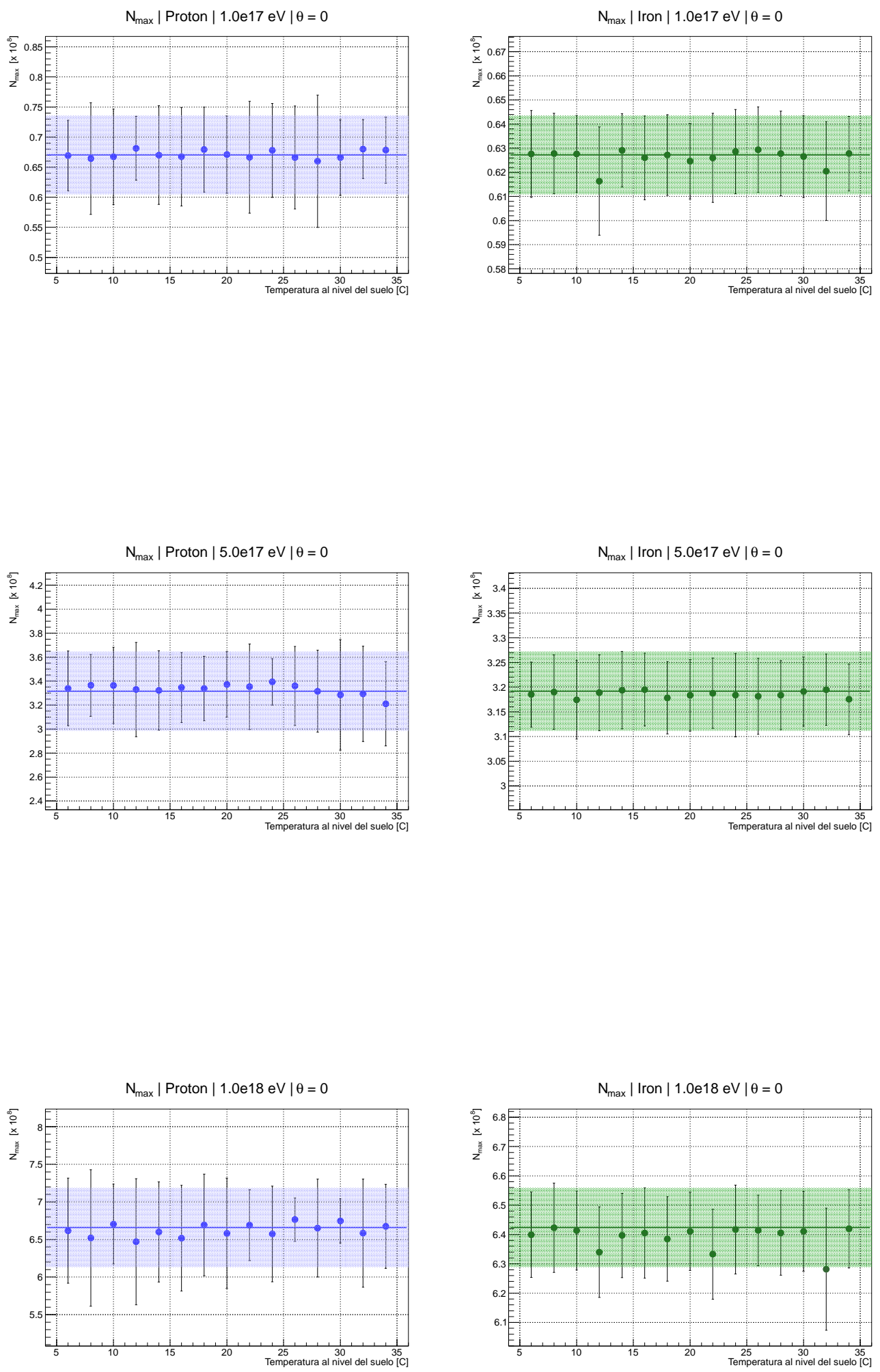

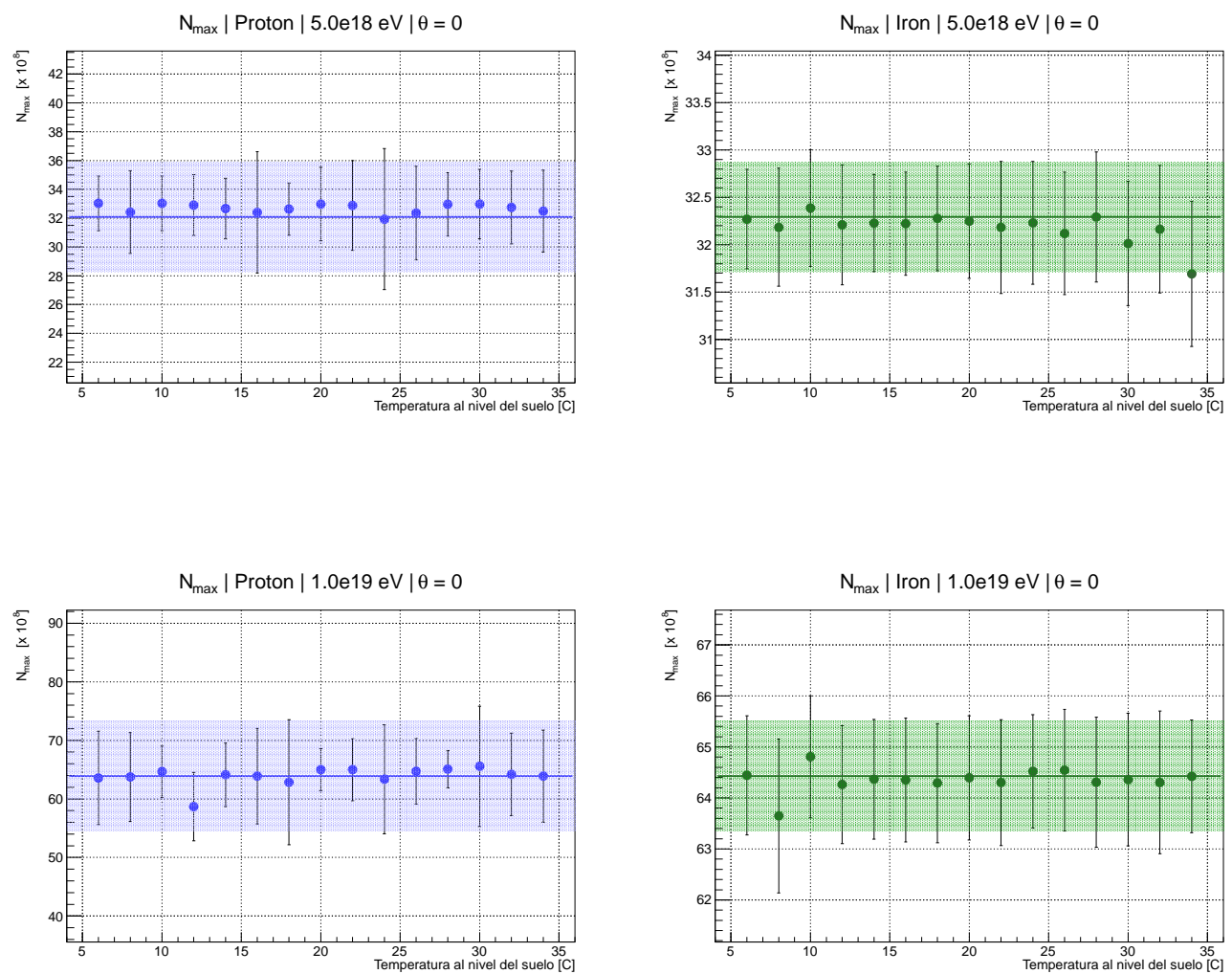

Figura 8.15: Valores promedio de $N_{\max }$ de lluvias originadas por protones $y$ núcleos de hierros de $10^{17} \mathrm{eV}, 5 \times 10^{17} \mathrm{eV}, 10^{18} \mathrm{eV}, 5 \times 10^{18} \mathrm{eV}, 10^{19} \mathrm{eV} ; \mathrm{y}$ desarrolladas en diferentes atmósferas del modelo GAMMA identificadas por el valor su temperatura al nivel del suelo. Las líneas representan el valor promedio de $N_{\max }$ correspondiente al desarrollo de lluvias con iguales primarios y energías pero desarrollada en una atmósfera de Linsley; mientras que las zonas sombreadas demarcan la incerteza asociada al promedio.

\subsubsection{GAMMA y el número de partículas cargadas}

Con el objetivo de estudiar la influencia del uso del modelo atmosférico GAMMA en el desarrollo longitudinal a partir del número de partículas cargadas, se procedió a simular una serie de lluvias en diferentes perfiles atmosféricos derivados del modelo GAMMA. El conjunto de lluvias, también simulado con AIRES tenía la siguiente características:

- Partículas primarias: protones y núcleos de hierro.

- Energía del primario: $10^{19} \mathrm{eV}$. 
- Eje de la lluvia vertical: ángulo cenital $60^{\circ}$.

- Modelo Atmosférico: GAMMA con 15 temperaturas diferentes al nivel del suelo (tomadas cada $2 \mathrm{C}$ entre $6 \mathrm{C}$ y $34 \mathrm{C}$ ) y Linsley.

- Thinning 1.0E-6 Relative

- ThinningWF 0.5

- Injection $115 \mathrm{~km}$

- Ground 1.406 km

Para cada atmósfera se calculó el promedio sobre 100 lluvias, por lo que el conjunto total de lluvias estudiadas corresponde a 3200 lluvias.

Las figuras 8.16 y 8.17 muestran los desarrollos longitudinales del número de partículas cargadas en función de la altitud para los casos de protón y núcleos de hierro. Se observa que esos desarrollos longitudinales son claramente diferenciables para las distintas atmósferas provistas por GAMMA. La escala cromática, de tonos rojizos para bajas temperaturas y azulados para las altas, permite observar que el corrimiento de la posición del máximo es sistemático según aumenta la temperatura que define la atmósfera GAMMA. El mismo punto es evidente tanto para las lluvias inducidas por protones como las inducidas por hierros ${ }^{2}$.

En las gráficas puede observarse la clara diferencia entre la profundidad alcanzada por el desarrollo de las cascadas originadas por protones y las originadas por hierros. Los primeros tienen en promedio un desarrollo máximo alrededor de los $7.51 \mathrm{~km}$ y los segundos en los $8.55 \mathrm{~km}$,f de acuerdo con lo esperado.

Observemos particularmente la representación simultánea de los casos extremos: el promedio de las lluvias inducidas por protones en una atmósfera GAMMA de $34 \mathrm{C}$ y el promedio de lluvias inducidas por hierros en una atmósfera de $6 \mathrm{C}$ (Figura 8.18 arriba). Es evidente que las lluvias poseen un perfil de desarrollo similar y con los máximos cercanos entre sí. Concretamente los valores obtenidos muestran un máximo de desarrollo a los 7.704 $\mathrm{km}$ en el caso de los protones y de $8.282 \mathrm{~km}$ en el caso de los hierros.

El caso opuesto, lluvias originadas por protones desarrolladas en atmósferas GAMMA de baja temperatura y otras originadas por hierros desarrolladas en atmósferas GAMMA de alta temperatura, también fue analizado

\footnotetext{
${ }^{2}$ Permitiéndonos un abuso en el lenguaje usaremos de forma equivalente los términos "hierros", "núcleos de hierro" y "núcleos de átomos de hierro"; cuando en realidad siempre nos estamos refiriendo a los últimos.
} 

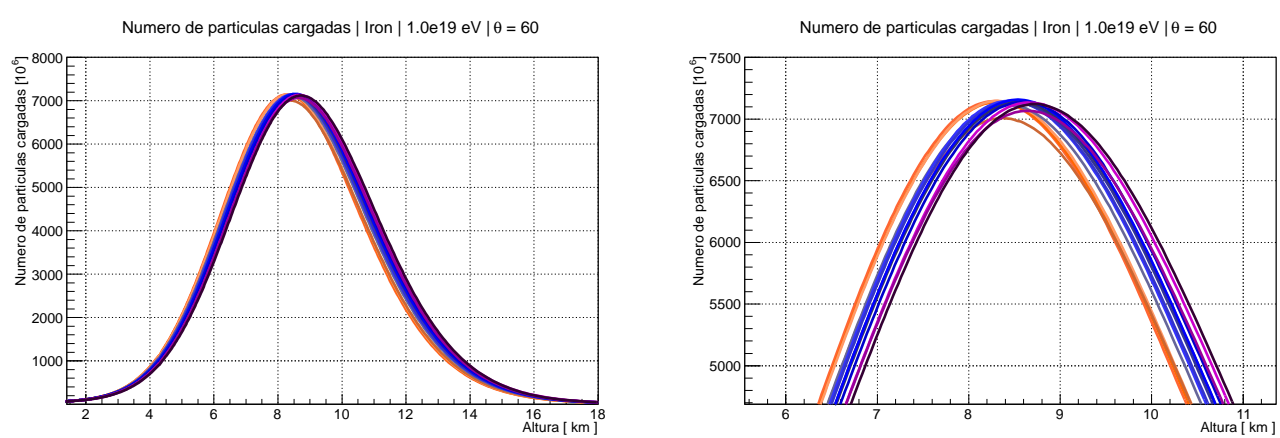

Figura 8.16: Perfiles promedios de desarrollo longitudinal en términos de la altitud correspondientes a lluvias inducidas por núcleos de hierro de $10^{19} \mathrm{eV}$ con un ángulo de incidencia de $60^{\circ}$ (izquierda). Se encuentran graficados 16 perfiles correspondientes a 15 atmósferas GAMMA con temperaturas al nivel del suelo entre 6 C y 34 C y la atmósfera provista por Linsley. La escala cromática del rojizo para las temperaturas bajas hacia los azules para las temperaturas altas muestra el progresivo aumento de la localización del máximo. El zoom (derecha) sobre la zona del máximo permite apreciar mejor este punto.

(Figura 8.18 abajo). Los valores promedio de los máximos logran una separación de $1.25 \mathrm{~km}$ aproximadamente, siendo este valor derivado del máximo en $7.462 \mathrm{~km}$ para el caso de protones y de $8.717 \mathrm{~km}$ en las lluvias inducidas por hierros.

Vale la pena comentar que estos resultados se asemejan a los reportados en [117], obtenidos en base a simulaciones de lluvias inducidas por protones

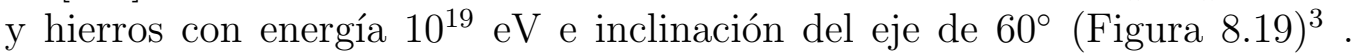
En este caso las lluvias fueron simuladas sobre una atmósfera que representa una noche de invierno y una atmósfera que representa una noche de verano, representadas por los perfiles $\langle 3\rangle$ y $\langle 21\rangle$ respectivamente en la figura. Ambas atmósferas no son producto de modelos sino que corresponden a perfiles medidos en el sitio del Observatorio Auger. Los perfiles de desarrollo resultantes muestran que las lluvias inducidas por hierro sobre la atmósfera invernal alcanzan su máximo aproximadamente a los $8.2 \mathrm{~km}$ de altura, un valor muy similar a los $8.0 \mathrm{~km}$ correspondientes al máximo del perfil en el caso de lluvias desarrolladas sobre la atmósfera estival por protones como primarios.

\footnotetext{
${ }^{3}$ Estas simulaciones fueron generadas por CORSIKA [118] con el modelo de interacción hadrónica QGSJET01 [119]
} 

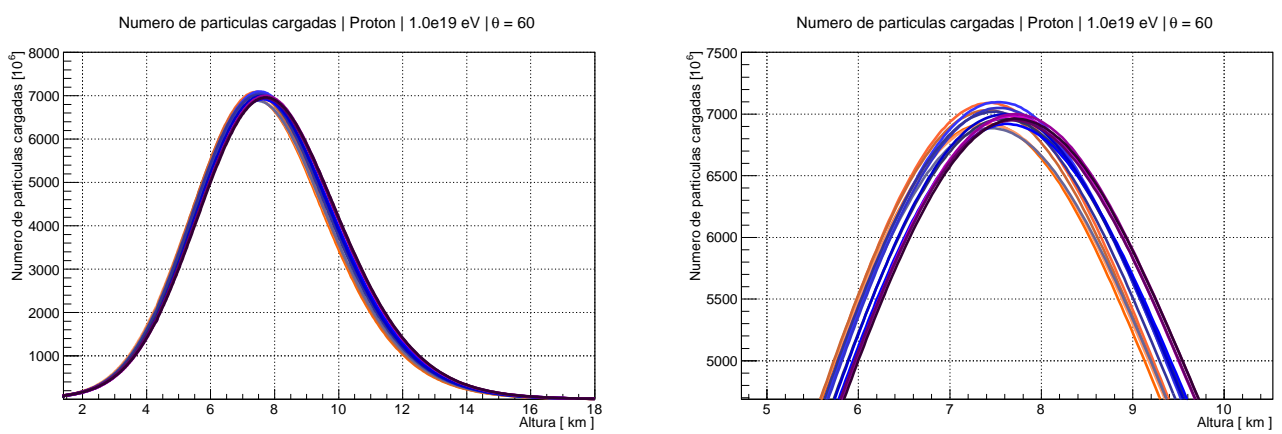

Figura 8.17: Perfiles promedios de desarrollo longitudinal en términos de la altitud correspondientes a lluvias inducidas por protones de $10^{19} \mathrm{eV}$ con un ángulo de incidencia de $60^{\circ}$ (izquierda). Se encuentran graficados 16 perfiles correspondientes a 15 atmósferas GAMMA con temperaturas al nivel del suelo entre 6 C y 34 C y la atmósfera provista por Linsley. Al igual que en el caso del hierro, la escala cromática del rojizo para las temperaturas bajas hacia los azules para las temperaturas altas muestra el progresivo aumento de la localización del máximo, situación mejor apreciada con el zoom sobre la zona del máximo (derecha).

Podemos hacer la comparación con la Figura 8.18 suponiendo que una atmósfera GAMMA de temperatura baja (6 C) representa a una atmósfera de invierno, y una atmósfera GAMMA de temperatura alta $(34 \mathrm{C})$ representa a una atmósfera de verano. En este marco, observamos que ambos planteos evidencian que la combinación protón-temperatura alta (verano) reproduce los desarrollos más similares a los inducidos por hierros (cuando se realizan en atmósferas de temperaturas bajas (invierno)). Sin embargo los resultados en GAMMA muestran máximos mucho más distanciados promoviendo la lectura de perfiles distinguibles.

Cuando las lluvias son simuladas sobre la atmósfera invernal y la estival (Figura 8.19); se observa que las lluvias inducidas por hierro sobre la atmósfera invernal alcanzan su máximo aproximadamente a los $8.2 \mathrm{~km}$ de altura, un valor muy similar a los $8.0 \mathrm{~km}$ correspondientes al máximo del perfil en el caso de lluvias desarrolladas sobre la atmósfera estival por protones como primarios. Esta diferencia es más del doble de la entrada con GAMMA.

Continuando con el estudio del desarrollo longitudinal, se realizó el análisis de la energía depositada. Como planteamos anteriormente, la energía depositada por las partículas en la atmósfera permite la ionización que deriva en la emisión de luz de fluorescencia. Esto implica que si una serie de lluvias originadas por idénticos primarios alcanzan su máximo en lugares diferentes 


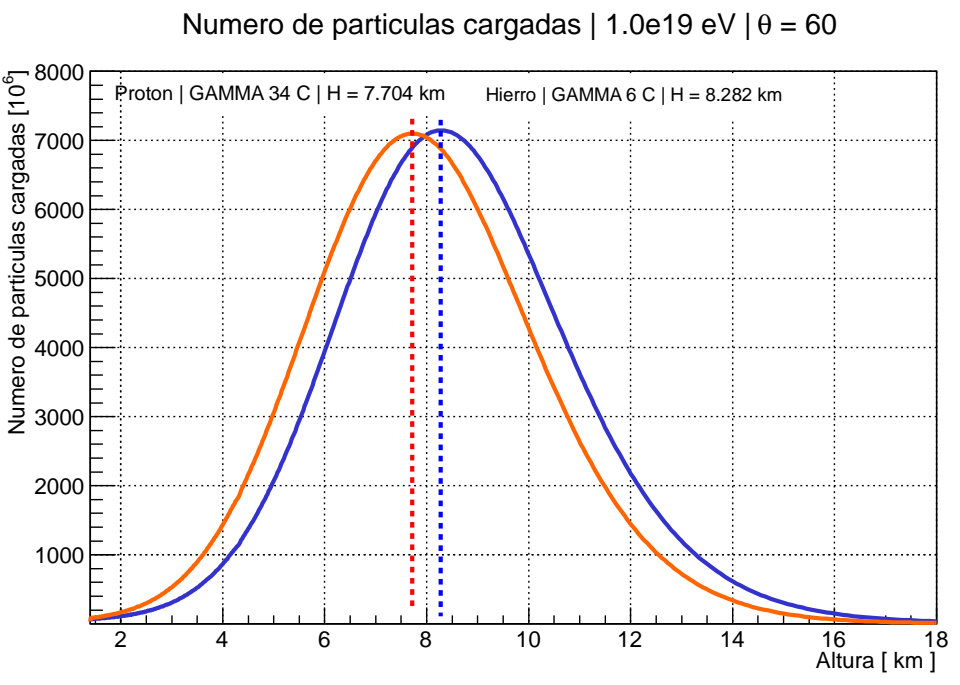

Numero de particulas cargadas $|1.0 \mathrm{e} 19 \mathrm{eV}| \theta=60$

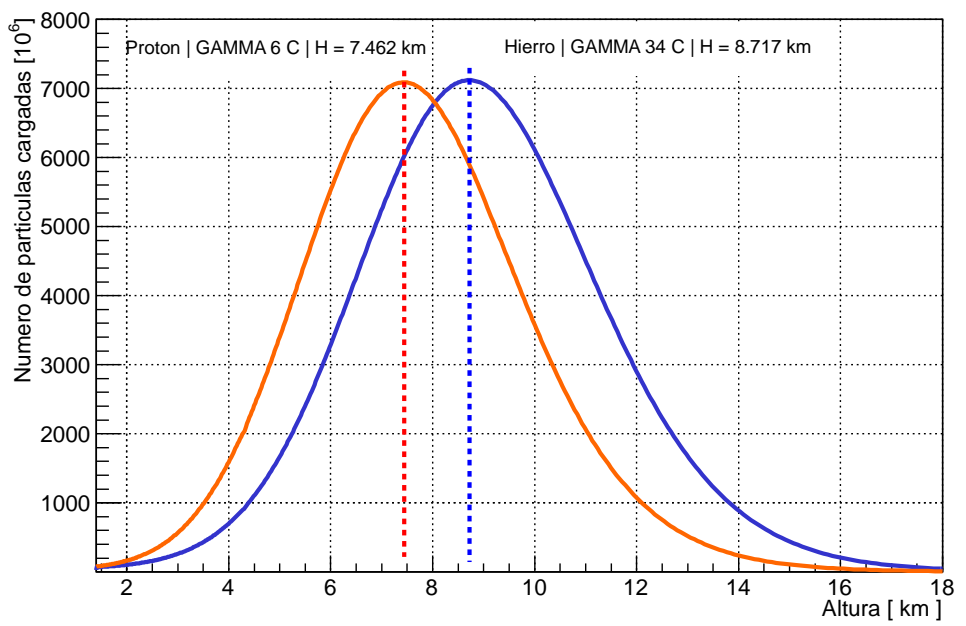

Figura 8.18: Perfiles promedio de desarrollo longitudinal en términos de la altitud correspondientes a 100 lluvias inducidas por núcleos de hierro desarrolladas en una atmósfera GAMMA de $6 C$ e igual número de lluvias originadas por protones y desarrolladas en una atmósfera GAMMA de $34 \mathrm{C}$ (arriba). Igual número de lluvias para los casos de hierros desarrollados en una atmósfera GAMMA de 34 C y protones en GAMMA de 6 C. En todos los casos los primarios tenían una energía de $10^{19} \mathrm{eV}$ y un ángulo de incidencia de $60^{\circ}$. Se encuentran identificadas las alturas a las cuales se percibe el máximo número de partículas cargadas. 


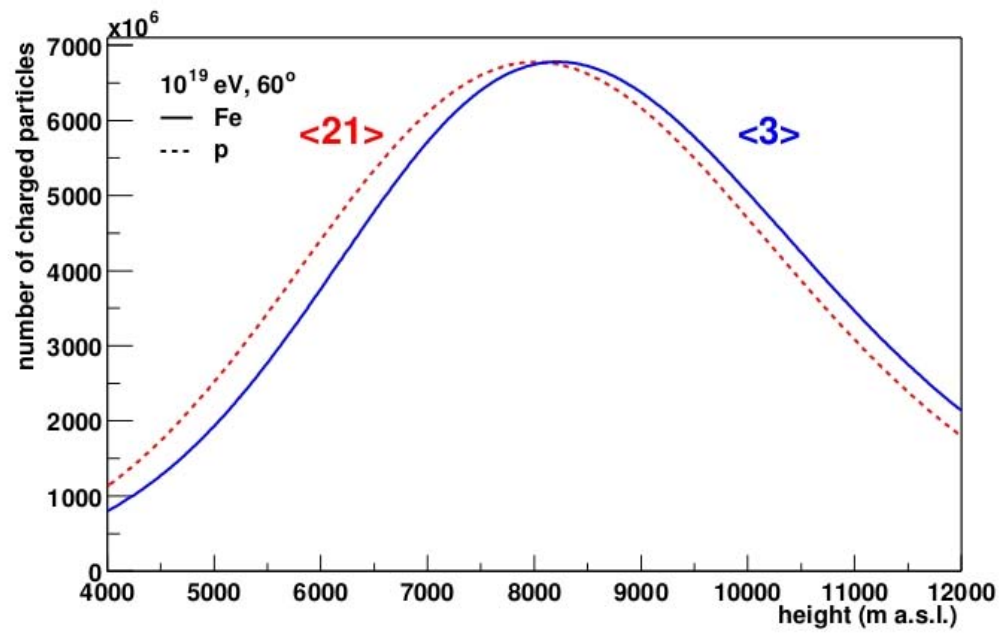

Figura 8.19: Perfiles promedio de número de partículas cargadas para simulaciones de lluvias inducidas por protones desarrolladas en una atmósfera estival ( $\langle 21\rangle)$, y el mismo tipo de perfil para lluvias inducidas por núcleos de hierro desarrollen otra invernal $(\langle 3\rangle)$. Ambas atmósferas provienen de medidas de globos radiosondas en el Observatorio Auger. La energía del primario era de $10^{19}$ eV y el ángulo de incidencia de $60^{\circ}$ [117]. 
de acuerdo a la atmósfera en que se desarrollaron, serán detectadas en forma distinta por los detectores de fluorescencia generando estimaciones erróneos de los observables. Un ejemplo de esta situación puede ser observado a partir de los perfiles promedios correspondientes a la variación de la energia depositada con la altura para el caso de lluvias generadas por hierro en todas las atmósferas GAMMA (Figura 8.20 arriba), y particularmente en forma separada las de 6 C, y 34 C (Figura 8.20 abajo).

Estas figuras muestran que las lluvias alcanzan su máximo desarrollo a menor altura (mayor profundidad atmosférica) en atmósferas correspondientes a temperaturas bajas. Además, como es esperable debido a la relación entre el número de partículas cargadas y la energía depositada por las mismas, en la representación de todas las atmósferas los resultados entre la Figura 8.20 (arriba) y la Figura 8.16 son muy similares. A partir de la Figura 8.20 (abajo) se observa que la diferencia en el corrimiento del lugar de máximo desarrollo resulta del orden de los $435 \mathrm{~m}$ entre las atmósferas extremas de GAMMA con 6 C y GAMMA con 34 C.

Esta expresión del desarrollo longitudinal en términos de la energía depositada puede ser contrastada con resultados ya publicados [120] (Figura 8.21). Para ello debemos considerar las mismas suposiciones anteriores (una atmósfera de invierno es representable por una atmósfera GAMMA de temperatura baja, y una de verano por una de temperatura alta). Comparando las figuras 8.20 (abajo) y 8.21, se observa que en ambos casos el máximo del perfil se alcanza a alturas más bajas para bajas temperaturas (invierno). Sin embargo en los perfiles de desarrollo obtenidos con GAMMA se observa menor corrimiento: en [120] establecen una separación entre máximos de 700 $\mathrm{m}$, resultado casi $270 \mathrm{~m}$ mayor que el encontrado con GAMMA.

A modo de cierre del estudio de la influencia GAMMA en el desarrollo longitudinal recapitulemos las conclusiones arribadas. Los resultados encontrados al utilizar el modelo GAMMA en las simulaciones confirman que cascadas de igual energía y primario poseen un desarrollo longitudinal similar en profundidad atmosférica (dentro de la incerteza) pero que expresados en términos de la altura son diferentes de acuerdo al valor de la temperatura en el suelo. Este punto resulta coincidente en esencia con lo planteado en [117] y [120] cuando se supone que el perfil atmósferico invernal (estival) está bien representado por uno obtenido con el modelo GAMMA con bajas (altas) temperaturas. Sin embargo las diferencias en la ubicación del máximo de desarrollo son perceptibles cuando la representación se realiza en términos de altura. Debemos destacar que esas diferencias resultan mayores cuando son obtenidos mediante el uso de la correspondiente parametrización GAMMA (aproximadamente $580 \mathrm{~m}$ ) que en el caso reportados en [117] (aproximadamente $200 \mathrm{~m}$ ). Esto en principio implicaría que la posición del lugar 

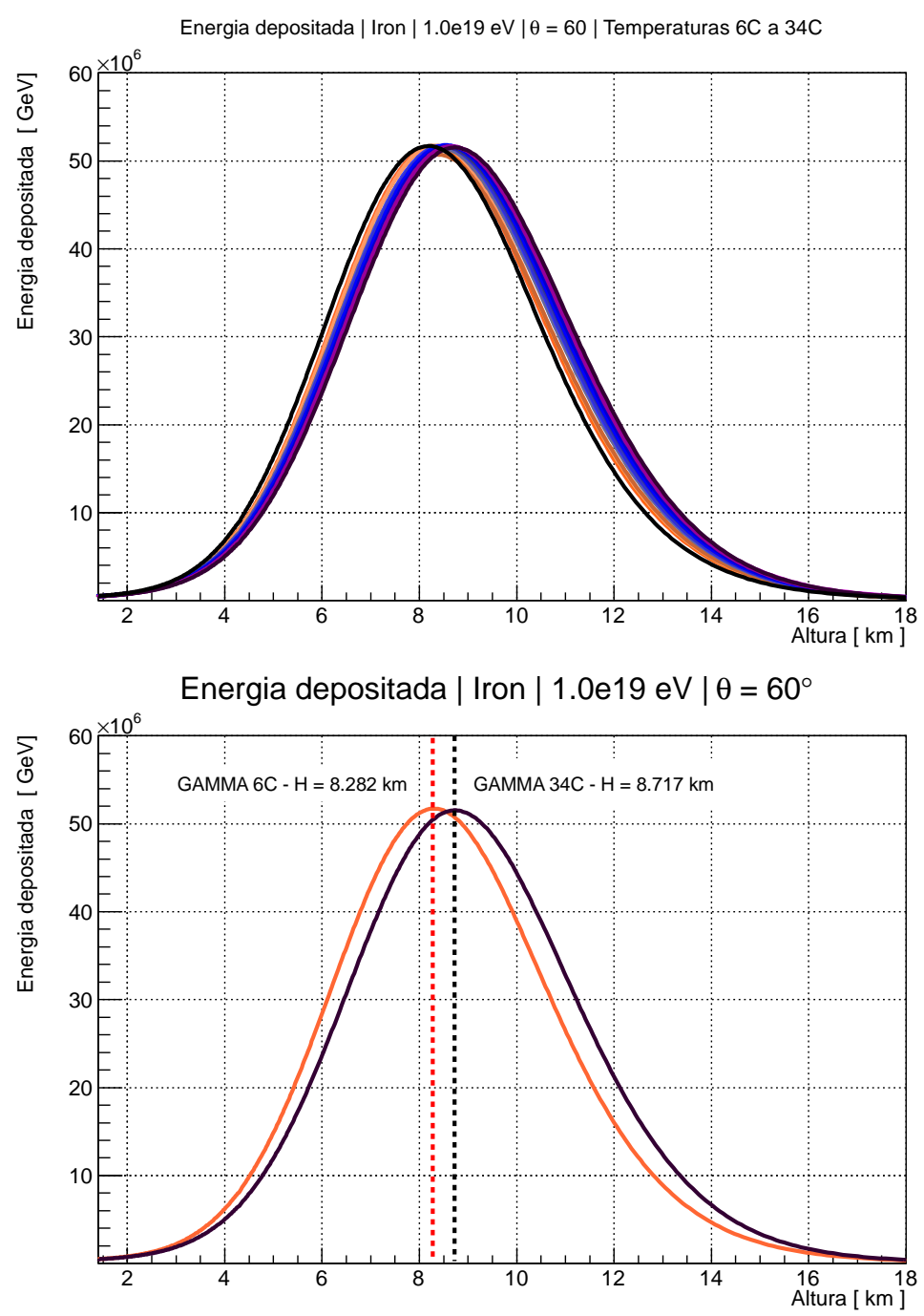

Figura 8.20: Perfiles promedios de desarrollo longitudinal en términos de la altitud correspondientes a lluvias inducidas por hierros de $10^{19} \mathrm{eV}$ con un ángulo de incidencia de $60^{\circ}$ (izquierda). Se graficaron 5 perfiles correspondientes a 4 atmósferas GAMMA con temperaturas al nivel del suelo 6 C, 8 C, 32 C y 34 C; y la atmósfera provista por Linsley. Sin dudas los perfiles son tan similares que resultan prácticamente indistinguibles, incluso con el zoom (derecha) sobre la zona del máximo. 


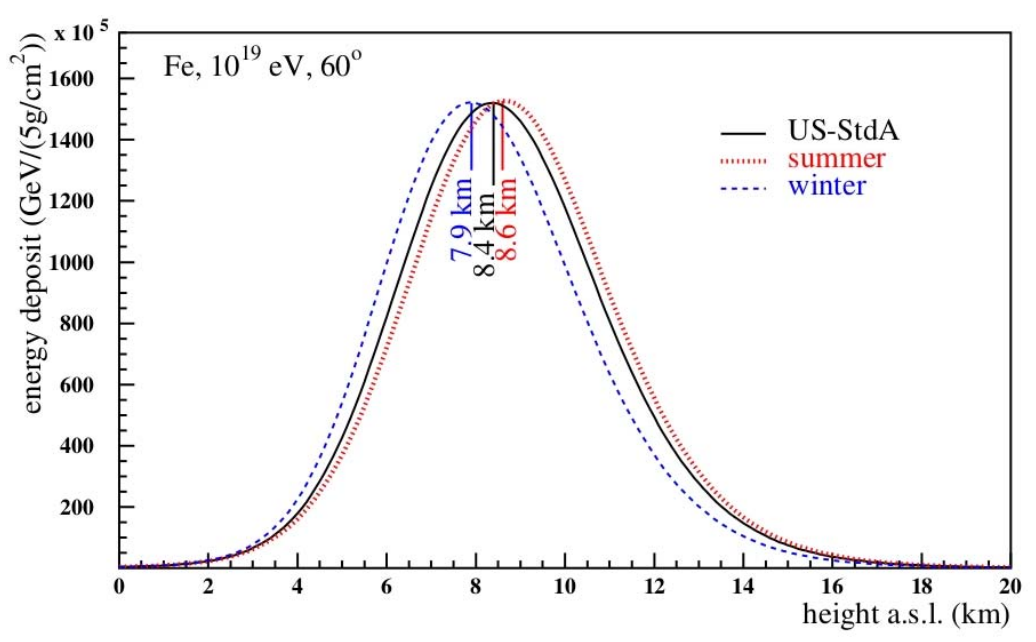

Figura 8.21: Perfiles promedio de energía depositada obtenidos de simulaciones de lluvias inducidas por hierros desarrolladas en una atmósfera estival, invernal y USStdA. Las atmósferas estacionales corresponden a un sitio de latitud medio europea (Stuttgart, Alemania). La energía del primario era de $10^{19}$ eV y el ángulo de incidencia de $60^{\circ}$ [120].

de máximo desarrollo aún sería un parámetro factible en la identificación de la composición de primarios.

Asimismo, el uso del modelo GAMMA entre $6 \mathrm{C}$ y $34 \mathrm{C}$ nos ha permitido observar que lluvias inducidas por el mismo primario (hierro) con la misma energía tendrían localizado su máximo desarrollo comprendido aproximadamente entre los 8280 y $8700 \mathrm{~m}$. Mas allá de los límites resulta relevante que el rango es de unos $435 \mathrm{~m}$, notablemente inferior a los $700 \mathrm{~m}$ observados en [120]. La conclusión arribada mediante el uso de GAMMA es que se verifica que un mismo primario genera perfiles de desarrollo diferentes de acuerdo a la temperatura, lo que implicará diferencias en las medidas de esas lluvias mediante fluorescencia.

No se encuentra en la bibliografía el estudio de la influencia de los modelos MMM o NMMM en los desarrollos longitudinales. Esto impide la comparación entre los perfiles resultantes de lluvias desarrolladas sobre las parametrizaciones de los meses más fríos australes (Junio, Julio, Agosto) de estos modelos con las del modelo GAMMA a bajas temperaturas y las condiciones de invierno retratadas en 8.19 y 8.21 (y algo similar con Diciembre, Enero y Febrero, GAMMA a altas temperaturas y las condiciones del perfil estival). La próxima version de AIRES incluirá también la parametrización de NMMM, lo que permitirá realizar este estudio. 


\section{Capítulo 9}

\section{Resumen, Conclusiones y Perspectivas}

La motivación inicial del desarrollo de un modelo de profundidad atmosférica que represente la atmósfera de Malargüe terminó convirtiendo este tema en el centro de la Tesis. Muchos aspectos contribuyeron a encontrar tareas de estudio que nos mantuvieron continuamente en esta línea de trabajo: implementar variaciones que permitan mejorar las precisiones del modelo GAMMA, el desarrollo paralelo de la actualización del Malargüe Monthly Model al New Malargüe Monthly Model, el proyecto de extender la idea a otras latitudes de manera de plantearlo como un modelo global, la búsqueda de identificar la función más adecuada y la determinación de su impacto en los observables.

Esta Tesis tiene una primera parte monográfica vinculada a la presentación de las características de los rayos cósmicos, sus interacciones con la atmósfera terrestre y su detección en el Observatorio Pierre Auger. En una segunda parte se enfoca el tema de los modelos de profundidad atmosférica, en donde se desarrolla nuestra contribución original: el modelo GAMMA. La construcción de este modelo fue en sus inicios debida a dos motivaciones: (i) la descripción precisa de la atmósfera local, mejorando las predicciones del modelo de Linsley y (ii) la necesidad de considerar las variaciones en los perfiles atmosféricos como función de un parámetro físico (en lugar de lo propuesto por el MMM y NMMM que utilizan un parámetro temporal).

Con estas premisas desarrollamos un modelo que supone que el valor de la temperatura en el suelo es un parámetro adecuado para caracterizar a los perfiles de profundidad atmosférica en cualquier instante de tiempo. El modelo se construyó utilizando una función con una estructura similar a la de Linsley aplicada sobre datos provienentes de mediciones con globos con radiosondas que fueron lanzados en el sitio del Observatorio Auger. 
El modelo GAMMA describe con excelente precisión los perfiles experimentales, resultando los valores medios de los residuos a cualquier altura comprendidos en el rango $2 \mathrm{~g} \mathrm{~cm}^{-2} \pm 4 \mathrm{~g} \mathrm{~cm}^{-2}$, tal como ha sido discutido en el capítulo 6. Por las características del ajuste, en particular lo referente a fijar los valores de la función y su derivada a la máxima altura y dejarlos libre al nivel del suelo, resulta remarcable la precisión lograda por la parametrización GAMMA al nivel del suelo siendo la diferencia con los datos menor a $0.9 \mathrm{~g} \mathrm{~cm}^{-2} \pm 3 \mathrm{~g} \mathrm{~cm}^{-2}$. Todas estas observaciones se pueden identificar e interpretar en los gráficos de residuos de ese capítulo.

Las comparaciones con los otros modelos también resultaron altamente satisfactorias. Las diferencias con los datos experimentales para NMMM y GAMMA son del mismo orden; y ambas claramente superiores a las predicciones del modelo de Linsley. Particularmente queremos destacar las diferencias con el modelo de Linsley - marcadas de diversas maneras en el capítulo 6 porque éste fue pensado como un modelo global que representa una atmósfera promedio en un sitio de latitud media. Más allá de que el modelo GAMMA fue construido sobre datos de un sitio particular, su comparación con Linsley promueve una señal de advertencia al momento de elegir el modelo: dentro de los intereses del estudio, hay que tener un cuidado especial cuando se quiere reproducir los perfiles atmosféricos mediante el uso del modelo de Linsley, especialmente si se quieren representar sitios con temperaturas altas al nivel del suelo. Todas estas comparaciones también resultaron en la validación de la temperatura al nivel del suelo como un buen parámetro para caracterizar los perfiles de profundidad atmosférica.

Los resultados promisorios en la construcción del modelo permiten ampliar el rango de validez del modelo GAMMA de los originales $278 \mathrm{~K}$ - $298 \mathrm{~K}$ hasta 278 K - 304 K manteniendo las incertezas originales. Suponemos que los problemas que motivaron no poder extender el límite del modelo por debajo de los $278 \mathrm{~K}$ se deberían a la cercanía con el punto de congelamiento del agua, y la baja estadística de datos en esa zona no nos permitió explorarlo mejor.

Además se corrobora la precisión alcanzada por el modelo al utilizar una base de datos independiente, también obtenida de globos lanzados en el Observatorio Auger, para contrastar los perfiles medidos con las predicciones de GAMMA. El resultado muestra que las incertezas son prácticamente idénticas a las obtenidas en la construcción del modelo.

En el capítulo 7 fueron desarrollados diversos estudios adicionales vinculados a GAMMA. De ellos, el que tal vez implique más proyección en las mejoras intrínsecas de GAMMA sea el vinculado a suponer la atmósfera como capas con temperatura variable. Este modelo que identificamos como GAMMA-NI lleva los valores medios de los residuos por debajo de $2 \mathrm{~g} \mathrm{~cm}^{-2}$ 
y confirma algo que se vislumbra en el modelo original: los valores medios de los residuos y sus incertezas disminuyen de acuerdo la atmósfera posee un valor de temperatura mayor al nivel del suelo. Incluso fue confirmado que el ajuste de los datos de la USStdA mediante este tipo de estructuras funcionales de capas resulta más preciso que el propio ajuste de Linsley.

El segundo de esos estudios es el vinculado a parametrizar en forma polinómica los coeficientes $c_{l}$ que se obtienen de los ajustes en el desarrollo del modelo GAMMA. Concluimos que ajustar los coeficientes con un polinomio de grado 3 es sin dudas una mejora en el modelo GAMMA ya que las predicciones resultan ser más precisas. Sin embargo como las diferencias no son tan significantes y la estructura de los residuos no cambia, consideramos preferibles mantener la simpleza del ajuste lineal de los coeficientes $c_{l}$.

Algo similar nos permite concluir el uso de un ajuste con mayor cantidad de grados libertad descrito en la sección 7.3. Los resultados obtenidos coinciden con los de GAMMA original dentro de un $1 \mathrm{~g} \mathrm{~cm}^{-2}$, valores que hemos encontrado satisfactorios.

En definitiva GAMMA resulta ser sin dudas un modelo preciso en la descripción de perfiles de profundidad atmosférica en el sitio del Observatorio Auger. El paso siguiente, con mucha proyección para un modelo global, fue testear si la idea madre de considerar el valor de la temperatura al nivel del suelo era aplicable a otros sitios. Los lugares escogidos son Dodge City en los Estados Unidos y la Base Halley en el Polo Sur. La elección de ambos sitios está ligada a la locación de experimentos de rayos cósmicos: en el caso de Dodge City por su cercanía a Lammar (al momento del estudio era un posible emplazamiento para la instalación de un Observatorio de rayos cósmicos en el hemisferio Norte) y en el caso de Halley por ser el Polo sede de varios experimentos, IceCube por ejemplo. Este estudio, basado también en perfiles obtenidos por globos atmosféricos, confirma la estrategia de GAMMA de utilizar la temperatura al nivel del suelo como parámetro determinante. Además de las conclusiones ya vertidas en la sección 7.4, no queremos dejar de mencionar que nuevamente los residuos disminuyen para temperaturas mayores (caso Dodge City) y es posible obtener una parametrización adecuada con temperatura al nivel del suelo por debajo de los $0 \mathrm{C}$ (caso Base Halley).

La influencia del modelo GAMMA en los parámetros observables de las cascadas de partículas fue contada en el capítulo 8. Todos los estudios fueron realizados en base a simulaciones producidas mediante el software AIRES, con el que se generó una librería de lluvias que incluía distintos tipo de partícula primaria, diferentes energías, ángulos de inclinación y atmósferas GAMMA.

Los resultados en la implicancia sobre el desarrollo lateral utilizando la función de distribución lateral tipo NKG usual en los análisis del Observatorio 
Auger, muestran que el modelo de Linsley sobreestima el valor de $\mathrm{S}_{1000}$ que recordemos es el parámetro que permite calcular la energía mediante arreglos de superficie. Esto implicaría que la elección del perfil de profundidad atmosférica en la reconstrucción de lluvias impactaría en el observable energía calculado. Sin embargo de estos resultados no se evidencia una tendencia que identifique la variación de $\mathrm{S}_{1000}$ como función del parámetro temperatura del modelo GAMMA. Es una nueva puerta para la continuación del análisis.

La estructura funcional de la función LDF, determinada básicamente por los exponentes $\gamma$ y $\beta$ permite apreciar dos puntos interesantes: (i) es razonable considerar $\gamma$ nulo en los ajustes sobre datos (que son ajustes sobre pocos puntos) más allá de que esto coincide con la teoría que asume una cascada puramente electromagnética y (ii) los desarrollos en atmósferas GAMMA son coincidentes en su forma, mostrando ser siempre mayores o menores después del valor $R=1000 \mathrm{~m}$.

El estudio de la influencia de los modelos atmosféricos en el desarrollo longitudinal permite verificar que las cascadas poseen un desarrollo muy similar para un dado tipo de primario, energía y ángulo de incidencia. Esto implica por ejemplo que el valor del observable $\mathrm{X}_{\max }$ sea independiente del modelo atmosférico dentro de las incertezas lluvia a lluvia. Sin embargo en la observación de los perfiles de desarrollo longitudinal en términos de la altura, tarea propia de los detectores de fluorescencia, se identifican que esos valores de $\mathrm{X}_{\max }$ se correlacionan con diferentes alturas de acuerdo al modelo atmosférico GAMMA. Concretamente se constata que las lluvias desarrolladas en atmósferas GAMMA con mayor temperatura al nivel del suelo poseen su máximo desarrollo a mayor altura. Asimismo se observa que estas diferencias son suficientes para distinguir desarrollos longitudinales de lluvias producidas por protones o hierros, manteniendo a $\mathrm{X}_{\max }$ como un recomendable parámetro en la discriminación de composición. Otro de los resultados obtenidos permite arribar a conclusiones referidas a lluvias inducidas por un mismo primario de igual energía y ángulo de incidencia. Se advierte que las diferencias en el desarrollo longitudinal expresado en términos de altura, debidas a la simulación sobre diferentes atmósferas provistas por el modelo GAMMA, podrían inducir a errores en la reconstrucción de eventos medidos mediante detectores de fluorescencia.

A la luz de lo descrito en esta Tesis es evidente que el modelo GAMMA resulta muy preciso en la descripción de los perfiles de profundidad atmosférica. Además, a partir de los diferentes perfiles obtenidos por el modelo GAMMA se perciben influencias en el desarrollo de las cascadas como la sobreestimación en el valor de $\mathrm{S}_{1000}$ y las diferencias en altura del desarrollo longitudinal.

Varias de las perspectivas de trabajo futuras se vislumbran de lo anterior. En términos de desarrollo del modelo GAMMA el principal objetivo 
será intentar darle características de modelo global. En esa dirección se cree necesesario un estudio que involucre no sólo al valor de la temperatura al nivel del suelo sino explorar la dependencia con otros parámetros físicos (concretamente la combinación con la presión al nivel del suelo es un promisorio punto de partida) y otros como la latitud. En este sentido, es de destacar un trabajo recientemente publicado por la Colaboración Pierre Auger, organizado por el grupo de monitoreo atmosférico, en donde se analiza en detalle la conveniencia del uso del Global Data Assimilation System (GDAS), basado en datos medidos desde satélites meteorológicos geoestacionarios [122]. Este sistema, claramente aplicable también para otros experimentos debido a la amplia cobertura del globo terrestre, permite conocer los datos de la atmósfera en el momento del evento gracias a parametrizaciones realizadas sobre medidas meteorológicas y predicciones numéricas del clima. En el caso particular del perfil de densidades, este sistema retorna, para un dado instante, un pequeño conjunto de datos (sensiblemente menos extenso que los obtenidos al lanzar un globo meteorológico en el sitio). El perfil completo de densidades a alturas arbitrarias puede ser obtenido utilizando estos datos para ajustar un modelo como GAMMA. El hecho de que el sistema GDAS pueda ser utilizado en todo el globo lo torna una herramienta potencialmente poderosa para estudiar la generalización de GAMMA, tal como se expresó anteriormente.

Continuar en establecer la influencia en los observables es el otro gran punto de ataque. Con ese objetivo es que se está completando la ampliación de la librería de simulaciones a 160000 lluvias con diferentes combinaciones de tipo de partícula, energía, ángulo de incidencia y modelo atmosférico. Esto permitirá por ejemplo, a partir de analizar sobre diferentes valores de sec $\theta$ ahondar en la búsqueda de establecer un comportamiento para la variación de $\mathrm{S}_{1000}$ con el valor de temperatura en el suelo. $\mathrm{Y}$ sin lugar a dudas, la influencia en el desarrollo longitudinal ha resultado un punto muy intrigante que a partir de la ampliación de la librería podrá ser más exhaustivamente explorado.

Dentro de las perspectivas no queremos dejar de mencionar las relacionadas con las expectativas propias referidas al impacto de este trabajo. Muchos de estos resultados ya han sido presentados en reuniones internas del Observatorio Auger y otros ámbitos, generando productivas críticas y sugiriendo puntos de ajuste y nuevos estudios (muchos de ellos ya realizados y presentados en esta Tesis). La aceptación del modelo es un hecho del que queda esperar extender el uso. Creemos que ayudará significativamente la actualización del software AIRES con el modelo GAMMA disponible. Esto permitirá integrar a la comunidad científica al estudio y análisis de la influencia de los modelos de profundidad atmosférica en el desarrollo de cascadas. 
Appendices 



\section{Apéndice A}

\section{Obtención de los parámetros de $X_{G A M M A}$}

La función $X_{\text {GAMMA }}$ de 7 capas definida por el GAMMA Model en el capítulo 6 queda determiando por los parámetros $a_{l}, b_{l}$ y $c_{l}(l=1, \ldots, 5)$. Los valores para estos parámetros surgen de las condiciones planteadas: continuidad para la función y su derivada y empalme con los valores de la función de Linsley en las capas 6 y 7 , es decir aquellas en las que no había datos experimentales.

Los parámetros pueden ser calculados de la siguiente manera. Es conveniente recordar primero que los parámetros $c_{l}$ cumplen

$$
c_{l}\left(T_{G R O U N D}\right)=M_{l} T_{G R O U N D}+N_{l} \quad(l=1, . ., 5)
$$

donde los valores $M_{l}$ y $N_{l}$ provienen de la Tabla 6.3.

Los valores de los parámetros $a_{l}$ y $b_{l}$ surgen de la imposición de las condiciones planteadas anteriormente. Éstas pueden ser expresadas como

$$
\begin{aligned}
& b_{5}=0,00381472 c_{5} e^{h_{5} / c_{5}} \mathrm{~g} \mathrm{~cm}^{-2} \\
& b_{4}=b_{5} \frac{c_{4}}{c_{5}} e^{-h_{4} / c_{5}} e^{h_{4} / c_{4}} \quad \mathrm{~g} \mathrm{~cm}^{-2} \\
& b_{3}=b_{4} \frac{c_{3}}{c_{4}} e^{-h_{3} / c_{4}} e^{h_{3} / c_{3}} \quad \mathrm{~g} \mathrm{~cm}^{-2} \\
& b_{2}=b_{3} \frac{c_{2}}{c_{3}} e^{-h_{2} / c_{3}} e^{h_{2} / c_{2}} \quad \mathrm{~g} \mathrm{~cm}^{-2} \\
& b_{1}=b_{2} \frac{c_{1}}{c_{2}} e^{-h_{1} / c_{2}} e^{h_{1} / c_{1}} \quad \mathrm{~g} \mathrm{~cm}^{-2} \\
& a_{5}=3,0396-b_{5} e^{-h_{5} / c_{5}} \quad \mathrm{~g} \mathrm{~cm}^{-2} \\
& a_{4}=a_{5}+b_{5} e^{-h_{4} / c_{5}}-b_{4} e^{-h_{4} / c_{4}} \mathrm{~g} \mathrm{~cm}^{-2} \\
& a_{3}=a_{4}+b_{4} e^{-h_{3} / c_{4}}-b_{3} e^{-h_{3} / c_{3}} \mathrm{~g} \mathrm{~cm}^{-2} \\
& a_{2}=a_{3}+b_{3} e^{-h_{2} / c_{3}}-b_{2} e^{-h_{2} / c_{2}} \mathrm{~g} \mathrm{~cm}^{-2} \\
& a_{1}=a_{2}+b_{2} e^{-h_{1} / c_{2}}-b_{1} e^{-h_{1} / c_{1}} \mathrm{~g} \mathrm{~cm}^{-2}
\end{aligned}
$$


Como fue aclarado previamente, los valores de $a_{l}, b_{l}$ y $c_{l}(l=6,7)$ son derivados del Modelo de Linsley Model de 5 capas:

$$
\begin{array}{rccc}
a_{6} & = & 0 & \mathrm{~g} \mathrm{~cm}^{-2} \\
b_{6} & = & 540.1778 & \mathrm{~g} \mathrm{~cm}^{-2} \\
c_{6} & = & 7721.7016 & \mathrm{~m} \\
& & \\
a_{7} & = & 0.01128292 & \mathrm{~g} \mathrm{~cm}^{-2} \\
b_{7} & = & 1.0 & \mathrm{~g} \mathrm{~cm}^{-2} \\
c_{7} & = & 10000000 & \mathrm{~m}
\end{array}
$$

En resumen, la secuencia para calcular la función característica $X_{G A M M A}$ correspondiente al perfil de profundidad atmosférica propio de $T_{G R O U N D}$,

$$
X_{\text {GAMMA }}=\left\{\begin{array}{cc}
a_{l}+b_{l} e^{-\frac{h}{c_{l}}} & h_{l-1} \leq h<h_{l} \quad(l=1, \ldots, 6) \\
a_{7}-b_{7} \frac{h}{c_{7}} & h_{6} \leq h<h_{7} \\
0 & h \geq h_{7}
\end{array}\right.
$$

comienza obteniendo los $c_{l}$ a partir de la Temperatura en suelo $T_{G R O U N D}$ con la expresión [8.1] y los valores de Tabla 6.3, y a partir de ellos obtener los $a_{l}$ y $b_{l}$ mediante[8.2] y [8.3] . 


\section{Apéndice B}

\section{Geometría de las lluvias para el cálculo de la LDF}

Este apéndice busca esclarecer cómo es la geometría detrás del cálculo de la contribución de cada partícula que llega al suelo a la Lateral Distribution Function.

La idea central es que a partir de las coordenadas del punto $P=\left(x_{i}, y_{i}\right)$ de cada partícula en el suelo, y utilizando también los parámetros geométricos de la lluvia - el ángulo cenital $\theta$ y el azimutal $\phi$ - poder calcular el parámetro $r_{0}$ que corresponde a la mínima distancia entre $P$ y el eje de la lluvia.

A partir de la figura B.1, son triviales las siguientes ecuaciones

$$
\begin{gathered}
r^{2}=x_{i}^{2}+y_{i}^{2} \\
\varphi=\operatorname{atan}\left(\frac{y_{i}}{x_{i}}\right)
\end{gathered}
$$

Considerando la figura B.1 abajo-izq, también resulta natural establecer

$$
\begin{aligned}
\operatorname{sen}(\phi-\varphi) & =\frac{M}{r} \\
\cos (\phi-\varphi) & =\frac{L}{r}
\end{aligned}
$$

y de esta última expresión deducimos

$$
L=r \cos (\phi-\varphi)
$$



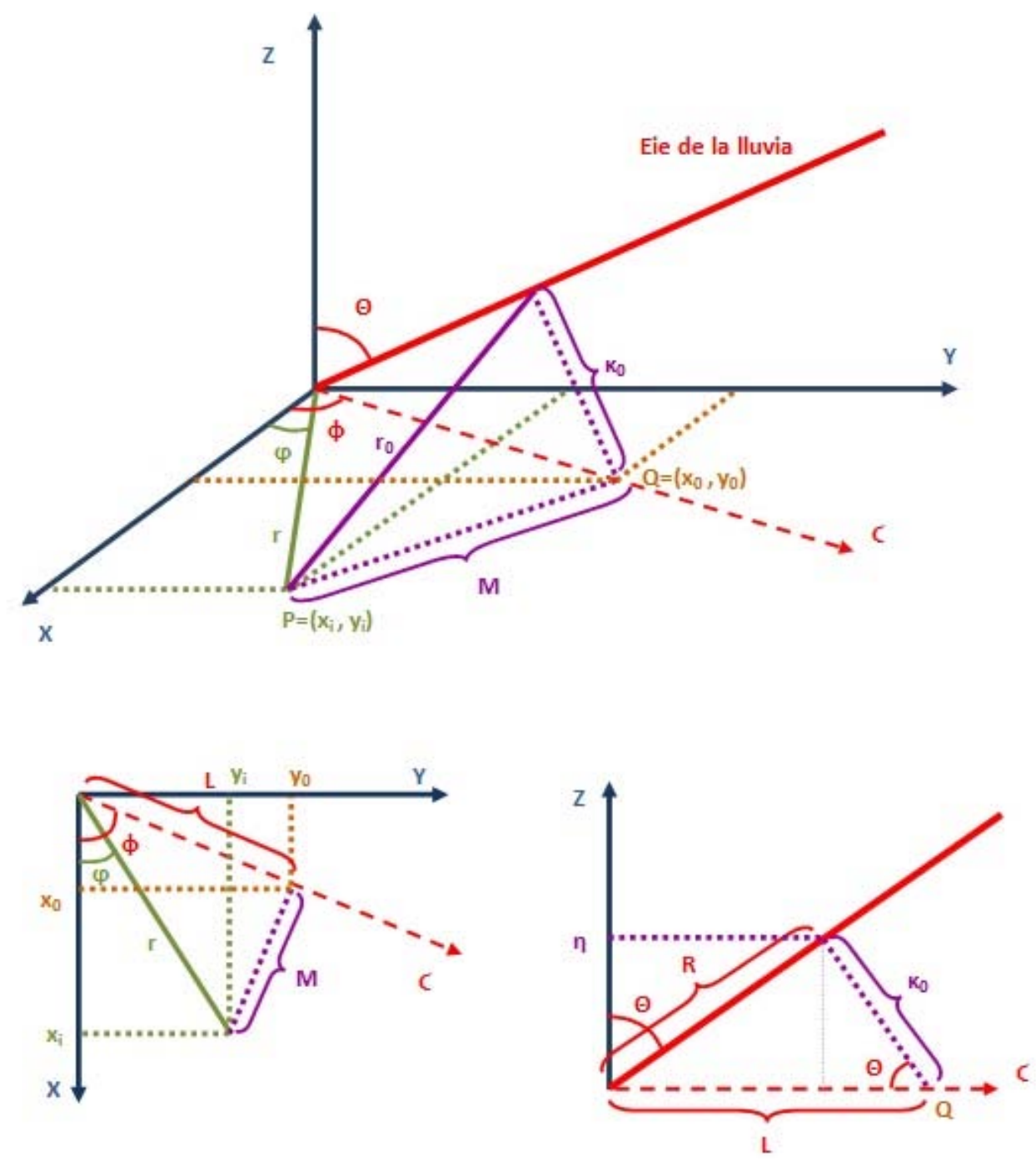

Figura B.1: Representación de la Geometría de la lluvia.

Es inmediato deducir entonces las coordenadas del punto $Q=\left(x_{0}, y_{0}\right)$ que resultan ser

$$
\begin{aligned}
& x_{0}=L \cos (\phi)=r \cos (\phi-\varphi) \cos (\phi) \\
& y_{0}=L \operatorname{sen}(\phi)=r \cos (\phi-\varphi) \operatorname{sen}(\phi)
\end{aligned}
$$

Por otra parte, de la figura B.1 abajo-derecha, tenemos que

$$
\eta=R \cos (\theta)=\kappa_{0} \operatorname{sen}(\theta)
$$


que establece el valor

$$
R=\kappa_{0} \tan (\theta)
$$

A su vez, observando la misma figura, podemos plantear

$$
\begin{gathered}
\kappa_{0}^{2}+R^{2}=L^{2} \\
\kappa_{0}^{2}+\kappa_{0}^{2} \tan ^{2}(\theta)=r^{2} \cos ^{2}(\phi-\varphi)
\end{gathered}
$$

con lo cual

$$
\kappa_{0}=\frac{r \cos (\phi-\varphi)}{\sqrt{1+\tan ^{2}(\theta)}}
$$

Con las expresiones obtenidas (B.6, B.7 y B.9) se calcula la distancia

$$
r_{0}=\sqrt{\left(x_{i}-x_{0}\right)^{2}+\left(y_{i}-y_{0}\right)^{2}+\kappa_{0}^{2}}
$$

que representa la distancia mínima entre el punto de llegada de la partícula al suelo y el eje de la lluvia. 


\section{Apéndice $\mathrm{C}$}

\section{Parámetros $\beta$}

Se presentan a continuación los valores del parámetro $\beta$ obtenidos en el ajuste de la distribución lateral para lluvias simuladas sobre diferentes perfiles atmosféricos desarrollados por el modelo GAMMA. En la tabla estos perfiles están identificados por el valor de la temperatura en el suelo. La función utilizada en el ajuste es la utilizada oficialmente en el Observatorio Auger 8.1.

\begin{tabular}{|c||c|c|c|}
\hline \hline Atmósfera & $\mathbf{1 . 0}^{17} \mathbf{e V}$ & $\mathbf{1 . 0}^{18} \mathbf{e V}$ & $\mathbf{1 . 0}^{19} \mathbf{e V}$ \\
\hline 6 & $-1.915 \pm 0.005$ & $-1.928 \pm 0.017$ & $-2.091 \pm 0.023$ \\
\hline 8 & $-1.949 \pm 0.023$ & $-1.901 \pm 0.016$ & $-2.129 \pm 0.026$ \\
\hline 10 & $-1.969 \pm 0.016$ & $-1.941 \pm 0.016$ & $-2.119 \pm 0.034$ \\
\hline 12 & $-1.934 \pm 0.013$ & $-1.892 \pm 0.024$ & $-2.209 \pm 0.016$ \\
\hline 14 & $-1.961 \pm 0.018$ & $-1.871 \pm 0.011$ & $-2.036 \pm 0.021$ \\
\hline 16 & $-1.981 \pm 0.013$ & $-1.921 \pm 0.018$ & $-2.040 \pm 0.021$ \\
\hline 20 & $-1.925 \pm 0.012$ & $-1.913 \pm 0.012$ & $-2.063 \pm 0.012$ \\
\hline 22 & $-1.976 \pm 0.025$ & $-1.953 \pm 0.016$ & $-2.106 \pm 0.019$ \\
\hline \hline
\end{tabular}




\begin{tabular}{|c||c|c|c|}
\hline \hline Atmósfera & $\mathbf{1 . 0}^{17} \mathbf{e V}$ & $\mathbf{1 . 0}^{18} \mathbf{e V}$ & $\mathbf{1 . 0}^{19} \mathbf{e V}$ \\
\hline 24 & $-1.987 \pm 0.020$ & $-2.046 \pm 0.017$ & $-2.005 \pm 0.019$ \\
\hline 26 & $-1.999 \pm 0.019$ & $-1.931 \pm 0.017$ & $-2.165 \pm 0.019$ \\
\hline 28 & $-1.909 \pm 0.023$ & $-1.905 \pm 0.017$ & $-2.035 \pm 0.034$ \\
\hline 30 & $-1.936 \pm 0.009$ & $-1.862 \pm 0.015$ & $-2.018 \pm 0.023$ \\
\hline 32 & $-1.900 \pm 0.015$ & $-1.910 \pm 0.029$ & $-2.044 \pm 0.029$ \\
\hline 34 & $-1.955 \pm 0.039$ & $-1.909 \pm 0.014$ & $-2.016 \pm 0.018$ \\
\hline \hline
\end{tabular}




\section{Bibliografía}

[1] M. Nagano, A. A. Watson, Review of Modern Physics Vol 72, No 3 (2000).

[2] P.Auger, R. Maze, T Grivet-Mayer, Academie des Sciences, París 206, $1721(1938)$

[3] P. Auger, P. Ehrenfest Jr., R. Mazze, J. Daudin, C. Robley, A. Freón, Rev. Mod. Phys. 11, 288 (1939)

[4] G. W. Clark, J. Earl, W. L. Kraushaar, J. Linsley, B. B. Rossi, F. Scherb, D. Scott, Phys. Rev. Lett. 122, 637 (1961)

[5] Y. Toyoda et al. en Proceedings of the 9th International Cosmic Ray Conference, London (Institute of Physics, London) Vol. 2, p. 708 (1965)

[6] A.A. Penzias, R. W. Wilson, Astrophysics J. 142, 419 (1965)

[7] K. Suga en Proceedings of 5th Interamerican Seminar on Cosmic Rays, editado por I. Escobar et al. (Laboratorio de Física cósmica de la Universidad Mayor de San Andrés, La Paz, Bolivia), Vol. 2, p. XLIX (1962)

[8] A. E. Chudakov en Proceedings of 5th Interamerican Seminar on Cosmic Rays, editado por I. Escobar et al. (Laboratorio de Física cósmica de la Universidad Mayor de San Andrés, La Paz, Bolivia), Vol. 2, p. XLIX (1962)

[9] T. Hara, S. Kawaguchi, S. Mikamo, M. Nagano, K. Suga, G. Tanahashi, K. Uchino, H. Akiyama, Acta Phys. Acad. Sci. Hung. 29, Suppl 3, 361 (1970).

[10] H. E. Bergeson, G. L. Cassiday, T. W. Chiu, D. A. Cooper, J. W. Elbert, E. C. Loh, D. Steck, W. J. West, J. Boone, J. Linsley, Phys. Rev. Lett. 39, 847, (1977).

[11] V. S. Berezinsky, Nucl. Phys. B (Proc. Suppl.) 70 ,419 (1999) 
[12] P. L. Biermann, J. Phys. G 23, 1 (1997)

[13] P. Bhattacharjee, G. Sigl, Phys. Rep. 327, 110 (2000).

[14] A. M. Hillas, Nature (London) 395, 15 (1998).

[15] A. M. Hillas, Nucl. Phys. B (proc. Suppl.) 75 B, 109 (1999)

[16] E. Fermi, Phys. Rev.75, 1169 (1949).

[17] R. J. Protheroe, en Proceedings of the 10th course of International School of Cosmic Ray Astrophysics Erice, Italia. pre-print astroph/9612212 (1997).

[18] L. O. C. Drury, Rep Prog. Phys.46, 973 (1983).

[19] J. J. Jokipii, Ap. J. 313, 842 (1987)

[20] L. O. C. Drury, Contemp, Phys. 35, 231 (1994)

[21] P. A. Shaver, M. Pierre, Astron. Astrophys. 220, 35 (1989)

[22] M. T. Dova, Nuc. Physics B (Proc. Suppl) 122, 170-177 (2003).

[23] K. Greissen, Phys. Rev. Lett., 16, 748, (1966).

[24] Z. T. Zatsepin, V. A. Kuz'min, Zh Eksp. Teor. Fiz Pis'ma REed. 4, 144 (1966).

[25] A. M. Hillas, Can. J. Phys. 46, S623 (1968).

[26] C. T. Hill, D. N. Scramm, T. P. Walker, Phys. Rev. D, 36, 1007 (1987).

[27] V. S. Berezinsky, S. I. Grigor'eva Astron. Astrophys. 199, 1 (1988).

[28] F. W. Stecker, Nature (London) 324,401 (1989).

[29] J. P. Rachen, P. L. Biermann, Astron. Astrophys 272, 161 (1993).

[30] S. Yoshida, M. Teshima, Prog. Theor. Phys. 89, 833 (1993).

[31] R. J. Protheroe, P. Johnson, Astropart. Phys.4, 253 (1996).

[32] L. A. Anchordoqui, M. T. Dova, L. N. Epele, J. Swain, Phys. Rev. D, 55, 7536 (1997).

[33] L. A. Anchordoqui, M. T. Dova, L. N. Epele, J. Swain, Phys. Rev. D, 57, 7103 (1998). 
[34] J. L. Puget, F. W. Stecker, J. H. Bredekamp, Astrophys. J. 205638 (1976).

[35] G. R. Blumenthal, Phys. Rev. D 1, 1596 (1970).

[36] M. A. Malkan, F. W. Stecker, Astrophys J. 496, 13 (1998).

[37] L. N. Epele, E. Roulet, Phys. Rev. Lett. 81, 3295 (1998).

[38] F. W. Stecker, M. H. Salomon, Astrophys J. 512, 521 (1999).

[39] Particle Data Group, Particle Data Book (2004).

[40] S. Eidelman et al. [Particle Data Group collaboration], Phys. Lett. B 592, 1 (2004).

[41] T. J. L. McComb, R. J. Protheroe, K. E. Turver, J. Phys. G 5, 1613 (1979).

[42] L. A. Anchordoqui, M. T. Dova, A. Mariazzi, T. McCauley, T. Paul, S. Reucroft, J. Swain, arXiv:hep-ph/0407020 v2 (2004).

[43] A. A. Watson, Nucl. Phys. B 28B, 3 (1992).

[44] J. Linsley, Phys Rev. Letter 10, 146 (1963).

[45] P. Privitera for the Auger Collaboration, en The 28th International Cosmic Ray Conference Tsukuba, Japón, Publicado en "Tsukuba 2003, Cosmic ray" 357-360 (2003).

[46] A. M. Hillas, Acta Phys. Sci. Hung., 29 Suppl. 3, 355 (1970)

[47] K. Meisenheimer et al., Astron. Astrophys. 219, 63 (1989).

[48] P. Billoir, P. Da Silva. Auger Technical Note, GAP 2002-073 (2002).

[49] M Roth for the Auger Collaboration, en The 28th International Cosmic Ray Conference Tsukuba (Japón). Publicado en "Tsukuba 2003, Cosmic Ray" 333-336, arXiv:astro-ph/0308392 v1 (2003).

[50] R. N. Coy et al. Astrop. Phys. 6, 263 (1997).

[51] W. Heitler The Quantum Theory of Radiation, 2nd Edition (Oxford University Press, London, 1944).

[52] J.Linsley, A. A. Watson, Phys. Review Letteres 46, 459 (1981) 
[53] Pierre Auger Collaboration (J. Abraham et al.). 46pp. Nucl. Instrum. Meth.A523:50-95, (2004).

[54] The Pierre Auger Observatory Design Report (1998).

[55] P. Bauleo, A. Castellina, R. Knapik, G. Navarra, J. Harton, Auger Technical Note, GAP-2004-047 (2004)

[56] R. Brun and F. Rademakers, ROOT - An Object Oriented Data Analysis Framework, Proceedings AIHENP'96 Workshop, Lausanne, Sep. 1996, Nucl. Inst. Meth. in Phys. Res. A 389 (1997) 81-86. See also http://root.cern.ch

[57] J. Bluemer, R. Engel, J.R. Hoerandel. Prog.Part.Nucl.Phys.63:293338,2009 .

[58] The Pierre Auger Collaboration, Physics Letters B685 (2010) 239.

[59] V. Berezinsky, Proceedings of the 30th International Cosmic Ray Conference, Merida (Mexico) (2007), arXiv:0710.2750.

[60] T. Stanev, R. Engel, A. Mucke, R. Protheroe, and J. Rachen, Phys. Rev. D62, 093005 (2000), astro-ph/0003484.

[61] D. De Marco and T. Stanev, Phys. Rev. D72, 081301 (2005).

[62] T. Wibig and A. W. Wolfendale, J. Phys. G31, 255 (2005).

[63] D. Allard, E. Parizot, and A. Olinto, Astropart. Phys. 27, 61 (2007), astro-ph/0512345.

[64] D. Allard, A. Olinto, and E. Parizot, Astron. Astrophys. (2007), astroph/0703633.

[65] V. Berezinsky, S. Grigoreva, and B. Hnatyk, Nucl. Phys. (Proc. Suppl.) 151, 497 (2006).

[66] M. Tueros, Tesis doctoral (2010)

[67] The Pierre Auger Collaboration, Science 318, 939 (2007).

[68] V. Ginzburg and S.I.Syrovatskii, Pergamon Press, Russian edition (1963).

[69] P. L. Biermann and P. A. Strittmatter, Astrophys. J. 322, 643 (1987). 
[70] R. Protheroe and A. Szabo, Phys. Rev. Lett. 69, 2885 (1992).

[71] G. Benford and R. Protheroe, arXiv:0706.4419 [astro-ph].

[72] The Pierre Auger Collaboration, Phys. Rev. Letters 104 (2010) 091101.

[73] X. Bertou et al. [Pierre Auger Collaboration], Nucl. Instrum. Meth., A568, p. 839 (2006).

[74] P. Ghia, Auger Technical Note, GAP-2004-018.

[75] P. L. Ghia and G. Navarra, Auger Technical Note, GAP-2003-007.

[76] E. Parizot, I. Lhenry, D. Allard, P. Ghia, and G. Navarra, Auger Technical Note, GAP-2004-023.

[77] R. Sato, J. Bellido, H. Reis and C. Escobar, Auger Technical Note, GAP-99-014.

[78] G. Borreani et al. Nucl. Instrum. Meth., A461, p. 577 (2001).

[79] R. Knapik et al. [Pierre Auger Collaboration] 2007 Proc. 30th ICRC (Merida), arXiv:0708.1924

[80] A.Schmidt, T.Asch, M.Kleifges, H.-J.Mathes, and H.Gemmeke, Auger Technical Note, GAP-2007-118.

[81] S.Y. BenZvi et al. Nucl. Instr. and Meth. A, 574, p. 171184, (2007).

[82] The Pierre Auger Collaboration, Nucl. Instrum. Meth., A523, p. 50 (2004)

[83] B. Fick et al. JINST, 1, P11003, 2006.

[84] A. Anzalone, F. Isgro, and D. Tegolo, en Proceedings of the 2008 CRIS, Malfa.

[85] T. Huege, for the Pierre Auger Collaboration, Nucl. Instrum. Meth. A617, p.484-487, (2010).

[86] H. O. Klages for the Pierre Auger Collaboration, en Proceedings of the 30th International Cosmic Ray Conference (2007). FZKA-7340 pp 101-104.

[87] M. Platino, en Proceedings of the 31th International Cosmic Ray Conference (2009). 
[88] R. Cester for the Pierre Auger Collaboration, en Proceedings of the 29th International Cosmic Ray Conference, Pune (India), 8, p. 347.

[89] A. Remoto, Auger Technical Note, GAP-2009-127.

[90] W. Weischet, Einfuhrung in die Allgemeine Klimatologie, B. G. Teubner (1977). Obtenido de [93].

[91] Leitf den fur die Ausbildung im Deutschen Wetterdienst. Allgemeine Meteaorologie. Selbstverlag des Deutschen Wetterdienstes, Offenbach am Main, 3. Auflage, 1987. Obtenido de [93].

[92] Tomado del curso Astr201 de C. Mihos, Department of Astronomy, Case Western Reserve University (www.cwru.edu). Disponible en el sitio web del curso http://burro.cwru.edu/Academics/Astr201 .

[93] M. Will, Auger Technical Note, GAP-2009-081.

[94] No se encuentran referencias oficiales acerca del modelo de Linsley. Las referencias: A. M. Hillas, Nucl. Phys. B (Proc. Suppl.), 52B, 29 (1997); y A. M. Hillas, en Proceedings of the 19th International Cosmic Ray Conference, La Jolla (USA), 1, 155 (1985), contienen información acerca de la parametrización.

[95] B. Keilhauer, B. Wilczynska y H. Wilczynski, Auger Technical Note, GAP-2005-021 (2005).

[96] B. Keilhauer, R. Engel, B. Wilczynska y H. Wilczynski and M. Will, Auger Technical Note, GAP-2009-037 (2009).

[97] B. Wilczynska , D. Gra1 , P. Homola , B. Keilhauer , H. Klages , J. Pkala and H. Wilczynski, en Proceedings of the 28th International Cosmic Ray Conference Tsukuba (Japón).

[98] Ver http://badc.nerc.ac.uk/data/radiosglobe/radhelp.html

[99] National Aeronautics and Space Administracion (NASA). USStandard Atmosphere 1976, NASA-TM-X-74335 (1976) http://modelweb.gsfc.nasa.gov/atmos/us_standard.html.

[100] S. Sciutto, Proceedings of the 27th International Cosmic Ray Conference, Hamburgo (ALemania), 1, 237 (2001);

[101] S. Sciutto, AIRES User's Manual and Reference Guide version 2.6.0 (2002) disponible en www.fisica.unlp.edu.ar/auger/aires. 
[102] S. J. Sciutto, Auger Technical Note, GAP-98-032 (1998).

[103] J.C. Moreno, S. Sciutto, Characterization of the Atmospheric Depth Profile using the ground level Temperature. Aceptado para su publicacin por el Comit de Publicaciones del Obserrvatorio Pierre Auger. Enviado a Astroparticle Physics.

[104] J. C. Moreno, S. Sciutto y M. Tueros, Auger Technical Note, GAP 2007-082.

[105] J. C. Moreno, Atmospheric Depth Profile: A new parameterization using Temperature at ground, Presentado en Auger Collaboration Meeting. Malargüe 22 al 29 de Abril de 2007.

[106] J. C. Moreno, Atmospheric Depth Profile: A new model to an old subject, Presentado en International School of Cosmic Ray Astrophysics, 16th Course: "Gamma Ray and Cosmic Ray Astrophysics: From below $\mathrm{GeV}$ to beyond EeV Energies". Erice (Italia) 5 al 12 de Julio de 2008.

[107] Graw Radiosondes GmbH \& Co. KG. (www.graw.de).

[108] B. A. Bodhaine et al., J. Atmos. Ocean. Tech., Vol. 16, 1854 (1999), basado en List, R. J., 1968: Smithsonian Meteorological Tables. Smithsonian, $527 \mathrm{pp}$.

[109] D. Sonntag, Important New Values of the Physical Constants of 1986, Vapour Pressure Formulations based on the IST-90 and Psychrometer Formulae; Z. Meteorol., 70 (5), pp. 340-344, 1990. Los parámetros de la fórmula fueron extraídos de la referencia [93].

[110] J. C. Moreno, Atmospheric Depth Profile: Update in the new model, Presentado en Auger Collaboration Meeting. Malarge 12 al 17 de Noviembre de 2007.

[111] J. C. Moreno, Update in the study of the Atmospheric Depth Profile, Presentado en Auger Collaboration Meeting. Malargüe 6 al 12 de Marzo de 2010.

[112] J. C. Moreno, New Improvements in the Atmospheric Depth Profile, Presentado en Auger Collaboration Meeting. Malargüe 14 al 20 de Noviembre de 2009 . 
[113] Ver http://weather.uwyo.edu/upperair/sounding.html.

[114] D. Barnhill, P. Bauleo, M. T. Dova, J. Harton, R. Knapik, J. Knapp, J. Lee, M. Manceñido, A. G. Mariazzi, I. C. Maris, D. Newton, M. Roth, T. Schmidt and A. Watson, 2005 Proc. 29th ICRC (Pune), 00, p. 101-106.

[115] T. Schmidt, I. C. Maris and M. Roth, Auger Technical Note, GAP 2007-016.

[116] Software provisto por el Dr. S. Sciutto.

[117] B.Keilhauer, J. Blümer, R. Engel, H.O. Klages and M. Risse, Astropart. Phys. 22 (2004) 249-261.

[118] D. Heck and T. Pierog, CORSIKA User's Guide version CORSIKA 6.7 (2007), disponible en wwwik.fzk.de/corsika/usersguide/corsika_tech.html.

[119] N. N. Kalmykov, S. S. Ostapchenko, A. I. Pavlov, Nucl. Phys. B (Proc. Suppl.) 52B, 17, (1997).

[120] B. Keilhauer, H. Blümer, H. O. Klages and M. Risse, Auger Technical Note, GAP 2002-022.

[121] D. Veberic and M. Roth - Offline team, Offline Reference Manual - SD Reconstruction, Auger Technical Note, GAP 2005-035.

[122] Pierre Auger Collaboration, Astropart.Phys. 35 (2012) 591-607. 


\section{Agradecimientos}

Esta Tesis representa el trabajo realizado en varios años, lo que implica una serie de agradecimientos muy importante. En particular quisiera agradecer a mi director Sergio Sciutto por su dirección y acompañamiento en este proceso. Sin dudas a Analisa y Matías debo agradecerles enormemente por las fructíferas interacciones que desembocaron en grandes aportes. A Carlos García Canal por su apoyo y conducción del grupo. Quiero agradecer también a personas que en diferentes momentos forman o formaron el equipo del Grupo Auger La Plata: Teresa, Flavia, Patricia, Daniel, Mónica, Hernán, Tatiana y Luis.

Agradezco el apoyo del Departamento de Física, la Facultad de Ciencias Exactas y el CONICET. No sólo a las instituciones, sino también a todas las personas detrás de ellas.

Al grupo de la Universitá Tor Vergata, Gonzalo, Claudio, Giorgio y Valerio; que tan amablemente me permitió vivir la grata experiencia del trabajo en una Universidad extranjera.

A todos los colegas y amigos que conocí durante los meetings y las estancias de shift en Malargüe, no sólo a los físicos e ingenieros, sino a todo el personal del Observatorio, excelentes personas y profesionales. Sería injusto destacar sólo algunos.

Pero más allá de las personas estrictamente vinculadas al proceso de investigación de la Tesis, están aquellos que me bancaron de alguna u otra manera para este trabajo. Dentro de esta gran lista empiezo agradeciendo a la gente del Taller de Enseñanza de Física. No los enumero por temor a olvidar alguno. Bah... empiezo con algunos: Osvaldo , Román, Lean, Santi G., Pía, pero el agradecimiento es para todos. Entiendan que son una banda enorme!

A los amigos de la facultad: Leticia, Santiago, Tito, Carlos, Pablo P, Pablo RP, Nico, Raulo y demás; porque esos momentos de compañía también son parte del proceso. 
Aprovecho la oportunidad, probablemente la última de un documento escrito en un formato símil libro, para agradecer a otra gente muy importante en mi vida. No concibo el haber podido realizar este camino sin el afecto de todo un grupo de gente extra-universidad (que fueron el soporte más importante) y que estuvieron siempre conmigo en todos estos años. Mis padres, hermanos y hermanas, cuñados y cuñadas, sobrinos y sobrinas, primo y tías (no los enumero por la cantidad de Morenos, Elizondos y afines que somos).

Por último cierro con quien abrí este documento: a Ceci. Como escribí años antes en la tesina: ésto también es parte de nuestra construcción. 